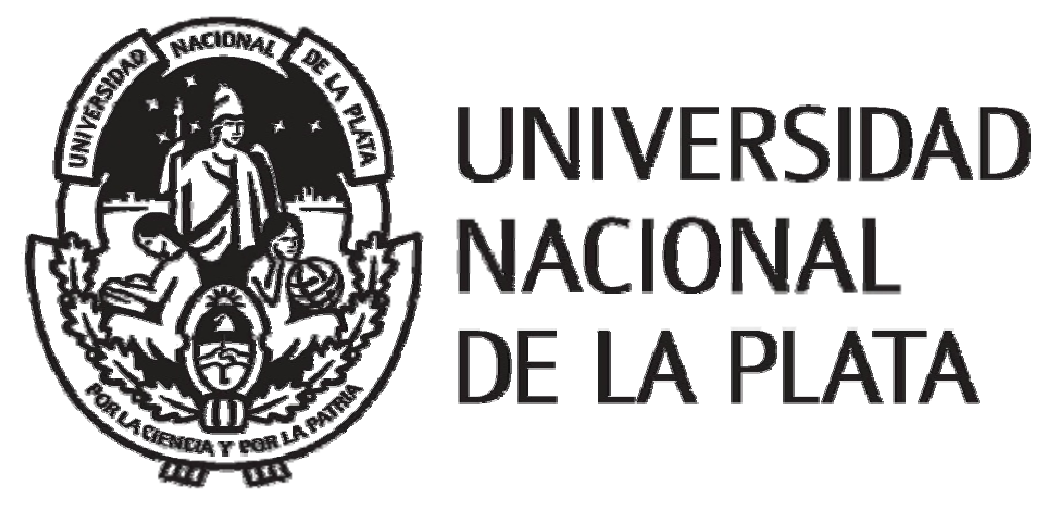

\title{
Mitigación de campos magnéticos originados por instalaciones de transmisión y distribución de energía eléctrica
}

Tesis de Maestría

\section{Ing. Carlos Alberto Wall}

Presentada ante la Facultad de Ingeniería de la Universidad Nacional de La Plata como requisito para la obtención del grado académico de

\section{MAGISTER EN INGENIERIA}

Dirección de tesis:

Director: Prof. Ing. Patricia Liliana Arnera

Codirector: Prof. Ing. María Beatriz Barbieri

Jurado de tesis:

Dr. Marcelo Trivi

Dr. Ing. Walter Giménez

Dr. Ing. Ricardo Diaz 
A mis padres Alberto y Gladis, a mi esposa Vanesa y a mis hijas Sofía y Olivia 


\section{Agradecimientos}

Agradezco a mis directores de tesis, Patricia y Beatriz, por el tiempo, el esfuerzo, la motivación y los conocimientos transmitidos.

También a los compañeros de trabajo y amigos del IITREE-LAT por la colaboración prestada en todo momento.

La realización de este trabajo fue posible gracias al Instituto de Investigaciones Tecnológicas para Redes y Equipos Eléctricos (IITREE-LAT). 


\section{Contenido}

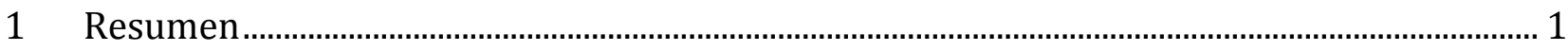

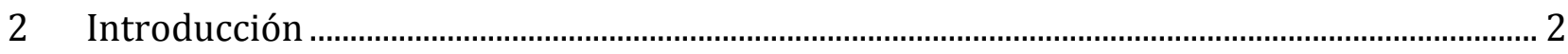

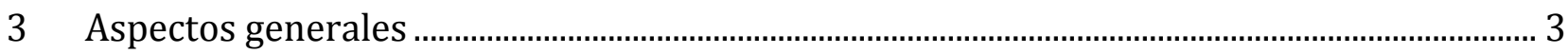

3.1 Fuentes de Campo Magnético ...................................................................................... 3

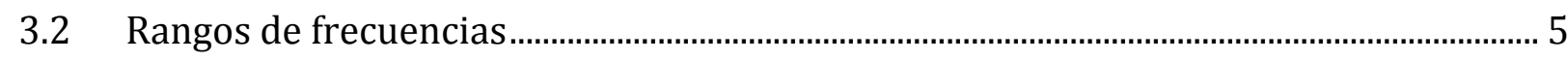

3.3 Definiciones ......................................................................................................................... 5

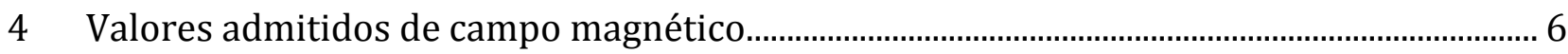

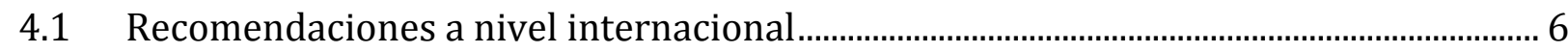

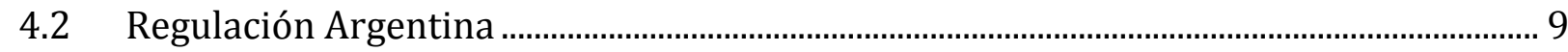

4.3 Regulaciones adoptadas por diferentes países ................................................................. 10

5 Campos magnéticos en sistemas de transmisión y distribución de energía eléctrica ......11

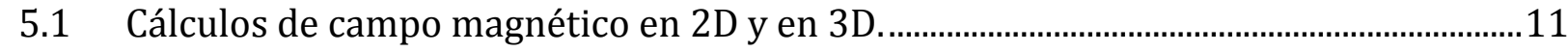

5.2 Campo Magnético generado por Líneas Aéreas...................................................................12

5.3 Campo Magnético generado por Cables Subterráneos........................................................13

5.3.1 Campo Magnético generado por cables subterráneos simple terna........................14

5.3.2 Campo Magnético generado por cables subterráneos con múltiples ternas .......16

5.4 Campo Magnético generado por Estaciones Transformadoras .........................................17

5.5 Campo Magnético generado por Centros de Transformación............................................18



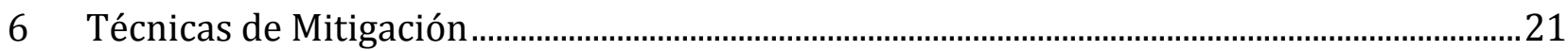

6.1 Incremento de la distancia a la fuente de campo magnético ...........................................21

6.2 Ubicación de conductores....................................................................................................21

6.3 Cambio de secuencia de energización ................................................................................23

6.3.1 Ejemplos de cambios de secuencias de energización en LAAT -Doble Terna.....23

6.3.2 Ejemplos de cambios de secuencias de energización en CS -Doble Terna ...........27

6.3.3 Análisis de los resultados ............................................................................................. 30

6.4 Mitigación utilizando materiales metálicos ........................................................................31

6.4.1 Experiencias en laboratorio....................................................................................... 33

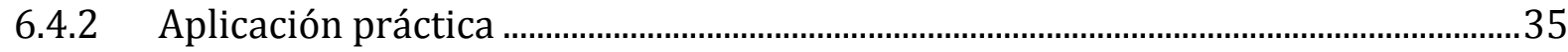

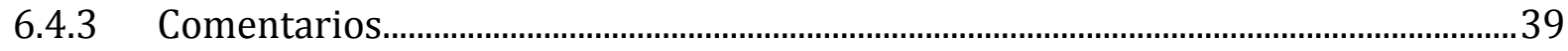

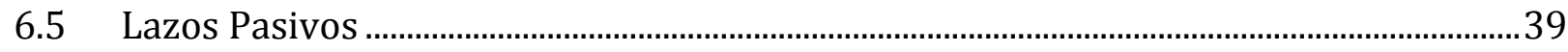

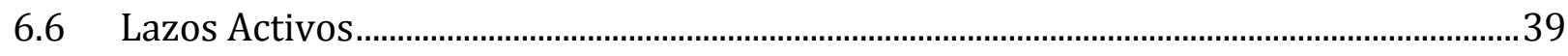

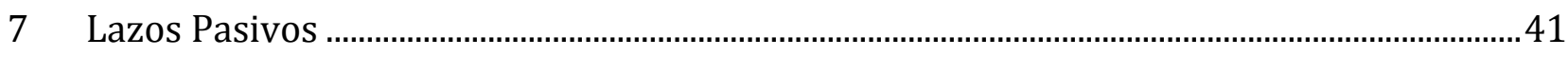

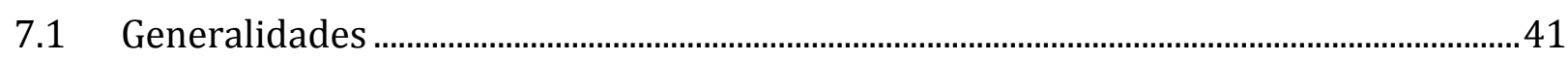

7.1.1 Hipótesis para el cálculo de la corriente inducida en un lazo.................................... 41 
7.1.2 Principales características de un lazo pasivo...............................................................42

7.1.3 Inductancia mutua entre un conductor de fase y un Lazo "LM $\mathrm{CL}_{\mathrm{CL}}$ "........................... 44

7.1.4 Reactancia inductiva conductor - lazo "X $\mathrm{XLCL}_{\mathrm{LMC}}$ ”................................................................ 44

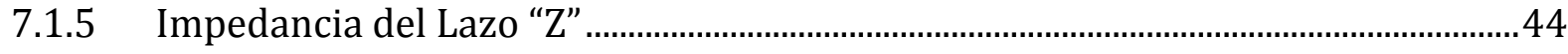

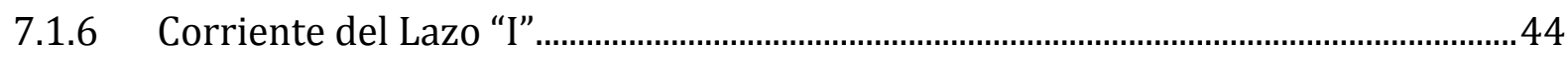

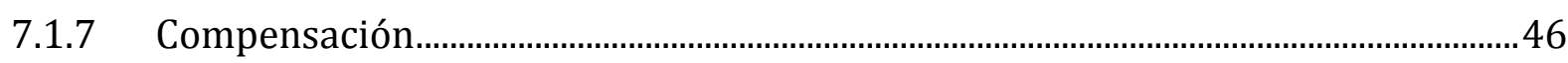

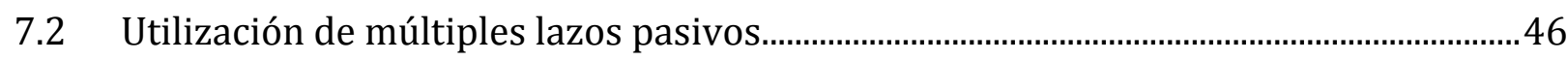

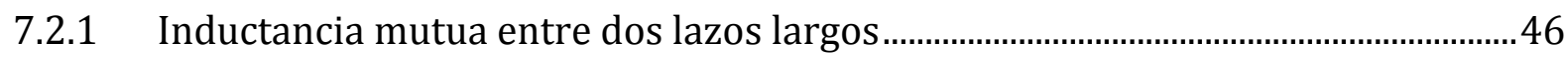

7.2.2 Inductancia mutua entre dos lazos cortos..................................................................

7.2.3 Corriente Inducida en los lazos - Utilización de múltiples lazos.................................47

7.3 Desarrollo de herramientas de cálculo ...................................................................................... 49

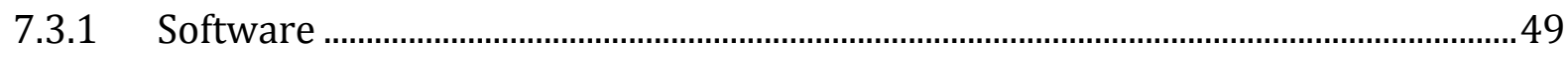

7.4 Implementación práctica y Validación de modelos.............................................................54

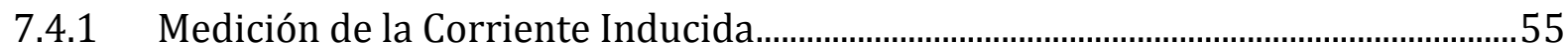

7.4.2 Análisis de la Impedancia de Lazos Pasivos .................................................................57

7.4.3 Resultados Obtenidos con cálculo y medición de campo magnético - Lazo único 58

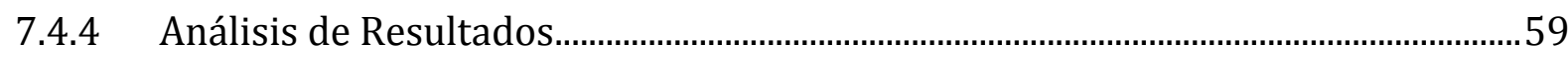

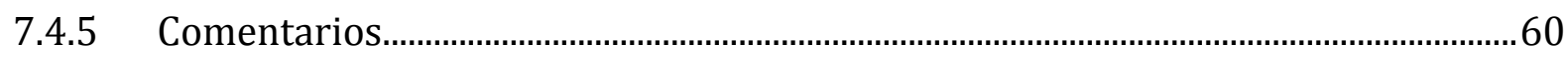

7.4.6 Resultados obtenidos con cálculos y mediciones- Más de un lazo ...........................61

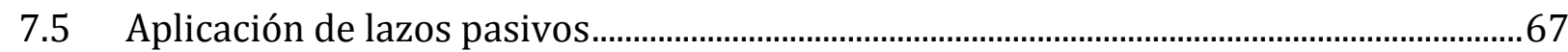

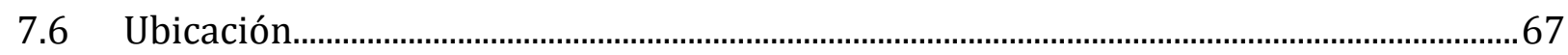

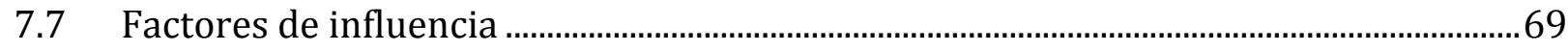

7.7.1 Número de lazos pasivos empleados............................................................................... 71

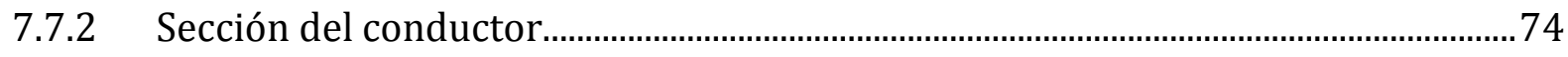

7.7.3 Configuración de los lazos pasivos ...............................................................................

7.7.4 Distancia entre Lazos y conductores de fase..............................................................77

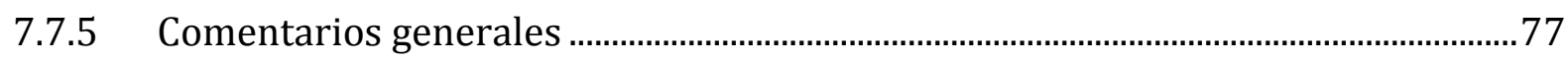

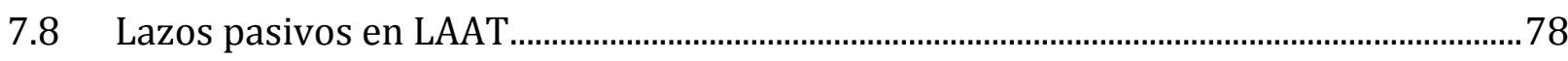

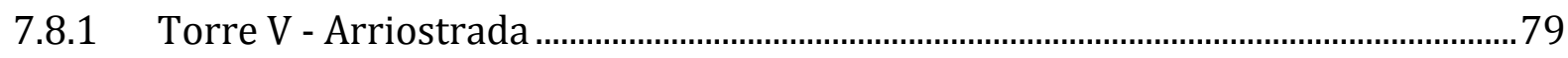

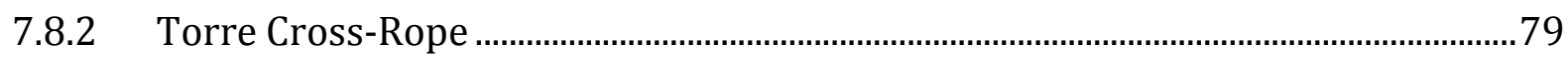

8 Ejemplo de aplicación de la herramienta de cálculo desarrollada..........................................81

9 Consideraciones finales ........................................................................................................... 86

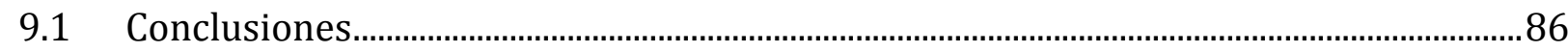

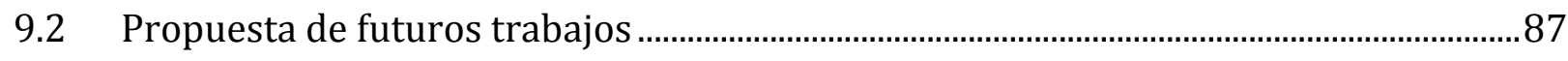

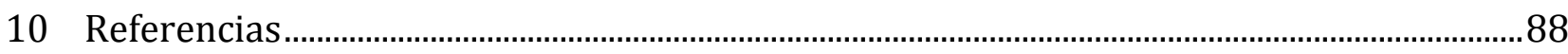




\section{Anexos}

\section{Anexo I}

Mecanismos de acoplamiento entre el campo magnético y el cuerpo

Análisis de la IARC

Definiciones - ICNIRP 2010

Fundamentación de las líneas de orientación - ICNIRP 2010

Directrices para limitar la exposición a los CEM

Limitaciones básicas y niveles de referencia

Niveles de referencia de las principales guías a nivel internacional

\section{Anexo II}

Ecuaciones de Maxwell

Campo Magnético generado por un conductor rectilíneo e infinito

Campo Magnético debido a una Línea

Campo Magnético generado por una línea trifásica

Influencia de la tierra en el Campo Magnético generado por una Línea Aérea

Campo Magnético generado por una línea de transmisión con corrientes equilibradas

Campo Magnético generado por una línea de transmisión en general

Resistencia y Reactancia inductiva de circuitos con retorno por el suelo

\section{Anexo III}

Polarización del campo magnético

Ejemplos de polarización del campo magnético

\section{Anexo IV}

Descripción del software comercial - Opera 3D ELEKTRA SS

Condiciones de borde

Condiciones de borde en caso de simetría

\section{Anexo V}

Datos geométricos de LAAT y CSAT

\section{Anexo VI}

Medición de campo magnético

Calibración de medidores de campo magnético

\section{Anexo VII}

Análisis de sensibilidad a la disposición de conductores - Simple Terna

Análisis de sensibilidad a diferentes secuencias de energización - Doble Terna

\section{Anexo VIII}

Condiciones de borde utilizadas en mitigación con placas metálicas 


\section{Anexo IX}

Configuraciones de lazos pasivos consideradas para CSAT

Perfiles de campo magnético y FA obtenidos para el Caso 1

Perfiles de campo magnético y FA obtenidos para el Caso 2

Perfiles de campo magnético y FA obtenidos para el Caso 3

Perfiles de campo magnético y FA obtenidos para el Caso 4

Perfiles de campo magnético y FA obtenidos para el Caso 5 


\section{Lista de Figuras}

Fig. 1 - Espectro electromagnético 4

Fig. 2 - Configuraciones de LAAT 2x220kV ............................................................................... 12

Fig. 3 - Valores de Campo Magnético, generados por LAAT 2x220kV (I=1500A)...................... 13

Fig. 4 - Ejemplo de disposición de conductores en zona de ductos y fosa de empalmes, CSAT $220 \mathrm{kV}$.

Fig. 5 - Fosa de empalmes, CSAT $220 \mathrm{kV}$.

Fig. 6 - Campo magnético generado por CSAT $220 \mathrm{kV}$, en zona de ductos y fosa de empalmes.15

Fig. 7 - Perfiles de campo magnético generado por CSAT $220 \mathrm{kV}$, en zona de ductos y fosa de empalmes.

Fig. 8 - Ejemplo de disposición de conductores en zona de ductos y fosa de empalmes, CSAT $2 \times 132 \mathrm{kV}$.

Fig. 9 - Mapa de niveles de B, CSAT doble terna................................................................................. 16

Fig. 10 - Perfiles Transversales, CSAT doble terna. ......................................................................... 16

Fig. 11 - Campo magnético generado por una subestación transformadora. ................................ 17

Fig. 12 - Layout del CT considerado. ............................................................................................ 18

Fig. 13 - Mapas de B, en un CT.

Fig. 14 - Curvas de nivel de B, en un CT. .................................................................................... 19

Fig. 15 - Valores de campo B medidos y calculados, LAAT 2 x $220 \mathrm{kV} \mathrm{CH-AS,} \mathrm{I=200} \mathrm{A.............} 19$

Fig. 16 - Valores de campo B medidos y calculados, LAAT 2 x $220 \mathrm{kV} \mathrm{CV-AS,} \mathrm{I=1300} \mathrm{A...........} 20$

Fig. 17 - Valores de campo B medidos y calculados, LAAT 2 x $220 \mathrm{kV} \mathrm{CV-LP,} \mathrm{I=650} \mathrm{A............} 20$

Fig. 18 - Valores de campo B $[\mu \mathrm{T}]$, para diferentes distancias a la fuente, $\mathrm{I}=2000 \mathrm{~A}$.................. 21

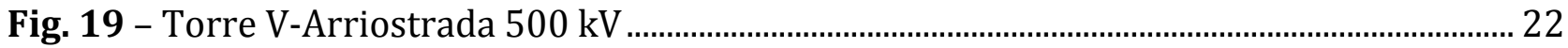

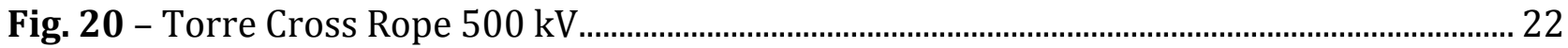

Fig. 21 - Valores de campo magnético en LAAT 500 kV VA, I=1500 A.......................................... 23

Fig. 22 - Valores de campo magnético en LAAT 500 kV CR, I=1500 A.......................................... 23

Fig. 23 - Valores de campo B calculados, LAAT 2 x $220 \mathrm{kV}$ coplanar horizontal (fase R: verde, fase S: Azul y fase T: rojo), I=1500 A................................................................................... 24

Fig. 24 - Valores de campo B y FA, LAAT 2 x $220 \mathrm{kV}$ coplanar horizontal, I=1500 A................. 24

Fig. 25 - Valores de campo B calculados, LAAT 2 x $220 \mathrm{kV}$ coplanar vertical (fase R: verde,

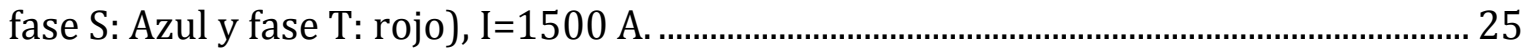

Fig. 26 - Valores de campo B y FA, LAAT 2 x $220 \mathrm{kV}$ coplanar vertical, I=1500 A.................... 25

Fig. 27 - Valores de campo B calculados, LAAT 2 x $220 \mathrm{kV}$ Coplanar vertical (fase R: verde, fase S: Azul y fase T: rojo), I=1500 A. ……...................................................................... 26

Fig. 28 - Valores de campo B y FA, LAAT 2 x 220 kV coplanar horizontal, I=1500 A................ 27

Fig. 29 - Mapa de niveles de B, Caso 2 ............................................................................................... 28

Fig. 30 - Perfiles Transversales, Caso 2 .......................................................................................... 28

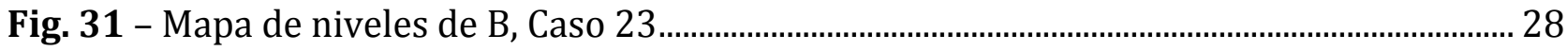

Fig. 32 - Perfiles Transversales, Caso 3 ……................................................................................... 28

Fig. 33 - Mapa de niveles de B, Caso 4 ................................................................................................ 29

Fig. 34 - Perfiles Transversales, Caso 4 ................................................................................................... 29



Fig. 36 - Perfiles Transversales, Caso 5 .......................................................................................... 29

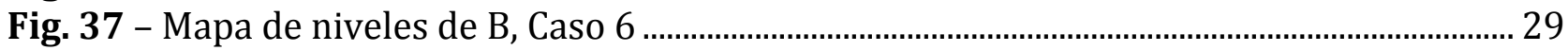

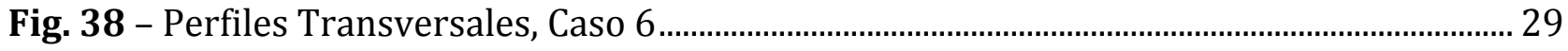

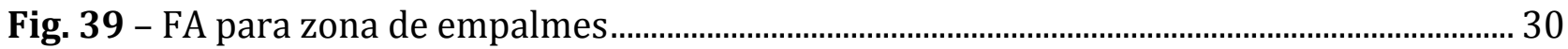

Fig. 40 - FA para zona de ductos .............................................................................................................. 30

Fig. 41 - Alternativas de apantallamiento de Campo Magnético, utilizando láminas

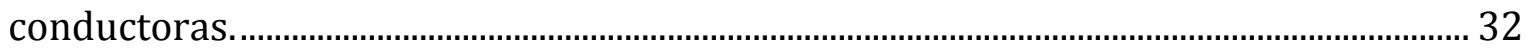


Fig. 42 - Modelo experimental de espira y placa de blindaje. …….................................................. 33

Fig. 43 - Modelo de simulación de espira (Rojo) y placa de blindaje (Azul)................................. 33

Fig. 44 - Campo magnético simulado considerando placas de distintos espesores................... 34

Fig. 45 - FA considerando valores simulados, considerando placas de distintos espesores... 34

Fig. 46 - Campo magnético medido y simulado (Aire, $\mathrm{Fe}, \mathrm{Al}$ ), con diferentes placas.................. 34

Fig. 47 - FA considerando valores simulados (Aire, Fe, Al), considerando diferentes placas. 34

Fig. 48 - Densidad de corriente en placa cuyas características son $\sigma=8,410^{6} \mathrm{~S} / \mathrm{m}, \mu_{\mathrm{r}}=240$ y espesor igual a $2,5 \mathrm{~mm}$.

Fig. 49 - Vista superior de la geometría del puesto de transición, conductores (Rojo), estructura metálica (Verde) y protección mecánica (Azul). .................................................. 36

Fig. 50 - Modelo que incluye los conductores de bajada, la protección mecánica y la torre.... 36

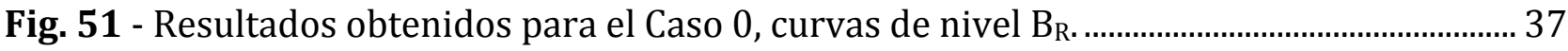

Fig. 52 - Resultados obtenidos para el Caso 1, curvas nivel $B_{R}$. ................................................... 37

Fig. 53 - Resultados obtenidos para el Caso 2, curvas de nivel $B_{R}$.................................................. 37

Fig. 54 - Resultados obtenidos para el Caso 3, curvas de nivel $B_{R}$. ................................................... 37

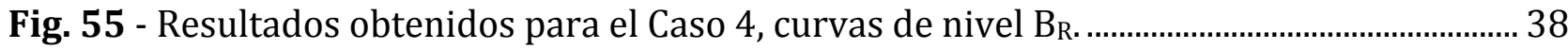

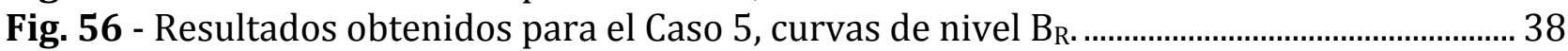

Fig. 57 - Resultados obtenidos para el Caso 6, curvas de nivel $B_{R}$.................................................. 38

Fig. 58 - Compensación con lazo activo ........................................................................................ 40

Fig. 59 - Flujo concatenado en un circuito externo, debido a la corriente "I", por un conductor " $r$ ".

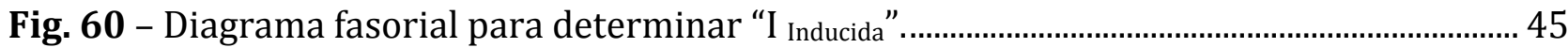

Fig. 61 - Flujos debido al campo original "Bo" y a la espira "Bi". ........................................................ 45

Fig. 62 - Conductores que forman dos lazos pasivos. ..................................................................... 46

Fig. 63 - Conductores que forman dos lazos pasivos, corte transversal........................................ 47

Fig. 64 - Esquema de utilización de las herramientas de cálculo .................................................... 50

Fig. 65 - Interfaz gráfica del módulo de cálculo de campo magnético............................................. 51

Fig. 66 - Resultados obtenidos con el programa............................................................................... 52

Fig. 67 - Interfaz gráfica, módulo de estimación de parámetros de lazo........................................ 53

Fig. 68 - Módulo de presentación de resultados, módulo de estimación de parámetros de lazo.

Fig. 69 - Arreglo de sistema trifásico con un lazo pasivo.

Fig. 70 - Arreglo para el circuito experimental, conductores de fase, lazo pasivo y elemento de medición...

Fig. 71 - Arreglo para el circuito experimental, conductores de fase, lazo pasivo y elemento de medición.

Fig. 72 - Arreglo para el circuito experimental, conductores de fase, lazo pasivo y elemento de medición.

Fig. 73 - Corrientes de fase y corriente por lazo, calculada y medida para secuencia RST..... 57

Fig. 74 - Perfiles obtenidos para el casos "1" y "2", con I=14,9A, valores medidos y calculados.58

Fig. 75 - Perfiles obtenidos para los casos “ 3 y 4", con I=14,9A, valores medidos y calculados.59

Fig. 76 - Perfiles obtenidos para los casos “0, 3, 5 y 6", con I=14,9A, valores medidos y calculados. Sección del conductor $90 \mathrm{~mm}^{2}$

Fig. 77 - Corrientes de fase y corriente por lazo, calculada y medida para secuencia directa e inversa utilizando conductores de $35 \mathrm{~mm}^{2}$......

Fig. 78 - Configuración utilizada para mediciones en laboratorio................................................. 61

Fig. 79 - Arreglo montado para mediciones, con cuatro lazos Pasivos.......................................... 62

Fig. 80 - Elementos para medición y control de la corriente.......................................................... 63 
Fig. 81 - Perfiles obtenidos para el caso "7" (Verde), valores medidos y calculados. Se incluyen los perfiles sin energizar la instalación (Azul) y sin LP (Rojo)..

Fig. 82 - Perfiles obtenidos para el caso "8" (Verde), valores medidos y calculados. Se incluyen los perfiles sin energizar la instalación (Azul) y sin LP (Rojo).

Fig. 83 - Perfiles obtenidos para el caso "9" (Verde), valores medidos y calculados. Se incluyen los perfiles sin energizar la instalación (Azul) y sin LP (Rojo).

Fig. 84 - Perfiles obtenidos para el caso "10" (Verde), valores medidos y calculados. Se incluyen los perfiles sin energizar la instalación (Azul) y sin LP (Rojo). 65

Fig. 85 - Perfiles obtenidos para el caso "11" (Verde), valores medidos y calculados. Se incluyen los perfiles sin energizar la instalación (Azul) y sin LP (Rojo).. 66

Fig. 86 - Perfiles obtenidos para el caso "12" (Verde), valores medidos y calculados. Se incluyen los perfiles sin energizar la instalación (Azul) y sin LP (Rojo). 66

Fig. 87 - Perfiles obtenidos para el caso "13" (Verde), valores medidos y calculados. Se incluyen los perfiles sin energizar la instalación (Azul) y sin LP (Rojo)............................66 66

Fig. 88 - Ubicación de lazos pasivos en la zona de empalmes, vista superior............................... 67

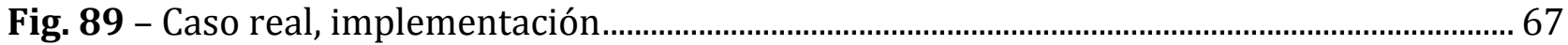

Fig. 90 - Disposición de lazos pasivos en planos por encima de los conductores de fase......... 68

Fig. 91 - Disposición de lazos pasivos en planos por encima y debajo de los conductores de fase.

Fig. 92 - Disposición de lazos pasivos en planos laterales a los conductores de fase................ 68

Fig. 93 - Disposición de lazos pasivos en el perímetro de los conductores de fase.................... 69

Fig. 94 - Ejemplo de aplicación, lazos pasivos ubicados en un plano paralelo al de los conductores de fase, entre estos y la zona de interés. Caso 1 - Tabla XV........................ 71

Fig. 95 - Perfiles de campo magnético con lazos pasivos en un plano horizontal entre los conductores de fase y la área de interés, I= $1000 \mathrm{~A}$, lazos de $185 \mathrm{~mm}^{2}$, Caso 1 - Tabla $\mathrm{XV}$.

Fig. 96 - Perfiles de FA con lazos pasivos en un plano horizontal entre los conductores de fase y la área de interés, I= $1000 \mathrm{~A}$, lazos de $185 \mathrm{~mm}^{2}$, Caso 1 - Tabla XV....................... 72

Fig. 97 - Ejemplo de aplicación, lazos pasivos ubicados en diferentes planos, todos los lazos tienen el mismo largo y ancho, Caso 2 - Tabla XV.

Fig. 98 - Perfiles de campo magnético con lazos pasivos en diferentes planos a ambos lados de los conductores de fase, I= $1000 \mathrm{~A}$, lazos de $185 \mathrm{~mm}^{2}$, Caso 2 - Tabla XV...

Fig. 99 - Perfiles de FA con lazos pasivos en diferentes planos a ambos lados de los conductores de fase, $\mathrm{I}=1000 \mathrm{~A}$, lazos de $185 \mathrm{~mm}^{2}$, Caso 2 - Tabla XV...

Fig. 100 - Valores de campo magnético en $\mathrm{x}=0 \mathrm{a}$, considerando lazos pasivos en un plano, Caso 1 - Tabla XV.

Fig. 101 - Valores de campo magnético en $\mathrm{x}=0$, considerando lazos pasivos a ambos lados de los conductores de fase, Caso 2 - Tabla XV.

Fig. 102 - Valores de FA en $\mathrm{x}=0$ a, considerando lazos pasivos en un plano, Caso 1 - Tabla XV.75

Fig. 103 - Valores de FA en $\mathrm{x}=0$ a, considerando lazos pasivos a ambos lados de los conductores de fase, Caso 2 - Tabla XV.

Fig. 104 - Valores de campo magnético en $\mathrm{x}=0$, Casos 1 a 5 - Tabla XV.......................................... 76

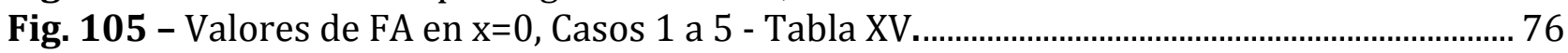

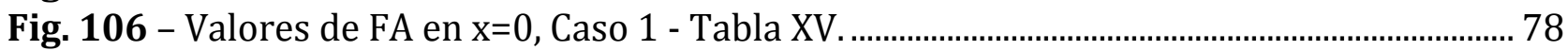

Fig. 107 - Valores de FA en $\mathrm{x}=0$, Caso 2 - Tabla XV ....................................................................... 78

Fig. 108 - LAAT 500 kV V-Arriostrada y Cross-Rope........................................................................... 78

Fig. 109 - LAAT 1 x $500 \mathrm{kV}$ - Caso 4

Fig. 110 - LAAT 1 x 500 kV - Caso 5................................................................................................. 79

Fig. 111 - Comparación del FA LAAT 500 kV .............................................................................. 80 
Fig. 112 - Perfiles de campo magnético, ejemplo del inciso 5.3.1 I=1000 A. 81

Fig. 113 - Valores de FA para diferentes alternativas de mitigación (Tabla XV), ejemplo del inciso 5.3.1 con $\mathrm{I}=1000 \mathrm{~A}$.

Fig. 114 - Variante del Caso 1 (LPH 1), ancho del LP interno $1 \mathrm{~m}$. 83

Fig. 115 - Variante del Caso 1 (LPH 2), ancho del LP interno 0,4 m 83

Fig. 116 - Valores de Campo magnético en función del número de lazos pasivos, Variantes del Caso 1, LPH 1 y 2. Sección de conductor de lazo 630 mm². 84

Fig. 117 - Valores de FA en función del número de lazos pasivos, Variantes del Caso 1, LPH 1 y 2. Sección de conductor de lazo $630 \mathrm{~mm}^{2}$. 84

Fig. 118 - Perfiles de Campo Magnético obtenidos para las variantes del Caso 1, corriente $1000 \mathrm{~A}$

\section{Lista de Tablas}

Tabla I - Subdivisión en el rango de frecuencias de hasta $100 \mathrm{kHz}$ [ 7 ] 5

Tabla II - Resumen de normativas y niveles de exposición para "B" a frecuencia industrial 10

Tabla III - Características constructivas de torres para LAAT de 2 x $220 \mathrm{kV}$ 13

Tabla IV - Características constructivas de torres para LAAT de $500 \mathrm{kV}$.

Tabla V - Alternativas de secuencia de energización para CSAT 2x132 kV, considerando zona de ductos y fosa de empalmes.

Tabla VI - Resumen de valores máximos de campo magnético y FA obtenidos, CSAT $2 \times 132 \mathrm{kV}$.

Tabla VII - Modelo experimental de espira y placa de blindaje, resumen de los valores experimentales.

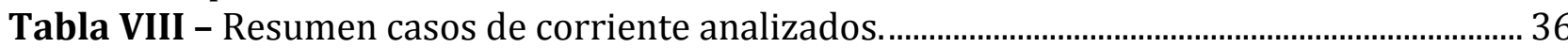

Tabla IX - Casos considerados ......................................................................................................... 55

Tabla X - Comparación entre valores calculados y medidos de corriente .................................... 57

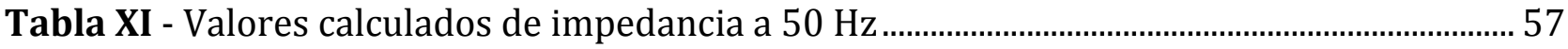

Tabla XII - Geometrías empleadas para las pruebas, en campo....................................................... 62

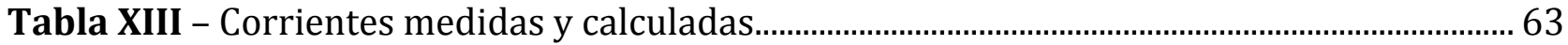

Tabla XIV - Características de conductores de BT considerados [ 44 ] ......................................... 69

Tabla XV - Configuraciones de lazos pasivos consideradas - Cables subterráneos.................... 70

Tabla XVI - Configuraciones de lazos pasivos consideradas - LAAT ................................................ 79 


\section{Lista de Siglas y Símbolos}

ADEERA: Asociación de Distribuidores de Energía Eléctrica de la República Argentina

AGEERA: Asociación de Generadores de Energía Eléctrica de la República Argentina

Al: $\quad$ Aluminio

ANEEL: Agéncia Nacional de Energía Elétrica

AT: $\quad$ Alta Tensión

ATEERA: Asociación de Transportistas de Energía Eléctrica de la República Argentina

B: $\quad$ Densidad de flujo magnético - (En la bibliografía suele identificarse a "B" como campo magnético.)

B $_{\mathrm{R}}$ : $\quad$ Módulo del campo magnético

BT: $\quad$ Baja Tensión

BX: $\quad$ Componente del campo magnético en el eje $\mathrm{x}$

By: $\quad$ Componente del campo magnético en el eje y

BZ: $\quad$ Componente del campo magnético en el eje $\mathrm{z}$

CH-AS: $\quad$ Coplanar Horizontal con Aisladores de Suspensión

CONICET: Consejo Nacional de Investigaciones Científicas y Técnicas

CR: Cross Rope

CS: $\quad$ Cable Subterráneo

CSAT: Cable Subterráneo de Alta Tensión.

CT: $\quad$ Centro de Transformación

CV-AS: $\quad$ Coplanar Vertical con Aisladores de Suspensión

CV-LP: $\quad$ Coplanar Vertical con Aisladores Line-Post

d.p.c.m: Decreto del Presidente del Consiglio dei Ministri

E: $\quad$ Intensidad del Campo Eléctrico

ELF: $\quad$ Extremely Low-Frequency

ENRE: Ente Nacional Regulador de la Electricidad

ET: Estación Transformadora

EU: $\quad$ European Union

FA: $\quad$ Factor de apantallamiento

FB: $\quad$ Frecuencias bajas

Fe: Hierro

FEB: $\quad$ Frecuencias Extremadamente Bajas

FMB: $\quad$ Frecuencias Muy Bajas

FV: $\quad$ Frecuencias de voz

H: Intensidad del Campo Magnético

I: $\quad$ Corriente eléctrica

IARC: International Agency on Research Cancer

ICNIRP: International Commission on Non-Ionizing Radiation Protection (Comisión Internacional de Protección contra Radiaciones No Ionizantes)

IEEE: Institute of Electrical and Electronics Engineers (Instituto de Ingenieros Electricistas y Electrónicos)

L: Inductancia

LA: Línea Aérea

LAAT: Línea Aérea de Alta Tensión

MT: $\quad$ Media Tensión 
MTESS: $\quad$ Ministerio de Trabajo, Empleo y Seguridad Social

NRPB: $\quad$ National Radiological Protection Board

OMS: $\quad$ Organización Mundial de la Salud

R: $\quad$ Resistencia eléctrica

RETIE: Reglamento Técnico de Instalaciones Eléctricas

RI: $\quad$ Radiaciones Ionizantes

RNI: Radiaciones No Ionizantes

SE: Secretaría de Energía de la Nación

U: $\quad$ Tensión eléctrica

UE: Unión Europea

VA: $\quad$-Arriostrada

WHO: World Health Organization

$\mathbf{X}_{\mathbf{L}}$ : Reactancia inductiva

Z: $\quad$ Impedancia

р: $\quad$ Profundidad de penetración

б: $\quad$ Conductividad eléctrica

Ф: $\quad$ Flujo de campo magnético

$\mu_{\mathbf{r}}$ : Permeabilidad relativa 


\section{Resumen}

El crecimiento de los centros urbanos y el aumento de la demanda de energía eléctrica hace que cada vez se requieran más instalaciones eléctricas en los centros urbanos. Asociados a las mismas se encuentran la generación de campos magnéticos, que desde ya hace varios años han despertado preocupación en la población por la asociación de estos con posibles efectos sobre la salud.

En algunas circunstancias resulta necesario disminuir los niveles de campo magnético generados. Para lograr dicho objetivo existen diferentes técnicas, como ser el incremento de la distancia en las zonas de interés o la aplicación de técnicas de mitigación más desarrolladas.

Desde el punto de vista técnico-económico, la utilización de lazos pasivos presenta una gran ventaja sobre otros métodos de mitigación, por lo que resulta de gran utilidad conocer la potencialidad de los mismos y aprovecharla en los casos en que resulte viable su aplicación.

El objetivo principal de este trabajo es desarrollar y validar experimentalmente herramientas que permitan analizar el efecto de mitigación que se obtiene en los valores de campo magnético generados por instalaciones de transmisión y distribución de energía eléctrica, al utilizar lazos pasivos principalmente en líneas aéreas y cables subterráneos.

En el desarrollo de la tesis, se presentan aspectos generales vinculados las fuentes de campos magnéticos existentes, focalizando las instalaciones eléctricas y en correspondencia las fuentes de frecuencia industrial.

Se realiza una evaluación de los valores admitidos de campo magnético, tanto a nivel nacional como internacional para estos valores de frecuencia, para lo cual se estudian normativas nacionales y recomendaciones publicadas por organizaciones reconocidas.

Se presentan valores de campo magnético generados en sistemas de transmisión y distribución de energía eléctrica, contemplando Líneas Aéreas, Cables Subterráneos, Estaciones Transformadoras y Centros de Transformación. Se presentan cálculos realizados con herramientas que permiten implementar modelos en dos y tres dimensiones, realizando a su vez comparaciones entre cálculos y mediciones.

Se estudian diferentes técnicas de mitigación de campo magnético utilizadas en instalaciones eléctricas. Las técnicas consideradas son el incremento de la distancia a las fuentes de campo magnético, diferentes alternativas de agrupamiento de conductores, cambios en la secuencia de energización para casos de líneas aéreas o cables subterráneos con más de una terna, se evalúa la utilización de materiales metálicos, lazos pasivos y lazos activos.

Considerando que en los centros urbanos se requieren más tendidos de cables subterráneos para abastecer la demanda de energía eléctrica y además que la instalación de los mismos se realiza en la vía pública, se enfoca el trabajo en la aplicación de lazos pasivos como herramienta de mitigación, ya que esta técnica presenta ventajas sobre otras estrategias de mitigación. Además en algunos casos esta técnica también puede ser aplicada para mitigar el campo magnético generado por líneas aéreas.

Para lograr el objetivo se estudian las diferentes variables intervinientes, como ser las características de los conductores a utilizar, el número de lazos y diferentes configuraciones. Para la implementación de esta técnica se desarrollaron las herramientas matemáticas correspondientes y un software de cálculo que permite realizar diferentes análisis. Se 
presentan comparaciones realizadas entre cálculos y mediciones en instalaciones montadas en laboratorio.

Se estudia la aplicación de lazos pasivos a instalaciones típicas de cables subterráneos y líneas aéreas, presentando finalmente las principales conclusiones obtenidas en este trabajo.

\section{Introducción}

La existencia de campos magnéticos, es una consecuencia del uso de la energía eléctrica, ya sea que sean generados por equipamientos o instalaciones domiciliarias o bien por instalaciones de las empresas del sector eléctrico.

Los valores de campos magnéticos máximos, correspondientes a instalaciones de las empresas eléctricas, se encuentran en las inmediaciones de estaciones transformadoras, líneas de transmisión, cables subterráneos, puestos de transición, líneas y cables de distribución, etc.

En el año 1979, los Campos Electromagnéticos de Frecuencia Extremadamente Bajas (CEMFEB) despertaron gran inquietud y alarma. Un informe publicado por un grupo de epidemiólogos, que indicaba una posible vinculación entre la leucemia infantil y ciertas configuraciones de líneas de transmisión, generó gran conmoción tanto en la comunidad científica como en la población [ 1 ].

La preocupación del público se centró principalmente en las líneas de transmisión de energía eléctrica, generadoras de campos eléctricos y magnéticos de frecuencias de $50 / 60 \mathrm{~Hz}$, ante la posibilidad de que la exposición a estos campos tuviera algún efecto nocivo para la salud.

En el ámbito científico numerosos han sido los estudios realizados hasta la fecha, tendientes a confirmar o rechazar tales efectos perjudiciales para la salud asociados con los CEM-FEB.

Los efectos de corto término en la salud humana debidos a la exposición a campos eléctricos y magnéticos de frecuencia industrial, son bien conocidos, el debate respecto de la existencia de efectos de largo término, especialmente cáncer, persiste a nivel mundial.

Con el objeto de evaluar los riesgos asociados, programas interdisciplinarios analizan los resultados de los estudios epidemiológicos y de laboratorio. En este sentido, en el año 2002 la Agencia Internacional de Investigación de Cáncer (IARC: International Agency for Research on Cancer), clasificó a los campos magnéticos de FEB en la Categoría 2B como "posiblemente carcinogénicos para los seres humanos" a partir de la evaluación de estudios publicados [ 2 ].

Existen recomendaciones, de carácter preventivo, realizadas por organismos internacionales y nacionales, como por ejemplo la Organización Mundial de la Salud (OMS o WHO: World Health Organization) y la Comisión Internacional sobre Protección ante Radiaciones No Ionizantes (ICNIRP: International Commission on Non Ionizing Radiation Protection). A su vez cada país, a través de los organismos afines dictan sus recomendaciones o reglamentaciones, que en muchos casos siguen los lineamientos indicados por la ICNIRP y la OMS [3] [ 4 ][5].

En la República Argentina la Secretaría de Energía (SE) es la encargada de establecer medidas de protección y control ambiental. Por otro lado, el Ente Nacional Regulador de la Electricidad (ENRE) es autoridad de fiscalización y control, por lo que establece procedimientos para el cumplimiento de normas y para este caso realiza auditorías ambientales.

Con el objeto de cumplir con parámetros ambientales en el área pública, los valores de campo magnético de frecuencia extremadamente baja (en el caso de las redes eléctricas 50 o 
$60 \mathrm{~Hz}$ ), deben permanecer por debajo de ciertos valores permitidos por la reglamentación vigente, en la que se indica que debe tratar de que los niveles de CEM teniendo en cuenta valores tan bajos como razonablemente alcanzables [ 6 ].

Por otra parte, las personas que tienen un mayor nivel de exposición son aquellas cuyos trabajos se desarrollan en el sector eléctrico, como pueden ser los operarios de redes eléctricas, estaciones trasformadoras o centrales de generación de energía eléctrica. En ambientes industriales adicionalmente pueden encontrarse exposiciones a campos cuyas frecuencias sean distintas a la industrial.

Un factor importante a considerar es la compatibilidad que existe entre los distintos componentes de los sistemas eléctricos entre si y entre estos y los diversos sistemas que pueden hallarse en el entorno. Como ejemplo de estos puede citarse la interferencia con cables de comunicaciones o equipamiento electrónico, cañerías u otras instalaciones metálicas paralelas a las redes eléctricas.

La interacción de los campos magnéticos con los materiales metálicos cumple un papel determinante, por lo que en muchos casos se recurre a soluciones que aprovechen esta interacción para lograr el objetivo de mitigar en forma efectiva el campo magnético. Dicha interacción puede causar efectos no deseados en algunas circunstancias, por lo que es necesario poner en práctica alguna técnica de mitigación y de este modo dar una solución al inconveniente.

La electricidad ocupa un lugar esencial en el estilo de vida moderno de nuestra sociedad y la utilización de la misma tiende a incrementarse con el paso del tiempo. Por otra parte, otro factor influyente es la indisponibilidad de espacios físicos para desarrollar la infraestructura que permita satisfacer el incremento de la demanda de energía, en particular se puede mencionar que no siempre es posible construir nuevas obras, con lo que resulta necesario modificar instalaciones existentes para ampliar la capacidad de transporte y/o distribución de energía eléctrica. Como consecuencia de estas situaciones, se incrementan los valores de campo magnético, pudiendo alcanzar valores de exposición inadmisibles, requiriendo en consecuencia la implementación de métodos de mitigación que resulten eficientes.

Como resultado de lo anteriormente señalado, surge la necesidad de explorar la implementación de sistemas de mitigación de campo magnético, lo que genera un desafío en innovar constantemente en las técnicas de mitigación. En este sentido resulta de gran interés conocer las diferentes técnicas de mitigación y los casos en que las mismas sean efectivas. Otro factor fundamental consiste en determinar cuál es la más conveniente desde el punto de vista técnico y económico.

Desde el punto de vista económico los lazos pasivos poseen una gran ventaja por sobre los otros métodos, pero estos no son aplicables en todos los casos y es por eso que es de gran utilidad conocer la potencialidad de los mismos y aprovecharla en los casos en que son aplicables.

\section{Aspectos generales}

\subsection{Fuentes de Campo Magnético}

Cuando se piensa en campos magnéticos, las barras magnéticas o imanes son probablemente los primeros elementos con que se los asocia, una aplicación típica es el empleo de sus propiedades en elementos de publicidad que se adhieren a las superficies metálicas. Hay que destacar que el campo magnético generado por estos es estático, o dicho de otro modo no se tratan de campos variables en el tiempo. 
Por otro lado la tierra en si es fuente de campo magnético, dependiendo de la localización presenta variaciones de 30 a $60 \mu \mathrm{T}$. Este campo no es completamente estático, sino que puede presentar algunas variaciones[11], entre las que se pueden mencionar:

- Reversiones geomagnéticas: el campo magnético de la Tierra cambia la polaridad imprevisiblemente, en la escala de tiempo geológico, debido a cambios bruscos en el movimiento de fluidos en el núcleo.

- Variaciones seculares: Las observaciones de campo magnético de la Tierra realizadas desde alrededor de 400 años, muestran un cambio gradual en la posición de los polos magnéticos.

- Variaciones diurnas: Los cambios diarios en el campo se deben a cambios en las corrientes de partículas cargadas en la ionosfera. Estos cambios son leves y en promedio están alrededor de 50 nT.

- Tormentas geomagnéticas: Perturbaciones de corto plazo en el campo magnético están asociadas con la actividad de las manchas solares y las corrientes de partículas cargadas provenientes del sol. Estas variaciones pueden ser de hasta $1.000 \mathrm{nT}$ y pueden inducir importantes corrientes de secuencia cero en líneas aéreas largas, además son capaces de causar saturación en transformadores, disparos e incluso apagones.

En la actualidad se pueden encontrar campos magnéticos variables en el tiempo de distintas frecuencias, estos se hallan asociados a las corrientes que los originan. Dependiendo de la aplicación, se pueden encontrar corrientes de diversas frecuencias. En particular, para este trabajo, interesan los campos de frecuencia industrial $(50 \mathrm{~Hz})$, que tienen su origen en la circulación de corriente de la misma frecuencia por las redes e instalaciones de las empresas del sector eléctrico. En la bibliografía especializada, a esta frecuencia se la ubica en el rango de Frecuencias Extremadamente Bajas (FEB), lo mismo ocurre en las redes que operan en 60 $\mathrm{Hz}$.

En la Figura 1 se aprecia un esquema en donde se presenta el espectro electromagnético, las fuentes considerando diferentes actividades y la frecuencia de operación, los efectos que se producen y la división entre Radiaciones Ionizantes (RI) y Radiaciones No Ionizantes (RNI).

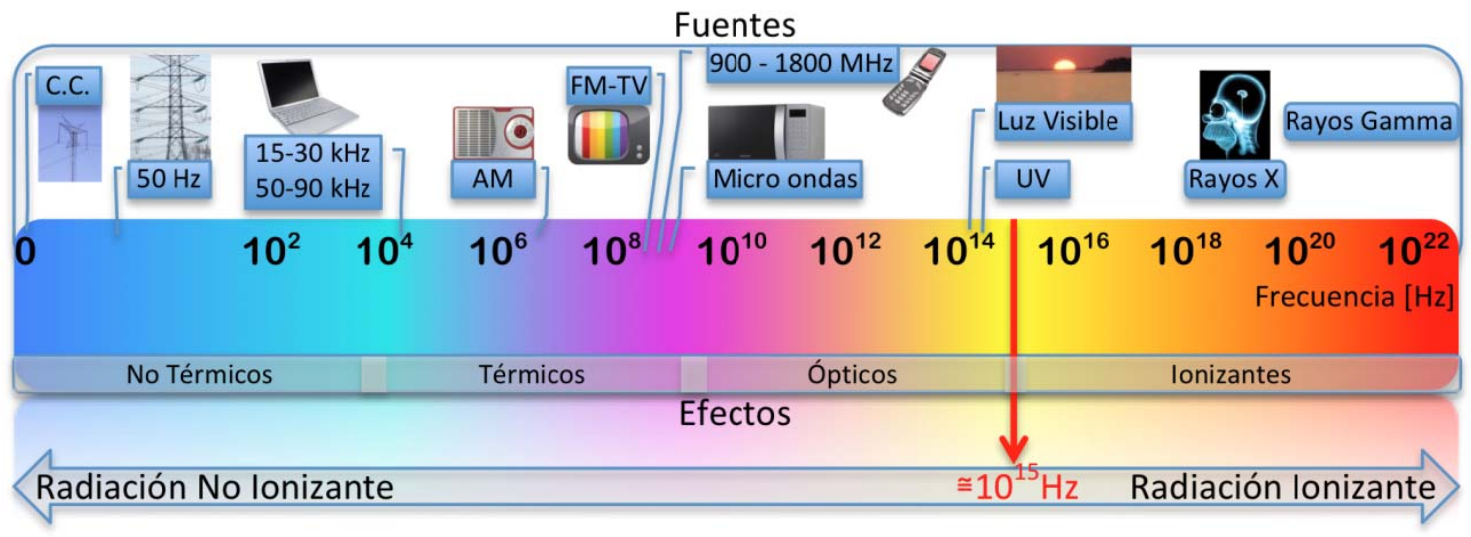

Fig. 1 - Espectro electromagnético 


\subsection{Rangos de frecuencias}

En la Tabla I se indica una subdivisión de rangos de frecuencia, contemplando los campos estáticos y campos variables en el tiempo hasta las frecuencias bajas. Hay que destacar que hasta este rango de frecuencias es posible tratar por separado a los campos eléctricos y los magnéticos.

Tabla I - Subdivisión en el rango de frecuencias de hasta 100 kHz [ 7 ]

\begin{tabular}{|c|c|}
\hline Rango de frecuencia [kHz] & Nombre de la subdivisión \\
\hline 0 & Estático \\
\hline$>0-0,3$ & Frecuencias Extremadamente Bajas (FEB) \\
\hline $0,3-3$ & Frecuencias de voz (FV) \\
\hline $3-30$ & Frecuencias muy bajas (FMB) \\
\hline $30-100$ & Frecuencias bajas (FB) \\
\hline$*$ frecuentemente el límite más bajo se toma en $0,03 \mathrm{kHz}=30 \mathrm{~Hz}$ \\
\hline
\end{tabular}

Con respecto a la frecuencia de los sistemas eléctricos se puede decir que durante el siglo XX, existieron redes con al menos cinco frecuencias diferentes introducidas en la década de 1890. Estas incluían $25 \mathrm{~Hz}$ (en las Cataratas del Niágara), $30 \mathrm{~Hz}$ (introducida por la Westinghouse Electric Company), $40 \mathrm{~Hz}$ (introducida por General Electric), $50 \mathrm{~Hz}$ (utilizado en el sur de California y en Europa) y $60 \mathrm{~Hz}$ (en diferentes zonas de Estados Unidos) [ 8 ].

También existieron redes de distribución que operaron en Corriente Continua, como ejemplo se puede citar la red introducida en la ciudad de Nueva York en septiembre del año 1882, que estuvo en operación hasta noviembre de 2007, cuando se desconectaron los últimos cinco usuarios [ 9 ]. Actualmente esta opción se utiliza para la transmisión de energía a largas distancias, como ejemplo puede mencionarse la red en $600 \mathrm{kV}$ existente entre los estados de Paraná (Itaipu) y San Pablo en Brasil.

La frecuencia a la que se genera electricidad en corriente alterna se conoce como "Frecuencia Industrial". Las instalaciones de generación, transporte y distribución de energía eléctrica, en la República Argentina y en la mayor parte del mundo, operan a la frecuencia de $50 \mathrm{~Hz}$. La frecuencia de $60 \mathrm{~Hz}$ es utilizada en América del Norte, Brasil y en algunas zonas de Japón.

Se destaca que las redes operan en el rango de las FEB, por lo que se centra la atención en este rango de frecuencia, particularmente se consideran en este trabajo instalaciones que operan en $50 \mathrm{~Hz}$, frecuencia de operación del sistema eléctrico Argentino.

\subsection{Definiciones}

Frecuencias Extremadamente Bajas "FEB": Rango de frecuencias que se encuentran por encima de los campos electromagnéticos estáticos $(0 \mathrm{~Hz})$ y hasta los $300 \mathrm{~Hz}$. Frecuentemente se define como el límite inferior a la frecuencia de $30 \mathrm{~Hz}$ [ 7 ].

Densidad de flujo magnético "B": Es la magnitud vectorial de divergencia cero en todos los puntos, que determina la componente de la fuerza de Coulomb-Lorentz, que es proporcional a la velocidad de los portadores de carga [ 10 ].

La unidad utilizada por el Sistema Internacional de Unidades (SIU) y por el Sistema Métrico Legal Argentino (SIMELA) es el tesla [T] 
Público en general: El término público en general se refiere a toda la población, incluye a las personas de todas las edades y con diferente estado de salud, y esto incluye en particular los grupos o personas vulnerables, como los débiles, los trabajadores de edad avanzada, embarazadas, bebés y niños pequeños [ 3 ].

Exposición del público en general: Toda exposición a campos de baja frecuencia recibida por los miembros del público en general. Esta definición excluye a la exposición ocupacional y exposición médica [ 3 ].

Factor de apantallamiento [FA]: es la relación entre la densidad de flujo magnético en un punto dado $(\mathrm{P})$ en ausencia $\left(\mathrm{B}_{0}\right)$ y en presencia $\left(\mathrm{B}_{\mathrm{M}}\right)$ de mitigación. Este factor se define de acuerdo con la Ec. 1, [11].

$$
F A=\frac{B_{0}(P)}{B_{M}(P)}
$$

donde:

$\mathrm{B}_{0}$ : es el valor (rms) del campo magnético sin la utilización de mitigación.

$\mathrm{B}_{\mathrm{M}}$ : es el valor (rms) del campo magnético con la utilización de mitigación.

Es posible encontrar en la bibliografía la utilización del FA definido como la inversa del presentado en la Ec. 1 [ 12 ], en la Ec. 2 se presenta dicha definición alternativa.

$$
F A^{*}=\frac{B_{M}(P)}{B_{0}(P)}
$$

Efectividad del apantallamiento: este factor se define de acuerdo con la Ec. 3.

$$
E A=(1-(1 / F A)) \times 100
$$

\section{Valores admitidos de campo magnético}

En este punto se tratan aspectos vinculados a los límites de exposición de campo magnético recomendados por organismos internacionales, como la OMS, la ICNIRP, el IEEE o la Unión Europea.

Por otro lado se realiza un análisis de la legislación vigente en la República Argentina, para el público en general y para exposición ocupacional.

Por último se hace una síntesis de los valores límites adoptados por diferentes países en sus reglamentaciones.

En el Anexo I se presentan más detalles sobre los criterios considerados por las organizaciones internacionales utilizados para establecer los valores de referencia.

\subsection{Recomendaciones a nivel internacional}

Existen publicaciones realizadas por entidades internacionales, como ser la Organización Mundial de la Salud (OMS o WHO: World Health Organization), Comisión Internacional de Protección contra Radiaciones No Ionizantes (ICNIRP: International Commission on NonIonizing Radiation Protection), Instituto de Ingenieros Electricistas y Electrónicos (IEEE: Institute of Electrical and Electronics Engineers) o la Unión Europea. Algunas de estas publicaciones son recomendaciones que cada país puede adoptarlas a través de las correspondientes entidades competentes. 
Realizando un análisis cronológico de las publicaciones existentes se identifican a continuación las más importantes.

En el año 1998, la ICNIRP publica la Guía para los límites de exposición de campos eléctricos, magnéticos y electromagnéticos variables con el tiempo, con frecuencias hasta de $300 \mathrm{GHz}$. Los valores límites recomendados por ICNIRP, consideran los efectos de corto término, para lo cual establece que no se debe superar la densidad de corriente inducida en el cuerpo. Para la frecuencia de $50 \mathrm{~Hz}$, establece que la densidad de corriente para el público en general es de $2 \mathrm{~mA} / \mathrm{m}^{2}$, mientras que para ocupacional es de $10 \mathrm{~mA} / \mathrm{m}^{2}$. Estos límites definen que los valores de campo eléctrico y magnético para la población son $5 \mathrm{kV} / \mathrm{m}$ y $100 \mu \mathrm{T}$, respectivamente, mientras que a nivel ocupacional son de $10 \mathrm{kV} / \mathrm{m}$ y $500 \mu \mathrm{T}$ [ 4 ].

La Unión Europea (European Union) recomendó en el año 1999 a los estados miembros, el uso de las guías de ICNIRP para la limitación de la exposición a CEM para el público en general (1999/519/CE).

En el año 2002 IARC y OMS publican "Monographs on the Evaluation of Carcinogenic Risks to HumansNon-Ionizing Radiation, Part 1: Static and Extremely Low-Frequency (ELF) Electric and Magnetic Fields" Volume 80 (2002) [ 2 ]. En la misma se clasifica a los campos magnéticos de FEB como "posiblemente cancerígenos para los seres humanos" (Categoría 2B). Sin embargo, esta evidencia No es lo suficientemente contundente como para recomendar cambios en las guías de ICNIRP

En el mismo año la OMS, publica la Nota Descriptiva 263, señalando que los campos electromagnéticos de FEB son categoría “2B" basados en estudios epidemiológicos de leucemia en niños. Esta es la más débil de tres categorías para clasificar agentes carcinógenos potenciales basados en evidencia científica publicada [ 5 ].

En el año 2002 el IEEE publicó la Norma IEEE StdC95.6-2002 [ 13 ] que define como criterio de restricción básica el valor límite de corriente inducida que evite efectos adversos. Por ello se establecen diferentes valores para diferentes partes del cuerpo. Como resultado de estas restricciones para el público en general se establecen límites de $5 \mathrm{kV} / \mathrm{m}$ para el campo eléctrico y $904 \mu \mathrm{T}$ para el flujo de inducción magnética, a $50 \mathrm{~Hz}$. Para ambientes controlados se recomiendan límites de $20 \mathrm{kV} / \mathrm{m}$ para el campo eléctrico y $2710 \mu \mathrm{T}$ para el flujo de inducción magnética, a $50 \mathrm{~Hz}$.

En el año 2004 la Comisión Europea recomendó, a través de la Directiva 2004/40/CE, a los estados miembros el uso de las guías de ICNIRP para la limitación de la exposición a CEM para la exposición laboral [ 14 ].

En el año 2007, la OMS, en el marco del Proyecto Efectos Sanitarios y Ambientales de la Exposición a Campos Eléctricos y Magnéticos Variables con el Tiempo, publica:

- Serie Criterios de Salud Ambiental, Vol. 238. Extremely low frequency fields. Ginebra, Organización Mundial de la Salud. 2007 [ 15 ].

- Nota descriptiva N³22: Campos electromagnéticos y salud pública. Exposición a campos de frecuencia extremadamente baja. Junio de 2007 [ 16 ].

Con respecto a las medidas de protección, el Vol. 238 de la OMS [ 15 ], indica lo siguiente:

Solamente se han establecido los efectos agudos, y hay dos recomendaciones internacionales de límites de exposición (ICNIRP-1998 [ 4 ]; IEEE-2002 [ 13 ]) destinadas a la protección frente a estos efectos.

Además de estos efectos agudos conocidos, hay incertidumbres acerca de la existencia de efectos crónicos, debido a que las pruebas de una vinculación entre la exposición a campos 
magnéticos de FEB y la leucemia infantil son limitadas. Por consiguiente, se justifica la utilización de enfoques de precaución. Sin embargo, no se recomienda la reducción de los valores límites de las recomendaciones sobre la exposición a algún nivel arbitrario en aras de la precaución. Dicha práctica socava el fundamento científico en el que se basan los límites y probablemente sea una manera costosa y no necesariamente eficaz de proporcionar protección.

La aplicación de otros procedimientos apropiados de precaución para reducir la exposición es razonable y se justifica. Sin embargo, la energía eléctrica aporta evidentes beneficios a la salud, sociales y económicos, y los enfoques de precaución no deberían comprometer esos beneficios. Además, teniendo en cuenta, por una parte la debilidad de la evidencia de una vinculación entre la exposición a campos magnéticos de FEB y la leucemia infantil y por otra parte el limitado impacto en la salud pública si existe una vinculación, no están claros los beneficios para la salud de una reducción de la exposición. Así pues, los costos de las medidas de precaución deberían ser muy bajos. Los costos de la aplicación de reducciones de la exposición varían de un país a otro, por lo que resulta muy difícil formular una recomendación general para alcanzar un equilibrio entre los costos y los posibles riesgos derivados de los campos de FEB.

En vista de lo expuesto, se recomienda lo siguiente.

Los encargados de formular las políticas deberían establecer recomendaciones para la exposición a campos de FEB tanto del público en general como de los trabajadores. La mejor fuente de orientación para los niveles de exposición y los principios aplicables a la revisión científica son las recomendaciones internacionales.

En el año 2009 ICNIRP publica recomendaciones para exposición a campos magnéticos estáticos, en donde se recomienda un nivel de $400 \mathrm{mT}$ para exposición del público en general considerando cualquier parte del cuerpo, además se señala que para personas con implantes electrónicos o que contengan materiales ferromagnéticos se debería adoptar un límite de 0,5 mT. Para exposición ocupacional los valores recomendados son de $2 \mathrm{~T}$ considerando cabeza y torso y $8 \mathrm{~T}$ para extremidades. Para el caso del campo eléctrico estático, ICNIRP no ha recomendado límites de exposición [ 17 ].

En el año 2010, ICNIRP publica la Guía para los límites de exposición de campos magnéticos variables con el tiempo, con frecuencias en el rango de $1 \mathrm{~Hz}$ a $100 \mathrm{kHz}$. El principal cambio con respecto a las guías previas se basa en recomendar los valores límites, considerando el campo eléctrico interno inducido, en lugar de la densidad de corriente inducida en el cuerpo, ya que es la magnitud física que determina los efectos biológicos. Además, se consideran los únicos efectos comprobados en voluntarios expuestos a campos magnéticos de baja frecuencia, que son la estimulación de tejidos del sistema nervioso central y periférico y la inducción en la retina de fosfenos, que son una percepción visual de luz centellante en la periferia del campo visual. La retina forma parte del sistema nervioso central y es considerada como un modelo adecuado, para los efectos del campo eléctrico inducido en el circuito neuronal del sistema nervioso central en general.

Para la frecuencia de $50 \mathrm{~Hz}$, ICNIRP 2010 establece que el campo eléctrico interno inducido para el público en general es de $0,02 \mathrm{~V} / \mathrm{m}$., mientras que para ocupacional es de $0,1 \mathrm{~V} / \mathrm{m}$. Estas restricciones definen que los valores límites de exposición de campo magnético para el público en general es $200 \mu \mathrm{T}$ y a nivel ocupacional es de $1 \mathrm{mT}$. Los valores límites de campo eléctrico para $50 \mathrm{~Hz}$ son de $5 \mathrm{kV} / \mathrm{m}$ para público en general y $10 \mathrm{kV} / \mathrm{m}$ para ocupacional [ 3 ]. 


\subsection{Regulación Argentina}

En nuestro país, en el marco de la ley 24065, es rol de la Secretaría de Energía de la Nación (SE) el fijar medidas de protección y control ambiental, estableciendo a su vez los estándares ambientales. Mientras que el Ente Nacional Regulador de la Electricidad (ENRE), debe establecer los procedimientos para el cumplimiento de normas, realizar auditorías ambientales y es autoridad de fiscalización

En el año 1996 se registraron severos conflictos en Argentina, cuando la población se opuso a la instalación de nuevas líneas de transmisión (en $132 \mathrm{kV}$ ) en zonas del Gran Buenos Aires (Ezeiza, Isidro Casanova y La Matanza). En años siguientes nuevos conflictos aparecieron en el interior del país, manifestándose en localidades como La Plata, Ensenada, Chacabuco, Posadas, Mendoza y otras localidades. En la mayoría de los casos, la expresión popular logró, inclusive, la suspensión de las obras.

Conflictos de tal naturaleza, requirieron de la inmediata actuación de las autoridades nacionales en cuanto a la elaboración de una normativa de diseño de los sistemas de transmisión y distribución de energía eléctrica, que contemplara los posibles efectos en la salud pública, así como también los estándares de protección ambiental y pública.

Con este objetivo la SE solicitó, en el año 1996, la realización de una exhaustiva evaluación del estado de las investigaciones a nivel mundial en cuanto a los aspectos sanitarios, así como también a la normativa existente sobre niveles de exposición a campos eléctricos y magnéticos de los sistemas eléctricos.

De dicho análisis surgió que, siendo insuficientes e inconsistentes los datos científicos que permitieran establecer, y mucho menos cuantificar el riesgo para la salud asociado a la exposición a CEM-FEB, y tomando como base la experiencia de reglamentación a nivel mundial, la SE desarrolla una resolución que abarca los aspectos "ambientales" de los electroductos en su conjunto y no una normativa "sanitaria". Estos aspectos son: ocupación del espacio, impacto visual, efecto corona (radiointerferencia y ruido audible), campo eléctrico y campo de inducción magnética y atienden a establecer la compatibilidad de las instalaciones de transmisión y distribución con el ambiente.

El documento generado en el año 1996, fue la base para la realización de una normativa, dando intervención para su la elaboración a la Secretaria de Política y Regulación de Salud del Ministerio de Salud y Acción Social, al Consejo Nacional de Investigaciones Científicas y Técnicas (CONICET) de la Secretaria de Ciencia y Tecnología, a la Secretaria de Recursos Naturales y Desarrollo Sustentable dependiente de Presidencia de la Nación, ENRE, a la Asociación de Generadores de Energía Eléctrica de la República Argentina (AGEERA), a la Asociación de Distribuidores de Energía Eléctrica de la República Argentina (ADEERA), a la Asociación de Transportistas de Energía Eléctrica de la República Argentina (ATEERA).

La normativa fue publicada en el año 1998 como Resolución SE 77/98 [ 6 ]. Respecto a las consideraciones, tanto para campo eléctrico como para campo de inducción magnética, la resolución resulta de carácter interina y está dirigida a evitar aumentos innecesarios de los niveles existentes de exposición a campos eléctricos y magnéticos. La misma no indica niveles de exposición seguros, ni niveles inseguros y se aplica solamente a instalaciones de transmisión futuras a su puesta en vigencia.

Los valores límites publicados resultan: para campo eléctrico (E) $3 \mathrm{kV} / \mathrm{m}$, y para campo de inducción magnética (B) $25 \mu \mathrm{T}$ medidos a $1 \mathrm{~m}$ del nivel del suelo, en el borde de la franja de servidumbre, fuera de ella y borde perimetral de estaciones transformadoras. 
Por otra parte el organismo responsable del contralor de esta reglamentación es el ENRE, quien actúa por un lado evaluando los estudios de impacto ambiental presentado en los estudios de acceso a la capacidad de transporte, ante la incorporación de nuevas instalaciones en la red eléctrica, y por otra parte realiza el control de las instalaciones existentes, contemplando auditorías y evaluando los informes de Gestión Ambiental.

Debe destacarse que al momento de la publicación en el boletín oficial de la Res. SE 77/1998, 18 de marzo de 1998, ICNIRP no había publicado la recomendación correspondiente al mismo año. Dicha publicación fue realizada en Health Physics en abril de 1998 [ 4 ].

Con respecto a la exposición ocupacional, la Ley $N^{\circ} 19587 / 72$ "Seguridad e Higiene en el trabajo" [ 18 ] es la que regula las condiciones que deben cumplirse en ambientes laborales. La Resolución 295/2003 del Ministerio de Trabajo, Empleo y Seguridad Social (MTESS) aprueba las especificaciones técnicas sobre radiaciones, que como Anexo II forma parte integrante de dicha Resolución. Allí se indican los valores de exposición máximos para el Campo eléctrico de $25 \mathrm{kV} / \mathrm{m}$ (de 0 a $100 \mathrm{~Hz}$ ) y para el campo de inducción magnética $1200 \mu \mathrm{T}$ (a $50 \mathrm{~Hz})[19$ ].

\subsection{Regulaciones adoptadas por diferentes países}

En general pueden diferenciarse tres grupos: países que adoptan valores superiores a los indicados por ICNIRP en 1998, países que adoptan los mismos valores y países que adoptan valores inferiores. Debe notarse además que la OMS recomienda los valores indicados por ICNIRP en 1998, al día de la fecha no se conoce algún país que haya adoptado los valores indicados por ICNIRP en 2010.

En la Tabla II se realiza un resumen en donde se identifican diferentes países u organizaciones internacionales, sus respectivas normativas, los valores adoptados límites para "B" a frecuencia industrial y observaciones para cada caso en particular.

Tabla II - Resumen de normativas y niveles de exposición para "B" a frecuencia industrial

\begin{tabular}{|c|c|c|c|c|}
\hline País/Org. & Normativa & \multicolumn{2}{|c|}{$\begin{array}{c}\text { B límite }[\mu \mathrm{T}] \\
\text { aplicado a exposición: }\end{array}$} & Observaciones \\
\hline \multirow{2}{*}{ ICNIRP } & \multirow{2}{*}{$1998[4]$} & Pública & 100 & \\
\hline & & Ocupacional & 500 & \\
\hline \multirow{2}{*}{ ICNIRP } & \multirow{2}{*}{$2010[3]$} & Pública & 200 & \\
\hline & & Ocupacional & 1000 & \\
\hline \multirow{2}{*}{ IEEE } & \multirow{2}{*}{ C95.6-2002 [ 13 ] } & Pública & 904 & \\
\hline & & Ocupacional & 2710 & \\
\hline \multirow{2}{*}{ Colombia } & \multirow{2}{*}{ RETIE [ 20 ] } & Pública & 100 & Frec. $60 \mathrm{~Hz}$ \\
\hline & & Ocupacional & 500 & \\
\hline \multirow{2}{*}{ Brasil } & \multirow{2}{*}{ ANEEL RS № 398/2010 [ 21 ] } & Pública & 83,33 & Frec. $60 \mathrm{~Hz}\left({ }^{*}\right)$ \\
\hline & & Ocupacional & 416,7 & \\
\hline \multirow{2}{*}{$\begin{array}{l}\text { Reino } \\
\text { Unido }\end{array}$} & \multirow{2}{*}{ NRPB vol. 15 № 2/2004 [ 22 ] } & Pública & 100 & \\
\hline & & Ocupacional & 500 & \\
\hline Argentina & Res. SE 77/1998 [ 6 ] & Pública & 25 & \\
\hline
\end{tabular}




\begin{tabular}{|c|c|c|c|c|}
\hline \multirow[t]{2}{*}{ País/Org. } & \multirow{2}{*}{$\begin{array}{c}\text { Normativa } \\
\text { Res. MTESS } 295 \text { / } 2003 \text { [ 19 ] }\end{array}$} & \multicolumn{2}{|c|}{$\begin{array}{c}\text { B límite }[\mu \mathrm{T}] \\
\text { aplicado a exposición: }\end{array}$} & \multirow[t]{2}{*}{ Observaciones } \\
\hline & & Ocupacional & 1200 & \\
\hline \multirow{2}{*}{ Italia } & \multirow{2}{*}{$\begin{array}{l}\text { d.p.c.m } 8 \text { julio/2003 [ } 23 \text { ] [ } \\
24 \text { ] }\end{array}$} & Pública & 3 & $(*)$ \\
\hline & & Ocupacional & - & \\
\hline \multirow{2}{*}{ Suiza } & \multirow{2}{*}{ NISV/1999 [ 25 ] } & Pública & 1 & \\
\hline & & Ocupacional & - & \\
\hline
\end{tabular}

\section{Campos magnéticos en sistemas de transmisión y distribución de energía eléctrica}

\subsection{Cálcullos de campo magnético en 2D y en 3D.}

Los modelos bidimensionales pueden arrojar buenos resultados para algunas instalaciones como ser Líneas Aéreas de Alta Tensión (LAAT) y Cables Subterráneos (CS), ya que la componente de campo magnético en la dirección de la corriente se anula.

Las Líneas Aéreas describen una catenaria entre dos puntos de fijación, por lo que para conocer el campo magnético a lo largo de un vano habría que representar la correspondiente curva. Esto requiere de la elaboración de modelos tridimensionales.

Sin embargo para el cálculo, es posible realizar una simplificación, si se considera que los conductores se disponen a lo largo del eje "Z", se emplea un modelo de conductor rectilíneo de longitud infinita, pero cuya altura coincide con la de la catenaria en la coordenada "Z" donde se desea calcular el campo.

En el Anexo II se presentan las ecuaciones que se utilizan para el cálculo del campo magnético en líneas aéreas, considerando la geometría de los conductores, desbalances de corrientes y características del terreno.

En el Anexo III se trata el fenómeno de polarización del campo magnético. A su vez se ha desarrollado un programa para realizar estos cálculos.

Para el cálculo en 3D se utiliza el programa Opera 10.506 de la empresa Vector Fields Inc. Este software permite el modelado en tres dimensiones de todos los conductores como segmentos finitos de corriente. Los campos calculados siguen las recomendaciones de la norma para medidores de tres bobinas, por ser estos más representativos del mismo. Para determinar $B_{R}$, en una simulación, primero se debe calcular el valor eficaz de cada una de las tres componentes espaciales que definen al campo: $\mathrm{B}_{\mathrm{X}}, \mathrm{B}_{\mathrm{Y}} \mathrm{y} \mathrm{B}_{\mathrm{Z}}$. Esto se logra sumando el aporte de cada uno de los segmentos de corriente respetando la amplitud y fase relativa de su variación en el tiempo. Una vez obtenidos los valores eficaces $B_{X}, B_{Y}$ y $B_{Z}$ numéricamente, se los combina para obtener el módulo con las tres componentes.

Los resultados son presentados en forma de mapas o curvas de nivel de campo magnético, obtenidos a un metro de altura sobre el terreno.

En el Anexo IV se presenta una descripción del software comercial utilizado para realizar los cálculos. 
En el Anexo V se presentan los datos correspondientes a las diversas configuraciones de líneas aéreas y cables subterráneos, para los cuales se realizan los cálculos de campo magnético, indicando las cotas y distancias necesarias para ello. Para el caso de las líneas aéreas, la altura del conductor al terreno se indica en cada caso en el cuerpo principal de la Tesis.

\subsection{Campo Magnético generado por Líneas Aéreas}

En este inciso se presentan algunos ejemplos, en los que se analizan los niveles de campo magnético producidos por LAAT. Se consideran estructuras típicas existentes en la República Argentina y luego, a través de herramientas de simulación se calcularon los niveles de campo magnético, se obtuvieron los perfiles de campo a $1 \mathrm{~m}$ sobre el nivel del suelo.

Los casos analizados se presentan en la Fig. 2, en la que se aprecian los diferentes tipos constructivos de LAAT 2 x 220kV utilizados en República Argentina. En a) se presenta una imagen en la que la doble terna se encuentra en la configuración coplanar horizontal, en la misma se aprecian aisladores en cadenas de suspensión en $\mathrm{V}$ y en los conductores externos cadenas simples (CH-AS). En b) se presenta una estructura con cadenas aisladores de suspensión dobles, la doble terna se encuentra en la configuración coplanar vertical (CV-AS). En c) se presenta una estructura en la que se emplean aisladores tipo Line-Post, la doble terna se encuentra en la configuración coplanar vertical (CV-LP). Esta es la más compacta de las líneas analizadas en este punto.

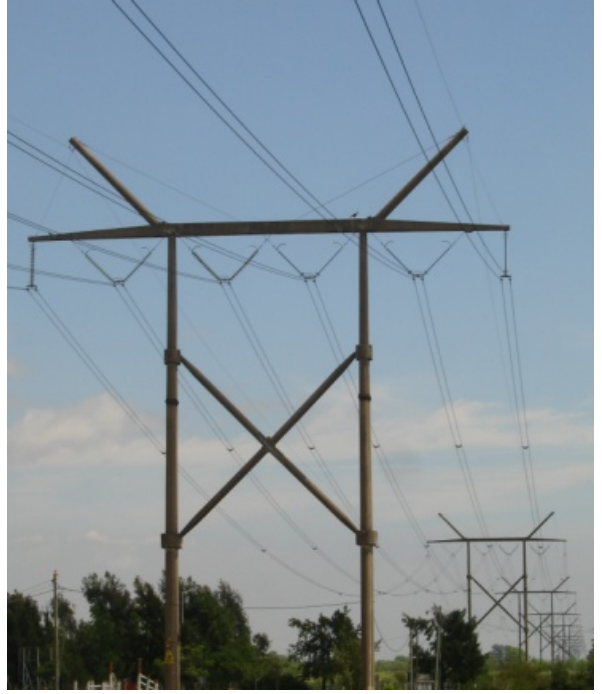

a) Coplanar horizontal, con aisladores de suspensión (CH-AS).

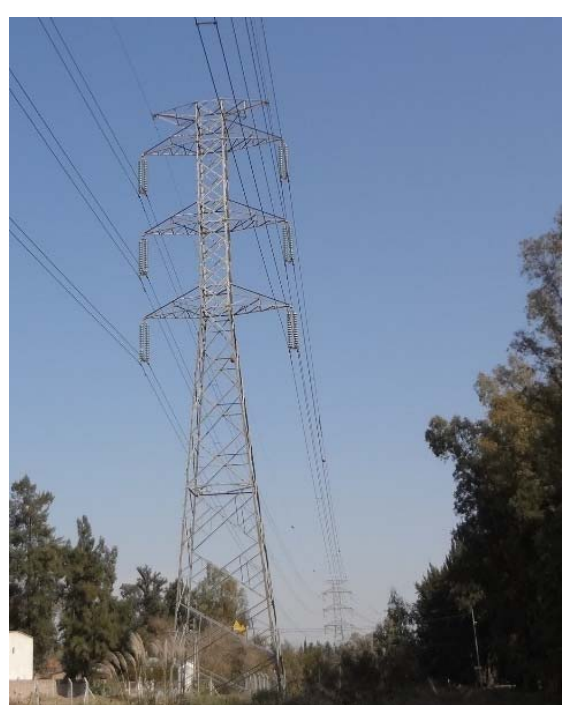

b) Coplanar vertical, con aisladores de suspensión (CV-AS).

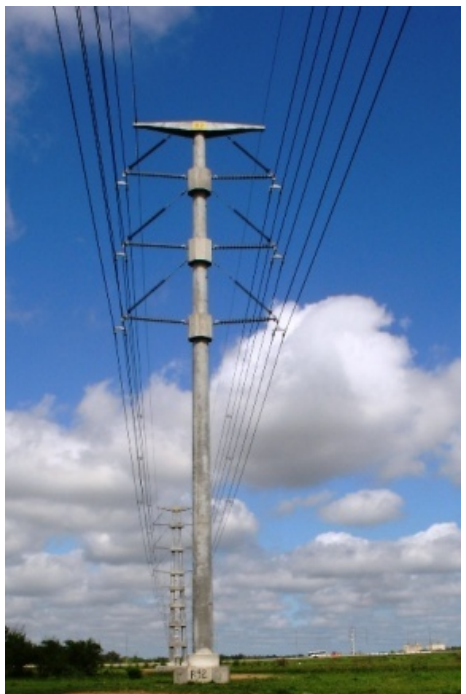

c) Coplanar vertical, con aisladores Line-Post (CV-LP).

Fig. 2 - Configuraciones de LAAT 2x220kV.

En la Tabla III se presentan las principales dimensiones de las estructuras consideradas en este inciso, como ser las alturas de los conductores inferiores, distancias entre conductores externos, etc. 
Tabla III - Características constructivas de torres para LAAT de 2 x $220 \mathrm{kV}$

\begin{tabular}{|c|c|c|c|}
\hline \multirow{2}{*}{ Dimensión } & \multicolumn{3}{|c|}{ Torre } \\
\cline { 2 - 4 } & CH-AS & CV-AS & CV-LP \\
\hline Altura de conductores inferiores en torre [m] & 13 & 21,5 & 12,6 \\
\hline Altura de conductores inferiores en el vano [m] & 10,2 & 10,2 & 10,2 \\
\hline Altura de torre [m] & 25,6 & 33,5 & 21,5 \\
\hline Distancia entre conductores externos [m] & 28 & 7 & 6 \\
\hline
\end{tabular}

En la Fig. 3 se presentan los resultados obtenidos para las tres configuraciones consideradas.

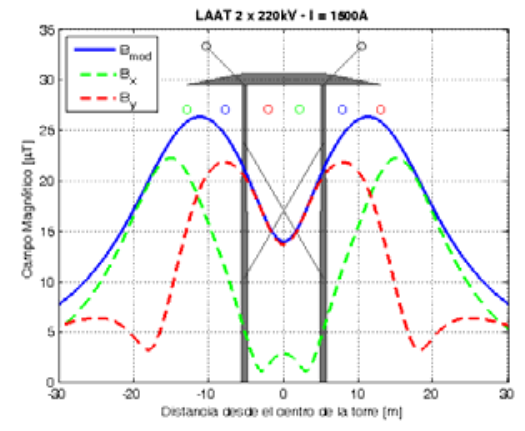

a) CH-AS.

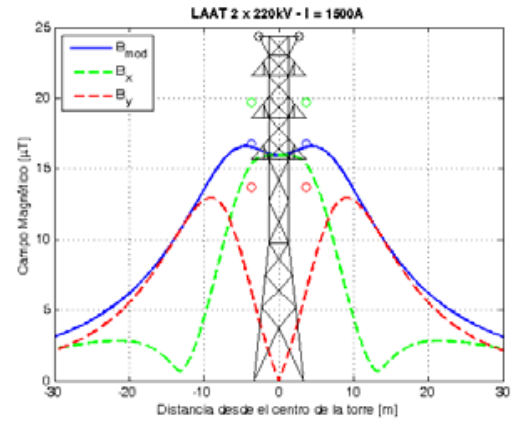

b) CV-AS.

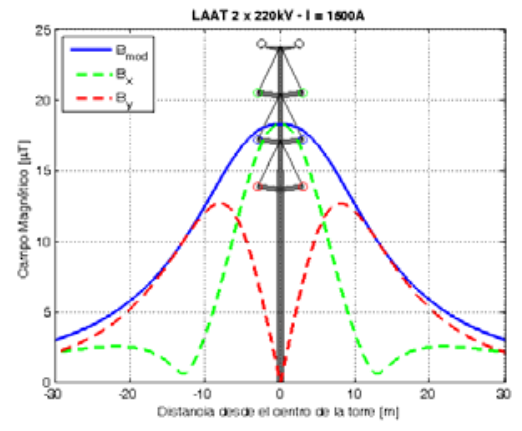

c) CV-LP.

Fig. 3 - Valores de Campo Magnético, generados por LAAT 2x220kV (I=1500A).

Las tres estructuras corresponden a tres generaciones constructivas, la primera pertenece a líneas instaladas en la década del '70, la franja de servidumbre que corresponde a esta es la más amplia de todas, debido a que la distancia entre los conductores externos es la mayor, aproximadamente $28 \mathrm{~m}$.

La segunda de las estructuras, que utiliza aisladores de suspensión y configuración coplanar vertical posee una distancia entre conductores externos de aproximadamente $8 \mathrm{~m}$, esto es 3 veces menor que la anterior. Esto hace que la estructura sea más compacta y los valores de campo sean inferiores.

Por último la estructura con aisladores tipo Line-Post, con una distancia entre conductores externos de aproximadamente $6 \mathrm{~m}$, es la más compacta de todas y consecuentemente los valores de campo magnético, para una misma condición de carga, son los más bajos, comparando las tres estructuras analizadas.

En general, puede decirse que las topologías más compactas son las que arrojan mejores resultados.

\subsection{Campo Magnético generado por Cables Subterráneos.}

Para caracterizar los valores de campo magnético generados por Cables Subterráneos de Alta Tensión (CSAT) hay que considerar dos zonas, la correspondiente a ductos, en la que los conductores de fase se encuentran próximos, pueden encontrarse en trébol o en configuración coplanar horizontal o vertical.

En la zona de empalmes se observa una mayor separación entre los conductores de fase, que varía de acuerdo al nivel de tensión. A medida que aumenta la tensión también los hacen la separación entre fases y consecuentemente los niveles de campo magnético. El incremento de la separación se debe fundamentalmente al espacio necesario para realizar los empalmes, 
esto puede apreciarse en la Fig. 4, en donde se presentan esquemas correspondientes a la zona de ductos a) y la zona de empalmes b).

\subsubsection{Campo Magnético generado por cables subterráneos simple terna}

En este ejemplo correspondiente a una terna de $220 \mathrm{kV}$, en la zona de ductos los conductores se disponen en trébol con una separación entre fases de $200 \mathrm{~mm}$. La profundidad desde el nivel del suelo es de aproximadamente $2 \mathrm{~m}$, considerando el centro de los conductores inferiores.

Si se considera ahora la zona de empalmes, se observa que el centro de los conductores se ubica a $1900 \mathrm{~mm}$ del nivel del suelo y la separación entre fases contiguas es de $500 \mathrm{~mm}$. La distancia entre fases externas es de $1000 \mathrm{~mm}$.

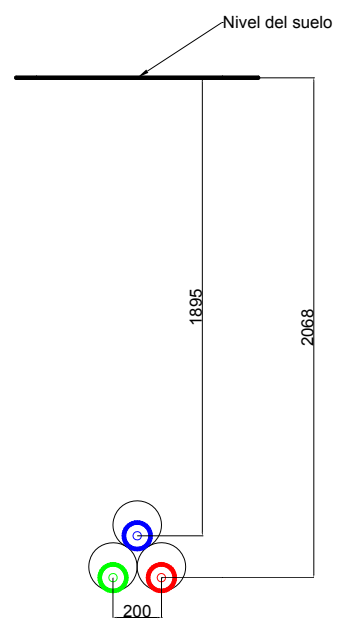

a) Disposición en zona de ductos

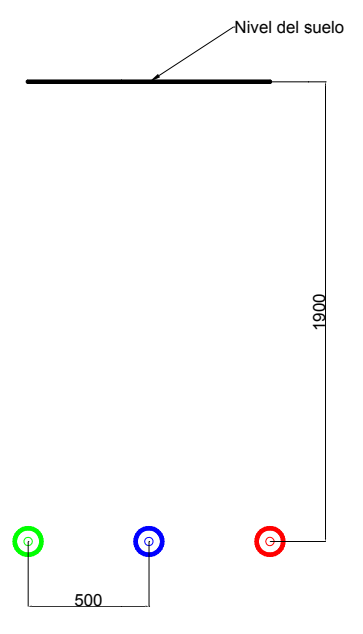

b) Disposición en fosa de empalmes

Fig. 4 - Ejemplo de disposición de conductores en zona de ductos y fosa de empalmes, CSAT $220 \mathrm{kV}$.

En la Fig. 5 se presenta una imagen de la fosa de empalmes, en la que se aprecian los tres empalmes realizados en una terna de $220 \mathrm{kV}$.

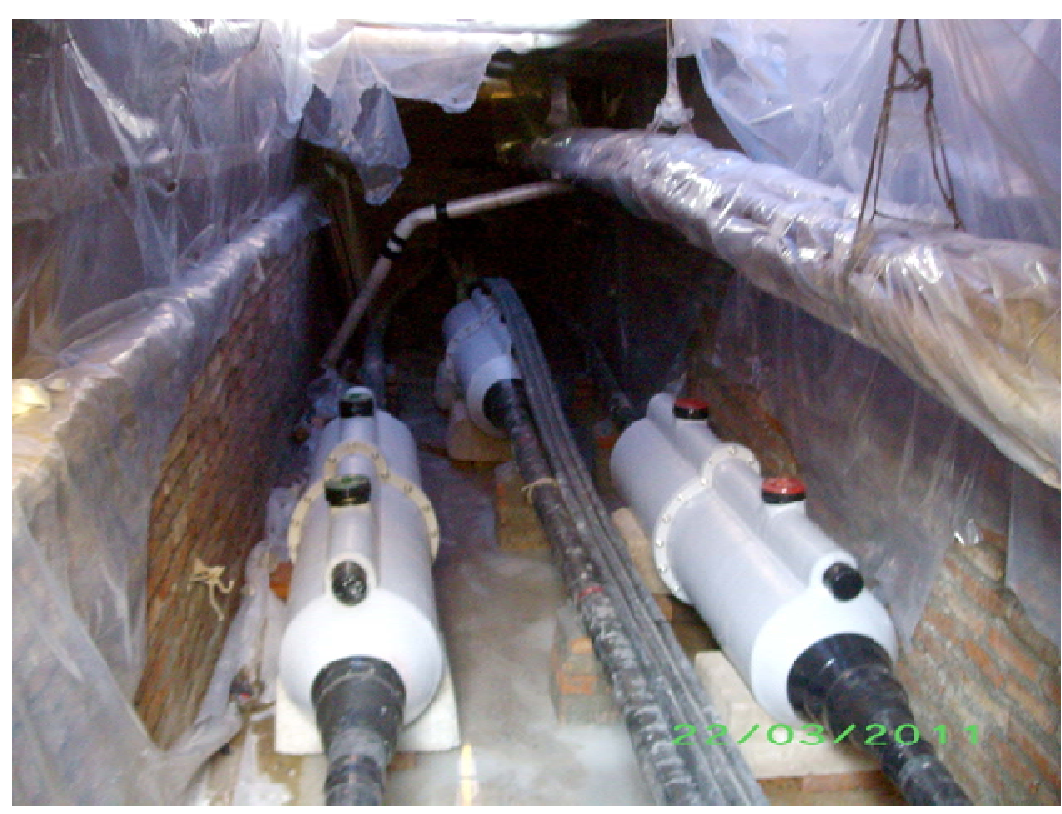

Fig. 5 - Fosa de empalmes, CSAT $220 \mathrm{kV}$. 
En la Fig. 6 se presentan los valores campo magnético, obtenidos mediante cálculos realizados a partir de un modelo tridimensional, en el que se representaron todos los conductores involucrados, considerando las posiciones adoptadas por estos en zona de ductos y zona de empalmes. Los valores corresponden a una corrientes de $790 \mathrm{~A}$.

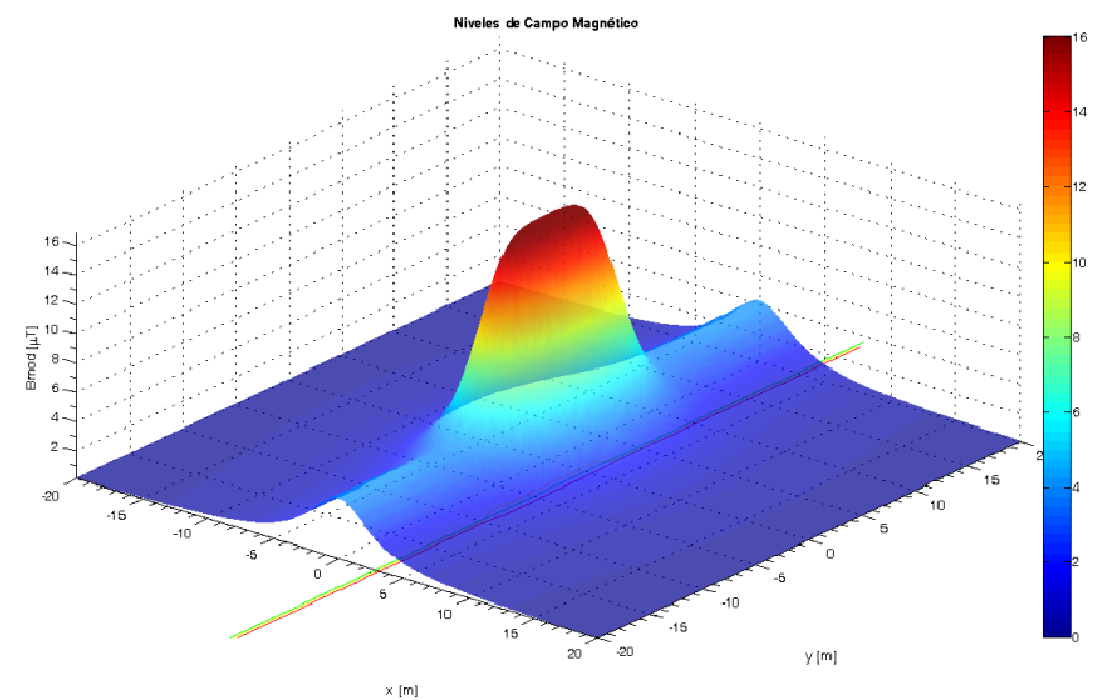

Fig. 6 - Campo magnético generado por CSAT $220 \mathrm{kV}$, en zona de ductos y fosa de empalmes.

Del análisis de la Fig. 6, se pueden identificar dos zonas, la de ductos en donde los valores de campo magnético máximo en la zona central es de aproximadamente $4 \mu \mathrm{T}$ y la zona de empalmes en donde los valores de campo son de aproximadamente $16 \mu \mathrm{T}$.

Para analizar con más detalles los valores obtenidos anteriormente, en la Fig. 7 se grafican perfiles trasversales, a la traza de los conductores, de campo magnético sobre la zona de ductos (azul) y sobre la zona de empalmes (rojo). Estos se obtuvieron por medio de herramientas de cálculo en 2D.

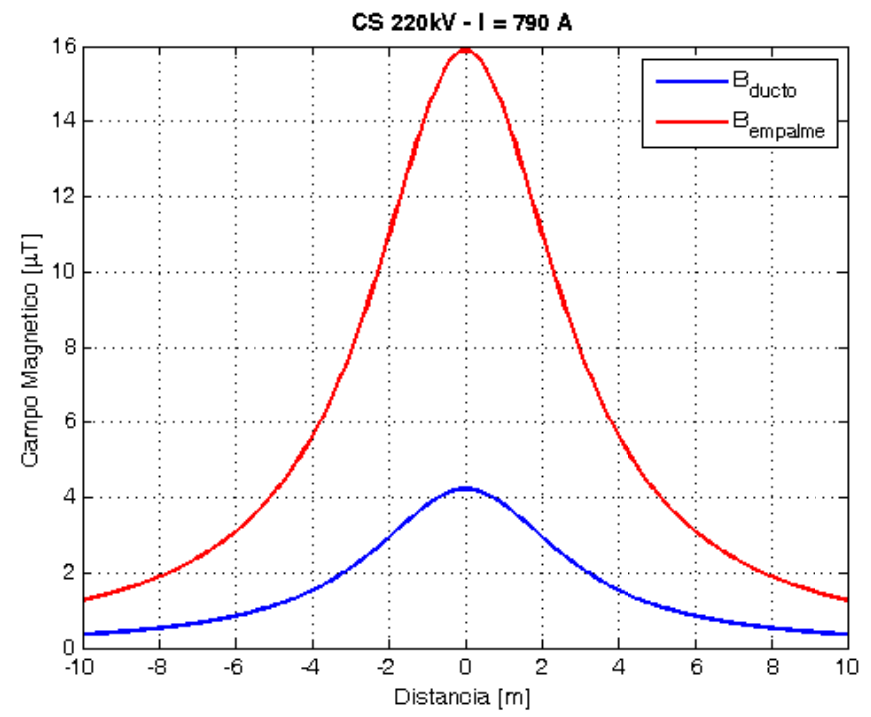

Fig. 7 - Perfiles de campo magnético generado por CSAT $220 \mathrm{kV}$, en zona de ductos y fosa de empalmes.

Se destaca el aumento de los valores de campo sobre la fosa de empalmes, en donde se triplican los valores de campo magnético para la configuración planteada en este ejemplo. 


\subsubsection{Campo Magnético generado por cables subterráneos con múltiples ternas}

Es común encontrar en el tendido de la traza de cables más de una terna. Este tipo de instalaciones es muy común a las salidas de las Estaciones Transformadoras, luego las ternas se separan para alimentar diferentes Subestaciones. Resulta de interés estudiar cuales son los valores de campo magnético generado, considerando tanto las zonas de ductos y fosa de empalmes.

Se propone analizar un ejemplo en el que se considera una doble terna de cable subterráneo de $132 \mathrm{kV}$. En las Fig. 8 a) y b) se presentan cortes transversales de las zonas mencionadas, en las mismas también se aprecian con diferentes colores las secuencias de energización de las ternas, R: verde, S: azul y T: rojo.

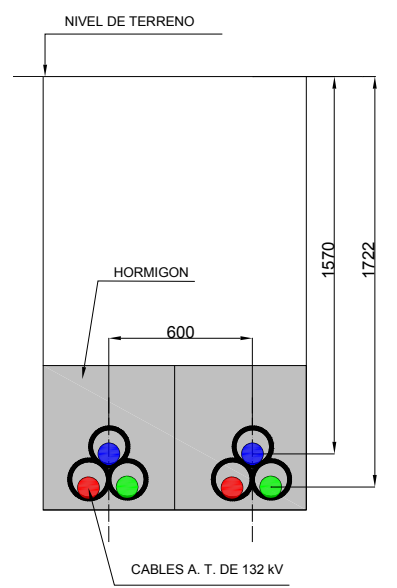

a) $\quad \operatorname{CSAT} 2 \times 132 \mathrm{kV}$, zona de ductos

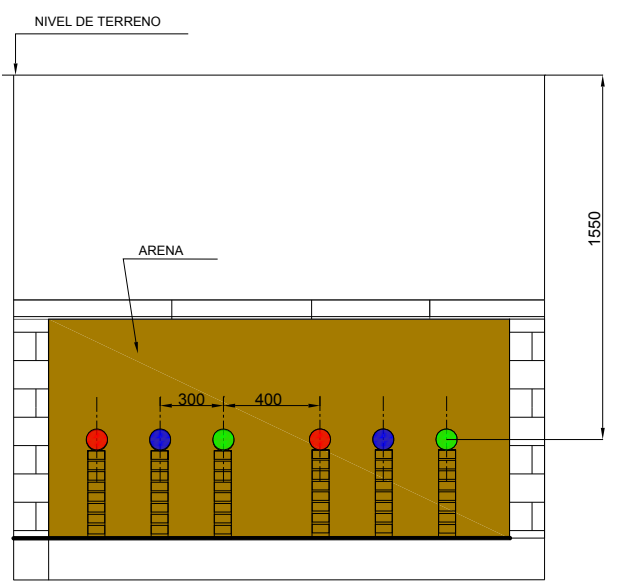

b) CSAT $2 \times 132 \mathrm{kV}$, zona de empalmes

Fig. 8 - Ejemplo de disposición de conductores en zona de ductos y fosa de empalmes, CSAT $2 \times 132 \mathrm{kV}$.

Los valores de campo generados se presentan en las Fig. 9 y Fig. 10, se observa el incremento en los valores de campo magnético en la zona correspondiente a los empalmes. Un detalle de esto se aprecia en la Fig. 10, en donde se trazan los perfiles correspondientes a las zonas de ductos y fosa de empalmes.

Los mayores valores obtenidos son de $11,3 \mu \mathrm{T}$ y $28 \mu \mathrm{T}$, en la zona de ductos y fosa respectivamente, para una corriente de $1010 \mathrm{~A}$. Se destaca que en la zona de empalmes se supera el valor máximo permitido por la reglamentación vigente en la República Argentina $(25 \mu \mathrm{T})$.

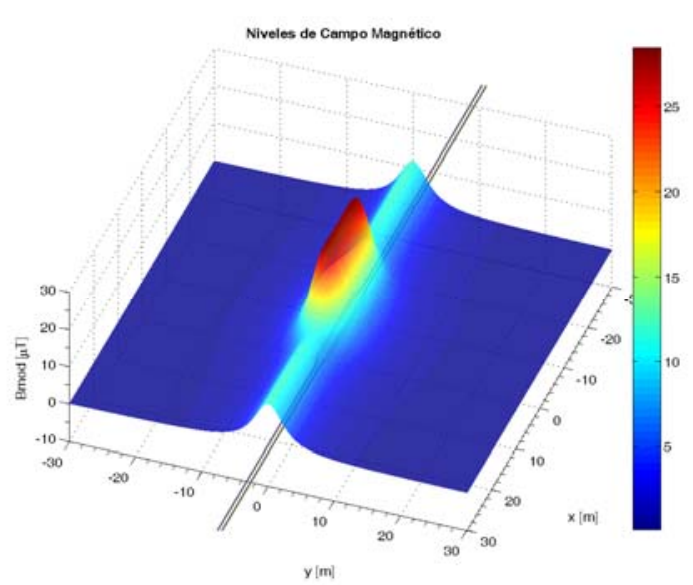

Fig. 9 - Mapa de niveles de B, CSAT doble terna.

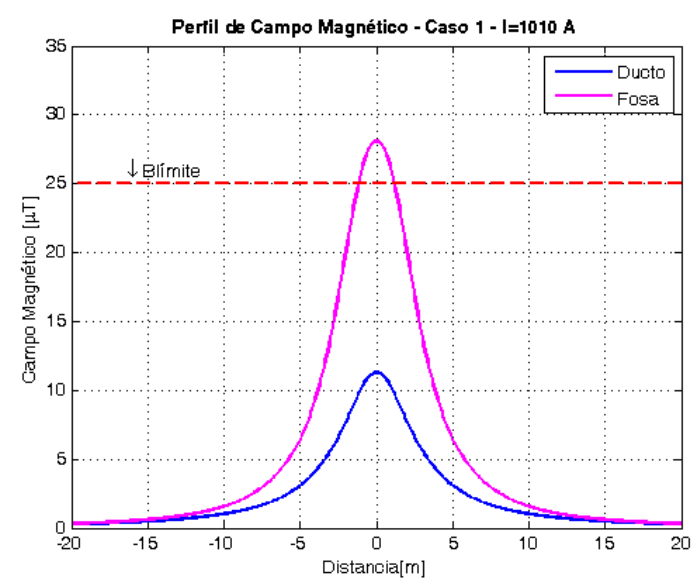

Fig. 10 - Perfiles Transversales, CSAT doble terna. 


\subsection{Campo Magnético generado por Estaciones Transformadoras}

A medida que las LAAT se acercan a los grandes centros urbanos es necesario reducir los niveles de tensión. Esta reducción se realiza en estaciones o subestaciones transformadoras, que pueden poseer dos o más niveles de tensión. Para el cálculo del campo magnético generado por este tipo de instalaciones es necesario realizar modelos tridimensionales, que permitan reproducir la traza de los diferentes conductores que acometen y recorren internamente la estación.

En este punto se presenta un ejemplo de una Estación Transformadora (ET) que cuenta con 2 transformadores $300 \mathrm{MVA}$, alimentados en $220 \mathrm{kV}$, con salidas en $132 \mathrm{kV}$ en la parte de alta tensión. Dichos transformadores se alimentan a través de un cable subterráneo y una línea aérea.

Además, la ET cuenta con 3 transformadores de 40 MVA alimentados en $132 \mathrm{kV}$, con salidas en 13,2 kV. Dichos transformadores se encuentran en una barra de $132 \mathrm{kV}$ junto con otros 8 cables subterráneos provenientes de diferentes subestaciones. Las salidas en media tensión se realizan mediante 27 cables subterráneos.

En la Fig. 11 se aprecia una imagen en donde se superponen el layout de las instalaciones modeladas y los valores de campo magnético. En trazo negro grueso se marca el perímetro de las instalaciones. Sombreado en gris se indica la ubicación de los transformadores. En color verde se marcan las instalaciones en $220 \mathrm{kV}$ (líneas aéreas, cables subterráneos y barras). En color índigo, se indican las instalaciones en $132 \mathrm{kV}$ y en rojo las instalaciones en $13,2 \mathrm{kV}$. Los valores de campo magnético en el perímetro, cada color representa un valor de campo magnético, de acuerdo a la referencia de barra de la derecha.

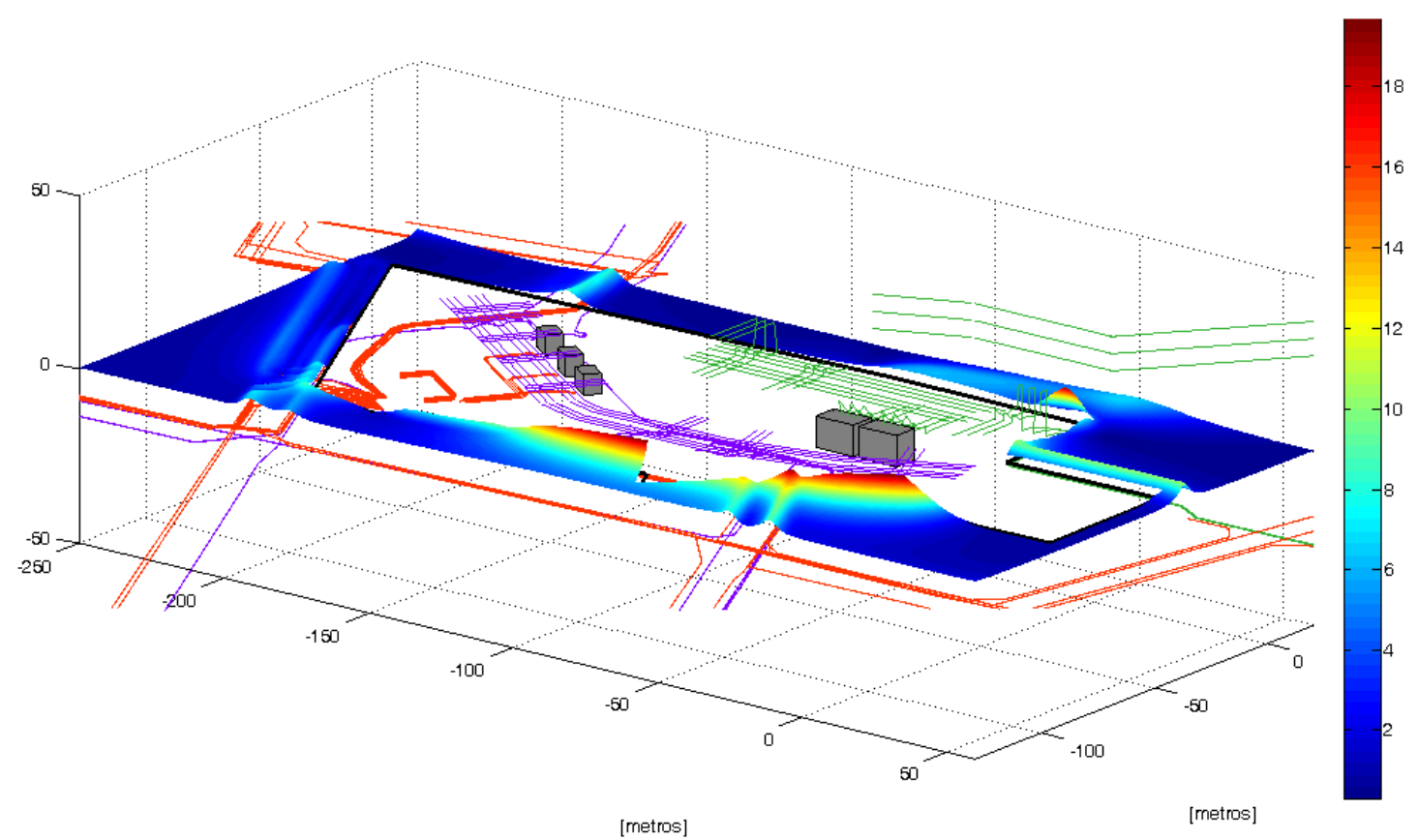

Fig. 11 - Campo magnético generado por una subestación transformadora.

Los niveles de campo varían de acuerdo a la carga de los alimentadores y el desbalance de corrientes existente. Se observan los mayores valores de campo en zonas de acometidas de conductores o bien en aquellas zonas en donde los conductores se encuentran más próximos a la zona de cálculo. 


\subsection{Campo Magnético generado por Centros de Transformación.}

Los Centros de Transformación (CT) constituyen la última etapa en el proceso de distribución de la energía eléctrica, estos reducen el nivel de tensión de Media Tensión (MT) a Baja Tensión (BT). Estas instalaciones se las puede encontrar en diferentes tipos constructivos, aéreos, subterráneos o a nivel. A diferencia de las instalaciones consideradas hasta ahora, en estas se observa la presencia de un conductor de neutro y en la mayoría de los casos existe corriente por el mismo ya que las cargas suele ser desequilibradas.

Para el cálculo de estas instalaciones, al igual que las ET, es necesario realizar modelos tridimensionales.

En la Fig. 12 se presenta una instalación típica de un CT a nivel, con el layout de la instalación. En el mismo se representan los conductores de MT por los que se realiza la acometida al CT y las Celdas de MT. El conductor en MT que vincula las Celdas y el Transformador, en este caso de $800 \mathrm{kVA}$. Del lado de BT se observan, en este caso las barras, que vinculan el transformador y el tablero de distribución de BT. También y los conductores de BT con los que se extrae la potencia, en este caso se consideran 8 alimentadores.

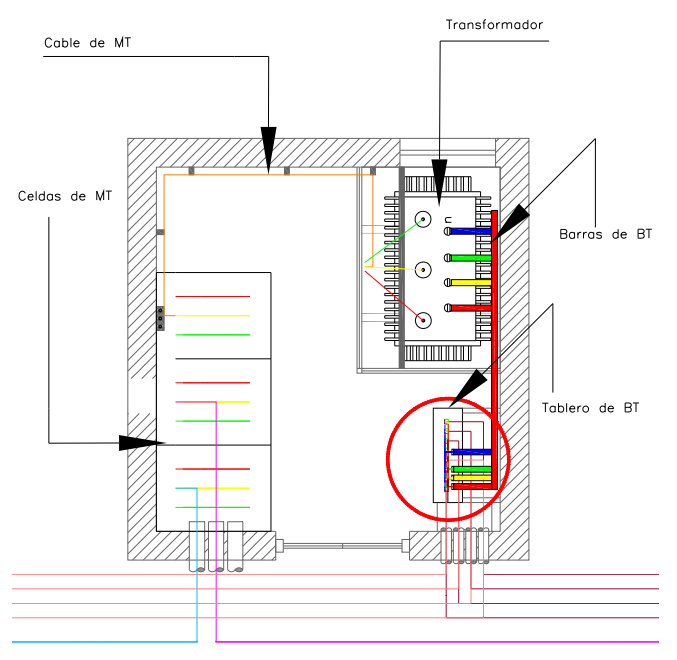

Fig. 12 - Layout del CT considerado.

En las Fig. 13 y Fig. 14 se presentan los niveles de campo magnético generados, considerando una condición de carga equilibrada, se considera una corriente de 490 A por las barras de BT. Esta corriente se distribuye por lo ocho alimentadores de BT. En estas se superponen el layout de las instalaciones modeladas y los valores de campo magnético. En trazo negro y líneas punteadas se marca el perímetro de las instalaciones. Sombreado en gris se indica la ubicación del transformador. En color negro continuo se marcan los conductores en 13,2 kV y los conductores de $0,38 \mathrm{kV}$. Los valores de campo magnético en el perímetro, cada color representa un valor de campo magnético, de acuerdo a la referencia de barra de la derecha.

En la Fig. 13 se presenta un mapa con los valores de campo en el perímetro del CT. En la Fig. 14 se vuelcan los mismos resultados, pero a través de curvas de igual nivel de campo magnético. 


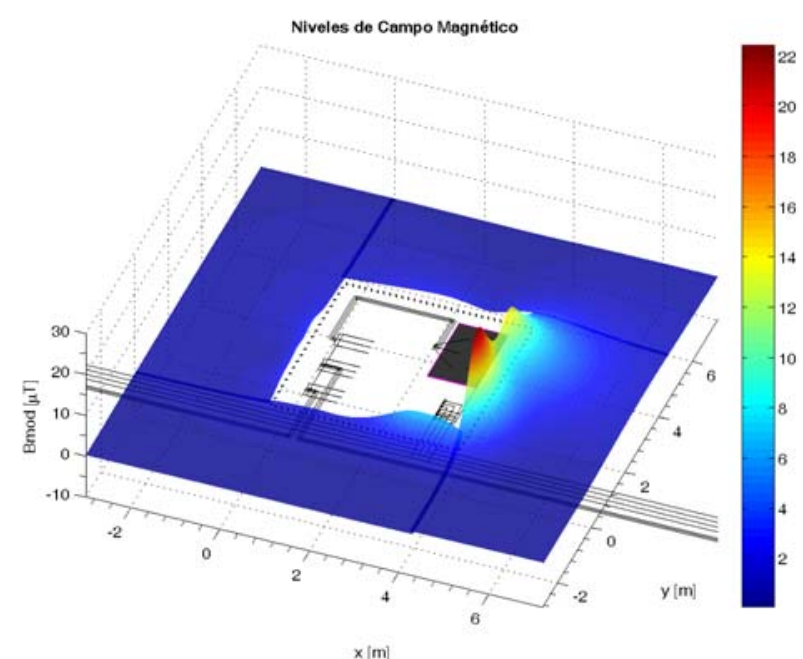

Fig. 13 - Mapas de B, en un CT.

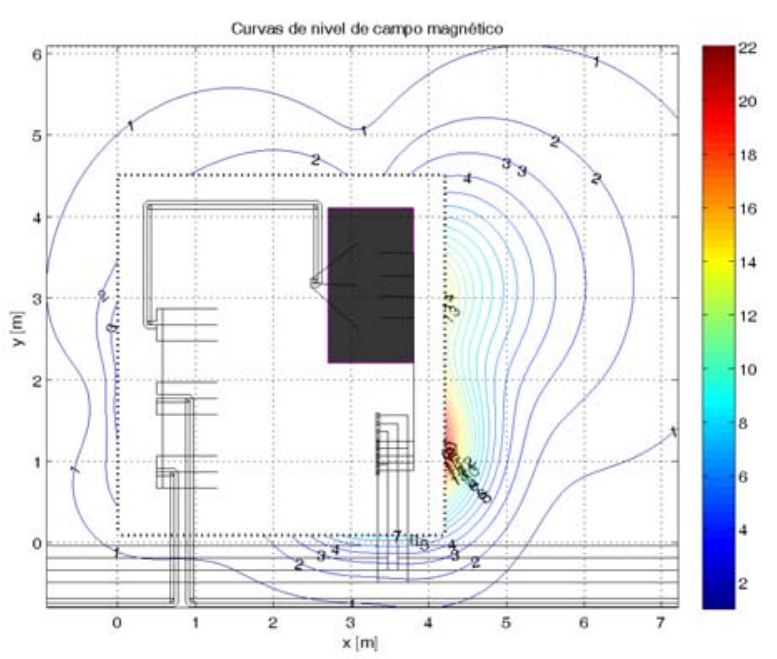

Fig. 14 - Curvas de nivel de B, en un CT.

En este caso se observa que la zona en donde se disponen los conductores de baja tensión y el tablero de distribución, es la zona en donde se presentan los mayores valores de campo magnético. Esto se debe a que allí se encuentran las mayores corrientes, fuentes de campo magnético.

\subsection{Comparación de mediciones}

En este punto se comparan los resultados obtenidos por medio de cálculos y mediciones para LAAT. Para estos casos se consideraron las cargas al momento de realizar las mediciones. Se indican además la secuencia de energización de las fases (R: verde, S: azul y T: rojo). En el Anexo VI, se indican algunas consideraciones sobre medición de campo magnético.

En la Fig. 15 se presenta la comparación entre mediciones y simulaciones para una LAAT 2 x $220 \mathrm{kV}$, configuración CH-AS, la carga de la línea al momento de la medición fue de $200 \mathrm{~A}$.

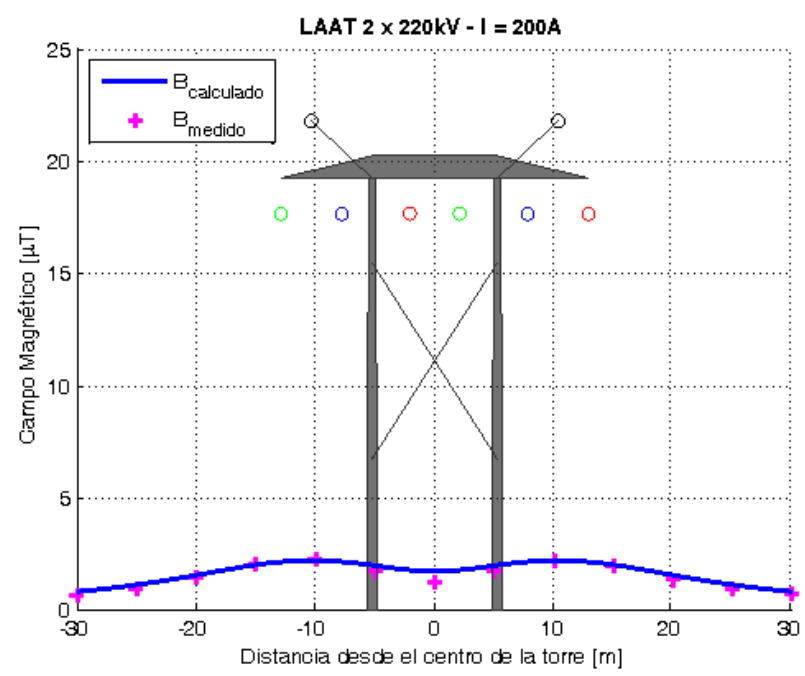

Fig. 15 - Valores de campo B medidos y calculados, LAAT 2 x $220 \mathrm{kV} \mathrm{CH-AS,} \mathrm{I=200} \mathrm{A.}$

En la Fig. 16 se presenta la comparación entre mediciones y simulaciones para una LAAT 2 x $220 \mathrm{kV}$, configuración CV-AS, la carga de la línea al momento de la medición fue de $1300 \mathrm{~A}$. 


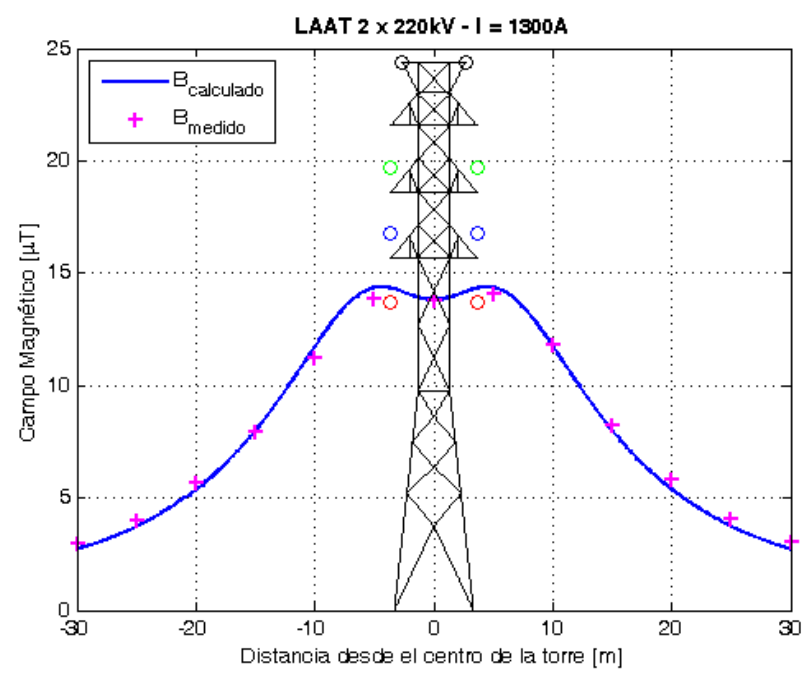

Fig. 16 - Valores de campo B medidos y calculados, LAAT 2 x $220 \mathrm{kV}$ CV-AS, I=1300 A.

En la Fig. 17 se presenta la comparación entre mediciones y simulaciones para una LAAT 2 x $220 \mathrm{kV}$, configuración CV-LP, la carga de la línea al momento de la medición fue de $650 \mathrm{~A}$.

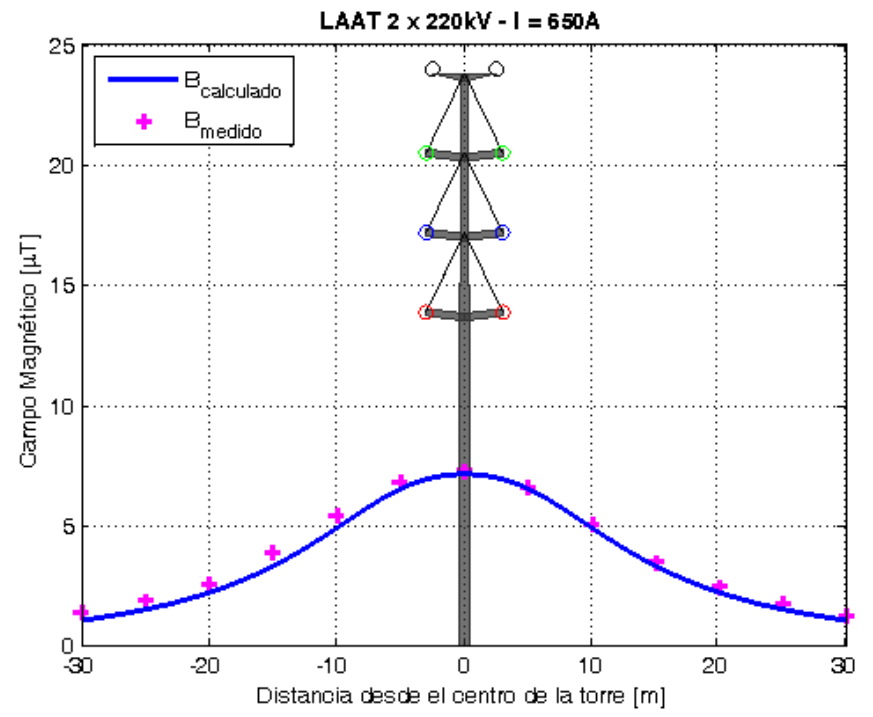

Fig. 17 - Valores de campo B medidos y calculados, LAAT 2 x 220 kV CV-LP, I=650 A.

Para los cálculos se contemplaron modelos bidimensionales. Se evaluaron diferentes tipos de líneas, con diferentes condiciones de carga. Los resultados obtenidos son satisfactorios ya que los perfiles obtenidos por cálculo presentan una buena aproximación a los obtenidos en las mediciones.

En algunos casos se observan algunas diferencias, estas pueden deberse principalmente a dos causas, posibles irregularidades en el terreno bajo la línea y posibles desbalances en las corrientes de las diferentes fase, no contempladas en los modelos. 


\section{Técnicas de Mitigación}

Para reducir los niveles de campo magnético existen diferentes técnicas que permiten lograr el objetivo, entre las que se puede destacar el manejo de los conductores, la utilización de pantallas y compensación.

En esta sección se describen los principios generales utilizados y algunos ejemplos de mitigación, presentando simulaciones y casos prácticos implementados en laboratorio.

\subsection{Incremento de la distancia a la fuente de campo magnético}

A medida que el punto de interés se aleja de la fuente de campo magnético disminuyen los valores de campo magnético. Para el caso objeto de este trabajo las fuentes las constituyen los conductores de fase. En general puede decirse que si se analiza un sistema con corrientes balanceadas, el campo magnético decae con la inversa del cuadrado de la distancia, si lo que se tiene es un sistema desbalanceado, en general la caída se da con la inversa de la distancia.

Para ejemplificar esta situación en la Fig. 18, se aprecia una terna de conductores con una corriente de fase de $2000 \mathrm{~A}$, en el entorno de la misma se presentan las curvas de nivel de campo magnético. Se proponen dos puntos de interés, P1 y P2, alejados de la fuente de campo 20 y $40 \mathrm{~m}$ respectivamente.

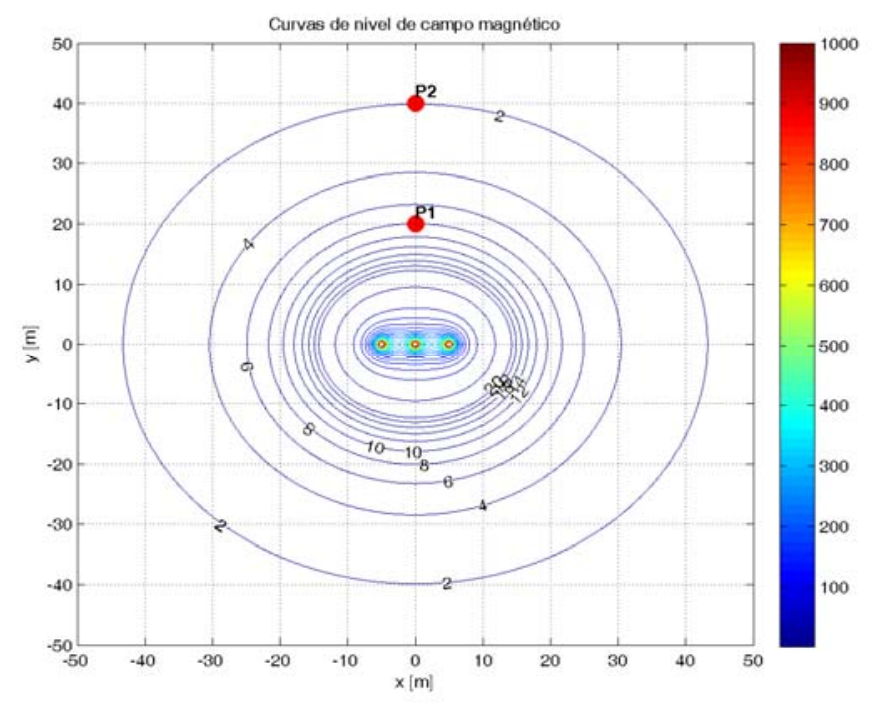

Fig. 18 - Valores de campo $B[\mu \mathrm{T}]$, para diferentes distancias a la fuente, $\mathrm{I}=2000 \mathrm{~A}$.

Puede observarse que a medida que el punto de interés se encuentre más lejos de la fuente menores serán los valores de campo, para punto P1 situado a $20 \mathrm{~m}$ de la fuente el valor de campo B es de $8 \mu \mathrm{T}$, mientras que en el punto P2 situado a $40 \mathrm{~m}$ de la fuente el valores correspondiente de campo es de $2 \mu \mathrm{T}$.

Se destaca que el alejamiento de las fuentes es una de las alternativas básicas para lograr mitigar el campo magnético. Cuanto más alejado este el punto de interés de los conductores de fase, menores serán los valores de campo magnético.

\subsection{Ubicación de conductores}

Esta técnica es aplicable a sistemas eléctricos que cuenten con al menos dos conductores, el agrupamiento de los conductores de las instalaciones eléctricas influye en los valores de campo magnético, en el entorno en el cual estos se hallan inmersos, es por eso que de acuerdo a las distancias entre los mismos y su posición relativa se obtendrán diferentes niveles de campo. 
Como ejemplo de esto se pueden tomar como referencia dos tipos de estructuras utilizadas en la República Argentina para líneas de transmisión de $500 \mathrm{kV}$, en primer lugar se considera un diseño típico conocido como torre "V-Arriostrada" (VA) y por otro lado el diseño compacto “Cross Rope" (CR), ver Fig. 19 y Fig. 20 respectivamente.

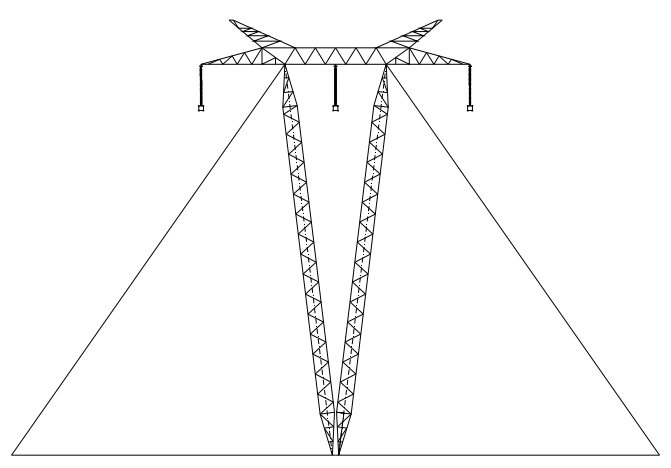

Fig. 19 - Torre V-Arriostrada $500 \mathrm{kV}$

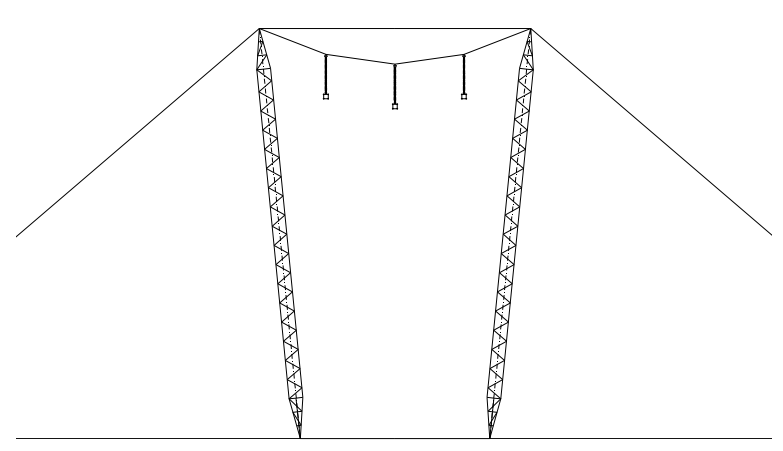

Fig. 20 - Torre Cross Rope $500 \mathrm{kV}$

En la Tabla IV se presentan las principales características de estos dos tipos de estructuras. Se destaca una diferencia importante en la distancia entre conductores externos, en el caso de la VA esta distancia es de $26 \mathrm{~m}$ y en el caso de la CR es de $14 \mathrm{~m}$.

Tabla IV - Características constructivas de torres para LAAT de $500 \mathrm{kV}$

\begin{tabular}{|c|c|c|}
\hline \multirow{2}{*}{ Dimensión } & \multicolumn{2}{|c|}{ Torre } \\
\cline { 2 - 3 } & V-Arriostrada & Cross Rope \\
\hline Altura de conductores en torre [m] & 33,5 & 33,5 \\
\hline Altura de conductores en el vano [m] & 10,8 & 10,8 \\
\hline Altura de torre [m] & 42,3 & 41,5 \\
\hline Distancia entre conductores externos [m] & 26 & 14 \\
\hline Ancho de franja de servidumbre [m] & 79 & 67 \\
\hline
\end{tabular}

En las Fig. 21 y Fig. 22, se presentan los perfiles transversales de campo magnético para los dos tipos de LAAT considerados en este punto. Analizando los resultados obtenidos, se destaca que la configuración más compacta, con menor distancia entre conductores es la que arroja menores valores de campo magnético.

En el caso de la VA el valor máximo obtenido es de 25,5 $\mu \mathrm{T}$ en el centro de la torre. Para el caso de la CR el valore el valor máximo es de 20,6 $\mu \mathrm{T}$. Considerando una corriente de $1500 \mathrm{~A}$ por los conductores. 


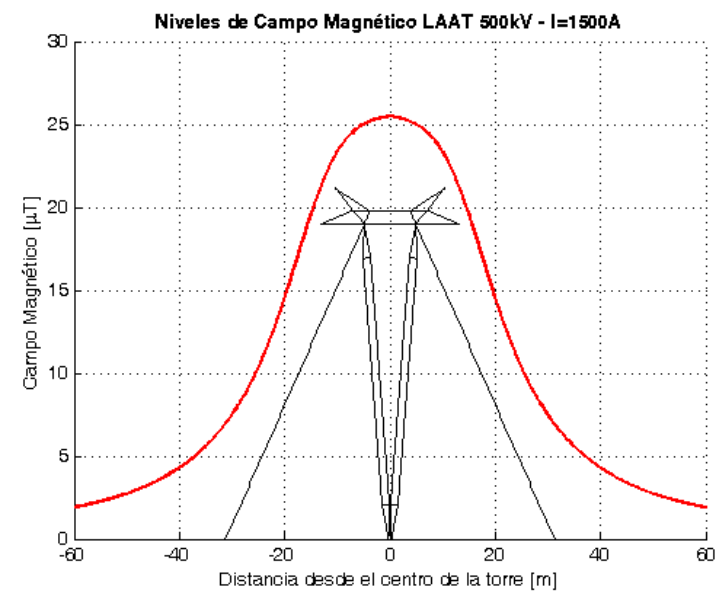

Fig. 21 - Valores de campo magnético en LAAT $500 \mathrm{kV}$ VA, I=1500 A.

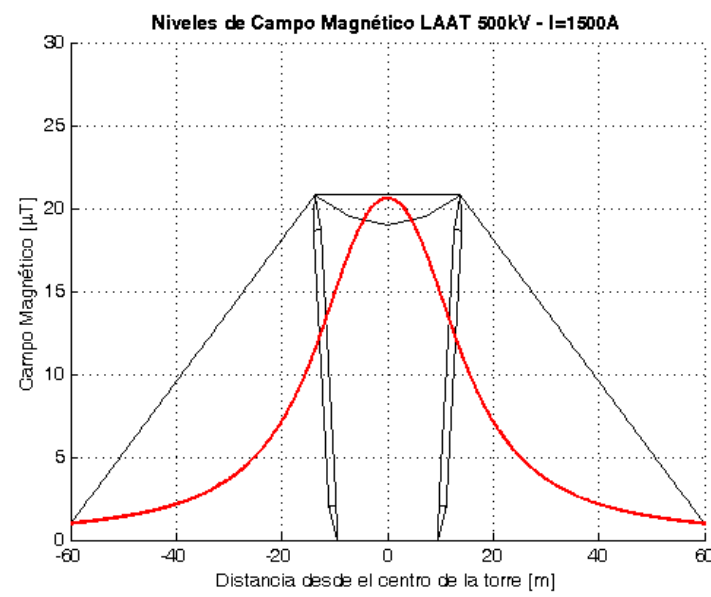

Fig. 22 - Valores de campo magnético en LAAT $500 \mathrm{kV}$ CR, I=1500 A.

Además el perfil en el caso de la CR presenta en toda su extensión menores valores de campo magnético.

En general se puede decir que las configuraciones más compactas ofrecen mejores resultados desde el punto de vista de los valores de campo magnético.

En el Anexo VII se presenta un análisis de sensibilidad de los valores de campo magnético para diferentes configuraciones de conductores de fase. Se consideran allí corrientes equilibradas y desequilibradas.

\subsection{Cambio de secuencia de energización}

Esta técnica de mitigación de campo magnético es aplicable a circuitos que cuentan con más de una terna, básicamente consiste en cambiar la posición relativa de las fases para circuitos diferentes, en otros términos se produce un cambio en la secuencia de energización de las ternas. En el punto AVII-2 del Anexo VII, se presenta un análisis de sensibilidad a este tipo de solución.

En este punto se presentan algunos ejemplos en los que se consideran LAAT y CSAT.

Para la comparación entre las diferentes posibilidades se utilizará el Factor de Apantallamiento FA definido en la Ec. 1. Este factor indica en grado de reducción de campo magnético considerando una alternativa como referencia.

\subsubsection{Ejemplos de cambios de secuencias de energización en LAAT -Doble Terna}

Se consideran LAAT doble terna en $220 \mathrm{kV}$, las cuales ya fueron presentadas anteriormente en el inciso 5.2. En este punto se estudian alternativas de energización de las dobles ternas.

\subsubsection{Resultados LAAT 2x220 kV-CH-AS}

En primer lugar se presentan los resultados obtenidos para la topología CH-AS, presentada en la Fig. 2 a). Se consideraron dos secuencias de energización. En la Fig. 23 se presentan los perfiles de campo magnético obtenidos. 


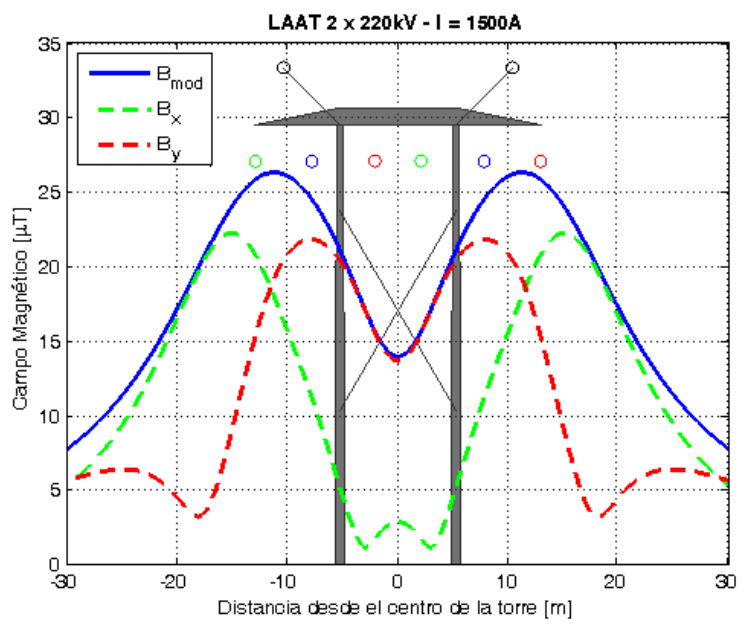

a) Secuencia de energización RST-RST (CH-AS a).

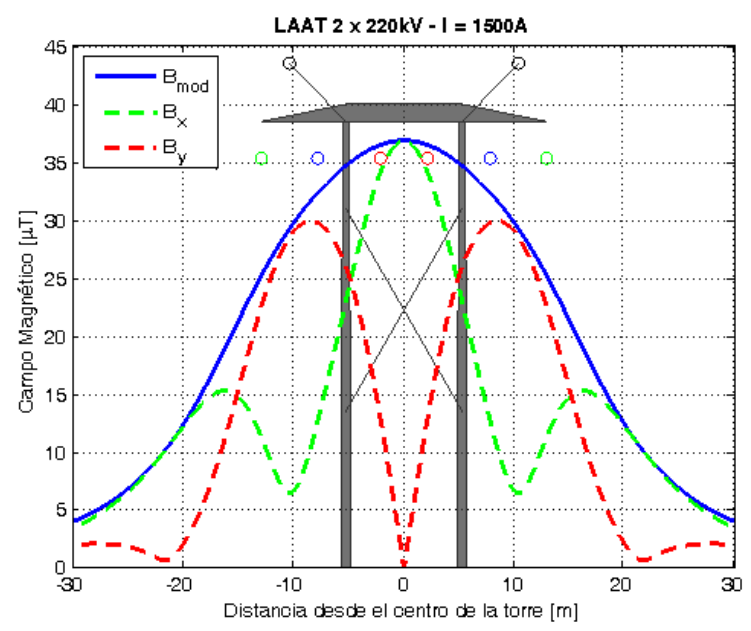

b) Secuencia de energización RST-TSR (CH-AS b).

Fig. 23 - Valores de campo B calculados, LAAT 2 x $220 \mathrm{kV}$ coplanar horizontal (fase R: verde, fase S: Azul y fase T: rojo), I=1500 A.

En la Fig. 24 a) se presenta una comparación de los valores de campo magnético (Bmod). En la Fig. 24 b) se presenta el perfil para FA, se toma como referencia la secuencia de energización correspondiente al CH-AS a.

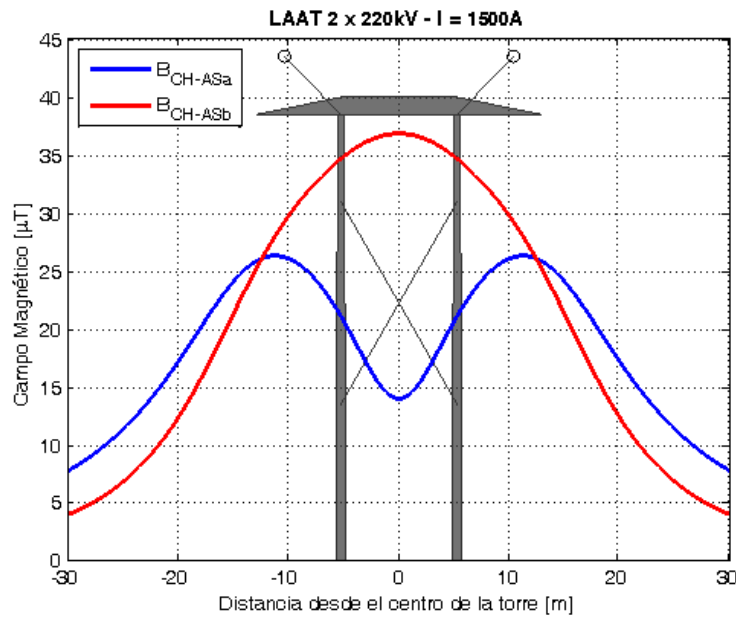

a) Valores de B, con secuencia de energización RST-RST y RST-TSR

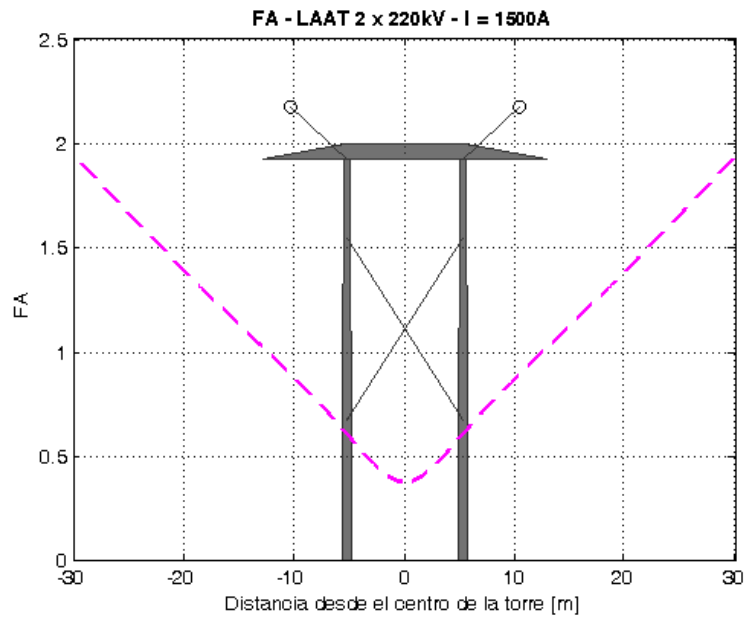

b) FA, correspondiente a las secuencias de energización RST-RST/ RST-TSR

Fig. 24 - Valores de campo B y FA, LAAT 2 x $220 \mathrm{kV}$ coplanar horizontal, I=1500 A.

Analizando los valores obtenidos, se destacan dos situaciones. En la parte exterior de los perfiles, la secuencia de energización identificada como $\mathrm{CH}-\mathrm{ASb}$, arroja menores valores de campo magnético, Fig. 24 a) perfil en color rojo. En la parte central ocurre lo contrario, se observa que este perfil arroja los mayores valores de campo.

Esta situación también se ve reflejada en la gráfica del FA, Fig. 24 b), considerando que cuando el $\mathrm{FA}=1$ los dos casos analizados arrojan igual valor de campo magnético, por lo que en la zona exterior los valores obtenidos arrojan valores mayores que 1 , llegando a 2 a $30 \mathrm{~m}$ desde el centro de la torre; esto significa que a esta distancia la alternativa b) reduce a la mitad los valores de campo magnético arrojados por la alternativa a). Sin embargo en la zona central los valores del FA son menores que 1, lo que significa que allí la alternativa a) en lugar de reducir los niveles de campo magnético, estos se incrementan. 
Para decidir cuál es la alternativa más favorable, en primer lugar hay que definir cuál es la zona en donde se pretende reducir los valores de campo magnético. Si se pretende obtener menores valores de campo magnético en la zona alejada del centro de la torre, la alternativa b) es la que arroja mejores resultados. Sin embargo, si lo que se quiere es reducir los valores en la zona central de la línea, la alternativa a) es la que arroja el mejor desempeño.

\subsubsection{Resultados LAAT 2x220 kV - CV-AS}

Para la topología presentada en la Fig. 2 b), CV-AS, con una de corriente de 1500 A, se obtienen los perfiles de campo magnético que se presentan en la Fig. 25, donde además se indican las secuencias de energización consideradas.

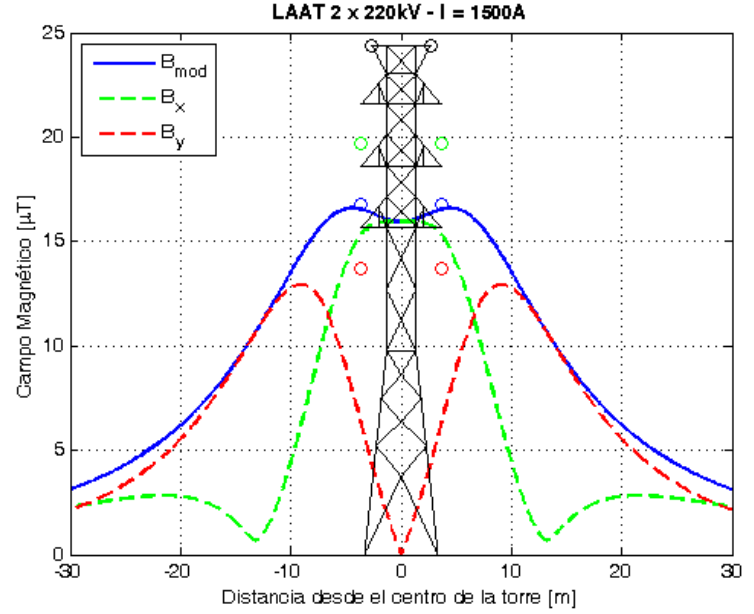

a) Secuencia de energización RST-RST (CV-AS a).

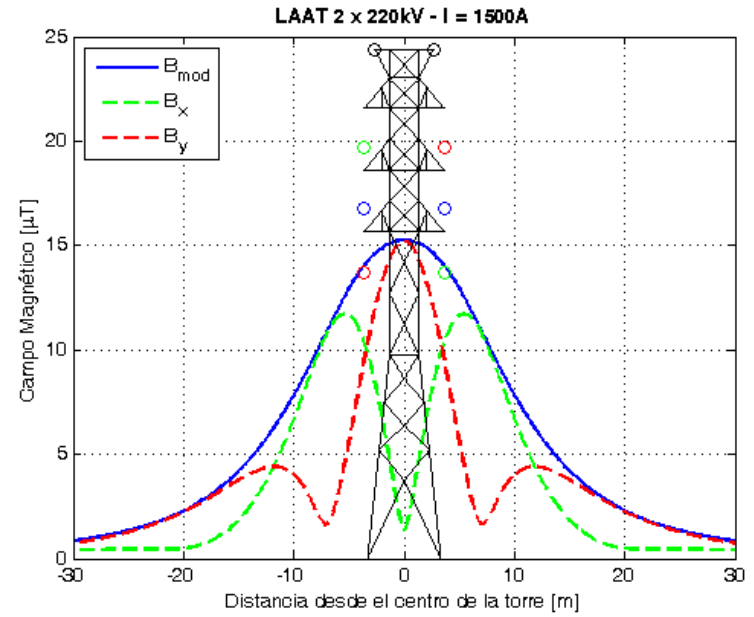

b) Secuencia de energización RST-TSR (CV-AS b).

Fig. 25 - Valores de campo B calculados, LAAT 2 x $220 \mathrm{kV}$ coplanar vertical (fase R: verde, fase S: Azul y fase T: rojo), I=1500 A.

En la Fig. 26 a) se presenta una comparación de los valores de campo magnético, calculados considerando las dos secuencias de energización. En la Fig. 26 b) se presenta el FA, se toma como referencia la secuencia de energización CV-ASa.

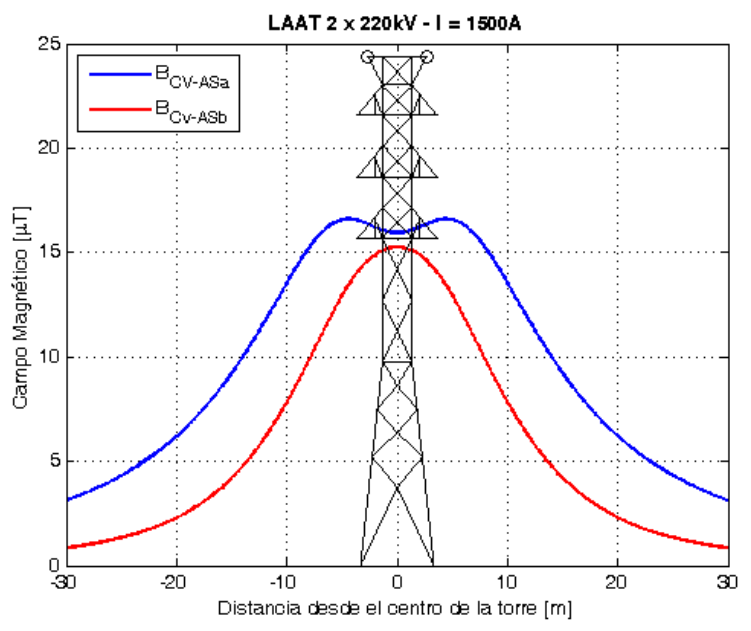

a) Valores de $B$, con secuencia de energización RST-RST y RST-TSR

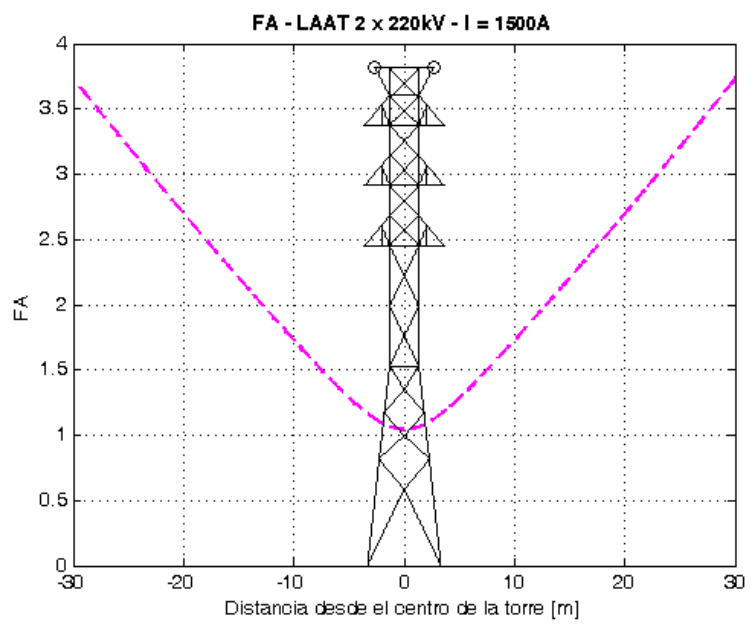

b) FA, correspondiente a las secuencias de energización RST-RST/ RST-TSR

Fig. 26 - Valores de campo B y FA, LAAT 2 x $220 \mathrm{kV}$ coplanar vertical, I=1500 A. 
En este caso, la configuración sin compensación de fases (CV-ASa) es la que arroja mayores valores de campo magnético, a lo largo de todo el perfil. Esto se aprecia en la Fig. 26 a) perfil azul. Esta situación también se ve reflejada en la gráfica del FA, Fig. 26 b), en donde se observa que a lo largo de todo el perfil el FA es mayor a 1. Los valores más altos se obtienen en los extremos $(F A=3,75)$.

La alternativa más favorable, para esta topología es la identificada como CV-AS b, con las fases compensadas, ya que a lo largo de todo el perfil es la que arroja menores valores de campo magnético.

\subsubsection{Resultados LAAT 2x220 kV - CV-LP}

A continuación se presentan los resultados obtenidos para la topología CV-LP, presentada en la Fig. 2 c), considerando una carga de 1500 A y diferentes secuencias de energización. En la Fig. 27 se presentan los resultados.

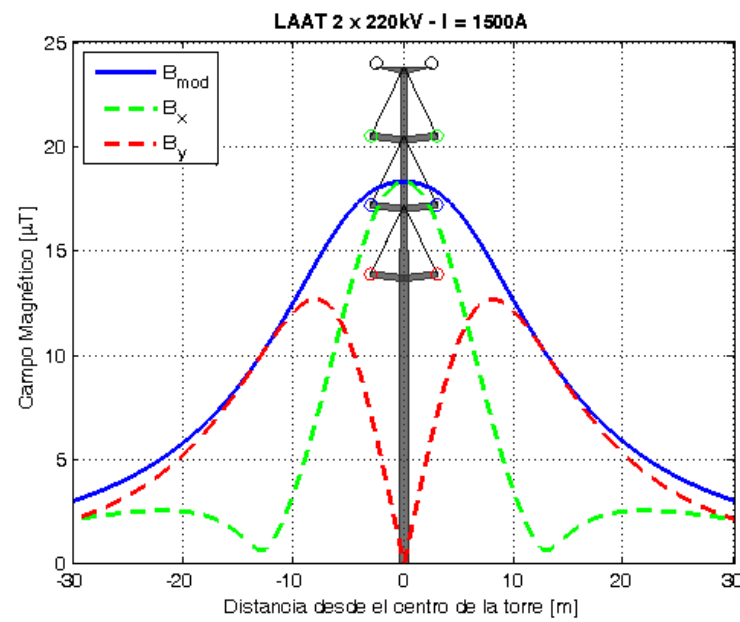

a) Secuencia de energización RST-RST (CV-LP a).

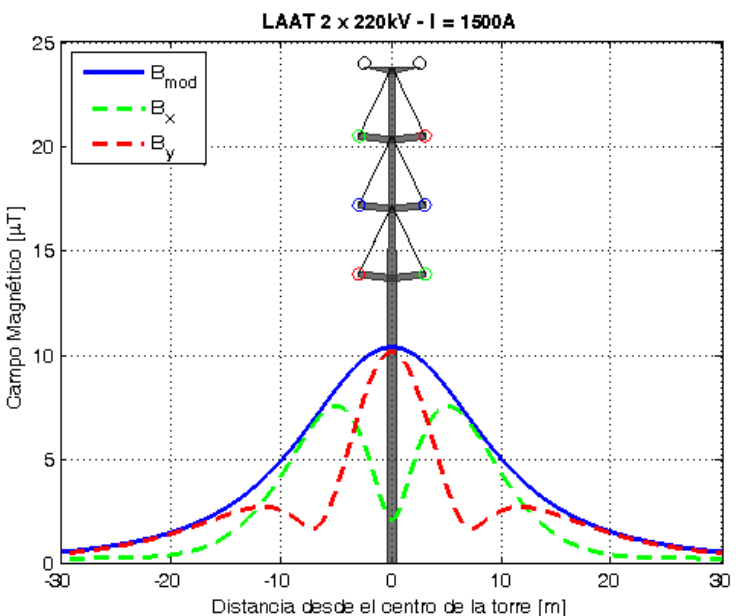

b) Secuencia de energización RST-TSR (CV-LP b).

Fig. 27 - Valores de campo B calculados, LAAT 2 x $220 \mathrm{kV}$ Coplanar vertical (fase R: verde, fase S: Azul y fase T: rojo), I=1500 A.

En la Fig. 28 a) se presenta una comparación de los valores de campo magnético, calculados considerando las dos secuencias de energización. En la Fig. 28 b) se presenta el perfil del FA, se toma como referencia la secuencia la secuencia de energización CV-LP a.

Comparando los valores obtenidos para esta topología, se obtienen también menores valores de campo en el caso en el que existe compensación de fases. Los valores para el caso CV-LPa son mayores que los obtenidos para el CV-LPb, Fig. 28 a) perfil azul y rojo respectivamente. Esta situación también se ve reflejada en la gráfica del FA, Fig. 28 b). 


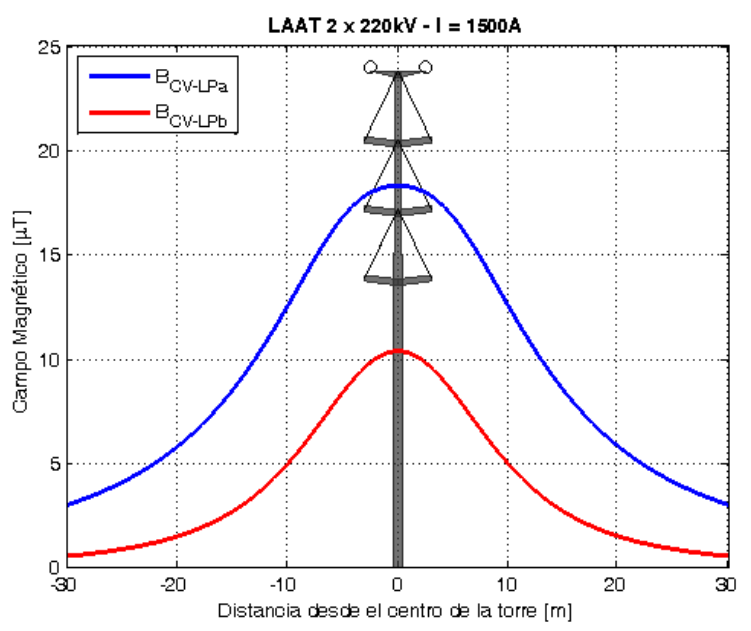

a) Valores de B, con secuencia de energización RST-RST y RST-TSR

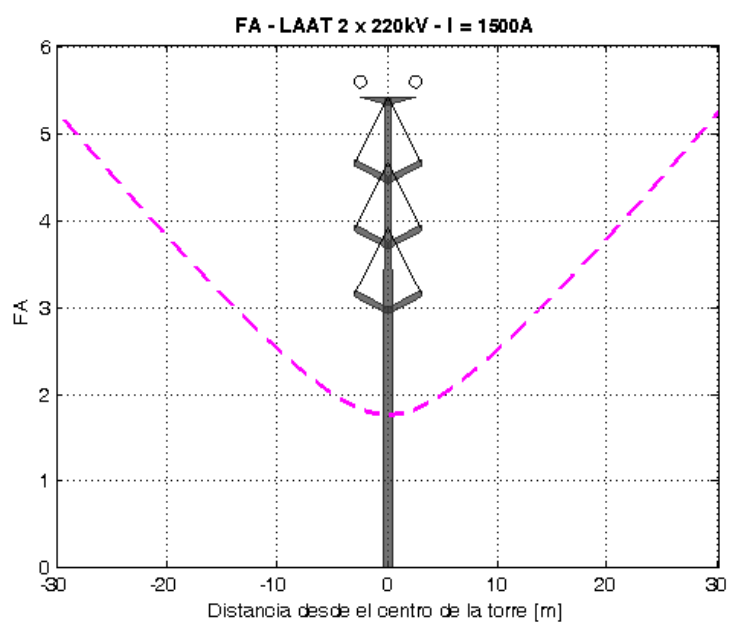

b) FA, correspondiente a las secuencias de energización RST-RST/ RST-TSR

Fig. 28 - Valores de campo B y FA, LAAT 2 x $220 \mathrm{kV}$ coplanar horizontal, I=1500 A.

\subsubsection{Ejemplos de cambios de secuencias de energización en CS -Doble Terna}

En la Fig. 8, se presentó un ejemplo de valores de campo magnético en cables subterráneos doble terna de 132 kV. Los resultados se presentaron en las Fig. 9 y Fig. 10, se decidió estudiar alternativas de mitigación de campo magnético considerando diferentes secuencias de energización de fases, manteniendo la secuencia de una de las ternas y variando la secuencia de la otra, estas alternativas se presentan en la Tabla V. Para el análisis se considerará al ejemplo de la Fig. 8 como Caso 1.

Tabla V - Alternativas de secuencia de energización para CSAT 2x132 kV, considerando zona de ductos y fosa de empalmes.

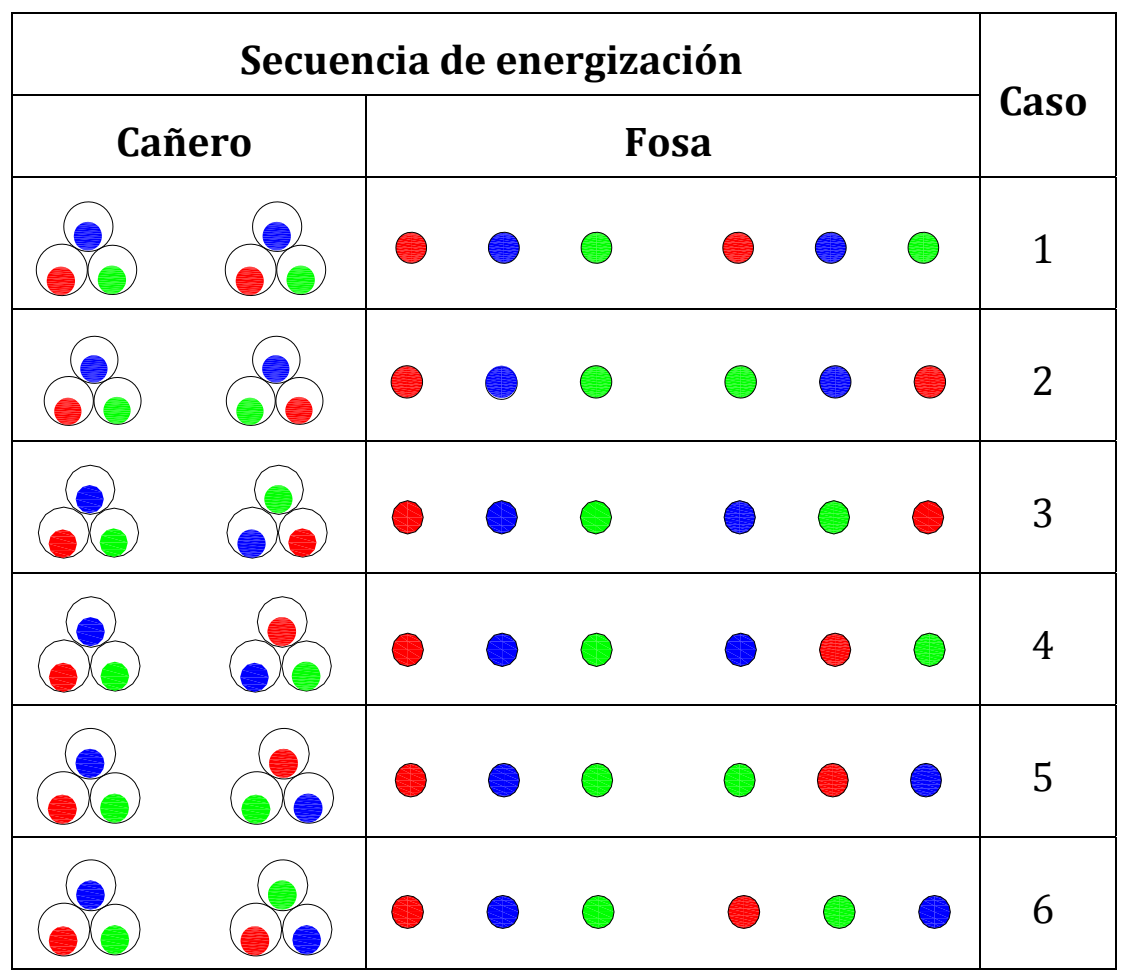




\subsubsection{Resultados Caso 2}

Los resultados obtenidos para el Caso 2 se presentan en las Fig. 29 y Fig. 30. Los mayores valores obtenidos son de 8,5 $\mu \mathrm{T}$ y $14 \mu \mathrm{T}$, en la zona de ductos y fosa respectivamente.

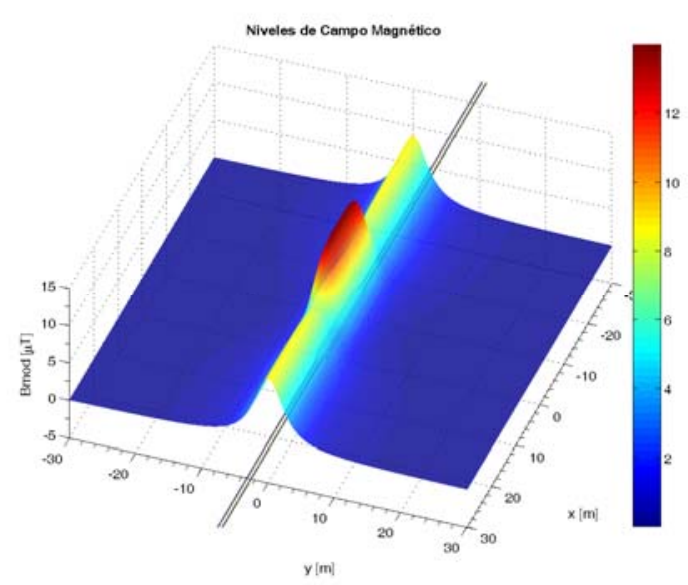

Fig. 29 - Mapa de niveles de B, Caso 2

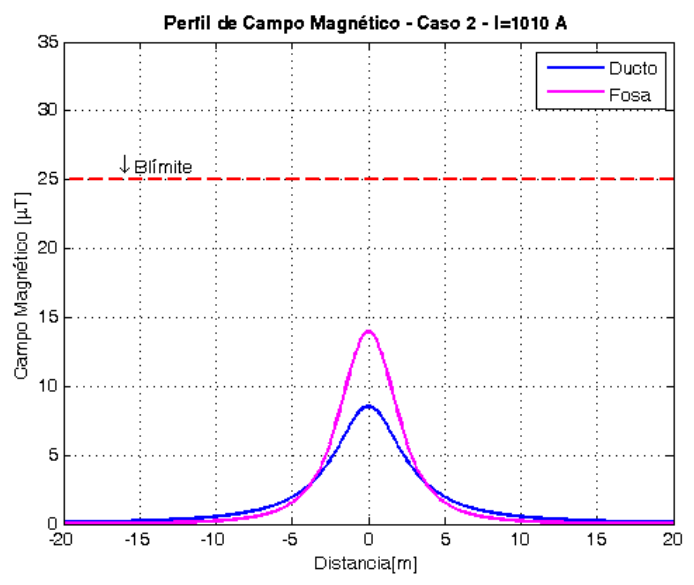

Fig. 30 - Perfiles Transversales, Caso 2

\subsubsection{Resultados Caso 3}

Los resultados obtenidos para el Caso 3 se presentan en las Fig. 31 y Fig. 32. Los mayores valores obtenidos son de $8 \mu \mathrm{T}$ y $20 \mu \mathrm{T}$, en la zona de ductos y fosa respectivamente.

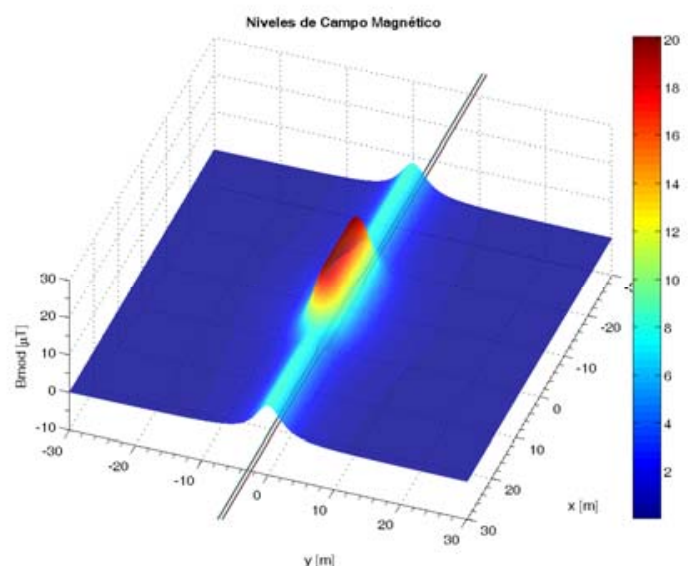

Fig. 31 - Mapa de niveles de B, Caso 23

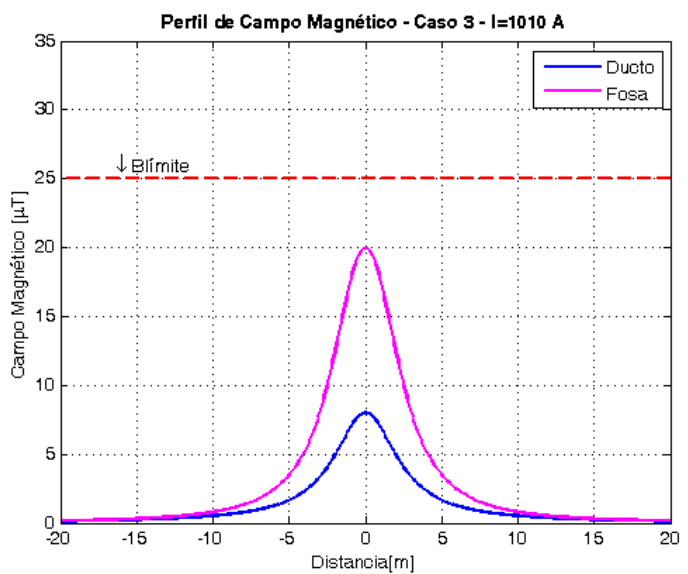

Fig. 32 - Perfiles Transversales, Caso 3

\subsubsection{Resultados Caso 4}

Los resultados obtenidos para el Caso 4 se presentan en las Fig. 33 y Fig. 34. Los mayores valores obtenidos son de $8 \mu \mathrm{T}$ y $25,3 \mu \mathrm{T}$, en la zona de ductos y fosa respectivamente. 


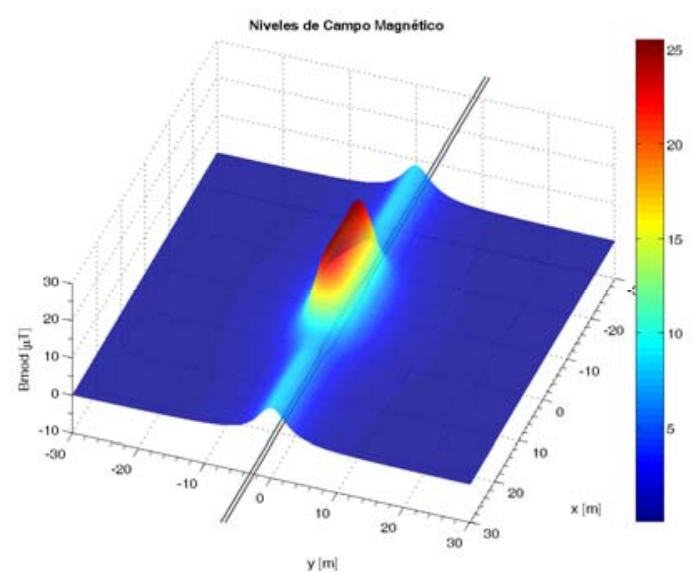

Fig. 33 - Mapa de niveles de B, Caso 4

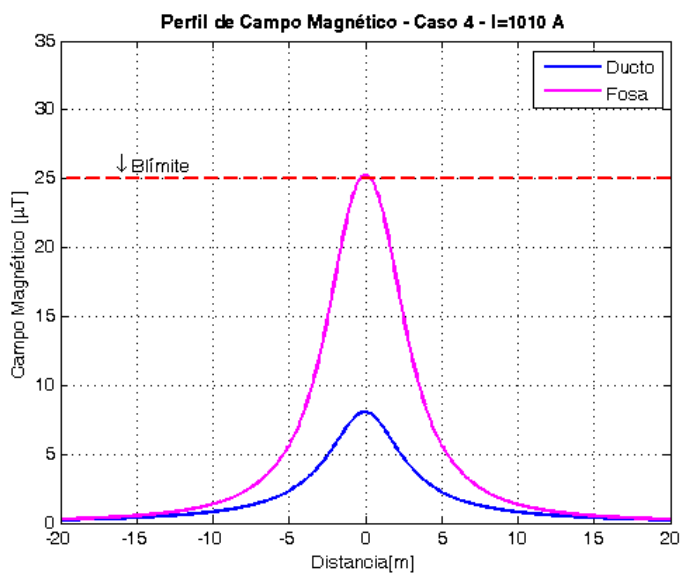

Fig. 34 - Perfiles Transversales, Caso 4

\subsubsection{Resultados Caso 5}

Los resultados obtenidos para el Caso 5 se presentan en las Fig. 35 y Fig. 36. Los mayores valores obtenidos son de $3,3 \mu \mathrm{T}$ y $16,9 \mu \mathrm{T}$, en la zona de ductos y fosa respectivamente.

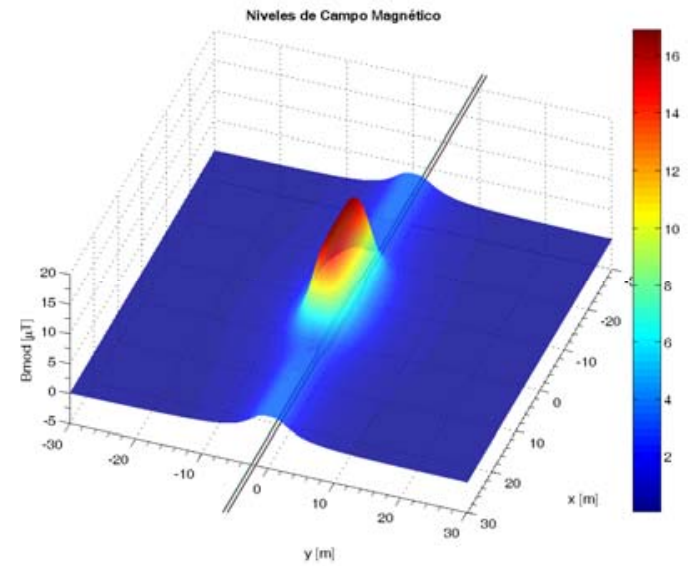

Fig. 35 - Mapa de niveles de B, Caso 5

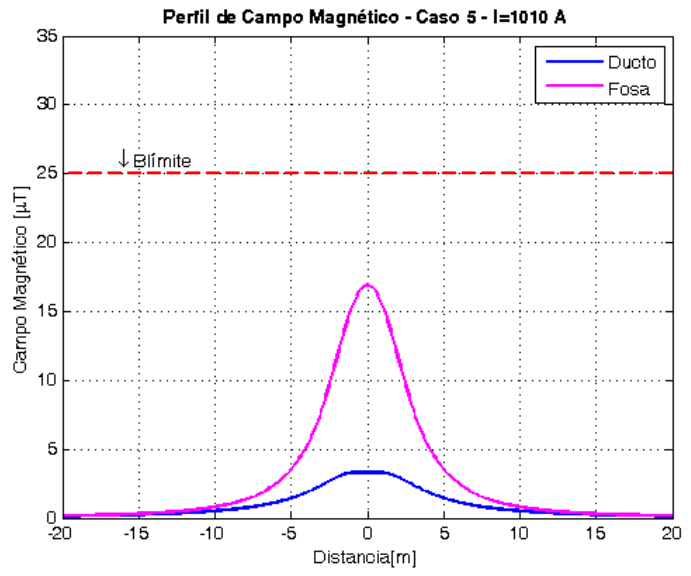

Fig. 36 - Perfiles Transversales, Caso 5

\subsubsection{Resultados Caso 6}

Los resultados obtenidos para el Caso 6 se presentan en las Fig. 37 y Fig. 38. Los mayores valores obtenidos son de $8 \mu \mathrm{T}$ y $25,3 \mu \mathrm{T}$, en la zona de ductos y fosa respectivamente.

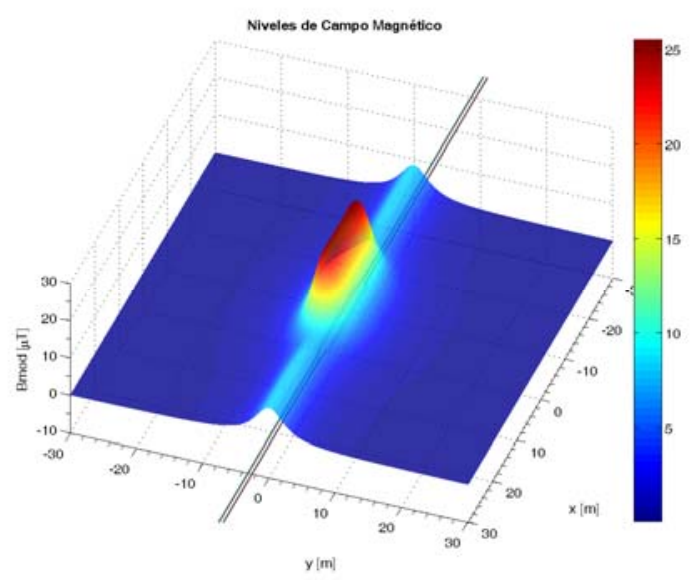

Fig. 37 - Mapa de niveles de B, Caso 6

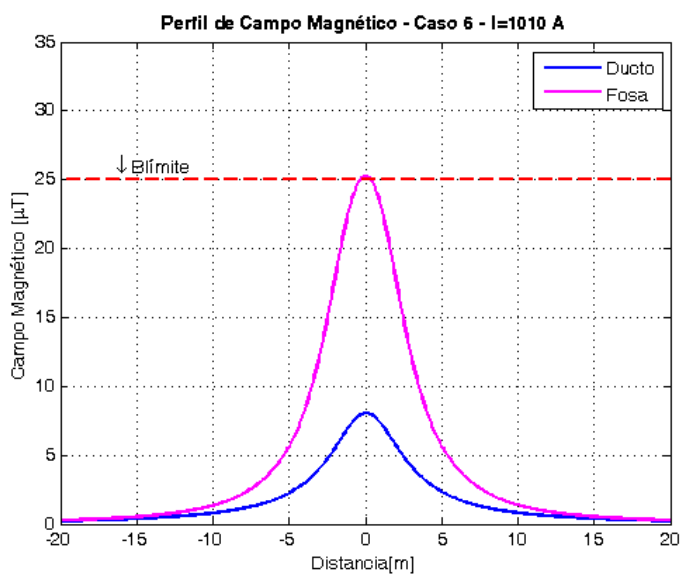

Fig. 38 - Perfiles Transversales, Caso 6 


\subsubsection{Análisis de los resultados}

A continuación se realiza un análisis de los resultados obtenidos, para lo cual se considera el FA, por lo que se trazan los perfiles considerando al Caso 1 como referencia. Se centrará el análisis en la zona de mayor valor de campo magnético $(\mathrm{x}=0)$.

Si se considera la zona de empalmes, Fig. 39, los mejores resultados se obtienen con el Caso 2 , con un FA igual a 2, con lo que se logra una reducción de los valores de campo magnético del $50 \%$, de 28 a $14 \mu \mathrm{T}$.

Los Casos 4 y 6, permiten reducir los valores máximos de campo magnético, no obstante el valor máximo obtenido es mayor que $25 \mu \mathrm{T}$, con lo que estas alternativas no serían una solución al problema planteado, en lo que se refiere a la reglamentación en Argentina.

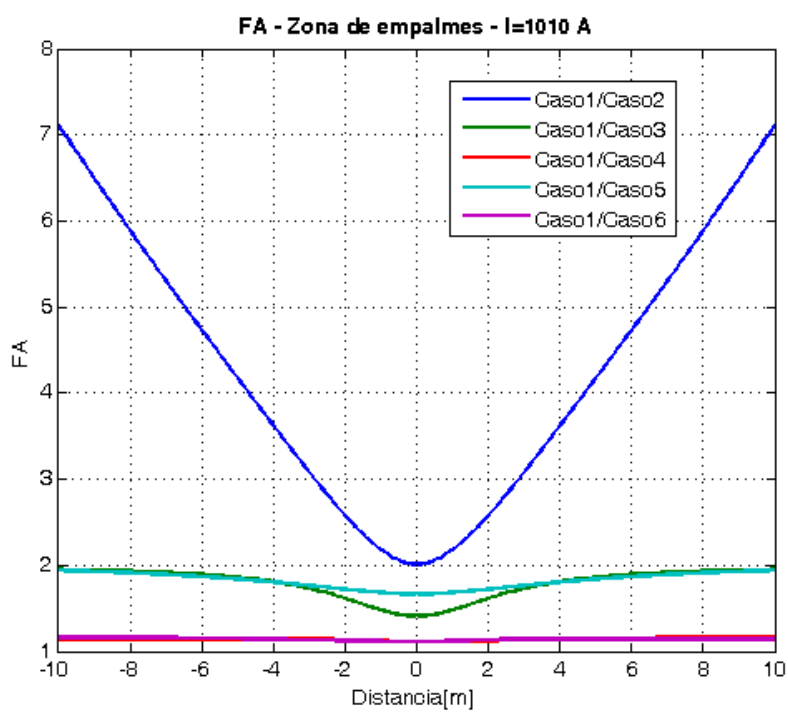

Fig. 39 - FA para zona de empalmes

En la zona de ductos no se superan los $25 \mu \mathrm{T}$ para el Caso 1, no obstante si se analizan los resultados obtenidos, Fig. 40, se observa que la configuración correspondiente al Caso 3 resulta ser la más eficiente, donde el FA máximo es de 3,4.

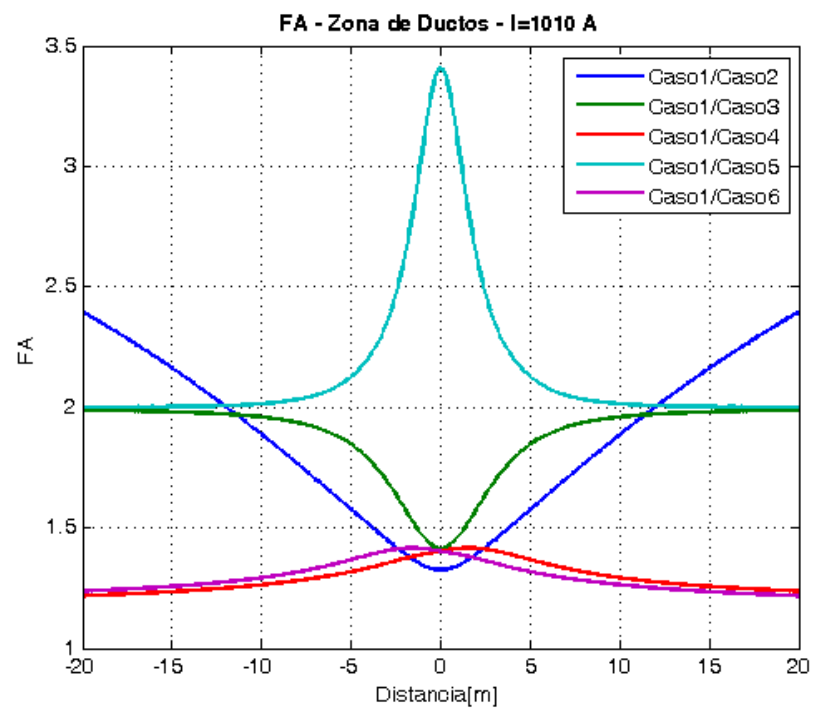

Fig. 40 - FA para zona de ductos 
En la Tabla VI, se presenta un resumen de los resultados obtenidos, se indican los valores máximos de campo magnético y el FA, para zona de ductos y fosa de empalmes, comparados con la configuración del Caso 1.

Tabla VI - Resumen de valores máximos de campo magnético y FA obtenidos, CSAT $2 \times 132 \mathrm{kV}$.

\begin{tabular}{|c|c|c|c|c|}
\hline \multirow{2}{*}{ Caso } & \multicolumn{2}{|c|}{ B máx $[\boldsymbol{\mu T}]$} & \multicolumn{2}{c|}{ FA } \\
\cline { 2 - 5 } & Ducto & Fosa & Ducto & Fosa \\
\hline 1 & 11,3 & 28 & - & - \\
\hline 2 & 8,5 & 14 & 1,3 & 2 \\
\hline 3 & 8 & 20 & 1,4 & 1,4 \\
\hline 4 & 8 & 25,3 & 1,4 & 1,1 \\
\hline 5 & 3,3 & 16,9 & 3,4 & 1,7 \\
\hline 6 & 8 & 25,3 & 1,4 & 1,1 \\
\hline 7 & 11,3 & 10,6 & 1 & 2,65 \\
\hline
\end{tabular}

\subsection{Mitigación utilizando materiales metálicos}

Una estrategia para reducir el campo magnético en una región específica consiste en aprovechar las propiedades de los materiales metálicos (conductividad y permeabilidad) para alterar la distribución espacial del campo magnético producido por una fuente dada [ $11][12][29]$.

Este método requiere la instalación de pantallas metálica entre la fuente de campo y la zona de interés. Pero existen varias posibilidades, dependiendo de las características de la instalación.

Las pantallas metálicas suelen estar compuestas de láminas delgadas, que pueden disponerse como una superficie abierta (blindaje abierto) o para formar una superficie que encierra un volumen de espacio (blindaje cerrado).

Las pantallas cerradas pueden estar dispuestas en dos geometrías alternativas, como se muestra en la Fig. 41. En (a) el campo magnético se impone externamente, por conductores distantes de la zona de interés y un material de blindaje en forma de un rectángulo encierra la región donde se desea la reducción de campo. En la Fig. (b), el material de blindaje encierra los conductores, con una reducción del campo resultante en toda la región fuera de la pantalla. 


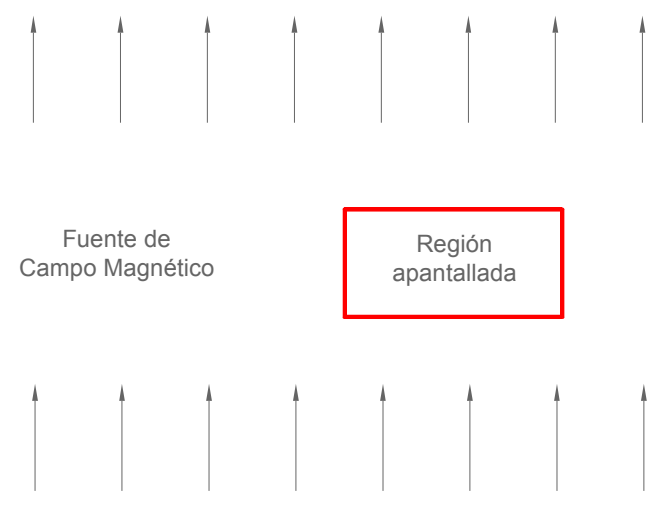

a) Cerramiento del área de interes

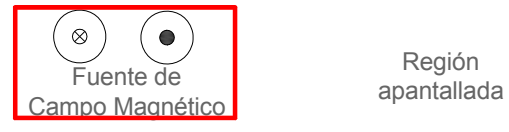

b) Cerramiento de conductores

Fig. 41 - Alternativas de apantallamiento de Campo Magnético, utilizando láminas conductoras.

Dos mecanismos físicos independientes pueden contribuir al blindaje magnético de FEB, considerando los materiales. Los fenómenos físicos involucrados en los efectos de apantallamiento son las corrientes de Foucault en los materiales conductores y, principalmente, el comportamiento ferromagnético en materiales de alta permeabilidad.

Cuando se disponen pantallas metálicas en las proximidades de los conductores de fase de líneas o cables, estas pueden provocar un efecto de mitigación del campo magnético generado por los conductores de fase. Dependiendo de las características de las placas se pueden obtener diferentes resultados [11][12].

Una placa de material de alta permeabilidad puede derivar el flujo, modificando la distribución de las líneas de campo magnético, sin que sea significativo el efecto de corrientes inducidas.

Una placa de material conductor (por ejemplo cobre o aluminio), puede proporcionar blindaje a través de corrientes inducidas.

En general para campos originados por corrientes alternas, el apantallamiento se lleva a cabo mediante configuraciones que utilizan materiales que tienen ambas propiedades, permeabilidad $\mu$ mayor que $\mu_{\mathrm{o}} \mathrm{y}$ conductividad eléctrica lo suficientemente alta como para que se pongan de manifiesto los efectos de corrientes inducidas.

En este punto se describen los resultados encontrados experimentalmente y los obtenidos mediante simulaciones numéricas. Se analiza cómo se altera la distribución del nivel de campo magnético, producido por circuitos recorridos por corrientes de $50 \mathrm{~Hz}$, ante la presencia de blindajes.

Para el análisis se implementó en laboratorio un sistema de conductores con geometría y corriente de magnitud controlada. Se realizaron determinaciones del nivel de campo en el entorno del sistema sin la presencia de las placas de blindaje y luego con las mismas. Los resultados obtenidos se utilizan a fin de validar el modelo numérico de simulación mediante el método de elementos finitos en 3D.

Una vez validado el modelo se analizó el caso de un puesto de transición típico, correspondiente a doble terna de línea aérea - cable subterráneo en $132 \mathrm{kV}$. Los cálculos se realizan contemplando distintas alternativas. El blindaje se implementa con placas metálicas empleadas en la protección mecánica de puestos de transición. 


\subsubsection{Experiencias en laboratorio}

El primer paso consistió en validar la simulación numérica. Para esto se implementó una geometría que resultara similar a los casos reales y que a su vez pudiera ejecutarse prácticamente con el máximo grado de control de sus parámetros tanto geométricos como eléctricos.

Para las determinaciones experimentales se preparó una configuración que se ajustó a la geometría y excitación eléctrica igual a la empleada en las simulaciones numéricas como se muestra en la Fig. 42.

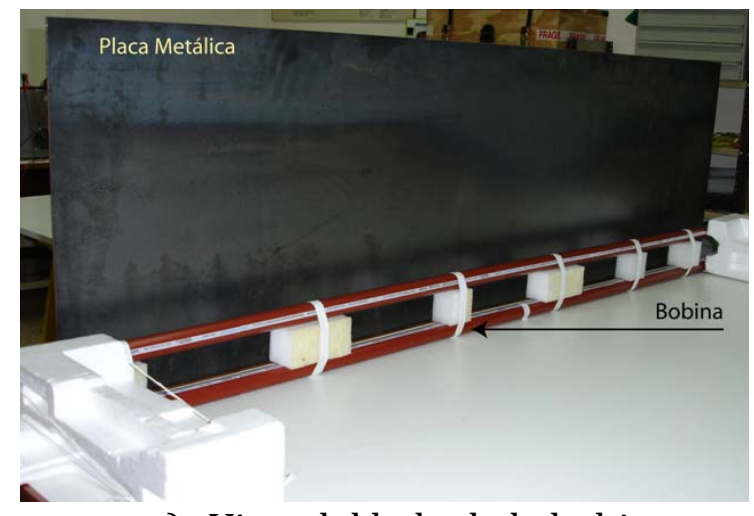

a) Vista del lado de la bobina

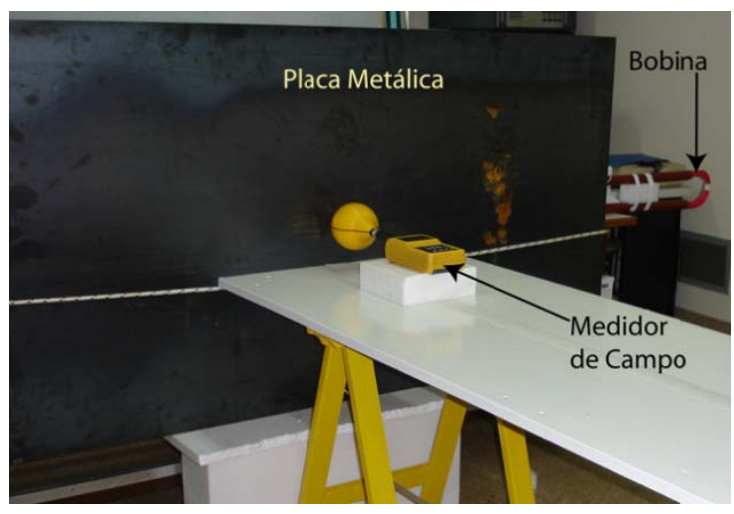

b) Vista del lado de medición

Fig. 42 - Modelo experimental de espira y placa de blindaje.

Para que los resultados fueran comparativos, en todos los casos verificados, se mantuvo por la espira generadora de campo una corriente de $200 \mathrm{~A}$ y $50 \mathrm{~Hz}$.

Se utilizaron placas de $2 \mathrm{~m}$ de largo y $1 \mathrm{~m}$ de alto con diferentes espesores (e), características de conductividad eléctrica $(\sigma)$, permeabilidad relativa $\left(\mu_{\mathrm{r}}\right)$ y profundidad de penetración $(\rho)$ en $50 \mathrm{~Hz}$. Las placas se dispusieron paralelas a la espira a 0,1 m de la misma.

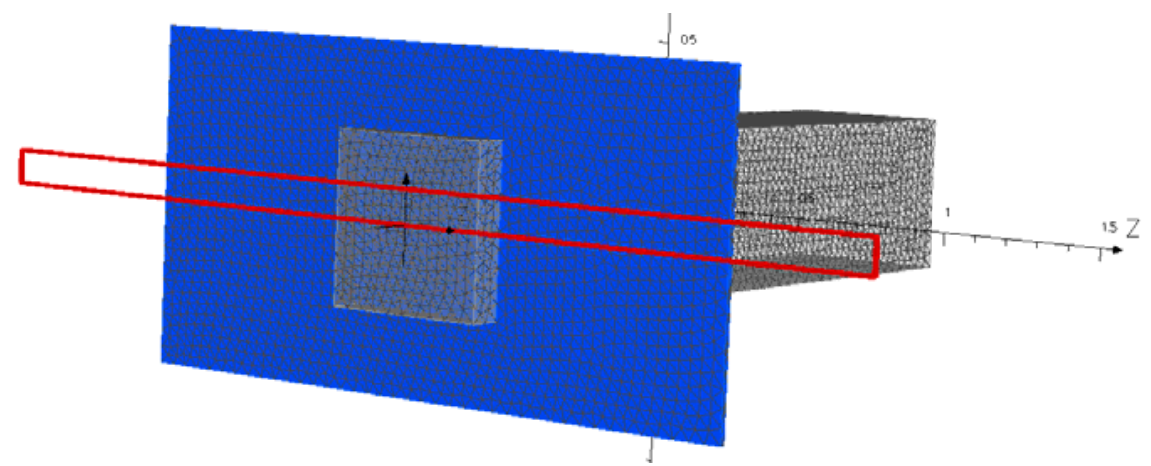

Fig. 43 - Modelo de simulación de espira (Rojo) y placa de blindaje (Azul).

A los efectos de este trabajo, sólo se presentan algunos de los casos analizados que se vuelcan en la Tabla VII.

Tabla VII - Modelo experimental de espira y placa de blindaje, resumen de los valores experimentales.

\begin{tabular}{|l|c|c|c|c|}
\hline \multicolumn{1}{|c|}{ Material } & $\mathbf{e}[\mathbf{m m}]$ & $\sigma[\mathbf{S} / \mathbf{m}]$ & $\mu_{\mathbf{r}}$ & $\delta$ @ $\mathbf{5 0 ~ H z}$ \\
\hline Aluminio(a) & 1,5 & $35 \cdot 10^{6}$ & 1 & $12 \mathrm{~mm}$ \\
\hline Hierro(a) & $1 \mathrm{a} 4,5$ & $8,4 \cdot 10^{6}$ & 260 & $1,5 \mathrm{~mm}$ \\
\hline Hierro(b) & 2,5 & $8,4 \cdot 10^{6}$ & 240 & $1,5 \mathrm{~mm}$ \\
\hline $\begin{array}{r}\text { (a) Datos correspondientes a materiales empleados en laboratorio. } \\
\text { (b) }\end{array}$ & Datos correspondientes a materiales empleados instalaciones reales. \\
\hline
\end{tabular}


En la Fig. 44 se presentan los valores de campo magnético obtenidos a través de cálculos, considerando placas de diferente espesor de un mismo material (ferromagnético); en Fig. 45 se presentan los valores de FA correspondientes.

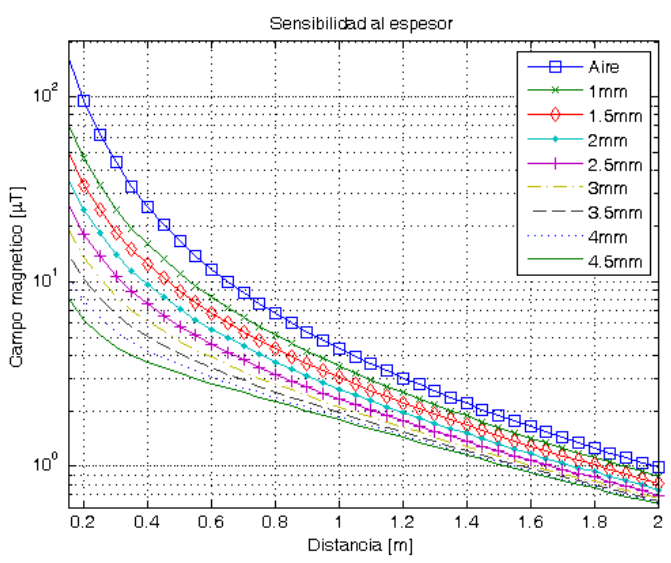

Fig. 44 - Campo magnético simulado considerando placas de distintos espesores.

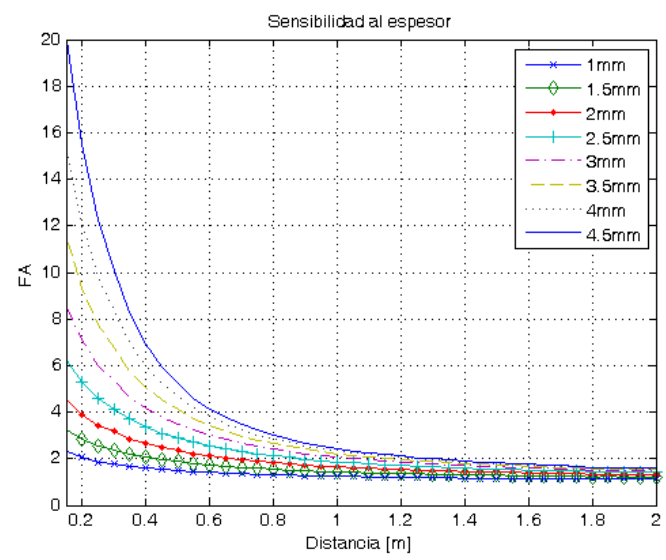

Fig. 45 - FA considerando valores simulados, considerando placas de distintos espesores.

En la Fig. 46 se presentan los resultados obtenidos en mediciones y simulaciones, sin placa y para placas de diferentes materiales. Los resultados obtenidos están dentro del $20 \%$ de lo esperado. En la Fig. 47 se presentan los valores obtenidos para FA considerando únicamente los valores obtenidos en las simulaciones.

Las experiencias realizadas en laboratorio permitieron validar los modelos de cálculo, comparando los resultados obtenidos con mediciones. En primer lugar se consideró la presencia de la espira como fuente de campo magnético sin placas metálicas. Luego se incorporaron placas de diferentes materiales y espesores.

La evaluación de sensibilidad realizada para distintos espesores de chapa (Fig. 44 y Fig. 45), permite asegurar que la mejora en la mitigación del campo magnético se obtiene hasta espesores de chapa del orden del doble de la profundidad de penetración por efecto pelicular. En el caso de los materiales utilizados en protecciones mecánicas el valor correspondiente de $\delta$ es de 1,5 mm, a $50 \mathrm{~Hz}$. Espesores mayores no aportan beneficios superiores.

En la Fig. 46 se presenta una comparación entre los valores de campo magnético obtenidos en mediciones y simulaciones, en la Fig. 47 se presentaron los valores de FA correspondientes considerando placas de Hierro y Aluminio.

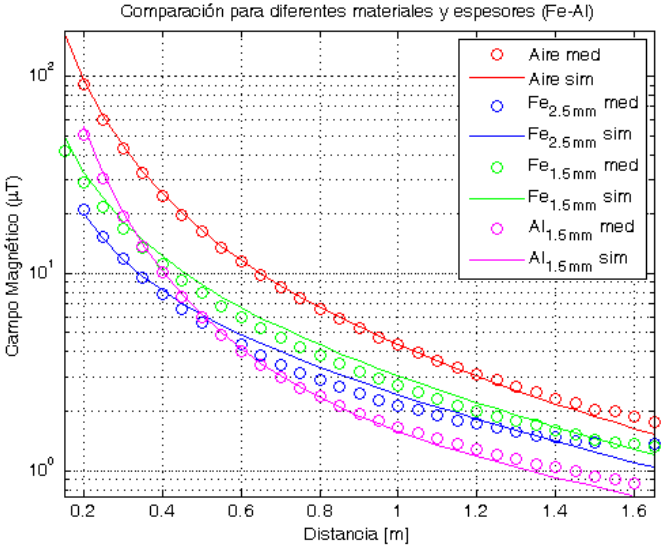

Fig. 46 - Campo magnético medido y simulado (Aire, Fe, Al), con diferentes placas.

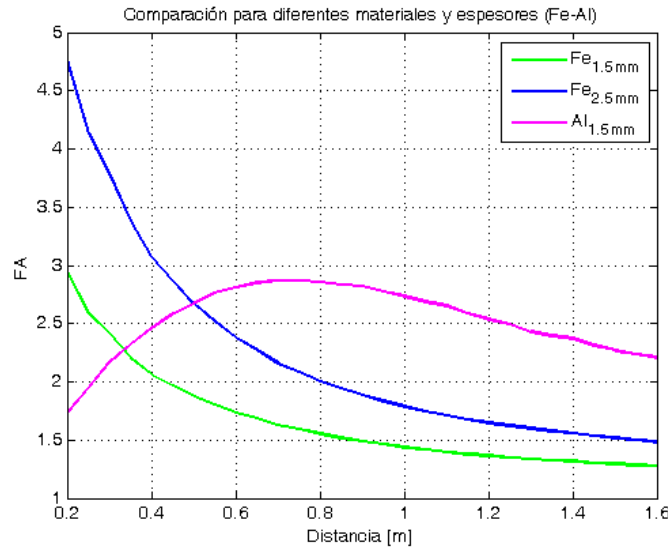

Fig. 47 - FA considerando valores simulados (Aire, $\mathrm{Fe}, \mathrm{Al}$ ), considerando diferentes placas. 
Uno de los materiales considerados fue aluminio, cuya principal características de influencia es la conductividad, $\sigma=35 \cdot 10^{6} \mathrm{~S} / \mathrm{m}$ y $\mu_{\mathrm{r}}=1$, las corrientes inducidas son las que proporcionan el mecanismo de mitigación del campo magnético.

Las demás placas consideradas fueron de hierro una de ellas corresponde al material y espesor típicos que se utilizan para brindar protección mecánica en puestos de transición entre líneas aéreas y cables subterráneos, las características son $\sigma=8,4 \cdot 10^{6} \mathrm{~S} / \mathrm{m}$ y $\mu_{\mathrm{r}}=240$. Si se comparan las dos placas de hierro, se observa que la de mayor espesor $(2,5 \mathrm{~mm})$ es la que presenta un mejor desempeño, esto se debe a que este es levemente inferior al doble de la profundidad de penetración.

El parámetro más importante de este material es la conductividad. Una mayor conductividad, implica un mejor blindaje. El material considerado no posee una permeabilidad relativa demasiado elevada, por lo que predomina el efecto de inducción por sobre el efecto magnético. En la Fig. 48 se presenta una imagen con la distribución de corrientes inducidas en la placa cuyas características son $\sigma=8,410^{6} \mathrm{~S} / \mathrm{m}, \mu_{\mathrm{r}}=240$ y espesor $2,5 \mathrm{~mm}$. En diferentes colores se aprecia la densidad de corriente y además se presentan los vectores correspondientes. En color rojo se representa la espira en la zona central.

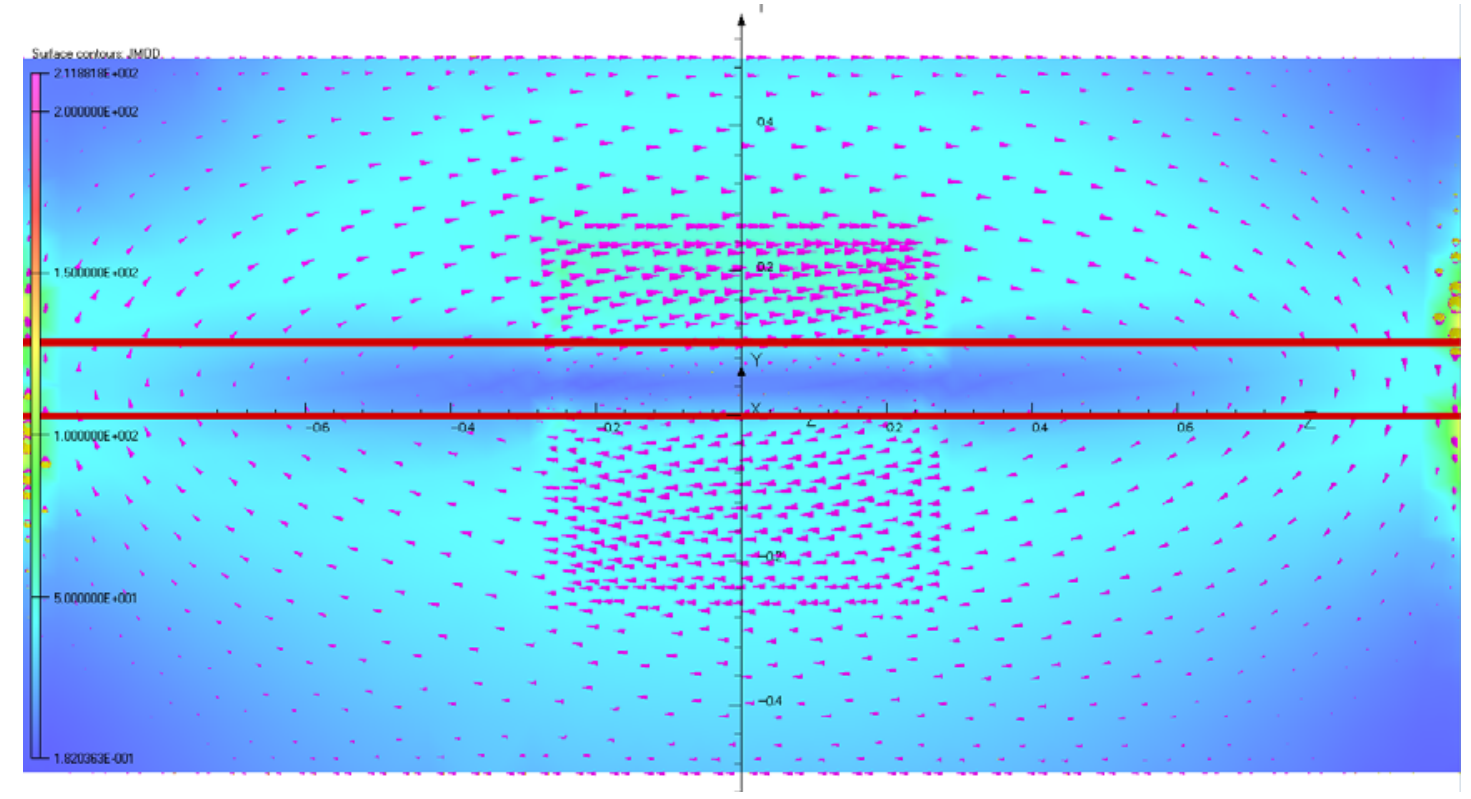

Fig. 48 - Densidad de corriente en placa cuyas características son $\sigma=8,410^{6} \mathrm{~S} / \mathrm{m}, \mu_{\mathrm{r}}=240$ y espesor igual a $2,5 \mathrm{~mm}$.

\subsubsection{Aplicación práctica}

En este punto se presenta una aplicación práctica de la utilización de placas metálicas para mitigación de campo magnético. Se considera un puesto de transición entre LAAT y CSAT, con dos ternas.

Una vez realizada la evaluación del comportamiento del material típico que se utiliza en protecciones mecánicas de puestos de transición $\left(\sigma=8,4 \cdot 10^{6} \mathrm{~S} / \mathrm{m}, \mu_{\mathrm{r}}=240\right.$ y espesor $\left.2,5 \mathrm{~mm}\right)$, se decidió analizar la implementación en una instalación. Definidas las características del material, se evaluó el comportamiento para diferentes dimensiones.

En las Fig. 49 y Fig. 50 se aprecian las características principales del puesto de transición. Desde la parte superior las dos ternas (Rojo), en la parte central la estructura metálica cilíndrica (Verde) y a ambos lados las protecciones metálicas (Azul). La protección está compuesta por dos placas de hierro que rodean los cables de bajada. Se estudia la influencia 
de distintas configuraciones geométricas, analizando distintos valores de relación de ancho y separación, indicados en la Fig. 49 como DX y DY. La altura de la protección mecánica es de 2 metros y el espesor de $2.5 \mathrm{~mm}$.

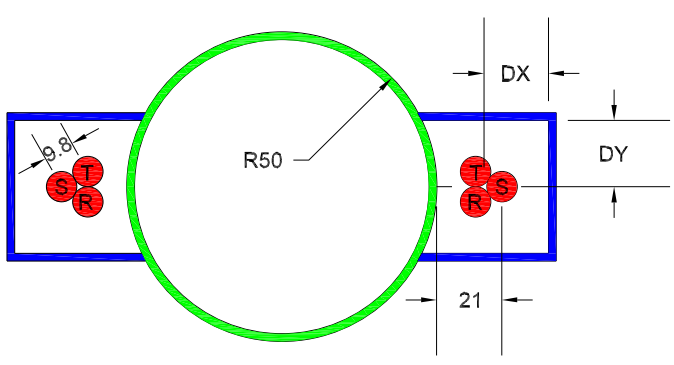

Fig. 49 - Vista superior de la geometría del puesto de transición, conductores (Rojo), estructura metálica (Verde) y protección mecánica (Azul).

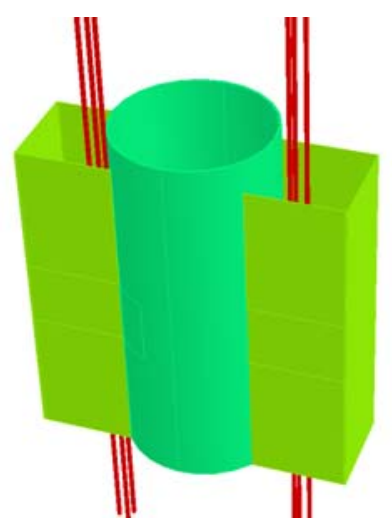

Fig. 50 - Modelo que incluye los conductores de bajada, la protección mecánica y la torre.

En el Anexo VIII se presenta un detalle de las condiciones de borde impuestas para resolver el problema.

En la Fig. 50 se presenta una parte del modelo, en el que se observa una parte de la estructura, los conductores y las placas.

Se analizaron diversos casos, en primer lugar no se consideró la existencia de la torre ni de la placa. Luego se observó únicamente la existencia de la torre, y finalmente luego se consideraron diferentes dimensiones para la protección mecánica. En la Tabla VIII se resumen los casos considerados.

Además la corriente considerada fue de 200 A y se contemplaron desbalances del $5 \%$ en la componente de secuencia inversa.

Tabla VIII - Resumen casos de corriente analizados.

\begin{tabular}{|c|c|c|c|}
\hline \multirow{2}{*}{ Caso } & \multirow{2}{*}{$\begin{array}{c}\text { Dimensiones } \\
{[\mathbf{c m}]}\end{array}$} & \multicolumn{2}{|c|}{ Corrientes/Secuencia } \\
\cline { 3 - 4 } & Directa & Inversa \\
\hline 0 & Sin torre ni placa & $200 \mathrm{~A}$ & $0 \%$ \\
\hline 1 & Sin placas & $200 \mathrm{~A}$ & $0 \%$ \\
\hline 2 & Sin placas & $200 \mathrm{~A}$ & $5 \%$ \\
\hline 3 & $\mathrm{DX}=20 / \mathrm{DY}=20$ & $200 \mathrm{~A}$ & $0 \%$ \\
\hline 4 & $\mathrm{DX}=20 / \mathrm{DY}=20$ & $200 \mathrm{~A}$ & $5 \%$ \\
\hline 5 & $\mathrm{DX}=30 / \mathrm{DY}=30$ & $200 \mathrm{~A}$ & $0 \%$ \\
\hline 6 & $\mathrm{DX}=30 / \mathrm{DY}=30$ & $200 \mathrm{~A}$ & $5 \%$ \\
\hline
\end{tabular}

Los resultados son presentados en forma de curvas de nivel y corresponden a una vista en planta de los valores de campo magnético resultante a un metro sobre el nivel del suelo. Debe destacarse que no existe simetría en ningún caso debido a la influencia de la línea aérea ubicada en la parte superior de la torre y el cable subterráneo en la parte inferior de la misma, que fueron representados según el correspondiente tendido. 
El Caso 0, Fig. 51, refleja los valores de campo presentes en los alrededores de la bajada sin considerar el efecto de reducción de las corrientes que se inducen en la torre y en las protecciones mecánicas.

El Caso 1, Fig. 52, muestra el campo magnético sin tener en cuenta los efectos de la protección mecánica pero considerando la torre. Como se ve, la torre no produce una reducción importante de los valores de campo en su exterior.

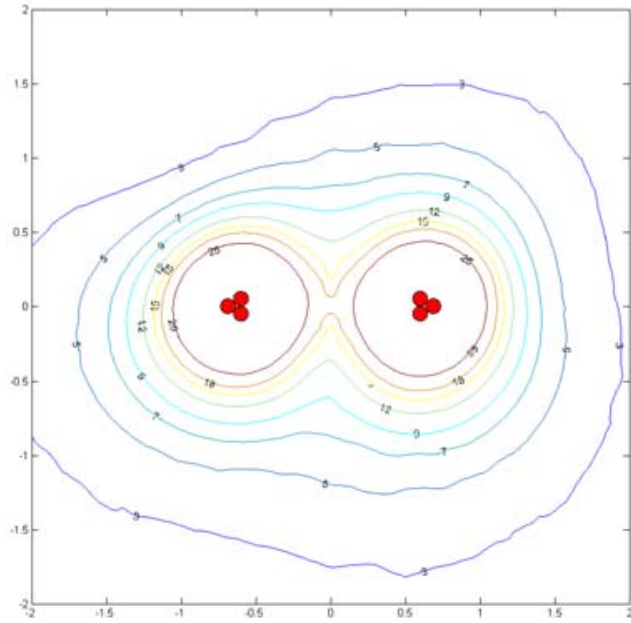

Fig. 51 - Resultados obtenidos para el Caso 0, curvas de nivel $B_{R}$.

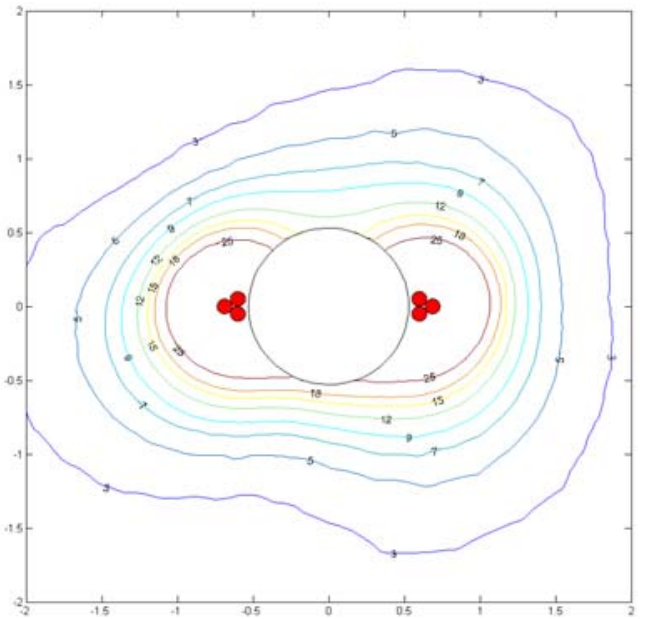

Fig. 52 - Resultados obtenidos para el Caso 1 , curvas nivel $\mathrm{B}_{\mathrm{R}}$.

La Fig. 53 corresponde al Caso 2 que es idéntico al Caso 1 pero con un desbalance. Se puede ver el campo magnético sufre un incremento con respecto al caso balanceado (Fig. 52).

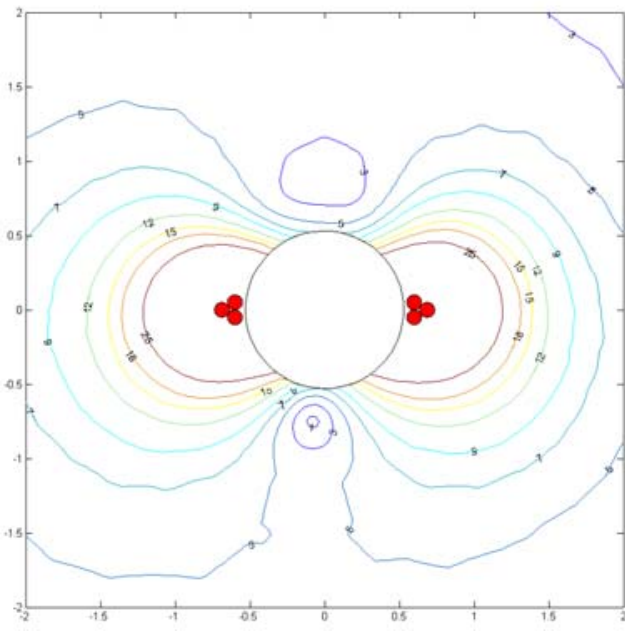

Fig. 53 - Resultados obtenidos para el Caso 2, curvas de nivel $\mathrm{B}_{\mathrm{R}}$.

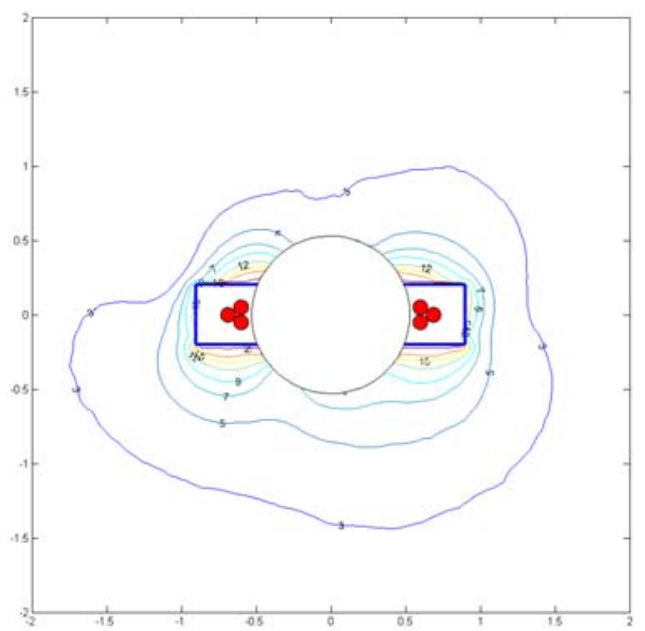

Fig. 54 - Resultados obtenidos para el Caso 3, curvas de nivel $\mathrm{B}_{\mathrm{R}}$.

El Caso 3 (sin desbalance), Fig. 54, muestra que la presencia de la protección mecánica reduce de manera notable los campos magnéticos. La línea de isocampo magnético de $25 \mu \mathrm{T}$ queda prácticamente confinada dentro de la protección mecánica.

La Fig. 55 corresponde al Caso 4 considerando desbalance. Aquí podemos ver que la línea de $25 \mu \mathrm{T}$ se escapa ligeramente de la protección. 


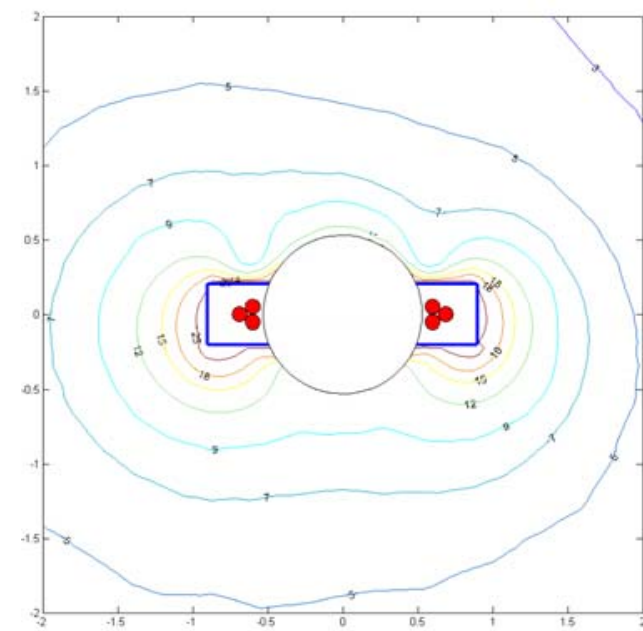

Fig. 55 - Resultados obtenidos para el Caso 4, curvas de nivel $B_{R}$.

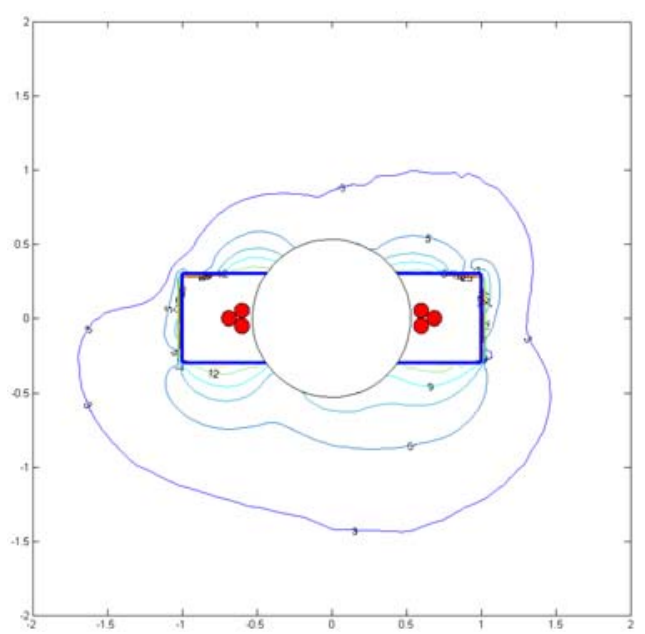

Fig. 56 - Resultados obtenidos para el Caso 5, curvas de nivel $B_{R}$.

El Caso 5 sin desbalance se presenta en la Fig. 56. En ella se puede ver que la protección mecánica reduce aún más los campos magnéticos, quedando la línea de isocampo magnético de $25 \mu$ T totalmente dentro de la protección mecánica.

El mismo resultado se obtiene en el Caso 6 considerando desbalance, Fig. 57. Se aprecia que si bien los campos son mayores que en el Caso 5, la línea de $25 \mu \mathrm{T}$ no excede los límites de protección mecánica.

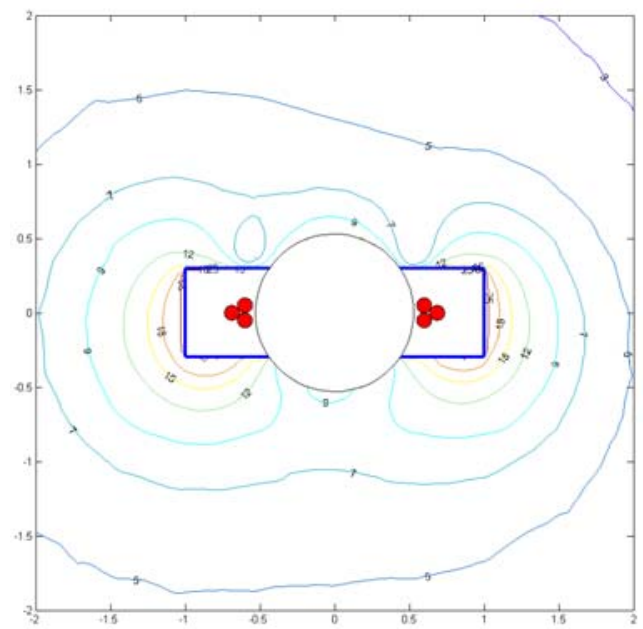

Fig. 57 - Resultados obtenidos para el Caso 6, curvas de nivel $B_{R}$.

Para el ejemplo considerado en este punto se evaluaron los resultados obtenidos en los diferentes casos.

Se consideró en primer lugar la distribución de campo magnético generado únicamente por los conductores de fase. Luego se consideró la presencia de la torre en el puesto de transición. En ambos casos se observa que el valor de $25 \mu \mathrm{T}$ se supera a distancias relativamente pequeñas de la instalación, menos de $0,5 \mathrm{~m}$. Si no se disponen protecciones mecánicas puede considerarse que el perímetro de la instalación sería accesible al público en general y no se cumpliría con el valor de campo exigido por la reglamentación.

El objetivo planteado fue dimensionar las protecciones mecánicas, una vez seleccionado el material, de forma tal que los valores de campo magnético en el exterior de la misma, se limiten a valores inferiores a $25 \mu \mathrm{T}$, exigidos por la reglamentación vigente. Se consideraron diferentes condiciones de carga y diferentes dimensiones para las protecciones mecánicas. 
El objetivo se logró con protecciones mecánicas cuyas dimensiones fuero DX=30 cm y $\mathrm{DY}=30 \mathrm{~cm}$.

\subsubsection{Comentarios}

En este punto se consideró la posibilidad de mitigar el campo de inducción magnética por medio de la utilización de blindajes pasivos, implementados con placas de materiales de uso habitual en las instalaciones eléctricas. Esto trae aparejado una solución técnico-económica viable y de sencilla implementación.

Se utilizaron herramientas de simulación, validadas por mediciones, para realizar la evaluación de distintas alternativas de mitigación y seleccionar la que presente mejor performance.

Los cables en los puestos de transición deben contar con una protección mecánica. Dicha protección puede ser utilizada en forma eficiente para mitigar el campo magnético generado por los conductores de manera de cumplir con la reglamentación vigente.

Para un determinado material, a medida que se incrementan las dimensiones de la protección se logra un mejor efecto de mitigación. Es posible conseguir que los valores límites de $25 \mu \mathrm{T}$, reglamentados en Argentina, queden confinados al interior de las protecciones mecánicas.

En las placas consideradas en el ejemplo ( $\mathrm{e}=2,5 \mathrm{~mm}, \sigma=8,4 \times 10^{6} \mathrm{~S} / \mathrm{m}$ y $\left.\mu_{\mathrm{r}}=240\right)$, las corrientes inducidas producen un campo magnético que cancela parcialmente al campo original. De esta forma se logra un efecto de mitigación del campo magnético en el exterior de las protecciones mecánicas. La permeabilidad del material considerado es relativamente baja por lo que los efectos de las corrientes inducidas son los que prevalecen por sobre los magnéticos.

\subsection{Lazos Pasivos}

La compensación por medio de lazos pasivos consiste en disponer un conductor en forma de espira o lazo, en una posición tal que se induzca una tensión, como consecuencia del concatenamiento del flujo de campo magnético, " $B_{R}$ ", generado por los conductores de fase de una línea de transmisión. Como resultado de esta tensión y considerando la impedancia del lazo se produce una circulación de corriente en dicho lazo.

Las corrientes inducidas en los conductores de compensación dan origen a un campo magnético que anula parcialmente el campo original.

El estudio detallado de esta estrategia de mitigación es el principal objeto de este trabajo, por lo que será abordado en detalle en los capítulos siguientes.

\subsection{Lazos Activos}

Es posible aumentar la eficacia de la mitigación si, por medio de una fuente de alimentación externa, se inyecta un valor de corriente apropiado en una bobina o lazo de compensación. El valor de corriente en magnitud y fase tiene que ser calculado con el fin de obtener la máxima mitigación en el punto o área de interés. El valor de dicha corriente depende directamente del valor de campo magnético, y mientras este varía, la corriente de bucle de compensación debe cambiar en consecuencia. Por lo tanto, es necesario algún tipo de sistema de control, como ser una fuente de corriente externa electrónicamente controlada y sincronizada con el campo original.

En la Fig. 58 se presenta un esquema de compensación mediante lazo activo, se observa una fuente externa de campo magnético y un área de interés. Entre estos se dispone de un lazo de 
compensación. En la zona de interés es necesario un sensor de campo que permite monitorear cual es el valor de campo en el interior de la misma. Dicho valor se ingresa en un sistema de control, que actúa sobre una fuente de alimentación, que a su vez permite regular la corriente por el lazo activo en módulo y fase.

Si la fuente externa de campo magnético presenta un comportamiento conocido, como por ejemplo una línea, en la cual el valor de campo magnético depende de la corriente por la misma, el sensor de campo magnético en la zona de interés puede ser remplazado por algún dispositivo de medición de corriente o campo sobre la línea.

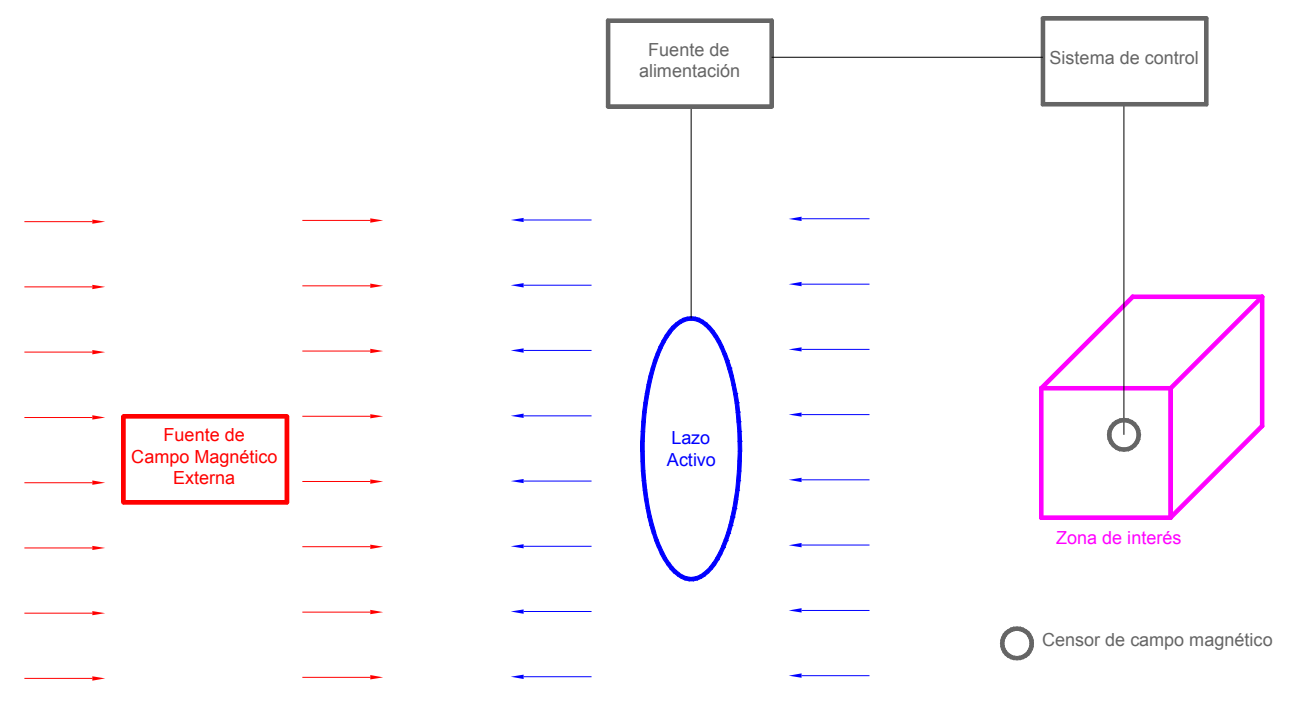

Fig. 58 - Compensación con lazo activo

Es posible señalar algunas diferencias con este método y la compensación con lazos pasivos:

- El sistema de lazos pasivos es un sistema autorregulado ya que siempre que la fuente presente cambios, las corrientes por el o los lazos varía de una manera natural. El sistema de lazo activo necesita un sistema de regulación adicional.

- La ubicación de un lazo activo no es tan crítico como la de uno pasivo.

- Con el sistema de lazos activos no resulta crítico el valor de resistencia del lazo para obtener una buena efectividad en la mitigación.

- Debido a que la corriente inyectada es calculada y controlada, la mitigación es en general mayor que la alcanzada con lazos pasivos.

- La necesidad de un equipo externo hace que esta solución resulte más costosa y menos fiable que el lazo pasivo. 


\section{Lazos Pasivos}

En esta sección se analiza una técnica de mitigación de campo magnético, basada en la disposición de lazos pasivos en los que se inducen corrientes, debido a la circulación de corrientes de los conductores de fase de una línea [11 ] [ 30 ] [ 27 ] [31 ] [ 32 ] [ 33 ] [ 12 ] [ 34 ].

Se pretende conocer cuáles son las configuraciones a que esta técnica es aplicable, hallar la posición más adecuada para la disposición de los lazos y las principales características de los conductores empleados en ellos.

Para abordar esta etapa es necesario considerar en primer lugar las principales características del campo magnético que se desea mitigar y en función de este, diseñar el sistema de lazos que produzca la mitigación óptima del campo magnético.

\subsection{Generalidades}

La compensación por medio de lazos pasivos consiste en disponer un conductor en forma de espira o lazo, en una posición tal que se induzca una tensión, como consecuencia del concatenamiento del flujo de campo magnético, " $\mathrm{B}_{\mathrm{r}}$ ", generado por los conductores de fase de una línea de transmisión. Como resultado de esta tensión y considerando la impedancia del lazo se produce una circulación de corriente en dicho lazo.

Esta corriente crea un campo magnético " $\mathrm{B}_{\mathrm{i}}$ " que se opone al campo original " $\mathrm{B}_{\mathrm{r}}$ ", esto arroja como resultado un campo resultante " $\mathrm{B}_{0}$ ".

Para poder cuantificar este efecto se deben conocer además de las características constructivas de los conductores de fase, las características del o los lazos, considerando los factores que se describen a continuación:

- Corriente por los conductores de fase.

- Distancia desde el conductor de fase al lazo.

- Resistividad del conductor utilizado para el lazo.

- Sección del conductor

- Longitud y ancho del lazo

- Impedancia del lazo

Estas magnitudes permiten calcular el flujo que atraviesa el lazo debido a los conductores de fase, la tensión inducida y así conocer la corriente que circulará en el lazo.

\subsubsection{Hipótesis para el cálculo de la corriente inducida en un lazo}

Para simplificar el cálculo se consideran algunas hipótesis que se enumeran a continuación:

- Se consideran que los conductores de fase son rectos, paralelos e infinitos.

- Los lazos considerados están compuestos por cuatro tramos rectos, paralelos de a pares, dos de ellos paralelos a los conductores de fase y otros dos perpendiculares.

- La longitud de los tramos paralelos son, largo del lazo, son mucho mayores que los tramos perpendiculares, ancho del lazo.

- La tensión inducida en los conductores paralelos a los conductores de fase es mucho mayor que la inducida en los tramos perpendiculares.

- La distancia entre cualquiera de los conductores de fase y los del lazo es al menos un orden de magnitud mayor que el radio del conductor, esto permite despreciar los efectos de proximidad entre diferentes conductores. 
- La corriente de los conductores de fase no se modifica por la inserción de lazos pasivos. Este efecto adquiere mayor relevancia a medida que la longitud del lazo se aproxime a la longitud de la línea.

- La tierra se considera horizontal, homogénea e infinita. Esto permite simplificar el problema a dos dimensiones, despreciando la coordenada en la dirección de la línea.

\subsubsection{Principales características de un lazo pasivo}

Se denomina corriente inducida en el lazo a la corriente que circulará por el mismo como consecuencia de la aparición de una tensión inducida, producto del flujo concatenado, y la impedancia del lazo.

Para determinar esta corriente hay que entender algunas cuestiones que a continuación se detallan.

En primer lugar hay que establecer un sistema de coordenadas en el cual se hallen identificados cada uno de los conductores, en primera instancia se considera un solo conductor de fase y un lazo.

En la Fig. 59 puede apreciarse el campo magnético $\left(\mathrm{B}_{\mathrm{r}}\right)$ debido al conductor " $\mathrm{r}$ ", se representan dos líneas de campo concéntricas con el mismo. Se considera que por dicho conductor circula una corriente " $\mathrm{I}_{\mathrm{r}}$ ", con sentido entrante a la página. El campo magnético debido a la corriente por el conductor "r" esta dado por la Ec. 4.

$$
B_{r}=\frac{\mu_{0} I_{r}}{2 \pi r}
$$

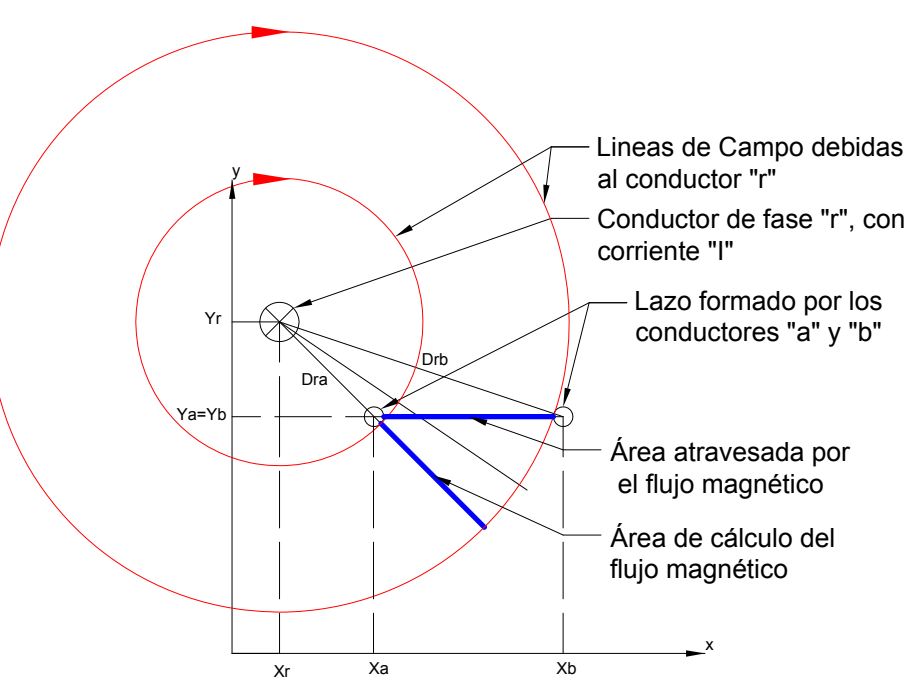

Fig. 59 - Flujo concatenado en un circuito externo, debido a la corriente "I", por un conductor "r".

Lo que se busca es determinar el flujo que atraviesa el bucle "a-b", debido a la corriente Ir, este flujo es igual al flujo que pasa entre las superficies coaxiales trazadas a distancias Dra y Drb, centradas en el conductor " $r$ ", cuyas coordenadas son (Xr,Yr).

\subsubsection{Flujo concatenado " $\Phi$ "}

Para estimar el flujo concatenado con la espira "a-b" hay que calcular la componente perpendicular del mismo con el plano de la espira. En la Fig. 59 "Área atravesada por el flujo magnético" (Área I) [ 35 ]. 
Considerando el concepto de tubos de flujo, se observa que el flujo que atraviesa la superficie de la espira, "Área I", es el mismo que atraviesa el "Área de cálculo de flujo magnético" (Área II). Pero en esta última las líneas de flujo son perpendiculares, entonces es posible determinar de una forma más sencilla el flujo total concatenado.

El módulo del flujo está dado por la Ec. 5.

$$
\Phi_{r a b}=l \int_{D_{r a}}^{D_{r b}} B_{r} d r=l \int_{D_{r a}}^{D_{r a}} \frac{\mu_{0} I_{r}}{2 \pi r} d r=l \frac{\mu_{0} I_{r}}{2 \pi} \ln \frac{D_{r b}}{D_{r a}}=2 \times 10^{-7} I_{r} l \ln \frac{D_{r b}}{D_{r a}} \quad \text { Ec. } 5
$$

El ángulo de fase se corresponde con el ángulo de fase de la corriente que produce el flujo, ver Ec. 6.

$$
\begin{array}{ll}
\theta_{\Phi_{\text {rab }}}=\theta_{I_{r}} & \text { Ec. } 6
\end{array}
$$

\subsubsection{Tensión inducida en el Lazo "U"}

En primer lugar, se calcula el módulo de la Tensión “ $U_{\text {rab”, }}$ esto se presenta en la Ec. 7.

$U_{r a b}=\omega \times \Phi_{r a b}$

Ec. 7

El ángulo de fase de la tensión estará $90^{\circ}$ atrasado con respecto al del flujo, por lo que la fase de la tensión inducida estará dada por la Ec. 8.

$\theta_{U_{r a b}}=\theta_{\Phi_{r a b}}-90^{\circ}$

Ec. 8

\subsubsection{Inductancia propia del Lazo "L"}

La inductancia propia del lazo está dada por la Ec. 9.

$L=\frac{\mu_{0}}{\pi} \ln \left(\frac{\left|X_{a}-X_{b}\right|}{r m g}\right)=4 \times 10^{-7} \ln \left(\frac{\left|X_{a}-X_{b}\right|}{r m g}\right)$

Ec. 9

donde:

l: longitud del lazo

rmg: radio medio geométrico del conductor del lazo

\subsubsection{Reactancia inductiva del Lazo " $X_{L}$ "}

A partir de la inductancia propia del lazo está dada por la Ec. 9, es posible expresar la reactancia inductiva del lazo. Esto se presenta en la Ec. 10

$$
X_{L}=\omega \frac{\mu_{0}}{\pi} l \ln \left(\frac{\left|X_{a}-X_{b}\right|}{r m g}\right)=\omega 4 \times 10^{-7} l \ln \left(\frac{\left|X_{a}-X_{b}\right|}{r m g}\right)
$$

\subsubsection{Resistencia del Lazo "R"}

En general la resistencia por unidad de longitud ( $\mathrm{R}^{\prime}$ ) de los conductores es un dato brindado por los fabricantes y está expresada en $\Omega / \mathrm{km}$, en general referida a $20^{\circ} \mathrm{C}$. Para conocer la resistencia de cada lazo es necesario conocer la longitud total del conductor, y eventualmente la temperatura de trabajo. Por medio de la Ec. 11 es posible obtener la resistencia del lazo analizado.

$$
R=R^{\prime} l
$$

Ec. 11 
donde:

$\mathrm{R}$ : resistencia del conductor

R': resistencia por unidad de longitud

l: longitud del conductor

\subsubsection{Inductancia mutua entre un conductor de fase y un Lazo "LM $\mathrm{CL}_{\text {" }}$}

Si se considera la Ec. 9, es posible definir la inductancia mutua entre un conductor de fase un lazo pasivo "LM $\mathrm{CL}_{\text {", }}$ si se divide a la mencionada ecuación por la corriente del conductor " $\mathrm{I}_{\mathrm{r}}$ ", se obtiene la Ec. 12.

$$
L M_{C L}=\frac{\Phi_{r a b}}{I_{r}}=l \frac{\mu_{0}}{2 \pi} \ln \frac{D_{r b}}{D_{r a}}=2 \times 10^{-7} l \ln \frac{D_{r b}}{D_{r a}}
$$

\subsubsection{Reactancia inductiva conductor - lazo "X $\mathrm{X}_{\mathrm{LMCL}}$ "}

Si se considera ahora la Ec. 7 y la Ec. 12, se puede definir la reactancia inductiva entre un conductor de fase y un lazo pasivo. Esto se expresa en la Ec. 13

$$
X_{L M C L}=\omega \times 2 \times 10^{-7} l \ln \frac{D_{r b}}{D_{r a}}
$$

\subsubsection{Impedancia del Lazo "Z"}

Considerando los parámetros descriptos previamente, es posible determinar el módulo de la impedancia "Z", esto se observa en la Ec. 14.

$$
Z=\sqrt{R^{2}+(\omega L)^{2}}
$$

El ángulo correspondiente se presenta en la Ec. 15.

$$
\theta_{Z}=\operatorname{arctg} \frac{\omega L}{R}
$$

\subsubsection{Corriente del Lazo "I"}

Para estimar la corriente que circulará por el lazo se debe considerar la tensión inducida y la impedancia del lazo de la forma que se presenta en la Ec. 16.

$$
I_{r}=\frac{U_{r}}{Z}
$$

Se debe considerar a esta corriente con su módulo y fase. Hasta aquí se consideró la corriente que circulará debido a un solo conductor. En los casos planteados se consideran sistemas trifásicos, por lo que es necesario calcular las tensiones inducidas por cada fase, y a partir de ellas realizar la superposición y obtener la corriente resultante.

Cuando se realiza dicha superposición debe considerarse que son fasores, y realizar la correspondiente suma fasorial.

Esquemáticamente esto puede apreciarse en la Fig. 60, en donde para un sistema cuya secuencia es "RST (Directo)". Si la fase central, en este caso "S", se sitúa en el eje de la espira, esta no aporta al flujo total, por lo que las fases "R y T" son las que realizan los aportes. En estas condiciones el flujo total ( $\Phi_{\text {Total }}$ ) está dado por los flujos parciales $\left(\Phi_{\mathrm{R}} \mathrm{y} \Phi_{\mathrm{T}}\right)$. 
A $90^{\circ}$ de flujo total se ubica la tensión inducida ( $U_{\text {Inducida }}$ ) y atrasada con respecto a esta tensión la corriente inducida ( $\mathrm{I}_{\text {Inducida}}$ ). Hay que destacar que el ángulo final de esta corriente depende del ángulo de la impedancia del lazo considerado. Si dicho ángulo fuese cero, la corriente estaría en fase con la tensión.

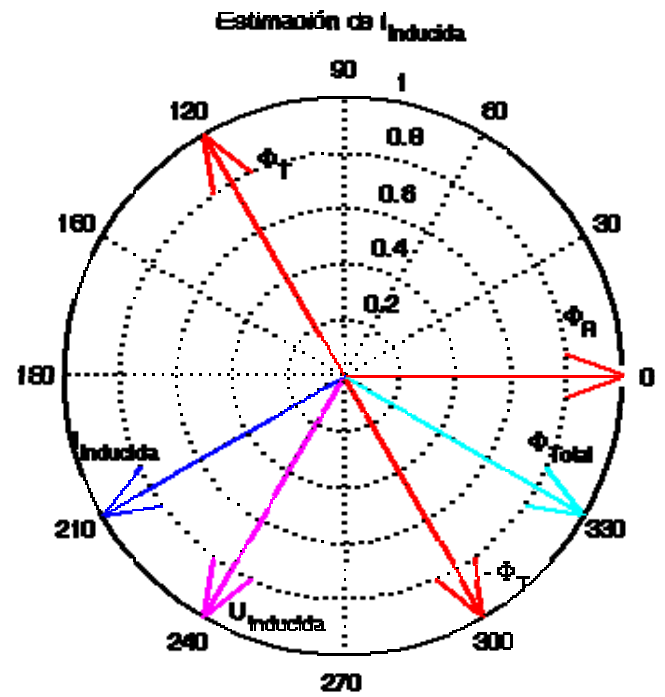

Fig. 60 - Diagrama fasorial para determinar "I Inducida".

En la Fig. 61 se aprecian los diferentes flujos magnéticos que intervienen, "Bo" y "Bi". Además se define una zona de interés, allí se realiza la superposición de los campos y se obtiene el resultante "Br".

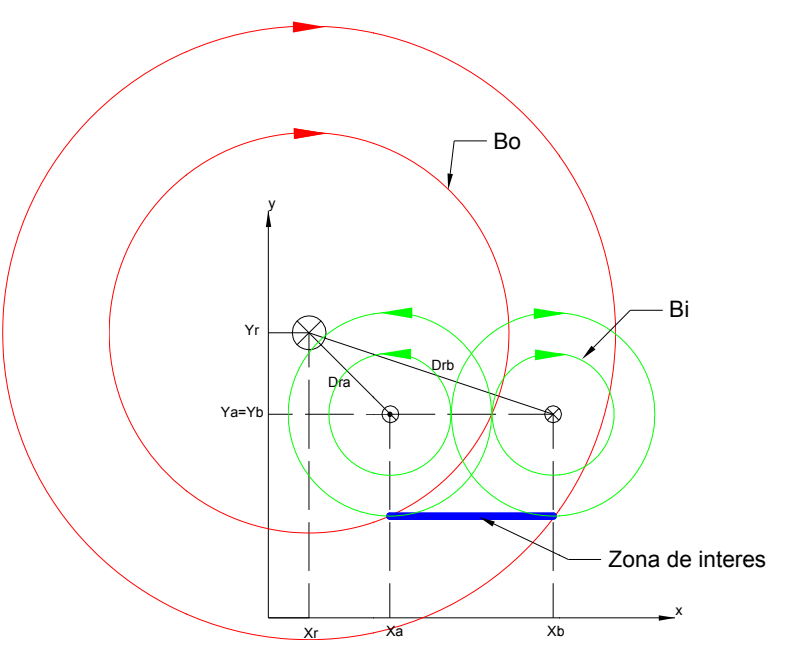

Fig. 61 - Flujos debido al campo original "Bo" y a la espira "Bi”.

En términos de ecuaciones es posible expresar el campo en la zona de interés de acuerdo a la Ec. 17, es necesario recordar que como se trata de una magnitud vectorial, debe realizarse la sumatoria vectorial.

$\overrightarrow{B_{r}}=\overrightarrow{B_{0}}+\overrightarrow{B_{i}}$

Ec. 17 


\subsubsection{Compensación}

En algunos casos es posible compensar la inductancia de los lazos de mitigación, para ello se utilizan capacitores dispuestos en serie con el lazo, para estos casos es necesario considerar en el cálculo de impedancia del lazo el efecto de esta reactancia capacitiva.

La inserción de un capacitor, reduce la componente inductiva del lazo, incrementando la corriente inducida en él, pero además modifica el ángulo de fase de la corriente por el lazo, en el caso de las configuraciones de lazo coplanar, deja de estar en oposición espacial con el creado por la línea. Este efecto provoca que no resulte de interés compensar totalmente la reactancia inductiva. La mayor importancia relativa de la componente resistiva de la impedancia del lazo, provoca que el tipo de conductor utilizado sea un parámetro a tener en cuenta. [31] [ 34].

Una adecuada selección del capacitor puede arrojar como resultado un incremento en el efecto de mitigación.

\subsection{Utilización de múltiples lazos pasivos}

En algunas circunstancias será necesario utilizar más de un lazo pasivo, para lo que debe considerarse la presencia de los demás lazos. Para ponderar la influencia de estos, debe considerarse además la inductancia mutua existente entre los diferentes lazos.

\subsubsection{Inductancia mutua entre dos lazos largos}

Para el cálculo de la impedancia mutua entre dos lazos, se deben consideran las hipótesis planteadas en 7.1.1. Esto permite reducir el problema a dos dimensiones.

En la Fig. 62 se aprecian dos lazos pasivos, uno de color rojo y el otro azul. En la Fig. 63 se presenta un corte transversal, en donde se aprecian las corrientes consideradas y las distancias entre los conductores.

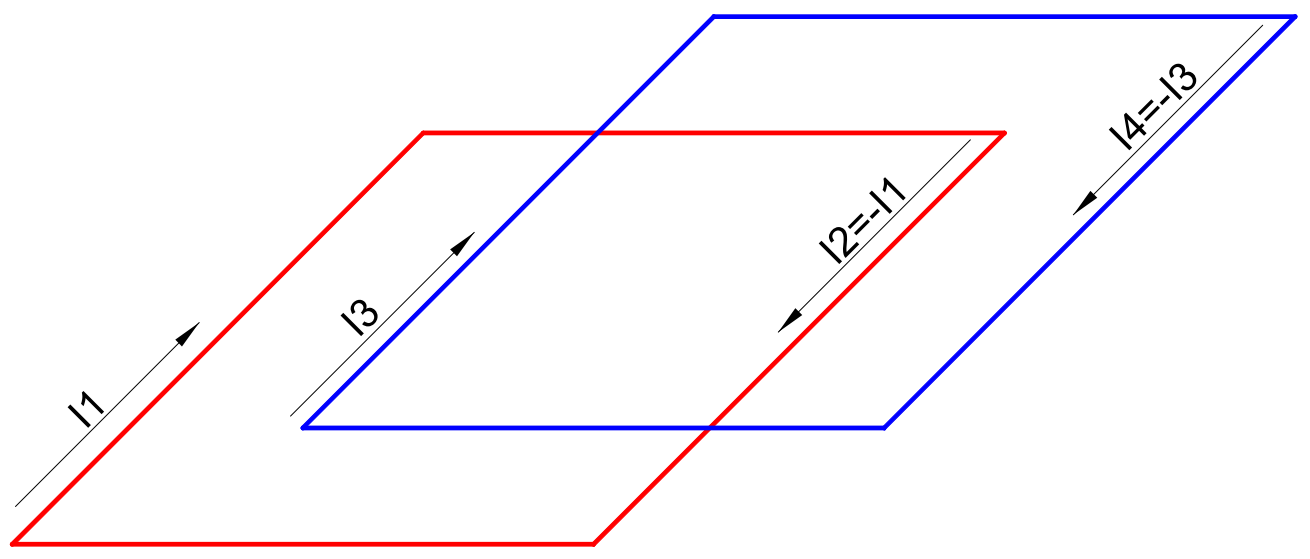

Fig. 62 - Conductores que forman dos lazos pasivos.

En primer lugar se determina el flujo que atraviesa el lazo "3-4", generado por la corriente por el conductor " 1 " $\left(\mathrm{I}_{1}\right)$. Si se considera la Ec. 5, es posible expresar el flujo al problema aquí planteado, Ec. 18.

$$
\Phi_{1(3-4)}=l \int_{D_{13}}^{D_{14}} B_{1} d r=l \int_{D_{13}}^{D_{14}} \frac{\mu_{0} I_{1}}{2 \pi r} d r=l \frac{\mu_{0} I_{1}}{2 \pi} \ln \frac{D_{14}}{D_{13}}=2 \times 10^{-7} I_{1} l \ln \frac{D_{14}}{D_{13}}
$$

El flujo concatenado en "3-4", debido a la corriente por el conductor "2", estará dado por la Ec. 19. 


$$
\Phi_{2(3-4)}=l \int_{D_{23}}^{D_{24}} B_{2} d r=l \int_{D_{23}}^{D_{24}} \frac{\mu_{0} I_{2}}{2 \pi r} d r=l \frac{\mu_{0} I_{2}}{2 \pi} \ln \frac{D_{24}}{D_{23}}=2 \times 10^{-7} I_{2} l \ln \frac{D_{24}}{D_{23}}
$$

Considerando que " $\mathrm{I}_{2}=-\mathrm{I}_{1}$ ", es posible calcular el flujo total a través de la espira " $3-4$ " (Ec. 20).

$$
\Phi_{12(3-4)}=2 \times 10^{-7} I_{1} l \ln \frac{D_{14}}{D_{13}}+2 \times 10^{-7} I_{2} l \ln \frac{D_{24}}{D_{23}}=2 \times 10^{-7} I_{1} l\left(\ln \frac{D_{14}}{D_{13}}-\ln \frac{D_{24}}{D_{23}}\right) \quad \text { Ec. } 20
$$

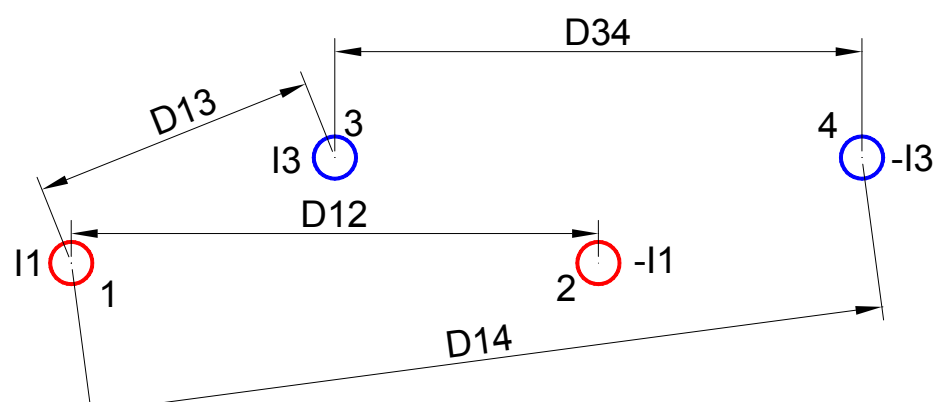

Fig. 63 - Conductores que forman dos lazos pasivos, corte transversal.

La inductancia mutua entre los lazos 12-34, estará dada por la Ec. 21.

$M_{(1-2)(3-4)}=\frac{\Phi_{12(3-4)}}{I_{1}}=\frac{2 \times 10^{-7} I_{1} l\left(\ln \frac{D_{14}}{D_{13}}-\ln \frac{D_{24}}{D_{23}}\right)}{I_{1}}=2 \times 10^{-7} l \ln \frac{D_{14} D_{23}}{D_{13} D_{24}}$

Ec. 21

\subsubsection{Inductancia mutua entre dos lazos cortos}

Se consideran lazos cortos a aquellos en que la longitud y el ancho del mismo son comparables; o bien los tramos paralelos a los conductores de fase son del mismo orden de magnitud que los de los tramos perpendiculares. Para determinar el flujo concatenado se debe recurrir a métodos numéricos de cálculo.

\subsubsection{Corriente Inducida en los lazos - Utilización de múltiples lazos}

En estas circunstancias, se debe considerar la influencia de todos los conductores que intervienen en el sistema y sus respectivas corrientes. Además hay que considerar las impedancias propias de los conductores de lazo, las impedancias mutuas entre los diferentes lazos y las impedancias mutuas entre conductores de fases y lazos.

Considerando las magnitudes mencionadas previamente, un sistema formado por $\mathrm{N}$ lazos pasivos y M conductores de fase, para un lazo (Lazo 1) es posible expresar la Ec. 22.

$0=I_{1} Z_{11}+I_{2} Z_{12}+\cdots+I_{N} Z_{1 N}+I_{f 1} Z_{1 f 1}+I_{f 2} Z_{1 f 2}+\cdots+I_{f M} Z_{1 f M}$

Ec. 22

Donde:

$\mathrm{Z}_{11}$ : es la impedancia propia del lazo 1.

$\mathrm{Z}_{1 \mathrm{~N}}$ : es la impedancia mutua entre el lazo 1 y el lazo $\mathrm{N}$.

$\mathrm{Z}_{1 \mathrm{fm}}$ : es la impedancia mutua entre el lazo 1 y el conductor de fase $\mathrm{M}$.

$\mathrm{I}_{1}$ : corriente por el conductor del lazo 1.

$\mathrm{I}_{\mathrm{N}}$ : corriente por el conductor del lazo $\mathrm{N}$. 
$\mathrm{I}_{\mathrm{fM}}$ : corriente el conductor de la fase $\mathrm{M}$.

Analizando la Ec. 22, se observa que los términos correspondientes a las corrientes de fase son conocidos. Los demás dependen de las corrientes de lazo, que son incógnitas. Para resolver este problema hay que plantear un sistema de ecuaciones que permita encontrar la solución.

Si se reordena la Ec. 22, de la forma presentada en la Ec. 23, los términos que son conocidos quedan de un lado de la igualdad.

$-\left(I_{f 1} Z_{1 f 1}+I_{f 2} Z_{1 f 2}+\cdots+I_{f M} Z_{1 f M}\right)=I_{1} Z_{11}+I_{2} Z_{12}+\cdots+I_{N} Z_{1 N}$

Ec. 23

A la derecha de la igualdad se observa en primer término la influencia de la impedancia propia del lazo "1" y la respectiva corriente, los otros términos dan cuenta de la impedancia mutua entre lazos, con las respectivas corrientes.

Si ahora a los términos correspondientes a las corrientes de fase y las respectivas impedancias se los renombra como se presenta en la Ec. 24, es posible expresar a la Ec. 23 de la forma presentada en la Ec. 25.

$$
\begin{aligned}
& -\left(I_{f 1} Z_{1 f 1}+I_{f 2} Z_{1 f 2}+\cdots+I_{f M} Z_{1 f M}\right)=U_{1} \\
& U_{1}=I_{1} Z_{11}+I_{2} Z_{12}+\cdots+I_{N} Z_{1 N}
\end{aligned}
$$

Considerando la Ec. 25 y contemplando que existen "N" lazos pasivos, es necesario evaluar la influencia de todos los lazos, esto se logra mediante la Ec. 26.

$$
\left[\begin{array}{c}
\dot{U}_{1} \\
\dot{U}_{2} \\
\dot{U}_{3} \\
\square \\
\dot{U}_{N}
\end{array}\right]=\left[\begin{array}{ccccc}
Z_{l 1} & 0 & 0 & 0 & 0 \\
0 & Z_{l 2} & 0 & 0 & 0 \\
0 & 0 & Z_{l 2} & 0 & 0 \\
0 & 0 & 0 & \square & 0 \\
0 & 0 & 0 & 0 & Z_{l N}
\end{array}\right]\left[\begin{array}{c}
\dot{I}_{1} \\
\dot{I}_{2} \\
\dot{I}_{3} \\
\square \\
\dot{I}_{N}
\end{array}\right]+\left[\begin{array}{ccccc}
0 & j X_{m 12} & j X_{m 13} & \square & j X_{m 1 N} \\
j X_{m 21} & 0 & j X_{m 23} & \square & j X_{m 2 N} \\
j X_{m 31} & j X_{m 32} & 0 & \square & j X_{m 3 N} \\
\square & \square & \square & 0 & \square \\
j X_{m N 1} & j X_{m N 2} & j X_{m N 3} & \square & 0
\end{array}\right]\left[\begin{array}{c}
\dot{I}_{1} \\
\dot{I}_{2} \\
\dot{I}_{3} \\
\square \\
\dot{I}_{N}
\end{array}\right]
$$

Donde:

$\mathrm{U}_{\mathrm{i}}$ : es la tensión inducida en el lazo “i”, por los todos los conductores de fase.

$\mathrm{Z}_{\mathrm{li}}$ : es la impedancia propia del lazo "i"

$\mathrm{X}_{\mathrm{miN}}$ : es la reactancia inductiva mutua entre el lazo "i" y el lazo "N"

Esto se puede expresar de una forma más compacta agrupando las matrices de impedancia propia y mutua, esto se presenta en la Ec. 27.

$$
\left[\begin{array}{c}
\dot{U}_{1} \\
\dot{U}_{2} \\
\dot{U}_{3} \\
\square \\
\dot{U}_{N}
\end{array}\right]=\left[\begin{array}{ccccc}
Z_{l 1} & j X_{m 12} & j X_{m 13} & \square & j X_{m 1 N} \\
j X_{m 21} & Z_{l 2} & j X_{m 23} & \square & j X_{m 2 N} \\
j X_{m 31} & j X_{m 32} & Z_{l 3} & \square & j X_{m 3 N} \\
\square & \square & \square & \square & \square \\
j X_{m N 1} & j X_{m N 2} & j X_{m N 3} & \square & Z_{l N}
\end{array}\right]\left[\begin{array}{c}
\dot{I}_{1} \\
\dot{I}_{2} \\
\dot{I}_{3} \\
\square \\
\dot{I}_{N}
\end{array}\right]
$$

Para obtener las corrientes por los lazos, es necesario despejar el vector de corrientes de la Ec. 27 y resolver el sistema de ecuaciones. Esto vendrá dado por la Ec. 28. 


$$
\left[\begin{array}{c}
\dot{I}_{1} \\
\dot{I}_{2} \\
\dot{I}_{3} \\
\square \\
\dot{I}_{N}
\end{array}\right]=\left[\begin{array}{ccccc}
Z_{l 1} & j X_{m 12} & j X_{m 13} & \square & j X_{m 1 N} \\
j X_{m 21} & Z_{l 2} & j X_{m 23} & \square & j X_{m 2 N} \\
j X_{m 31} & j X_{m 32} & Z_{l 3} & \square & j X_{m 3 N} \\
\square & \square & \square & \square & \square \\
j X_{m N 1} & j X_{m N 2} & j X_{m N 3} & \square & Z_{l N}
\end{array}\right]^{-1}\left[\begin{array}{c}
\dot{U}_{1} \\
\dot{U}_{2} \\
\dot{U}_{3} \\
\square \\
\dot{U}_{N}
\end{array}\right]
$$

A partir de aquí se desarrolla una herramienta de cálculo que permite resolver el sistema de la Ec. 28. De esta manera se puede cuantificar la influencia en los lazos pasivos de los conductores de fase y de las corrientes por todos los lazos.

\subsection{Desarrollo de herramientas de cálculo}

Con el objeto de atender los requisitos de la normativa, antes de construir nuevas instalaciones es necesario evaluar si estas cumplirían con los mismos. Para lo cual resulta necesario contar con herramientas que permitan cuantificar los valores de campo magnético emitidos por las instalaciones proyectadas. Para llevar adelante esta tarea se decidió desarrollar herramientas que permitan realizar este tipo de evaluaciones.

Se trabajó en la elaboración de un software que permita cubrir las necesidades mencionadas, pero que además permita evaluar la implementación de lazos pasivos para lograr reducir los niveles de campo magnético generado por instalaciones que admitan esta solución. Como resultado surge el desarrollo de la herramienta de cálculo que se describe en esta tesis.

Con el planteo matemático del problema resuelto, se llevó a cabo la implementación del software, luego se realizaron mediciones en instalaciones montadas en laboratorio que permitieran validar los resultados arrojados por el programa de cálculo. Cuando todos los pasos fueron cumplimentados se verificó el desempeño del mismo en instalaciones existentes en la República Argentina, para lo cual se realizaron cálculos y mediciones, que fueron luego comparadas [ 39 ].

\subsubsection{Software}

El software desarrollado es aplicable a líneas de transmisión de energía eléctrica (aéreas o cables subterráneos), en donde se considera que los conductores son muy largos. Con esta consideración sólo se tendrán componentes de campo magnético en el plano perpendicular a la dirección de las corrientes.

El software se dividió en dos partes, una para realizar el cálculo de campo magnético y otra para la implementación de los lazos pasivos. El programa se desarrolló en el entorno de Matlab.

El módulo de cálculo de campo magnético define el valor que se obtiene ante la configuración de " $n$ " conductores, dispuestos geométricamente, con valores individuales de corrientes (módulos y fase). Este módulo se utiliza cuando sólo se consideran los conductores de fase y cuando también son incorporados los lazos.

El módulo para la implementación de lazos pasivos, calcula la corriente inducida en los mismos ante la presencia de los conductores de fase. Se requieren los datos y ubicaciones de los conductores de fase y de los lazos y las corrientes de los conductores de fase.

En la Fig. 64 se presenta un diagrama de flujo con el esquema de utilización de los módulos desarrollados. 


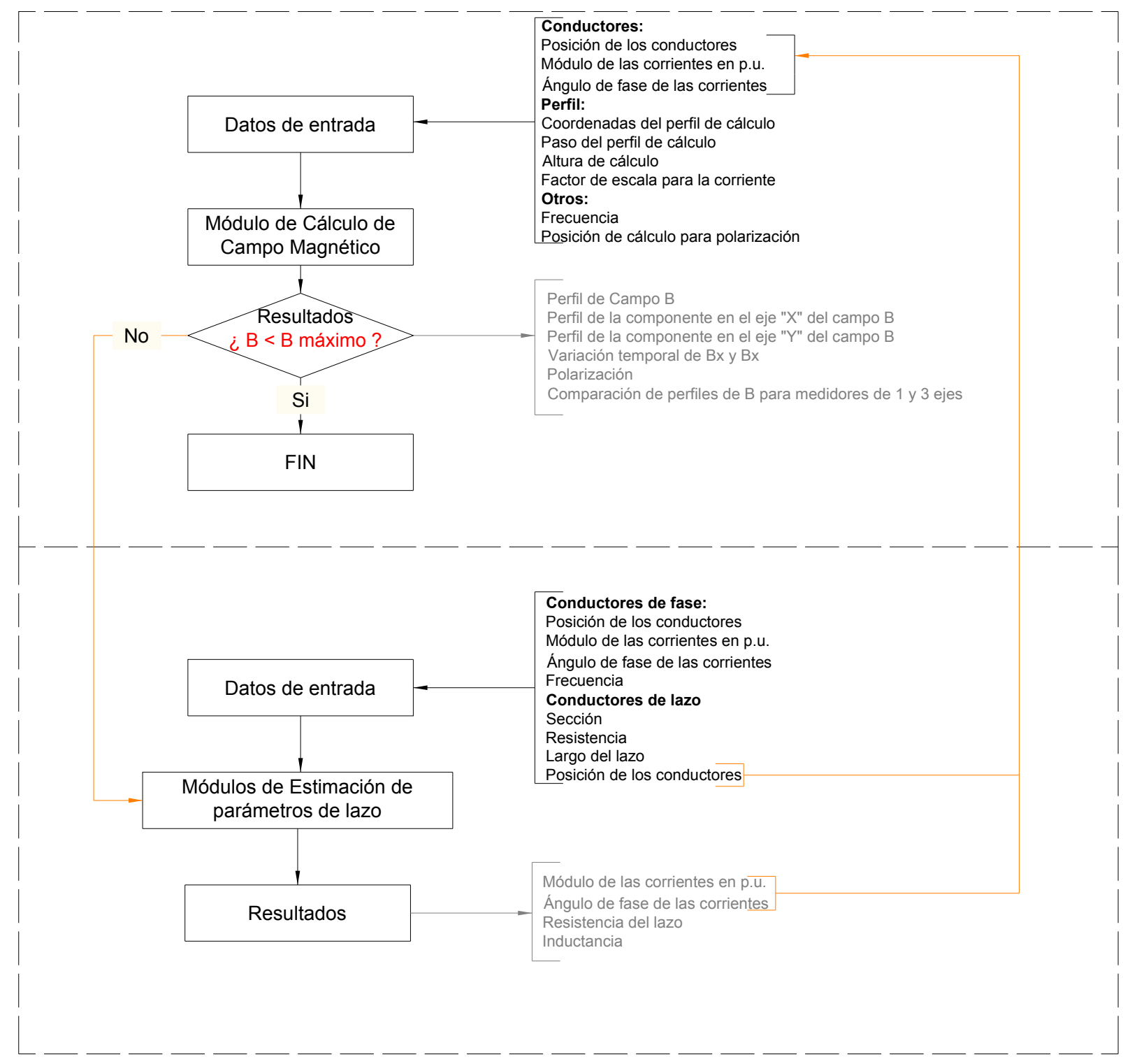

Fig. 64 - Esquema de utilización de las herramientas de cálculo

En los siguientes incisos se describen las principales características de cada módulo.

\subsubsection{Módulo de cállculo de Campo Magnétíco -2D}

En esta etapa se realiza la estimación de los valores de campo magnético, a partir de las posiciones relativas de los conductores que intervienen en cada problema y las corrientes asociadas a los mismos, en módulo (p.u.) y fase. Estos datos se ingresan en un archivo "*.txt".

En la interfaz gráfica, Fig. 65, entre el usuario y el programa, se pueden encontrar varias opciones, entre las que se mencionan algunos datos de entradas como ser: inicio, fin y paso del perfil en donde se realiza el cálculo; altura de cálculo, el valor cero corresponde al nivel del suelo, y valor de corriente. Esto se puede apreciar en la Fig. 65 a). En el caso de requerirse corrientes desbalanceadas en módulo y fase, estos deben introducirse en p.u. en el documento “*.txt”.

Por otra parte, se deben seleccionar cuales serán los resultados requeridos Fig. 65 b), las posibilidades son:

- Perfil del módulo de campo magnético 
- Perfil de las componentes en "x" o " $y$ "

- Variación temporal de Bx, By y el Módulo de B

- Polarización del campo, en un punto que debe ser seleccionado en "Posición de la Elipse"

- Comparación entre los perfiles que se obtendrían utilizando un instrumento de medición de un eje y uno de tres ejes

El algoritmo de cálculo sigue el procedimiento descripto por las ecuaciones presentadas en AII-11 (Campo Magnético generado por un sistema de "n" conductores paralelos).

Para los cálculos de campo magnético se realizan modelos bidimensionales y se calculan los valores de campo en cada eje, con estos valores se procede a obtener el campo resultante.

Los resultados se presentan por medio de perfiles pero también se incluye la indicación numérica del valor máximo de $B_{R}$ para el perfil analizado " $B_{\max }$ ".

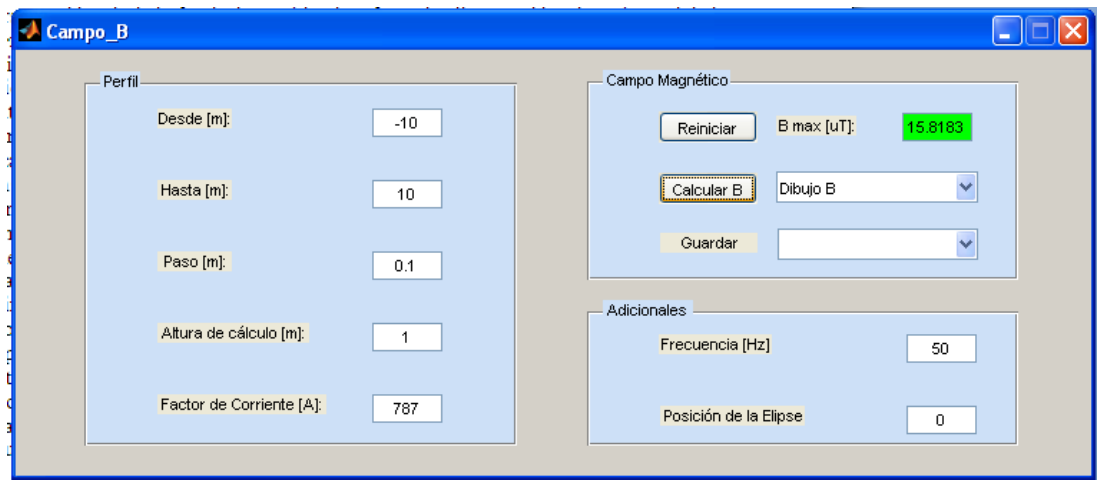

a) Interfaz general

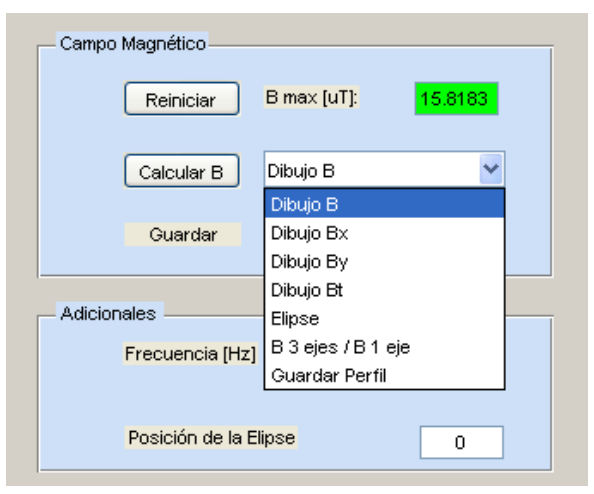

b) Detalle opciones de cálculo

Fig. 65 - Interfaz gráfica del módulo de cálculo de campo magnético

Anteriormente se mencionó que es posible obtener la polarización del campo magnético. Para estos fines se deben ingresar datos adicionales, como la posición en donde se desea conocer la polarización.

Otra opción disponible es exportar los resultados obtenidos, esto se lleva a cabo exportando los valores obtenidos a archivos “*.txt". Entre las opciones se presenta el perfil de campo magnético resultante $B_{R}$ en función de las coordenadas, las componentes $B_{X} y$ BY en función de las coordenadas y los valores $\mathrm{B}_{\mathrm{X}}-\mathrm{B}_{\mathrm{Y}}$.

Los resultados obtenidos se representan en gráficos que se muestran en la Fig. 66.

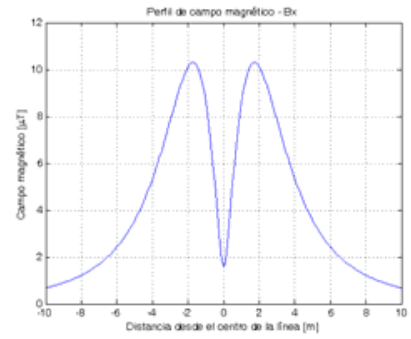

a) Perfil de "Bx"

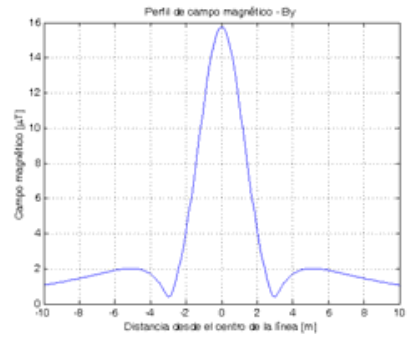

b) Perfil de "BY"

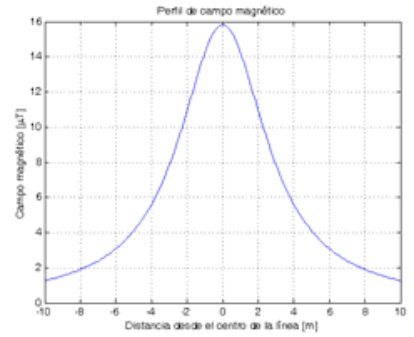

c) Perfil de "BR" 


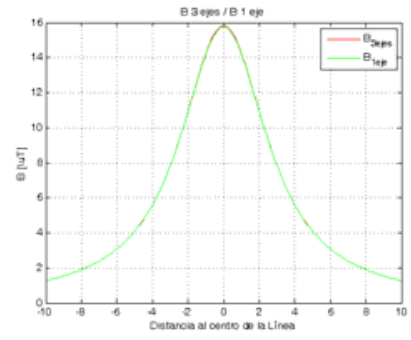

d) Comparación $B_{3 \text { ejes }} / B_{1 \text { eje }}$

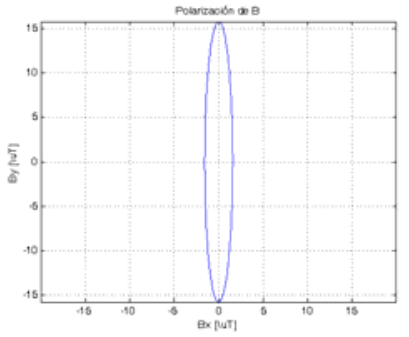

e) Polarización

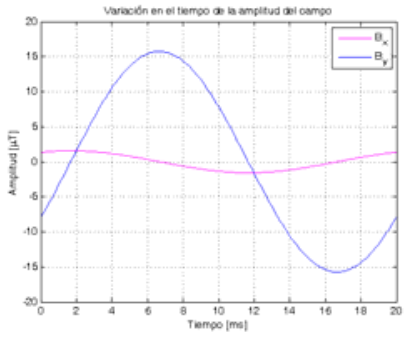

f) Variación temporal de $B_{X}$ y $B_{Y}$

Fig. 66 - Resultados obtenidos con el programa.

Es importante destacar que los medidores de campo de 3 ejes son bastante costosos si se los compara con los de 1 eje, por esta razón la utilización de medidores de 1 eje es muy común. Considerando esta situación, resulta de interés conocer cuál sería la diferencia entre las indicaciones de estos dos tipos de instrumentos. Para tal fin en la la Fig. 66 d), se presenta una comparación entre el perfil de campo magnético que se obtendría con los dos tipos de instrumentos.

\subsubsection{Módulo de estimación de parámetros de lazos - Lazo úníco}

Con este módulo es posible analizar el efecto de introducir un lazo pasivo en el entorno de una línea de transmisión de energía eléctrica. Se calcula la impedancia de los lazos y la corriente inducida en los mismos, debido a las corrientes que circulan por los conductores de fase de la línea.

La información de entrada se divide en dos grupos, por un lado lo que corresponde a los conductores de fase y por otro la información correspondiente al conductor del lazo.

Para los conductores de fase se deben ingresar: la frecuencia, corrientes de fase (en módulo y ángulo), posiciones relativas de los mismos (en coordenadas "x e y").

Para los lazos se requiere la posición relativa en " $\mathrm{x}$ e y", la longitud del lazo, la resistividad en $[\Omega / \mathrm{km}]$ y la sección del conductor considerado.

En la Fig. 67 se presenta la interfaz correspondiente a este módulo, allí pueden verse todas las posibilidades antes mencionadas. 


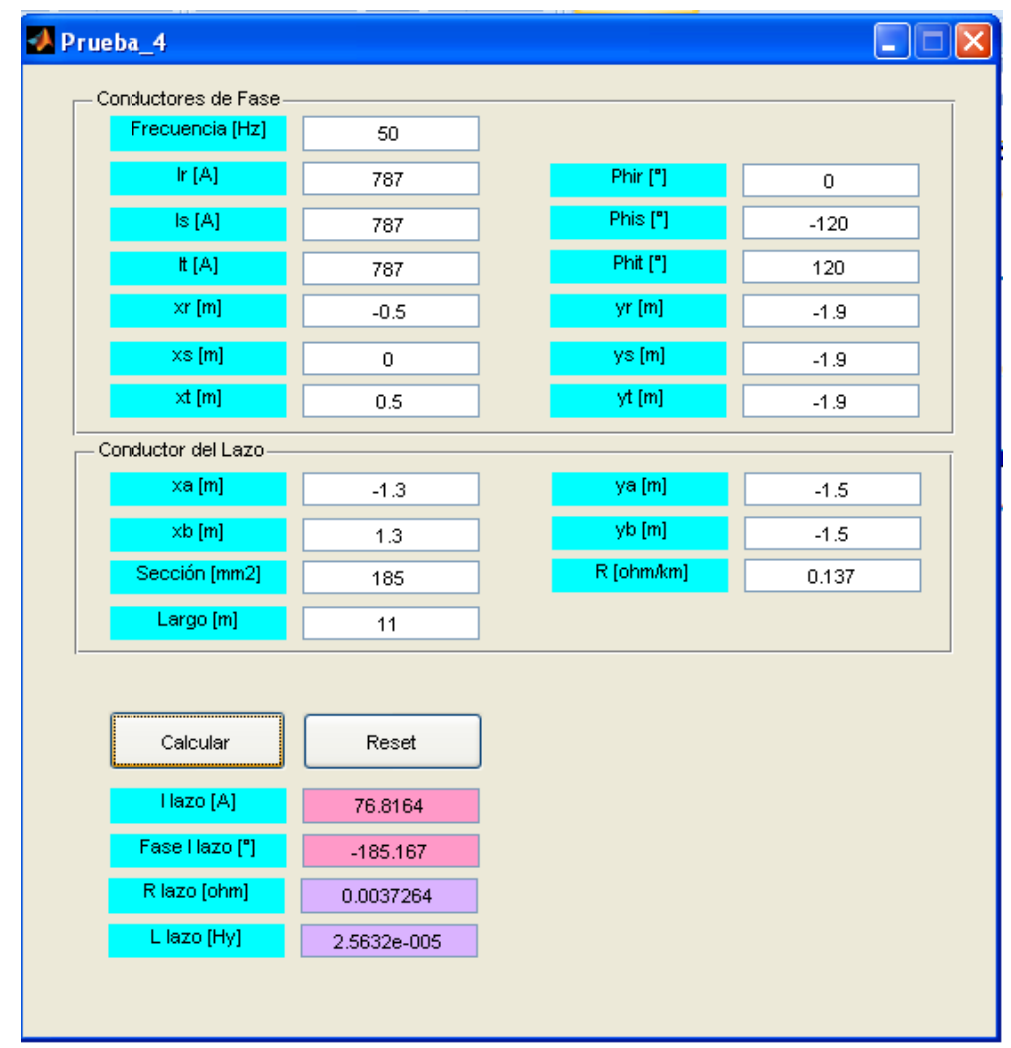

Fig. 67 - Interfaz gráfica, módulo de estimación de parámetros de lazo.

Una vez que toda la información es ingresada se obtiene la corriente que circulará por el lazo, en módulo y fase, pero también se obtiene la resistencia [ $\Omega$ ] y la inductancia del lazo [H]. La corriente es graficada en un diagrama fasorial en donde se compara con las corrientes de fase de la línea de transmisión.

Los resultados se indican en forma numérica y en forma gráfica, en la Fig. 68 se presenta la información de salida, en a) se aprecia la parte numérica y en b) la parte gráfica, en azul se muestran las corrientes de fase y en rojo la corriente inducida, esta se magnifica para que sea posible apreciarla en relación con las corrientes de fase.

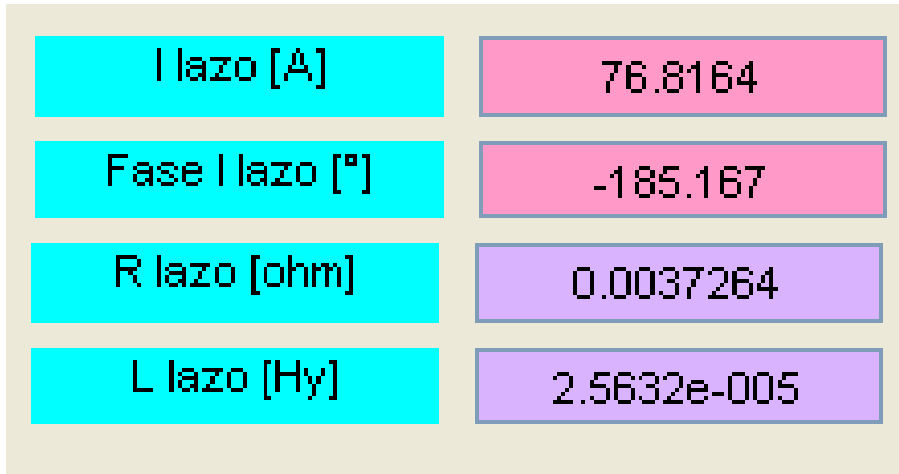

a) Valores numéricos

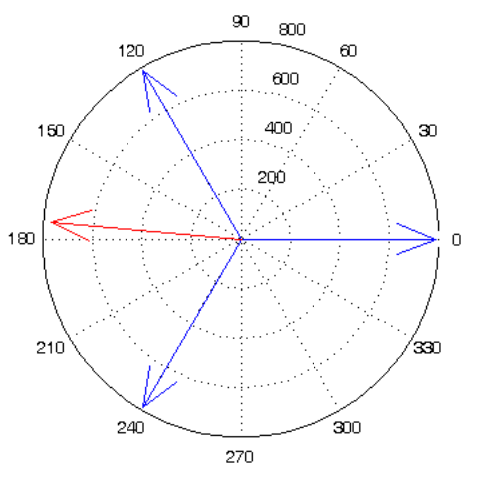

b) Diagrama fasorial de corrientes

Fig. 68 - Módulo de presentación de resultados, módulo de estimación de parámetros de lazo.

El algoritmo de cálculo resuelve las ecuaciones presentadas en los incisos 7.1.2 a 7.1.6. 


\subsubsection{Módulo de estimación de parámetros de lazos - Múltiples lazos}

Como se describió anteriormente, para estos casos es necesario resolver un sistema de ecuaciones que considere el efecto de la inductancia mutua entre los lazos. Para tal fin se llevó a cabo una herramienta adicional que contemple dicha situación.

Los datos de entrada son geométricos, se obtienen a partir de las posiciones de los lazos y se ingresan a partir de un archivo en formato "*.txt".

Por otro lado se ingresa el efecto de los conductores de fase sobre el lazo pasivo, este vector se obtiene a partir del módulo anterior.

El algoritmo de cálculo resuelve las ecuaciones presentadas en los incisos 7.1.2 a 7.1.6 además considera la inductancia mutua entre los diferentes lazos, este procedimiento se describe en los incisos 7.2.1 a 7.2.3.

En resumen, este módulo opera armando la matriz de impedancias de lazos y considerando el vector de tensiones, entrega como resultado un vector con valores de corriente en módulo y fase, en un archivo en formato “*.txt" compatible con el módulo de cálculo de campo magnético. Estos resultados pueden ser utilizados para realizar cálculos en 2D y 3D.

Para el cálculo del campo magnético, este vector de corrientes y las posiciones espaciales son utilizados en el módulo de campo magnético. A partir de aquí se pueden optar por dos opciones, realizar los cálculos en 2D, utilizando el módulo descripto en el inciso 7.3.1.1, o bien utilizarlo en cálculos 3D, para lo cual se dispone de una herramienta desarrollada en el entorno del programa Opera 10.5 de Vector Fields, siguiendo el procedimiento descripto en el punto 5.1 .

\subsection{Implementación práctica y Validación de modelos}

El proceso de cálculo descripto anteriormente y la herramienta desarrollada debieron ser verificados comparando los resultados obtenidos por medio de cálculos y mediciones. Para esto fue necesario implementar modelos a escala que permitan reproducir la mayor parte de los parámetros que intervienen en los procesos de cálculo [ 38 ].

Para lograr el objetivo se consideró un sistema trifásico como el de la Fig. 69, en donde se aprecian en un mismo plano los tres conductores de fase y un lazo pasivo.

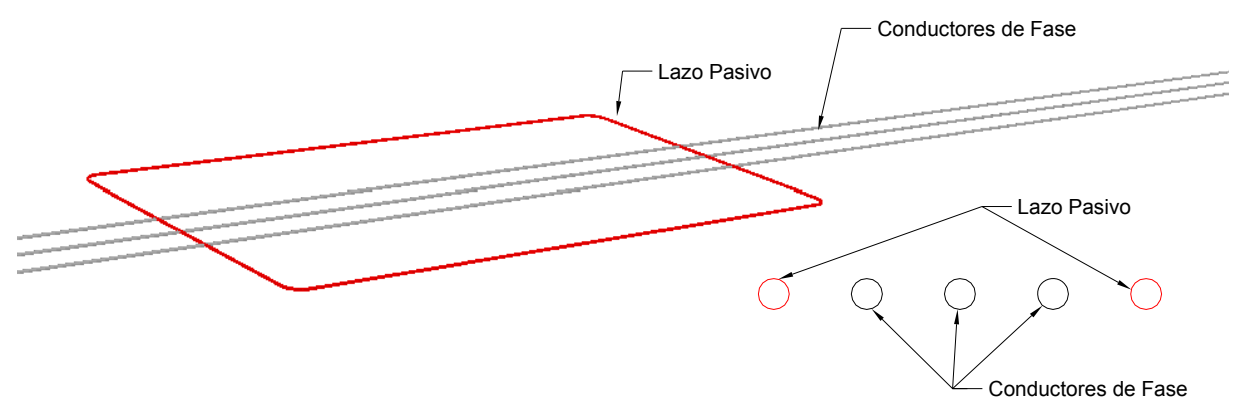

Fig. 69 - Arreglo de sistema trifásico con un lazo pasivo.

Tomando como referencia la fase "R", como resultado se obtiene el diagrama fasorial de la Fig. 60, en donde se representa el flujo concatenado con la espira originado por las fases "R"

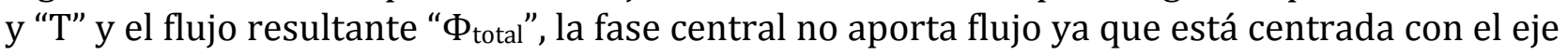
de la espira. A "-90" de $\Phi_{\text {total, }}$ se observa la tensión inducida "U inducida" y atrasada con respecto a esta se aprecia la corriente inducida "I". 
Esta metodología se utilizó para calcular las corrientes inducidas en el lazo para diferentes casos que se presentan en la Tabla IX, en donde se observan las características de los lazos utilizados. Para todos los casos se consideró una frecuencia de $50 \mathrm{~Hz}$.

La separación entre los conductores de fase en todos los casos considerados fue de 0,25 m. El caso "0" corresponde a la implementación de la línea sin la presencia de lazos pasivos.

Tabla IX - Casos considerados

\begin{tabular}{|c|c|c|c|c|}
\hline \multirow{2}{*}{ Caso } & \multicolumn{3}{|c|}{ Lazo } & \multirow{2}{*}{ Secuencia } \\
\cline { 2 - 4 } & Sec. & Ancho & Long & \\
\cline { 2 - 4 } & {$\left[\mathbf{m m}^{\mathbf{2}}\right]$} & {$[\mathbf{m}]$} & {$[\mathbf{m}]$} & \\
\hline 0 & \multicolumn{3}{|c|}{ Sin Lazo } & RST/RTS \\
\hline 1 & 35 & 1,0 & 2,85 & RST \\
\hline 2 & 35 & 1,0 & 2,85 & RTS \\
\hline 3 & 95 & 1,0 & 4,45 & RST \\
\hline 4 & 95 & 1,0 & 4,45 & RTS \\
\hline 5 & 95 & 1,5 & 4,00 & RST \\
\hline 6 & 95 & 0,5 & 4,75 & RST \\
\hline
\end{tabular}

\subsubsection{Medición de la Corriente Inducida}

En la Fig. 70 se presenta uno de los arreglos experimentales empleado para realizar las pruebas. Allí pueden verse los conductores de fase, uno de los lazos instalados y el sistema de alimentación, medición y registro. En todos los casos se consideró una configuración coplanar horizontal.

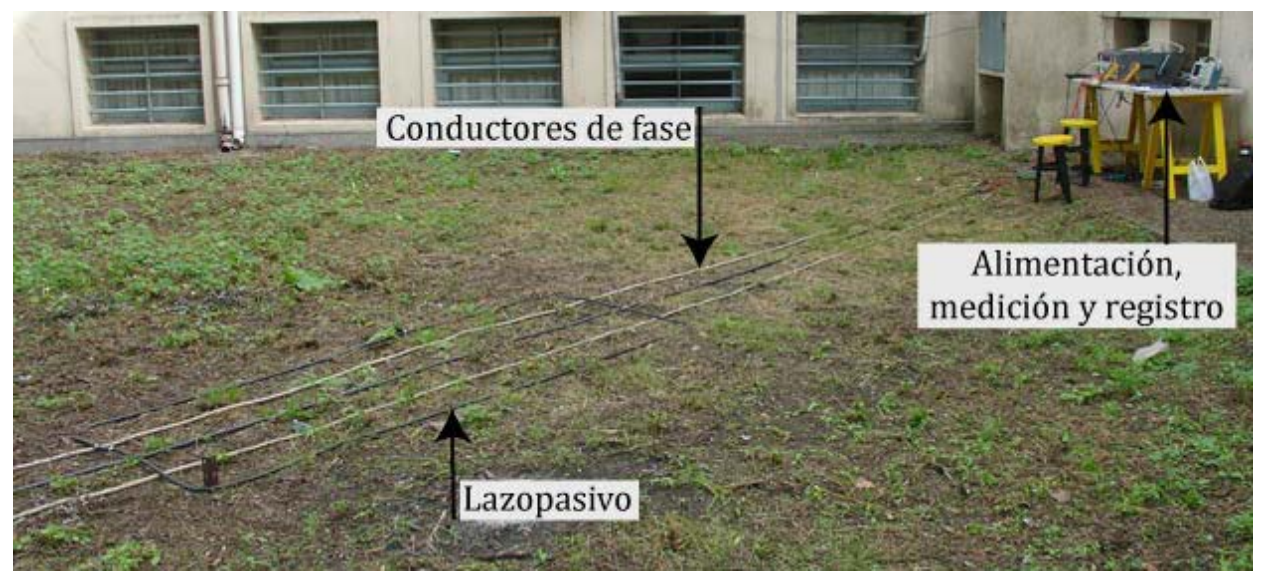

Fig. 70 - Arreglo para el circuito experimental, conductores de fase, lazo pasivo y elemento de medición.

En la Fig. 71, se aprecia un detalle de la zona de instalación del lazo pasivo, además se indica la zona de instalación de una pinza amperimétrica para la medición de la corriente inducida en el lazo. 


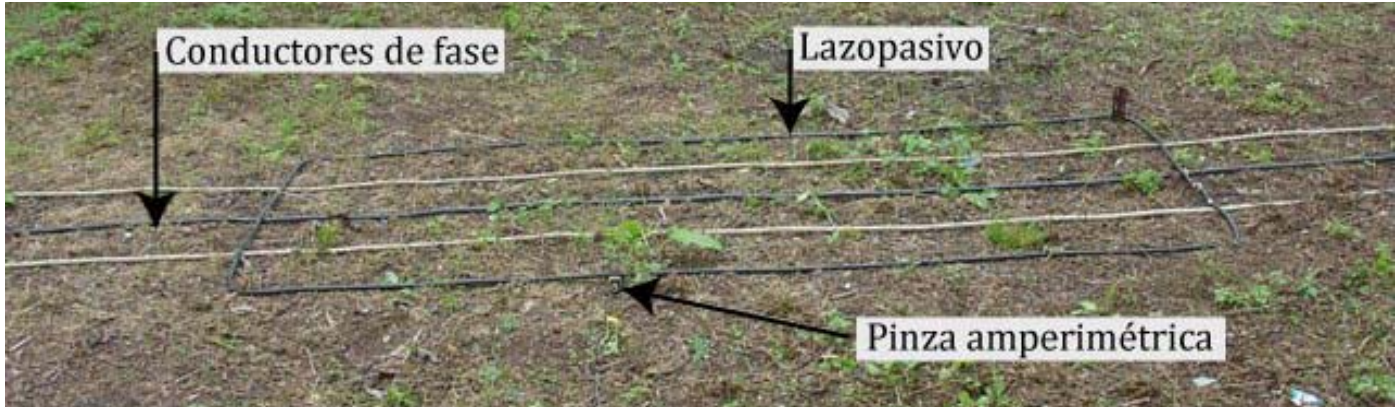

Fig. 71 - Arreglo para el circuito experimental, conductores de fase, lazo pasivo y elemento de medición.

Para la inyección de corriente se utilizó una fuente de corriente que posee 4 canales que entregan 30 A y 200 VA cada uno. Es posible ajustar el módulo y la fase de las corrientes en cada uno de los canales.

Para la medición de la corriente inducida en el lazo se utilizó una pinza cuyo rango máximo es de $5 \mathrm{~A}$ y posee un error de $1 \%$ entre 1 y $5 \mathrm{~A}$ y el error de fase es de $4^{\circ}$ entre $100 \mathrm{~mA}$ y $5 \mathrm{~A}$. Para la corriente de fase de referencia se utilizó una pinza cuyo rango es de $30 \mathrm{~A}$, que posee un error de $5 \%$ y el error de fase es de $5^{\circ}$. Las dos corrientes se introdujeron en un osciloscopio de cuatro canales, $100 \mathrm{MHz}$ y $1 \mathrm{GS} / \mathrm{s}$.

En la Fig. 72 se muestra la fuente utilizada, el osciloscopio empleado y otros elementos utilizados en las pruebas.

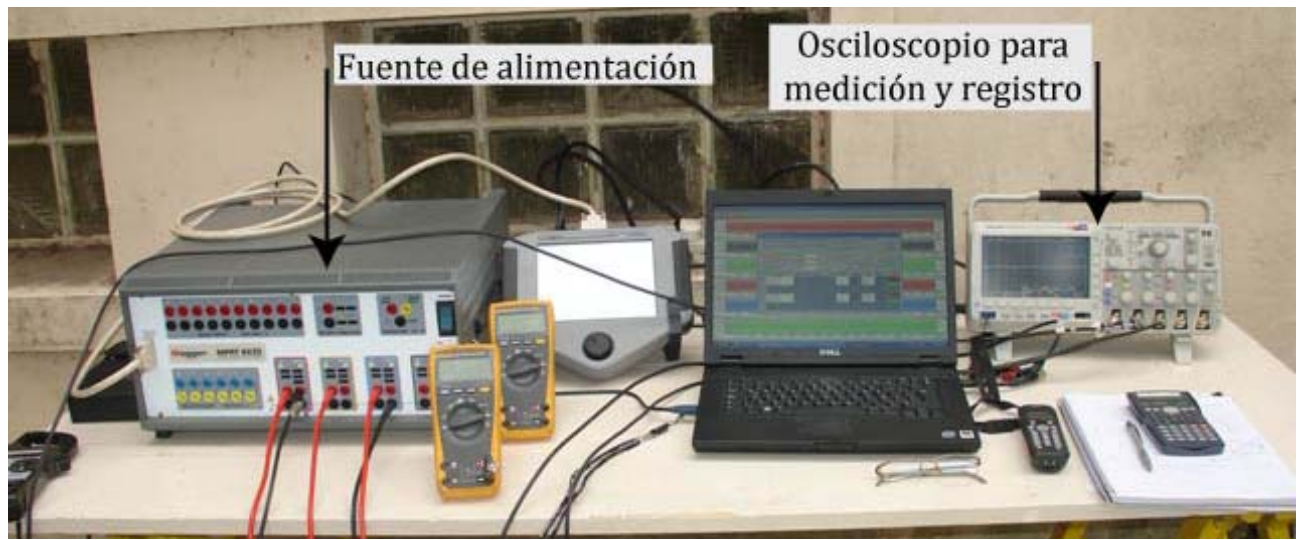

Fig. 72 - Arreglo para el circuito experimental, conductores de fase, lazo pasivo y elemento de medición.

Se registraron las corrientes en cada uno de los casos y se midió la amplitud y la diferencia de fase, tomando como referencia la corriente de fase "R". En la Fig. 73 se presenta uno de los registros obtenidos, se observa la corriente de fase de referencia, la corriente inducida en el lazo y se agregó la onda que representa la corriente calculada para el caso en que se utilizo conductores de $35 \mathrm{~mm}^{2}$ y secuencia RST. Las corrientes inducidas en el lazo se magnificaron para poder apreciarlas con la de referencia. 


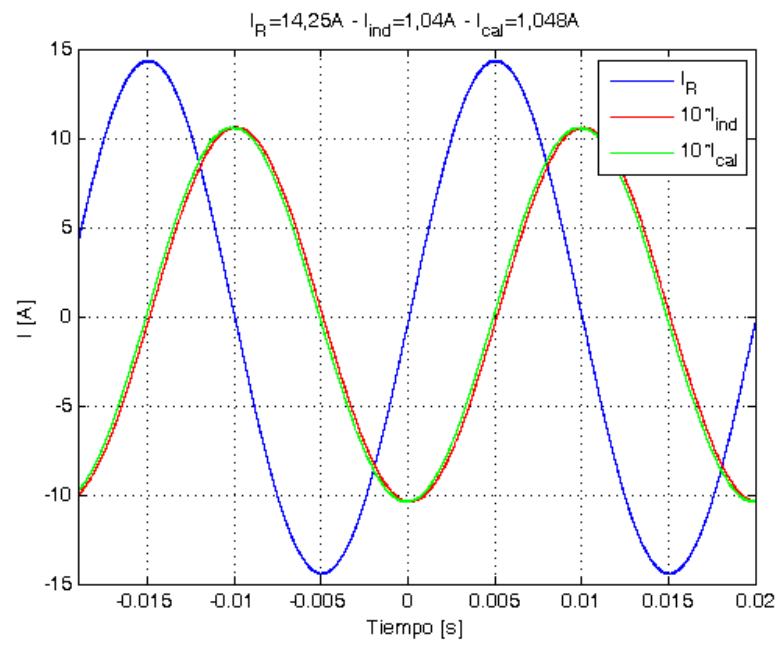

Fig. 73 - Corrientes de fase y corriente por lazo, calculada y medida para secuencia RST.

En la Tabla X se presentan los resultados de las corrientes medidas (M) y calculadas (C) para los casos "1 a 6". Además se incluyeron los errores existentes entre los valores calculados y los medidos, tomando como referencia el valor medido.

Tabla X - Comparación entre valores calculados y medidos de corriente

\begin{tabular}{|c|c|c|c|c|c|c|c|}
\hline \multirow{2}{*}{ Caso } & $\mathbf{I}_{\text {fase }}$ & \multicolumn{2}{|c|}{$\mathbf{I}_{\text {Lazo. C }}$} & \multicolumn{2}{c|}{$\mathbf{I}_{\text {Lazo M }}$} & \multicolumn{2}{c|}{ Errores } \\
\cline { 2 - 8 } & & Mod. & Fase & Mod. & Fase & Mod. & Fase \\
\cline { 2 - 8 } & {$[\mathbf{A}]$} & {$[\mathbf{A}]$} & {$\left[{ }^{\circ}\right]$} & {$[\mathbf{A}]$} & {$\left[{ }^{\circ}\right]$} & {$[\%]$} & {$[\%]$} \\
\hline 1 & 14,3 & 1,05 & -147 & 1,03 & -135 & 2 & 9 \\
\hline 2 & 14,3 & 1,05 & -86 & 1,04 & -90 & 1 & -4 \\
\hline 3 & 14,9 & 1,98 & -166 & 1,9 & -175 & 4 & -5 \\
\hline 4 & 14,9 & 1,98 & -107 & 1,9 & -116 & 4 & -8 \\
\hline 5 & 14,8 & 1,1 & -167 & 1,04 & -175 & 6 & -5 \\
\hline 6 & 14,9 & 1,1 & -106 & 1,06 & -114 & 4 & -7 \\
\hline
\end{tabular}

Los errores en el módulo de la corriente inducida en el lazo variaron entre 1 y $6 \%$. Los errores de fase fueron mayores, resultando el mayor igual a $9 \%$.

\subsubsection{Análisis de la Impedancia de Lazos Pasivos}

En la Tabla XI se presentan los resultados de los valores calculados para la impedancia en cada uno de los casos considerados.

Tabla XI - Valores calculados de impedancia a $50 \mathrm{~Hz}$

\begin{tabular}{|c|c|c|c|c|c|c|c|}
\hline \multirow{3}{*}{ Caso } & \multicolumn{3}{|c|}{ Lazo } & \multirow{2}{*}{$\mathbf{R}$} & \multirow{2}{*}{$\mathbf{L}$} & \multirow{2}{*}{$\mathbf{X}$} & \multirow{2}{*}{$\mathbf{Z}$} \\
\hline & Sec. & Ancho & Long & & & & \\
\hline & {$\left[\mathrm{mm}^{2}\right]$} & [m] & [m] & {$[\mathrm{m} \Omega]$} & {$[\mu \mathrm{Hy}]$} & {$[\mathrm{m} \Omega]$} & {$[\mathrm{m} \Omega]$} \\
\hline 1 & 35 & 1,0 & 2,85 & 5,8 & 10,1 & 3,2 & 6,6 \\
\hline 2 & 35 & 1,0 & 2,85 & 5,8 & 10,1 & 3,2 & 6,6 \\
\hline 3 & 95 & 1,0 & 4,45 & 2,75 & 9,3 & 2,92 & 4,01 \\
\hline 4 & 95 & 1,0 & 4,45 & 2,75 & 9,3 & 2,92 & 4,01 \\
\hline 5 & 95 & 1,5 & 4,00 & 2,75 & 9,02 & 2,83 & 3,95 \\
\hline 6 & 95 & 0,5 & 4,75 & 2,75 & 8,6 & 2,7 & 3,86 \\
\hline
\end{tabular}




\subsubsection{Resultados Obtenidos con cálculo y medición de campo magnético - Lazo único}

Una vez determinado el valor de la corriente inducida en el lazo en módulo y fase, se realizaron cálculos de valores de campo magnético y estos se compararon con mediciones.

Para las mediciones de campo magnético se utilizó un medidor de campo de tres ejes cuyo error de medición es del 8\%.

Los resultados se presentan por medio de perfiles transversales para los cálculos (C) y mediciones (M) de campo magnético en los casos experimentales presentados anteriormente.

En línea llena se representan los valores obtenidos a través de simulaciones. Los puntos representan los resultados obtenidos en mediciones. Para cada caso se utilizan los mismos colores en cálculos y mediciones.

Antes de energizar el circuito se midió el campo magnético de fondo presente, sin energizar la instalación.

En las Fig. 74 y Fig. 75 se presentan los resultados obtenidos para los casos "1 a 4". Las diferencias entre estas figuras comprenden dos aspectos, cambia la sección del conductor del lazo y el tamaño del mismo. Se obtienen mejores resultados en el caso de un mayor lazo y una mayor sección.

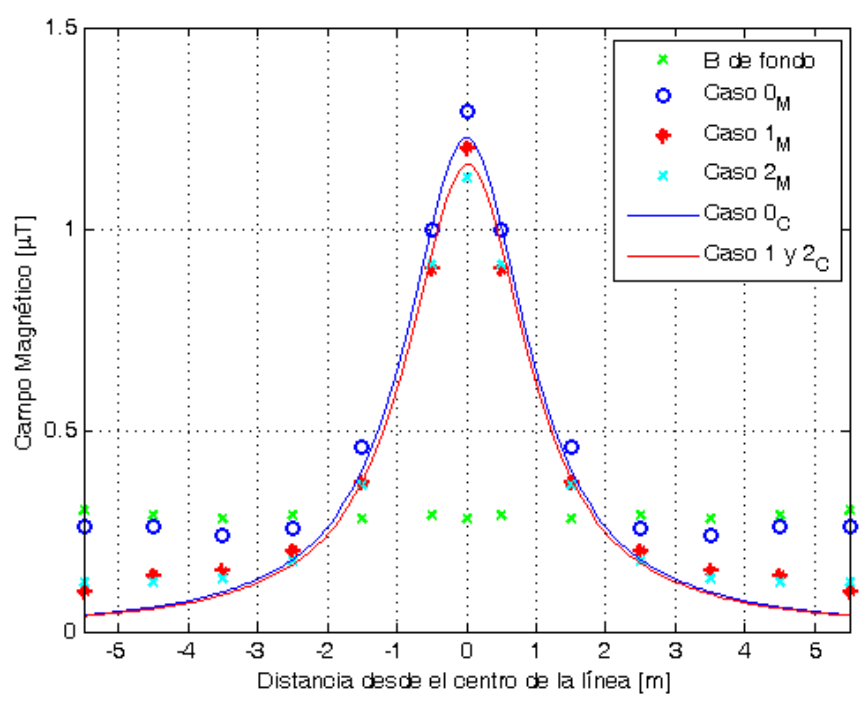

Fig. 74 - Perfiles obtenidos para el casos “1” y “2”, con I=14,9A, valores medidos y calculados. 


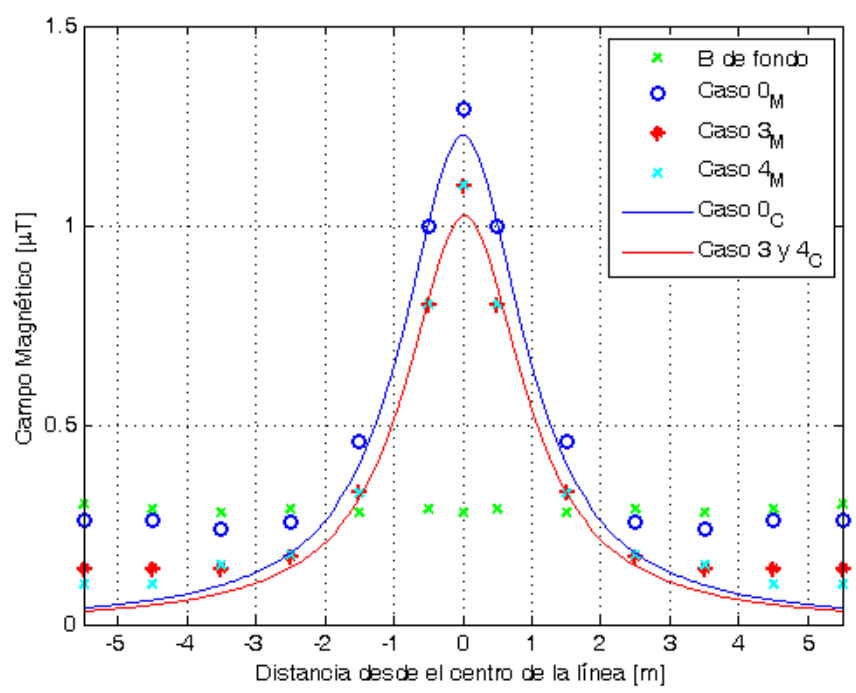

Fig. 75 - Perfiles obtenidos para los casos “3 y 4", con I=14,9A, valores medidos y calculados.

El la Fig. 76 se presentan los resultados obtenidos para una misma sección de conductor, $90 \mathrm{~mm}^{2}$, pero considerando distintas geometrías de lazo. Se presenta únicamente el perfil hacia uno de los lados.

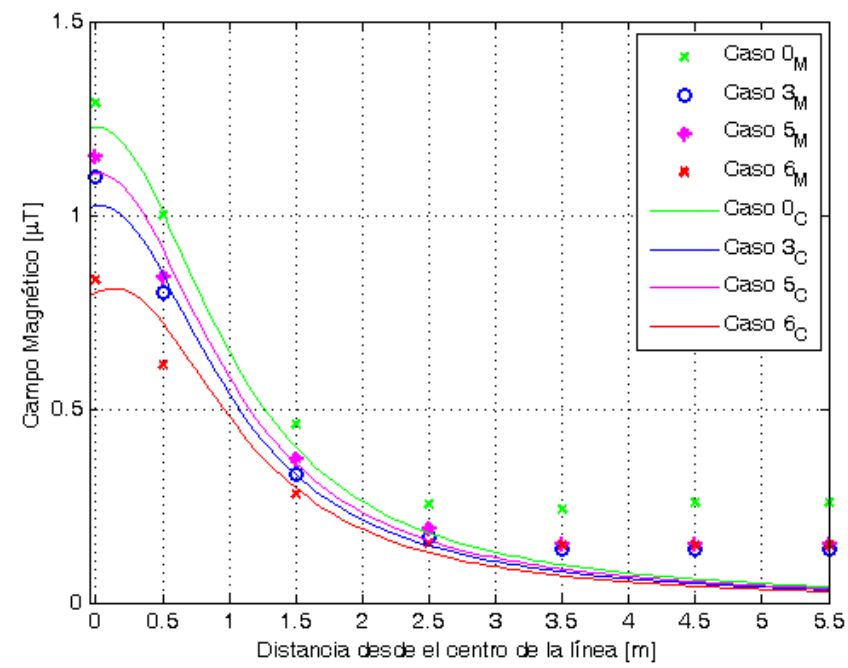

Fig. 76 - Perfiles obtenidos para los casos “0, 3, 5 y 6”, con I=14,9A, valores medidos y calculados. Sección del conductor $90 \mathrm{~mm}^{2}$.

\subsubsection{Análisis de Resultados}

Con respecto a las corrientes inducidas en los lazos se puede concluir que cuando se consideran conductores de mayor sección, la resistencia es menor y esto trae aparejado una disminución de la impedancia.

Si se modifica la geometría del lazo sin modificar la sección y la longitud del conductor, modificando el ancho y largo del lazo, se aprecia que la reactancia inductiva se modifica, y consecuentemente hay cambios en la impedancia. Pero esto no necesariamente se refleja en un aumento de la corriente inducida, ya que una configuración puede arrojar una mayor impedancia, pero las líneas de flujo concatenadas son mayores y la tensión inducida puede resultar mayor. 
Se debe notar que cuando se realizan mediciones, cabe la posibilidad de que existan fuentes externas de campo magnético, de frecuencia industrial, en las proximidades. Esto puede apreciarse en todos los casos presentados. Estas fuentes pueden además variar con el tiempo, por lo que es posible que en diferentes momentos se obtengan valores diferentes. Antes de implementar los circuitos descriptos se midió el nivel existente sin energizar la instalación y se encontró que algunos de estos valores pueden resultar influyentes en ciertas zonas.

Se verificaron los valores calculados a través de mediciones para las alternativas geométricas y se verificaron excitando el circuito para secuencias de fases diferentes.

En la Fig. 77 se aprecian la representación fasorial de los valores calculados y medidos para secuencia RST (Directa) y RTS (Inversa) en el caso en que se empleó un conductor de $35 \mathrm{~mm}^{2}$. Los valores de las corrientes por los lazos se multiplicaron por 10 para poder apreciar el módulo en los diagramas fasoriales.

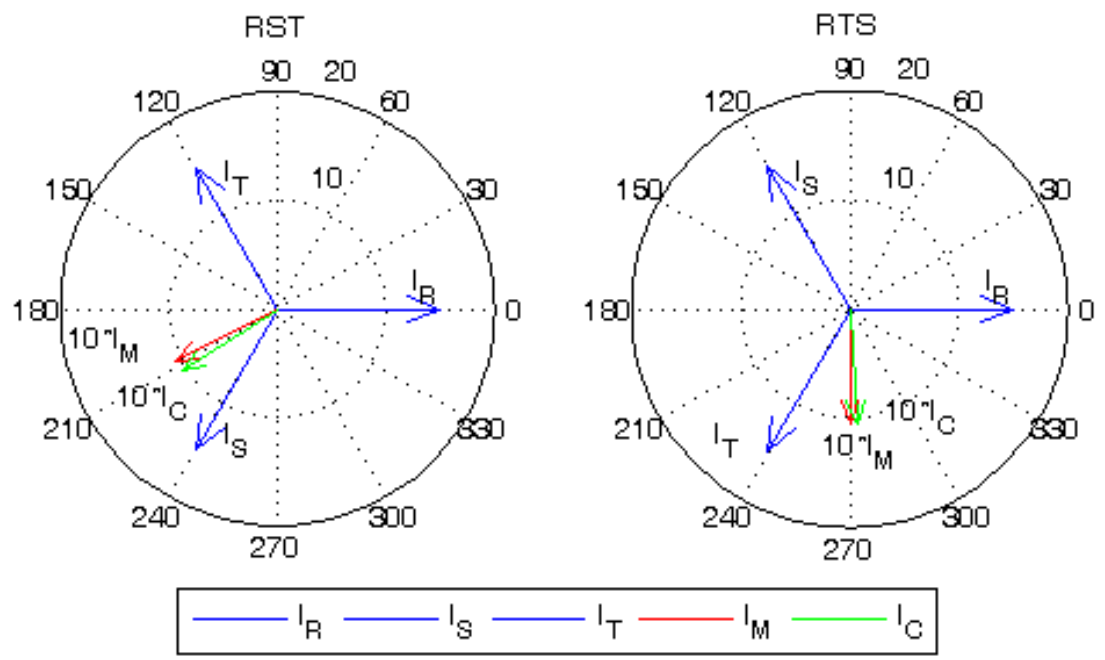

Fig. 77 - Corrientes de fase y corriente por lazo, calculada y medida para secuencia directa e inversa utilizando conductores de $35 \mathrm{~mm}^{2}$.

Respecto del campo magnético entre los valores medidos y calculados, estas diferencias caen dentro del error del instrumento de medición ya que en todos los casos están por debajo del $6 \%$.

Por otro lado a medida que las mediciones se apartan del centro del sistema, se aprecia que existen diferencias mayores a las indicadas previamente, esto se debe a que en dicha zona existe influencia de fuentes externas de campo que no se contemplan en los cálculos. Esto puede verificarse con las mediciones realizadas en vacío.

\subsubsection{Comentarios}

El objetivo general fue implementar la utilización de estos lazos y generar una herramienta de cálculo que permita conocer el comportamiento ante diferentes características geométricas de los lazos.

Se validó el modelo planteado a través de mediciones. Las diferencias encontradas en todos los casos estuvieron dentro de los errores de los instrumentos. Respecto de los módulos de las corrientes inducidas son prácticamente iguales, mientras que la diferencia en la fase cae dentro del error de los instrumentos utilizados.

Respecto del campo magnético, la herramienta de cálculo arroja resultados similares a las mediciones (dentro del error del instrumento utilizado), en la zona próxima al centro de la 
línea. A medida que se evalúa el campo magnético en puntos más alejados de esta zona se encuentran mayores diferencias, que se deben a fuentes externas de campo magnético, no contempladas en el modelo.

En los cálculos no se tuvo en cuenta la influencia de la conductividad de la tierra, todos los casos fueron implementados sobre la superficie de la tierra y no se observó la influencia de esta en los valores medidos con respeto a los calculados.

Se considera que la herramienta desarrollada cumple con el objetivo de brindar resultados aceptables a la hora de implementar lazos pasivos para mitigar el campo magnético y predecir el comportamiento de los mismos en determinadas zonas de interés.

\subsubsection{Resultados obtenidos con cálculos y mediciones- Más de un lazo}

Se implementó otra configuración para los conductores de fase, dispuestos a nivel del suelo, pero separados 0,5 m. Se dispusieron 1, 2, 3 y 4 lazos pasivos con distintas configuraciones. Se emplearon para los lazos conductores de 95 y $70 \mathrm{~mm}^{2}$.

Por medio de mediciones se determinó la resistencia de los lazos empleados, se midieron las resistencias de los conductores y de los empalmes realizados para cerrar el lazo.

\subsubsection{Configuraciones de lazo pasivo}

En la Fig. 78 se presenta la configuración utilizada en las mediciones, se aprecian los conductores de fase y un lazo pasivo.

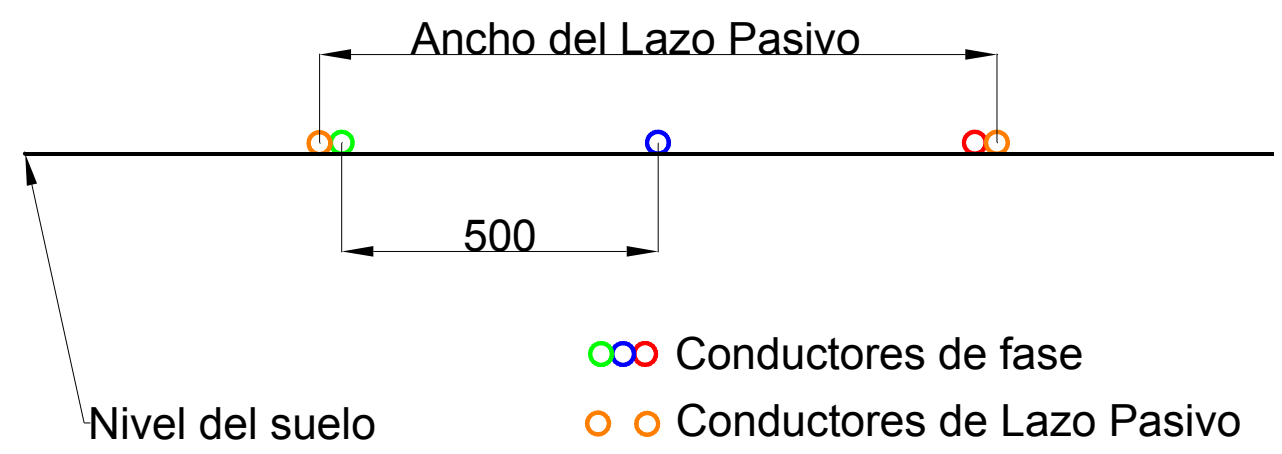

Fig. 78 - Configuración utilizada para mediciones en laboratorio.

En la Fig. 79 se presenta una imagen, en donde se pueden observar los conductores de fase, cuatro lazos pasivos y dos pinzas de corriente empleadas para las mediciones de corriente inducida. 


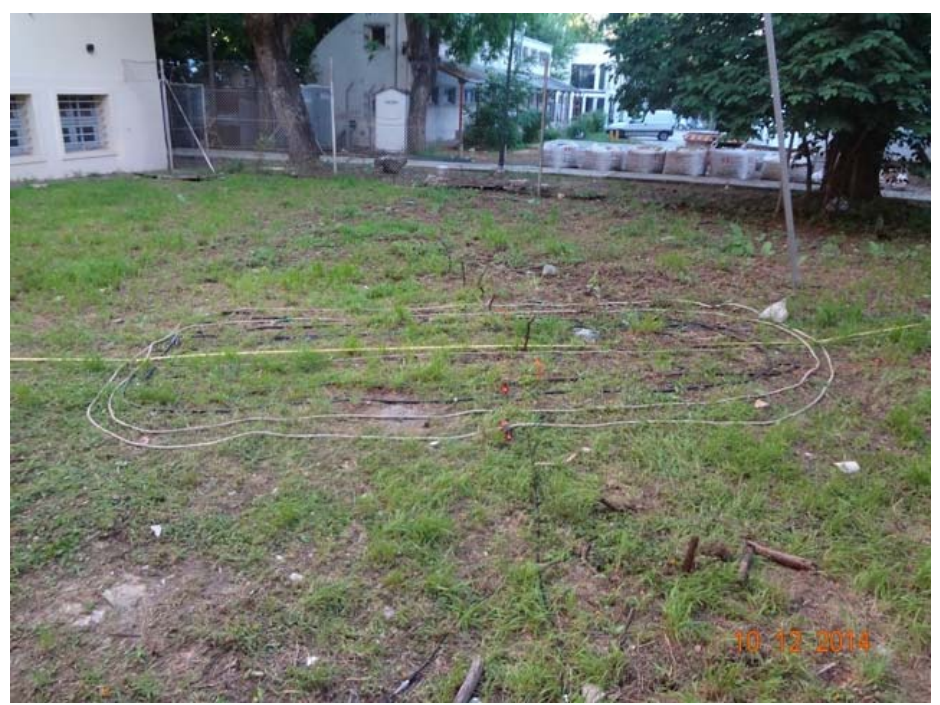

Fig. 79 - Arreglo montado para mediciones, con cuatro lazos Pasivos.

En la Tabla XII se presentan las características de los conductores y los lazos montados.

Tabla XII - Geometrías empleadas para las pruebas, en campo.

\begin{tabular}{|c|c|c|c|c|}
\hline \multirow{4}{*}{ Caso } & \multicolumn{2}{|c|}{ Conductor } & \multicolumn{2}{c|}{ Lazo } \\
\cline { 2 - 5 } & Sección & Radio & \multicolumn{2}{c|}{ Configuración } \\
\cline { 2 - 5 } & {$\left[\mathbf{m m}^{\mathbf{2}}\right]$} & {$[\mathbf{m m}]$} & Ancho & Largo \\
\hline 7 & 95 & 6,5 & 1 & 4,25 \\
\hline 8 & 95 & 6,5 & 1 & 4,35 \\
\hline \multirow{3}{*}{9} & 95 & 6,5 & 1 & 4,25 \\
\cline { 2 - 5 } & 95 & 6,5 & 1 & 4,35 \\
\hline \multirow{3}{*}{10} & 95 & 6,5 & 1 & 4,25 \\
\cline { 2 - 5 } & 95 & 6,5 & 1,4 & 4,2 \\
\hline \multirow{4}{*}{11} & 95 & 6,5 & 1 & 4,25 \\
\cline { 2 - 5 } & 95 & 6,5 & 1,4 & 4,35 \\
\cline { 2 - 5 } & 70 & 5,25 & 1 & 12,4 \\
\hline \multirow{4}{*}{13} & 95 & 6,5 & 1 & 4,25 \\
\cline { 2 - 5 } & 95 & 6,5 & 1,4 & 4,35 \\
\hline & 70 & 5,25 & 1,8 & 12,4 \\
\hline & 95 & 6,5 & 1 & 4,25 \\
\cline { 2 - 5 } & 95 & 6,5 & 1,4 & 4,35 \\
\hline & 70 & 5,25 & 1,8 & 12,4 \\
\hline
\end{tabular}

\subsubsection{Medición de corriente}

Para la medición de la corriente en módulo y fase se empleó un Analizador de Energía FLUKE 434, este es capaz de realizar mediciones de corriente en módulo y fase. Se utilizaron pinzas amperimétricas FLUKE i400S, empleadas en el rango de $40 \mathrm{~A}$. Se contaron con pinzas con rango de $5 \mathrm{~A}$, pero no fue posible el empleo de estas por dos motivos, en algunos casos el rango de corrientes a medir fue mayor que $5 \mathrm{~A}$ y en otros casos el diámetro exterior de los conductores empleados era mayor que el espacio disponible con la pinza cerrada.

En la Fig. 80 se puede observar el instrumento de medición y el control de la fuente de corriente utilizada para las pruebas. 
En la Tabla XIII se presentan los resultados obtenidos en las mediciones de corriente y los obtenidos por cálculos. En dicha tabla se observa que cuando se disponen los conductores de los lazos muy próximos a los conductores de fase, el error entre el cálculo y las mediciones de corriente aumenta. Esto se debe a los efectos de proximidad entre diferentes conductores, no considerados en el modelo de cálculo.

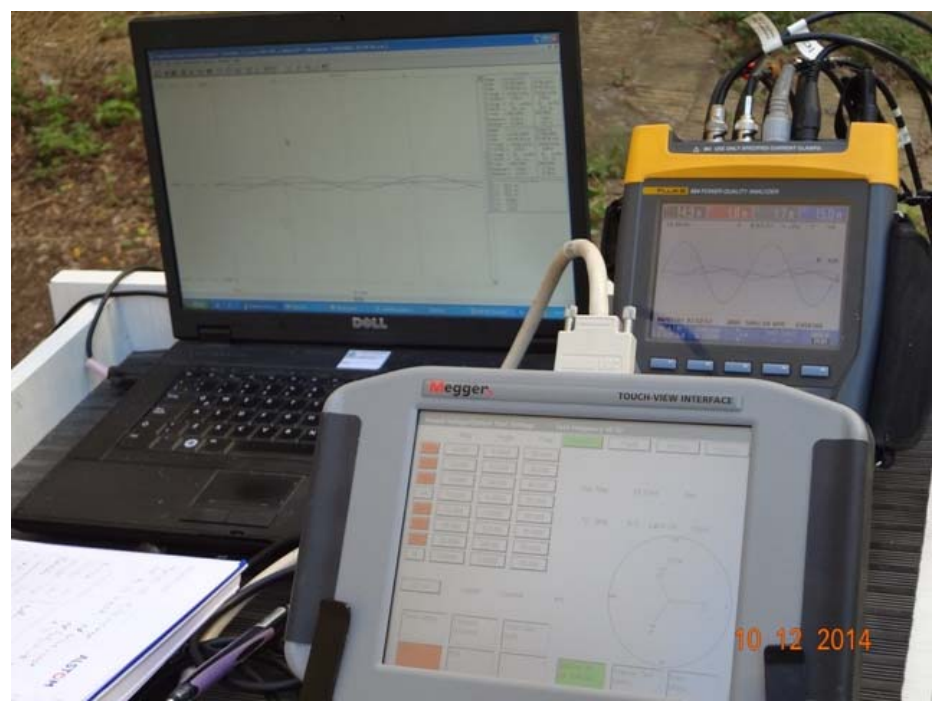

Fig. 80 - Elementos para medición y control de la corriente.

Tabla XIII - Corrientes medidas y calculadas.

\begin{tabular}{|c|c|c|c|c|c|c|}
\hline \multirow{3}{*}{ Caso } & \multicolumn{4}{|c|}{ Corriente } & \multirow{2}{*}{\multicolumn{2}{|c|}{ Error }} \\
\hline & \multicolumn{2}{|c|}{ Medida } & \multicolumn{2}{|c|}{ Calculada } & & \\
\hline & [A] & Fase $\left[{ }^{\circ}\right]$ & [A] & Fase $\left[{ }^{\circ}\right]$ & [\%] & Fase $\left[{ }^{\circ}\right]$ \\
\hline 7 & 6,3 & 7,0 & 7,4 & 8 & 15 & 1 \\
\hline 8 & 6 & 6,0 & 7,2 & 8 & 17 & 2 \\
\hline \multirow{2}{*}{9} & 4,73 & $-1,4$ & 4,28 & -4 & -11 & 3 \\
\hline & 3,8 & $-8,0$ & 4,25 & -8 & 11 & 0 \\
\hline \multirow{2}{*}{10} & 5,57 & 13,0 & 6,7 & 14 & 17 & 1 \\
\hline & 2 & $-17,1$ & 1,8 & -18 & -11 & 1 \\
\hline \multirow{3}{*}{11} & 4,4 & $-3,6$ & 4,1 & -4 & -7 & 0 \\
\hline & 1,6 & $-39,0$ & 1,4 & -22 & -14 & -17 \\
\hline & 3,1 & $-3,0$ & 3,5 & 0 & 11 & -3 \\
\hline \multirow{3}{*}{12} & 5,5 & 6,0 & 6,6 & 14 & 17 & 8 \\
\hline & 1,7 & $-26,0$ & 1,62 & $-25,8$ & -5 & 0 \\
\hline & 0,9 & $-26,0$ & 1 & -21 & 10 & -5 \\
\hline \multirow{4}{*}{13} & 5,5 & 6,0 & 6,5 & 14 & 15 & 8 \\
\hline & 1,7 & $-27,0$ & 1,55 & -27 & -10 & 0 \\
\hline & 0,8 & $-31,0$ & 0,9 & -26 & 11 & -5 \\
\hline & 0,5 & $-32,0$ & 0,58 & -26 & 14 & -6 \\
\hline
\end{tabular}




\subsubsection{Medición de campo magnético.}

Para la medición de campo magnético se utilizó un medidor de tres ejes con una zonda de $100 \mathrm{~cm}^{2}$.

En las figuras en color Azul se indican los valores de campo obtenidos sin energizar los conductores de fase.

En color Rojo se indican los valores de campo sin introducción de los lazos pasivos.

En color Verde se presentan los valores de campo obtenidos con la introducción de lazos pasivos. Con trazo continuo se indican los valores obtenidos considerando las corrientes calculadas (IC) y en trazo discontinuo se indican los valores de campo obtenidos considerando las corrientes medidas. La corriente medida en los conductores de fase fue de 14,7 A en todos los casos.

En la Fig. 81 se presentan los resultados obtenidos para el caso 7. En la Fig. 82 se presentan los resultados correspondientes al caso 8. Estos dos casos corresponden a la implementación de un lazo pasivo, considerando un conductor de $95 \mathrm{~mm}^{2}$.

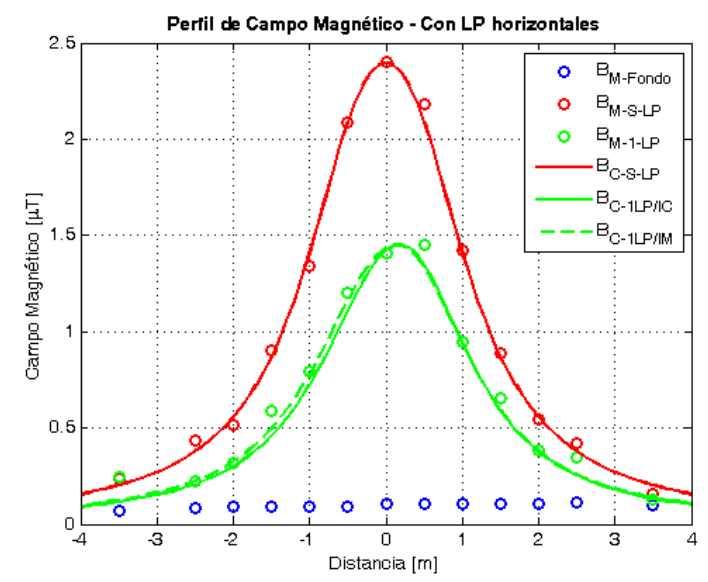

Fig. 81 - Perfiles obtenidos para el caso "7" (Verde), valores medidos y calculados. Se incluyen los perfiles sin energizar la instalación (Azul) y sin LP (Rojo).

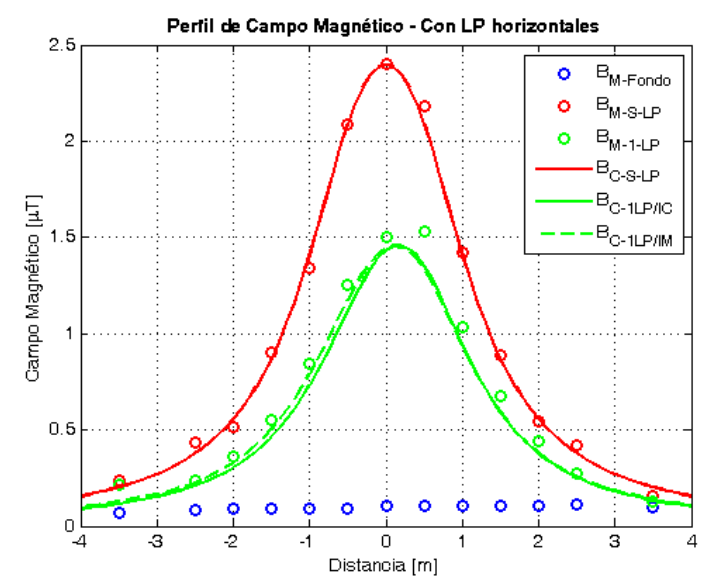

Fig. 82 - Perfiles obtenidos para el caso "8" (Verde), valores medidos y calculados. Se incluyen los perfiles sin energizar la instalación (Azul) y sin LP (Rojo).

En la Fig. 83 se presentan los resultados obtenidos para el caso 9. En la Fig. 84 se presentan los resultados correspondientes al caso 10 . Estos dos casos corresponden a la implementación de dos lazos pasivos, considerando conductores de $95 \mathrm{~mm}^{2}$. 


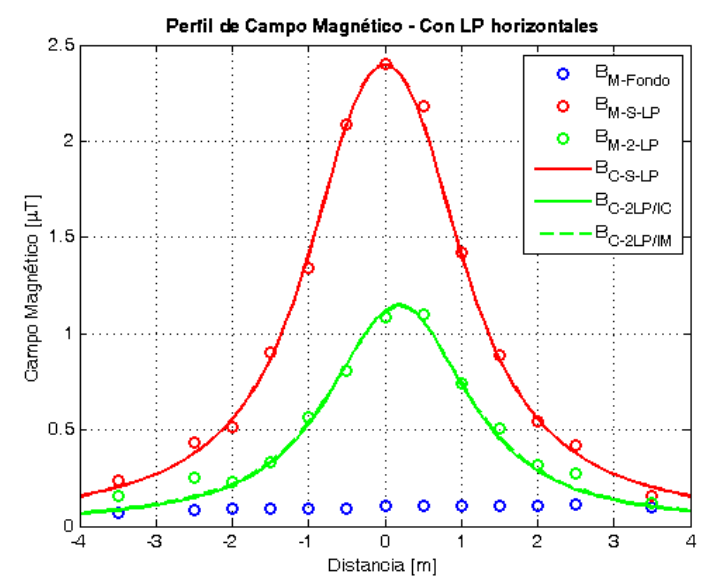

Fig. 83 - Perfiles obtenidos para el caso "9" (Verde), valores medidos y calculados. Se incluyen los perfiles sin energizar la instalación (Azul) y sin LP (Rojo).

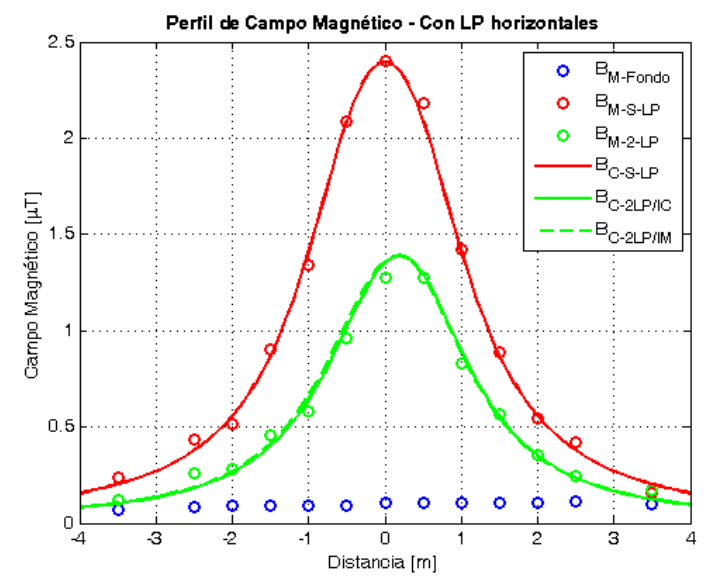

Fig. 84 - Perfiles obtenidos para el caso "10" (Verde), valores medidos y calculados. Se incluyen los perfiles sin energizar la instalación (Azul) y sin LP (Rojo).

En la Fig. 85 se presentan los resultados obtenidos para el caso 11. En la Fig. 86 se presentan los resultados correspondientes al caso 12. Estos dos casos corresponden a la implementación de tres lazos pasivos, considerando dos conductores de $95 \mathrm{~mm}^{2}$ y un conductor de $70 \mathrm{~mm}^{2}$.

En la Fig. 87 se presentan los resultados obtenidos para el caso 13. Este caso corresponde a la implementación de cuatro lazos pasivos, considerando dos conductores de $95 \mathrm{~mm}^{2}$ y dos conductores de $70 \mathrm{~mm}^{2}$. 


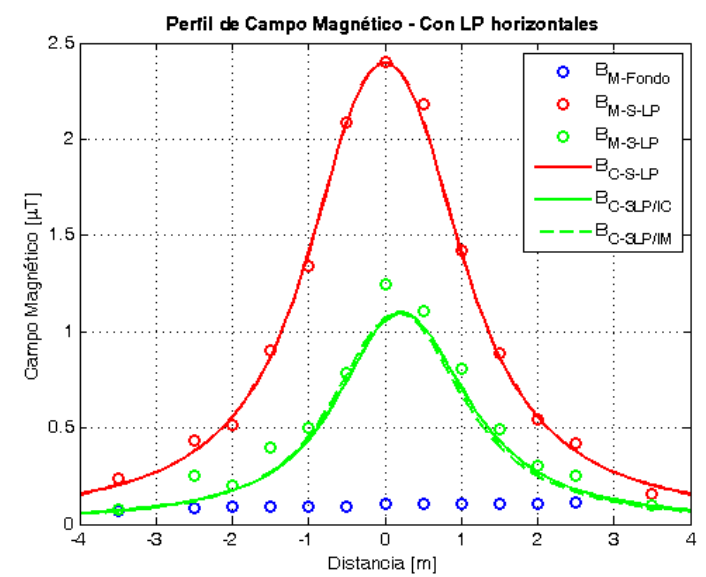

Fig. 85 - Perfiles obtenidos para el caso "11" (Verde), valores medidos y calculados. Se incluyen los perfiles sin energizar la instalación (Azul) y sin LP (Rojo).

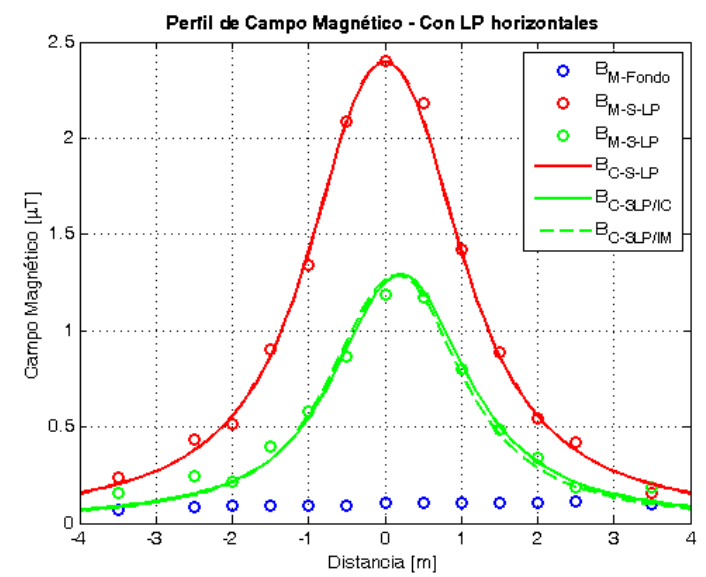

Fig. 86 - Perfiles obtenidos para el caso "12" (Verde), valores medidos y calculados. Se incluyen los perfiles sin energizar la instalación (Azul) y sin LP (Rojo).

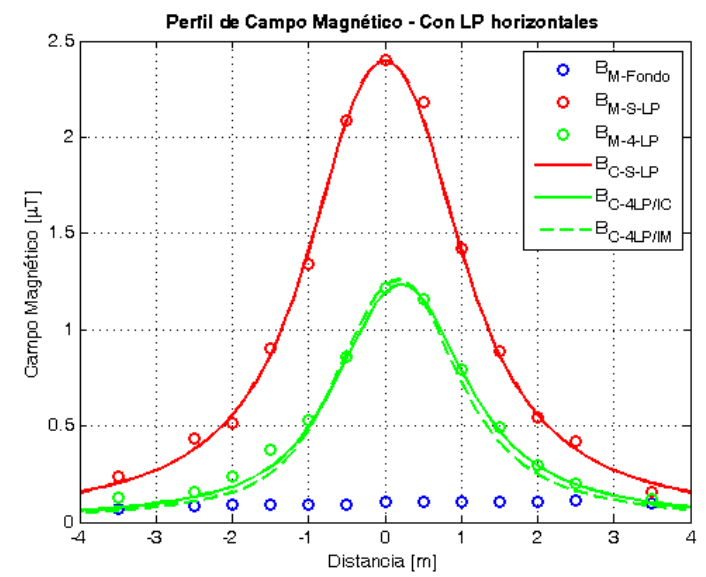

Fig. 87 - Perfiles obtenidos para el caso "13" (Verde), valores medidos y calculados. Se incluyen los perfiles sin energizar la instalación (Azul) y sin LP (Rojo). 


\subsection{Aplicación de lazos pasivos}

En este punto se busca analizar el efecto de los lazos considerando diferentes posiciones, características y cantidad. En la Fig. 88 se presenta una vista en donde se aprecian los conductores de fases y lazos pasivos (Naranja) sobre los mismos.

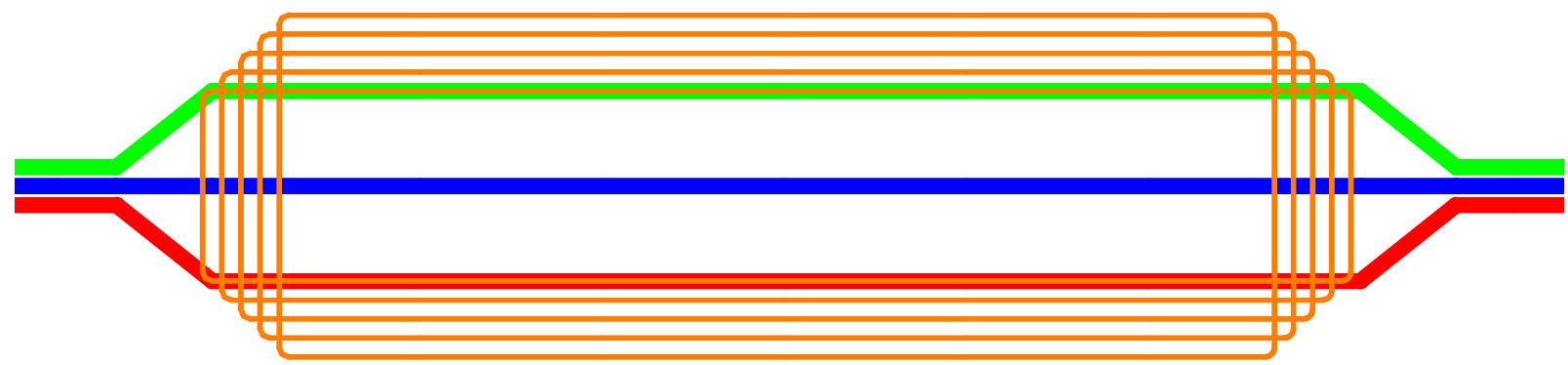

Fig. 88 - Ubicación de lazos pasivos en la zona de empalmes, vista superior.

En la Fig. 89 se pueden apreciar imágenes tomadas durante el proceso de instalación de lazos pasivos en una fosa de empalmes, correspondiente a la traza de cables subterráneos de Alta Tensión. En a) se aprecia la instalación en uno de los extremos, en b) la zona central del mismo y en c) una vista general de la instalación.

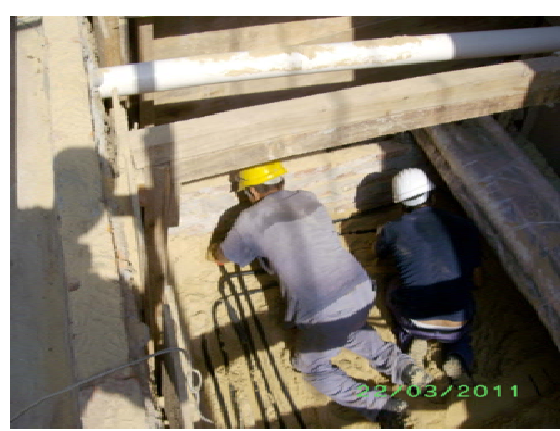

a) Extremo

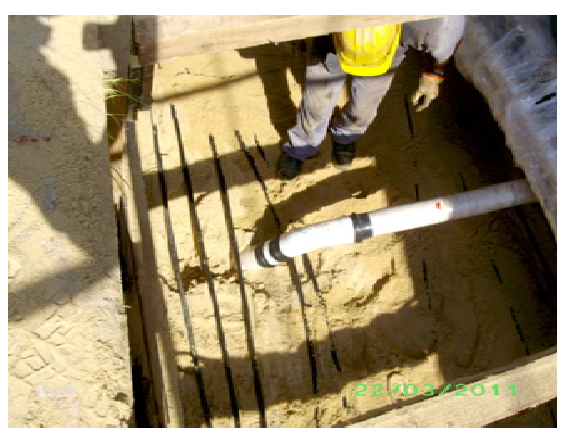

b) Zona Central

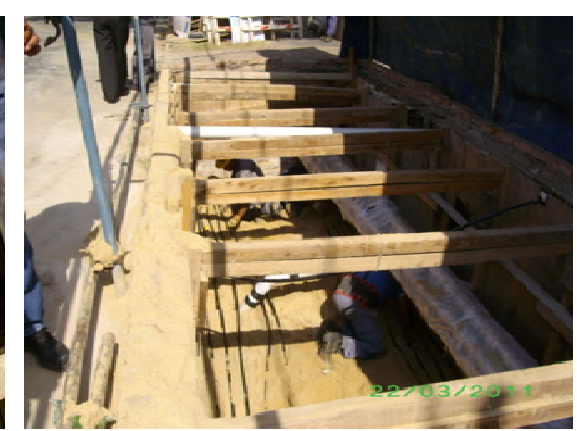

c) Vista general

Fig. 89 - Caso real, implementación

\subsection{Ubicación}

La ubicación de estos lazos depende de la zona de interés o zona en donde se pretende mitigar el campo magnético, en cables subterráneos la utilización de esta solución presenta un gran potencial en la zona de empalmes, las restricciones en lo que respecta a dimensiones serían las dimensiones de la fosa de empalmes (largo, ancho y profundidad) [ 41 ] [ 42 ] [ 43 ].

Otro factor de importancia es la distancia que deben guardar los lazos con respecto a los conductores de fase, ya que debe prestarse especial atención en no ocasionar una limitación en la corriente máxima por los conductores de fase debido al calentamiento adicional que puedan provocar las corrientes inducidas por los conductores de los lazos.

En términos generales pueden disponerse los lazos en un mismo plano, por encima, por debajo o en el mismo plano de los conductores de fase. También se pueden disponer en el perímetro de los conductores de fase en distintos planos.

En la Fig. 90 se presenta la configuración en la que se disponen los lazos en planos por encima de los conductores de fase, es posible variar en número de lazos y la distancia entre 
los conductores de fase y el plano que contiene a los lazos. En la misma figura se representa esta situación, en donde se contempla la instalación de 1 a 9 lazos pasivos.

Esta situación puede considerarse también ubicando el plano de los lazos pasivos por debajo de los conductores de fase.
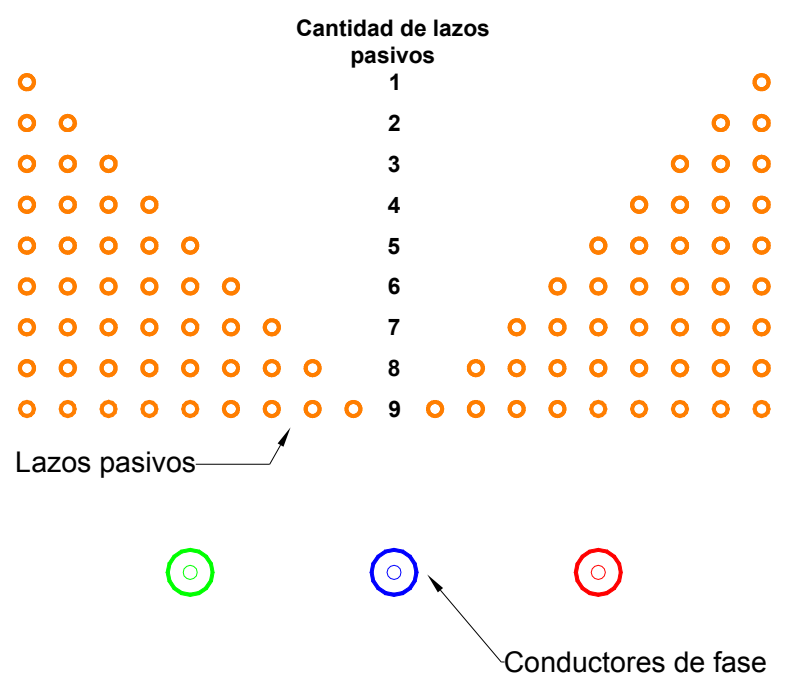

Fig. 90 - Disposición de lazos pasivos en planos por encima de los conductores de fase.

En la Fig. 91 se presenta un esquema con la disposición de lazos pasivos en planos por encima y por debajo de los conductores de fase.
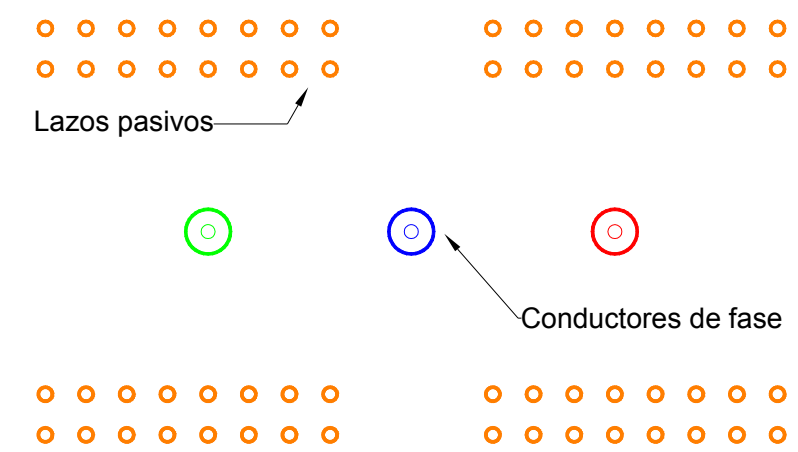

Fig. 91 - Disposición de lazos pasivos en planos por encima y debajo de los conductores de fase.

En la Fig. 92 se presenta una imagen en la que se disponen lazos pasivos en planos verticales a ambos lados de los conductores de fase.

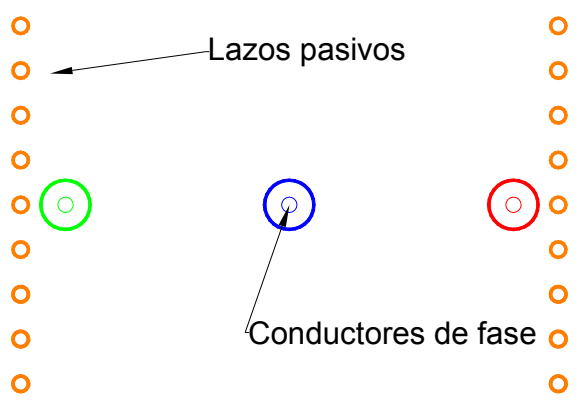

Fig. 92 - Disposición de lazos pasivos en planos laterales a los conductores de fase. 
En la Fig. 93 se observa la ubicación de lazos pasivos en el perímetro de los conductores de fase. Esta alternativa representa una combinación de las presentadas anteriormente.

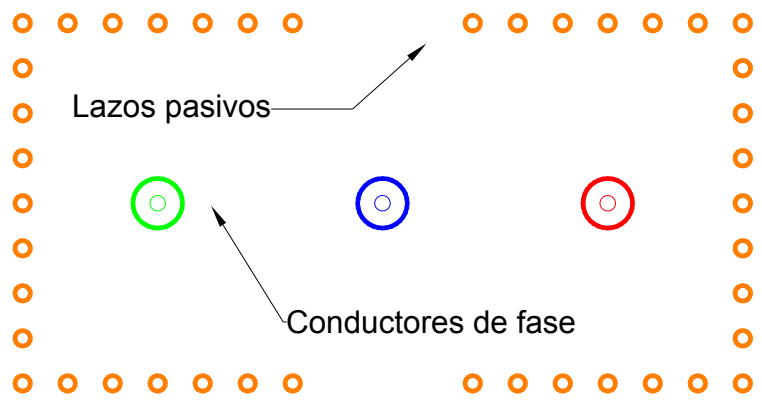

Fig. 93 - Disposición de lazos pasivos en el perímetro de los conductores de fase.

Las alternativas planteadas hasta aquí serán analizadas, evaluando el comportamiento desde el punto de vista de la mitigación del campo magnético.

\subsection{Factores de influencia}

En este punto se busca analizar distintos aspectos que influyen en el diseño de la configuración de los lazos pasivos.

Un factor importante es la proximidad entre los lazos pasivos y los conductores de fase, a medida que los lazos estén más próximos, mayor será la corriente inducida en ellos. Esto debe manejarse con cierta precaución, debido a que el diseño debe realizarse de forma tal, de no ocasionar una reducción en la capacidad de transportar corriente por los conductores de fase, en la jerga este fenómeno se conoce como "derating".

Se evalúan diferentes factores, como ser:

- Número de lazos empleado.

- Sección de conductores.

- Distancia entre lazos y conductores de fase.

- Configuración:

○ Lazos en un plano entre conductores de fase y zona de interés.

- Lazos dispuestos a un lado.

- Lazos en el perímetro

- Lazos dispuestos en diferentes planos.

A través de ejemplos se evalúan los puntos mencionados anteriormente.

El ejemplo que se plantea es el que se presentó en la Fig. 4, pero se considerará una corriente de $1000 \mathrm{~A}$, por los conductores de fase.

Se consideran diferentes secciones de cables de Baja Tensión disponibles en catálogos de fabricantes de cables, en este caso se consideró la Guía Técnica de Prysmian - Selección y Dimensionamiento de Conductores de Baja Tensión [ 44 ], de la misma se extrajeron los datos necesarios que se presentan en la Tabla XIV.

Tabla XIV - Características de conductores de BT considerados [ 44 ].

\begin{tabular}{|c|c|c|}
\hline $\begin{array}{c}\text { Sección de conductor } \\
{\left[\mathbf{m m}^{\mathbf{2}}\right]}\end{array}$ & $\begin{array}{c}\text { Radio } \\
{[\mathbf{m m}]}\end{array}$ & $\begin{array}{c}\text { Resistencia } \\
{[\mathbf{\Omega} / \mathbf{k m}]}\end{array}$ \\
\hline 185 & 10 & 0,129 \\
\hline
\end{tabular}




\begin{tabular}{|c|c|c|}
\hline $\begin{array}{c}\text { Sección de conductor } \\
{\left[\mathbf{m m}^{\mathbf{2}} \mathbf{]}\right.}\end{array}$ & $\begin{array}{c}\text { Radio } \\
{[\mathbf{m m}]}\end{array}$ & $\begin{array}{c}\text { Resistencia } \\
{[\mathbf{\Omega} / \mathbf{k m}]}\end{array}$ \\
\hline 240 & 12 & 0,0987 \\
\hline 300 & 10,35 & 0,0754 \\
\hline 400 & 11,5 & 0,0606 \\
\hline 500 & 13,2 & 0,0493 \\
\hline 630 & 15 & 0,0407 \\
\hline
\end{tabular}

Tabla XV - Configuraciones de lazos pasivos consideradas - Cables subterráneos.

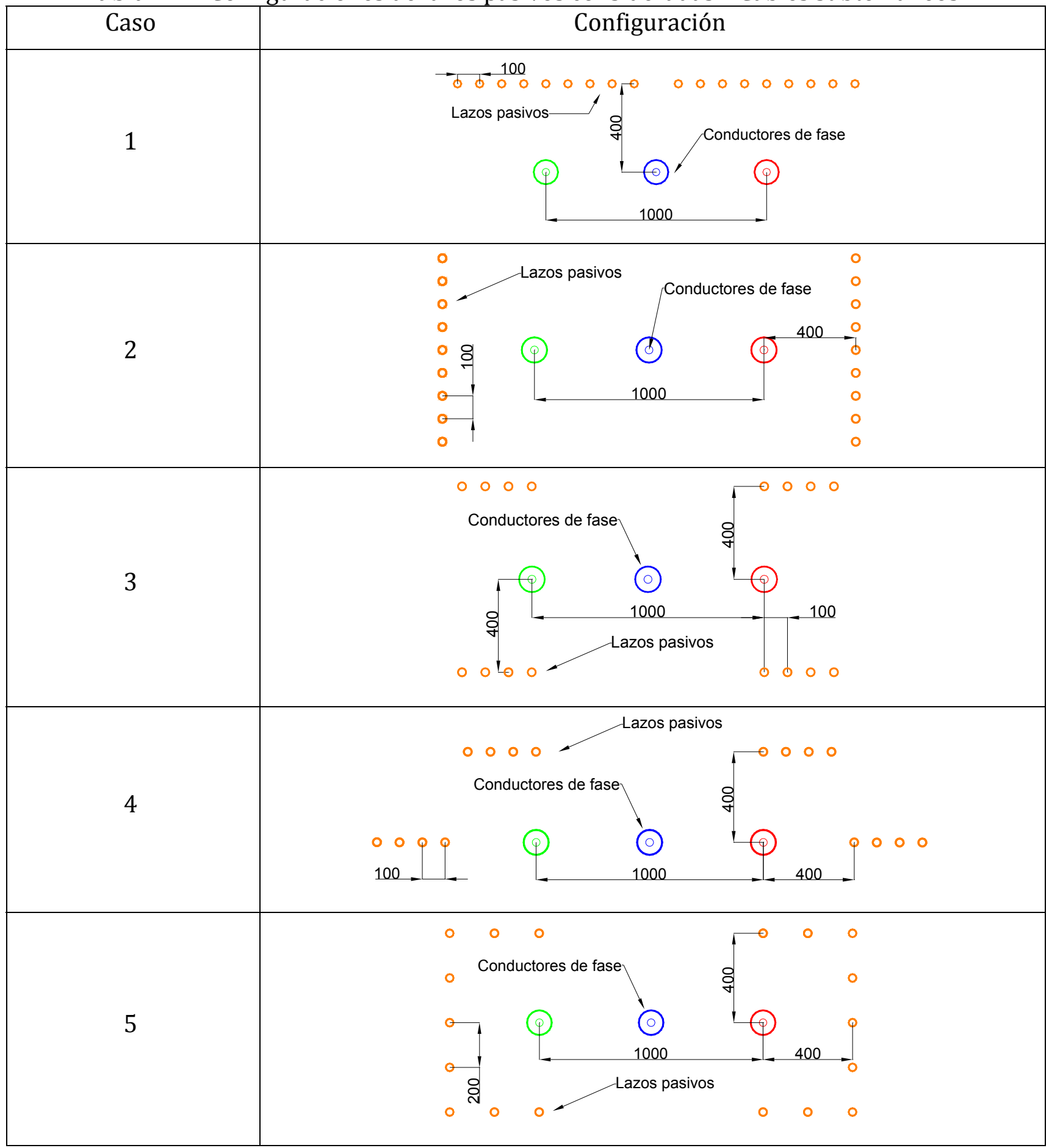




\subsubsection{Número de lazos pasivos empleados}

En primer lugar se disponen los lazos en un plano ubicado entre los conductores y la zona de interés, de forma similar a la presentada en la Fig. 90. Se disponen en primera instancia un lazo pasivo y se van adicionando lazos hasta ubicar 9.

La distancia entre los conductores de fase y el plano que contiene a los lazos pasivos es de $400 \mathrm{~mm}$. La sección de los conductores de los lazos para este caso es de $185 \mathrm{~mm}^{2}$. En la Fig. 94 se presenta un detalle de la instalación propuesta, Caso 1 - Tabla XV.

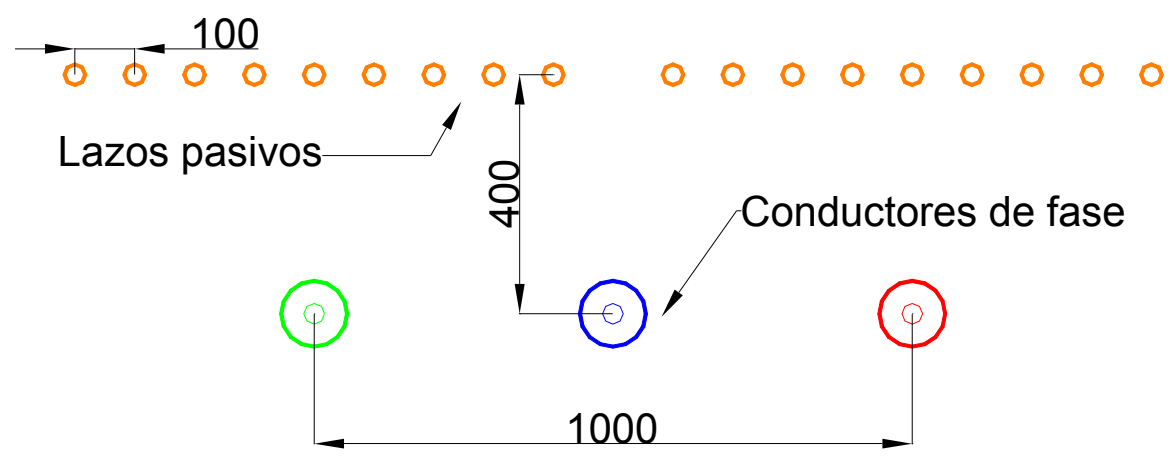

Fig. 94 - Ejemplo de aplicación, lazos pasivos ubicados en un plano paralelo al de los conductores de fase, entre estos y la zona de interés. Caso 1 - Tabla XV.

Para el ejemplo de la Fig. 94, se trazan los perfiles de campo magnético, a $1 \mathrm{~m}$ sobre el nivel del suelo, estos se presentan en la Fig. 95. En color rojo se aprecia el perfil obtenido sin lazos pasivos. La corriente considerada fue de $1000 \mathrm{~A}$. El valor máximo de campo fue de $20 \mu \mathrm{T}$, en el centro del empalme $(\mathrm{x}=0)$. En diferentes colores se presentan los perfiles obtenidos para diferentes números de lazos pasivos.

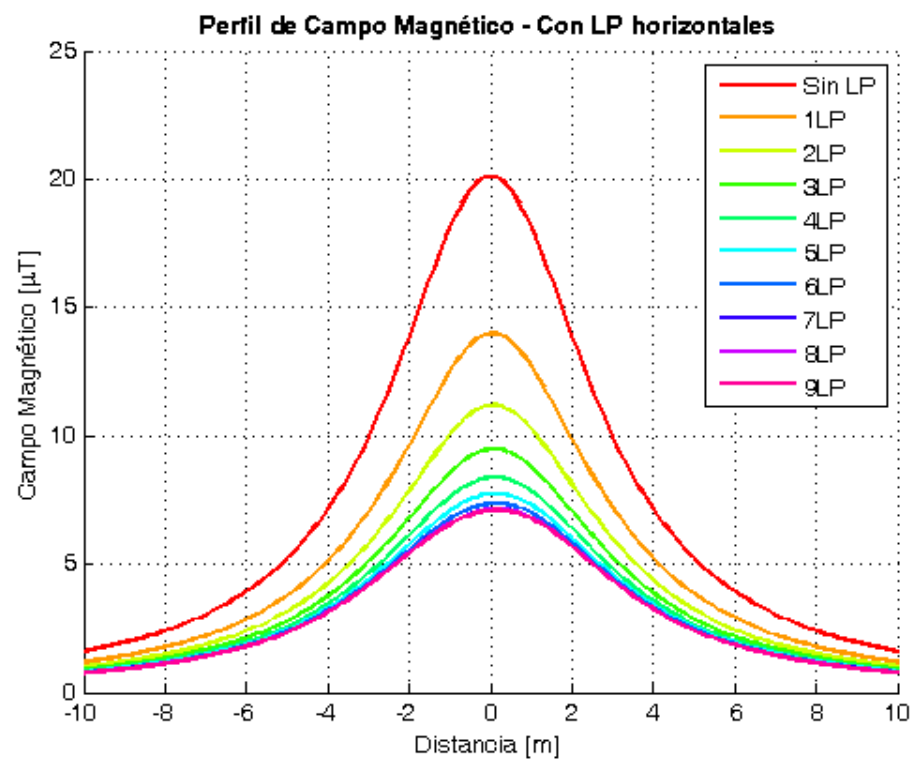

Fig. 95 - Perfiles de campo magnético con lazos pasivos en un plano horizontal entre los conductores de fase y la área de interés, I= $1000 \mathrm{~A}$, lazos de $185 \mathrm{~mm}^{2}$, Caso 1 - Tabla XV.

A medida que se incrementa el número de lazos se obtienen menores valores de campo magnético. En la Fig. 96 se trazan los perfiles de FA. Se observa que a medida que se incrementa el número de LP se produce un incremento en los valores de los perfiles del FA. 


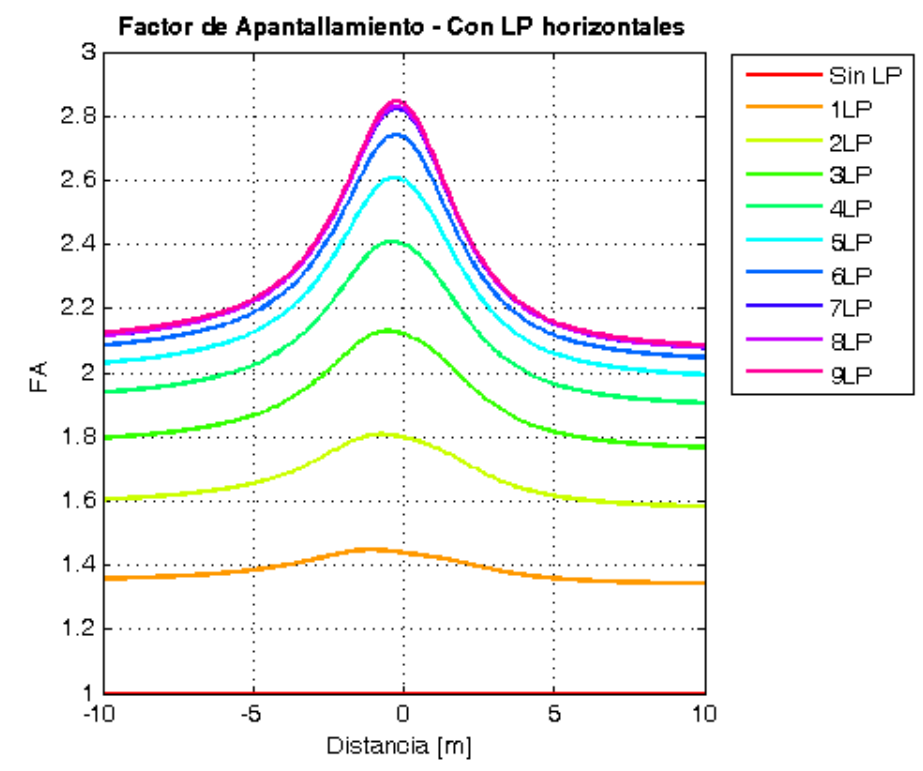

Fig. 96 - Perfiles de FA con lazos pasivos en un plano horizontal entre los conductores de fase y la área de interés, I= $1000 \mathrm{~A}$, lazos de 185 mm², Caso 1 - Tabla XV.

A partir del séptimo lazo, ya no se observan grandes diferencias en los perfiles de FA.

En la Fig. 97 se presenta otro ejemplo, en donde los lazos se disponen en diferentes planos a ambos lados de los conductores de fase, el primer lazo se dispone en el mismo plano que los conductores de fase y se van adicionando hacia la parte superior e inferior progresivamente, Caso 2 - Tabla XV.

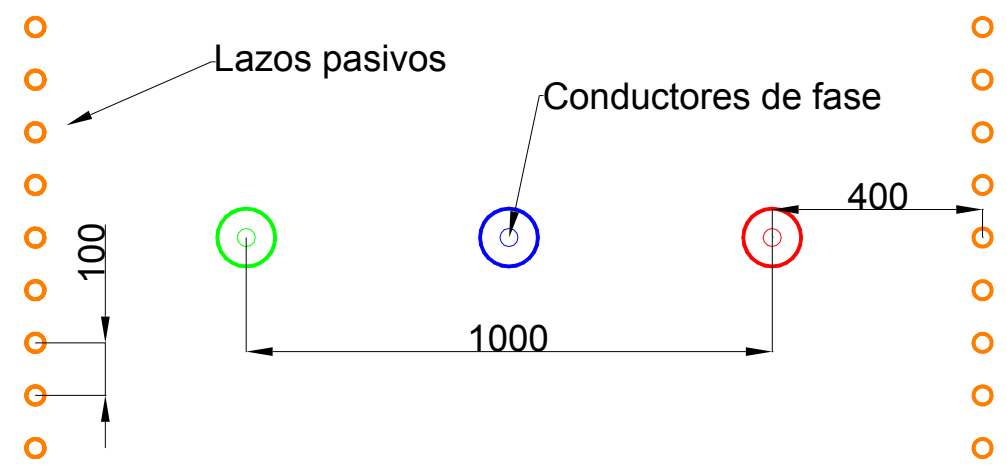

Fig. 97 - Ejemplo de aplicación, lazos pasivos ubicados en diferentes planos, todos los lazos tienen el mismo largo y ancho, Caso 2 - Tabla XV.

Para las condiciones planteadas en la Fig. 97, se trazan los perfiles de campo magnético a 1m sobre el nivel del suelo, estos se presentan en la Fig. 98, en color rojo se aprecia el perfil obtenido sin lazos pasivos. La corriente considerada fue de $1000 \mathrm{~A}$., el valor máximo de campo es de $20 \mu \mathrm{T}$, el centro del empalme $(\mathrm{x}=0)$. 


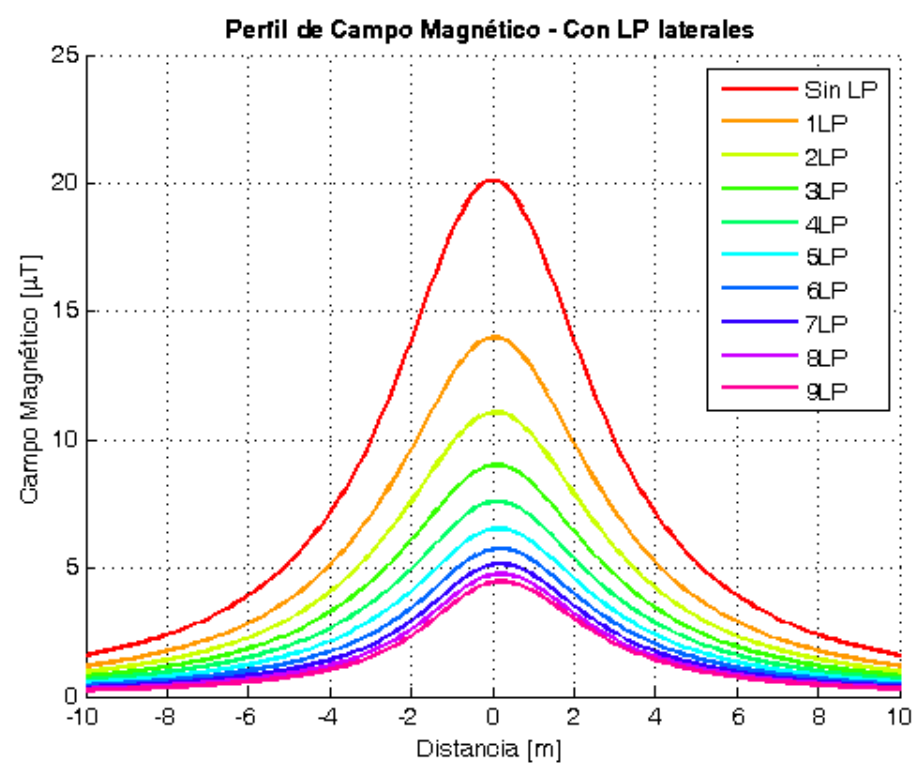

Fig. 98 - Perfiles de campo magnético con lazos pasivos en diferentes planos a ambos lados de los conductores de fase, I= $1000 \mathrm{~A}$, lazos de $185 \mathrm{~mm}^{2}$, Caso 2 - Tabla XV.

A medida que se introducen lazos se obtienen menores valores de campo magnético. En la Fig. 99 se trazan los perfiles de FA. Se observa que a medida que se incrementa el número de LP se produce un incremento en los valores de los perfiles del FA.

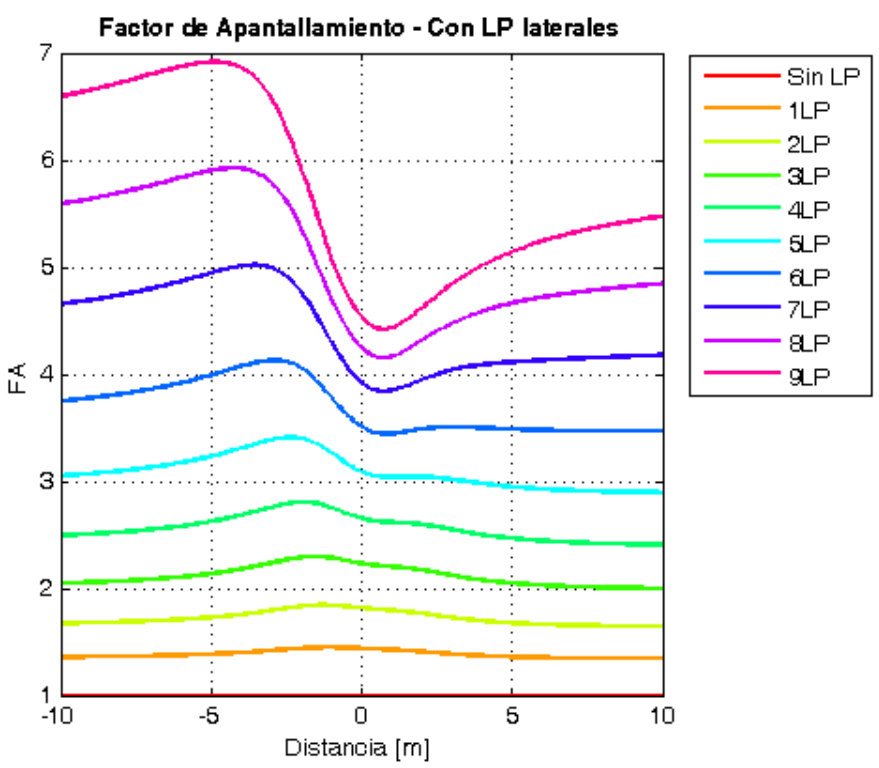

Fig. 99 - Perfiles de FA con lazos pasivos en diferentes planos a ambos lados de los conductores de fase, I= $1000 \mathrm{~A}$, lazos de $185 \mathrm{~mm}^{2}$, Caso 2 - Tabla XV.

Los perfiles para el FA son cada vez mayores, obteniéndose en el caso de 9 LP valores cercanos a 7, pero en la zona de mayor valor de campo magnético el correspondiente valor de FA es de 4,5.

Si se comparan estos dos ejemplos, se puede decir que el incremento en el número de LP provoca una reducción de los valores de campo magnético cada vez mayor.

Dependiendo de cuál sea la configuración elegida se pueden lograr mejores resultados. Para el caso de conductores en un mismo plano (Caso 1 - Tabla XV) se puede decir que con más de 
7 LP ya no se logran grandes cambios, pero en este caso el ancho y el largo de los lazos pasivos se va modificando.

En el segundo ejemplo (Caso 2 - Tabla XV) la geometría de los lazos no se modifica, pero si los planos en donde se van disponiendo estos.

\subsubsection{Sección del conductor}

En este punto se analiza lo que ocurre cuando se disponen conductores de diferentes secciones para una misma configuración. Para esto se consideran los ejemplos presentados en las Fig. 94 y Fig. 97, Caso 1 y 2 de la Tabla XV respectivamente, pero con diferentes secciones de conductores, 240, 300, 400, 500 y $630 \mathrm{~mm}^{2}$. Los perfiles obtenidos para conductores de $185 \mathrm{~mm}^{2}$ se presentaron en el inciso anterior.

En el Anexo IX, se presentan los perfiles de campo magnético obtenidos y los correspondientes al FA. Con estos resultados es posible realizar una comparación, el punto de mayor interés es el de mayor valor de campo magnético, $\mathrm{x}=0$, por lo que se analizan los resultados obtenidos en este punto. Se trazan gráficos con los valores de campo magnético en $\mathrm{x}=0$, para diferentes números de lazos y secciones consideradas, a su vez se trazan los valores obtenidos para el FA en dicho punto. En las Fig. 100 y Fig. 101 se presentan los valores de campo magnético.

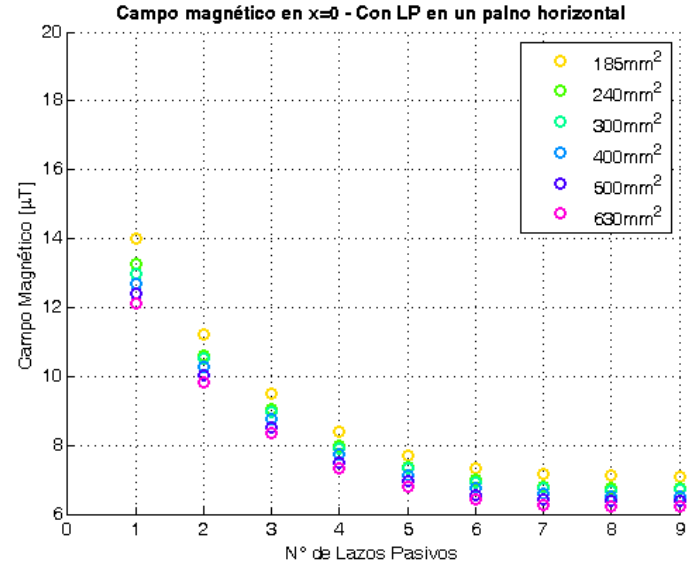

Fig. 100 - Valores de campo magnético en $\mathrm{x}=0 \mathrm{a}$, considerando lazos pasivos en un plano, Caso 1 - Tabla XV.

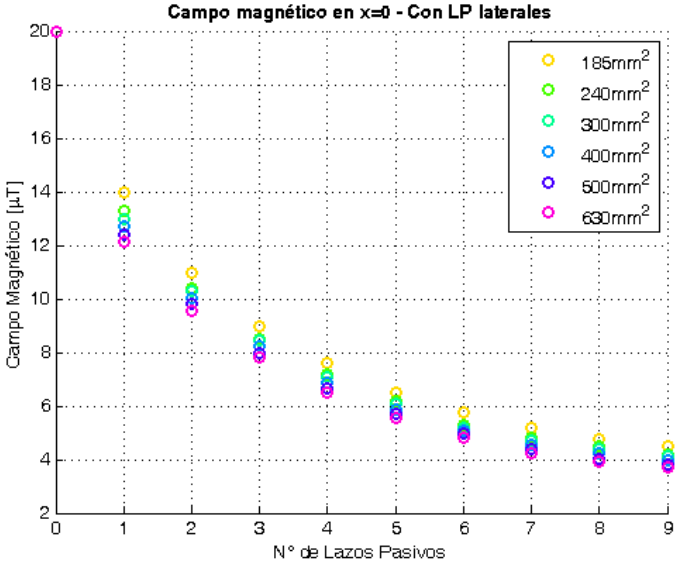

Fig. 101 - Valores de campo magnético en $\mathrm{x}=0$, considerando lazos pasivos a ambos lados de los conductores de fase, Caso 2 Tabla XV.

En las Fig. 102 y Fig. 103, se presentan los valores correspondientes al FA. 


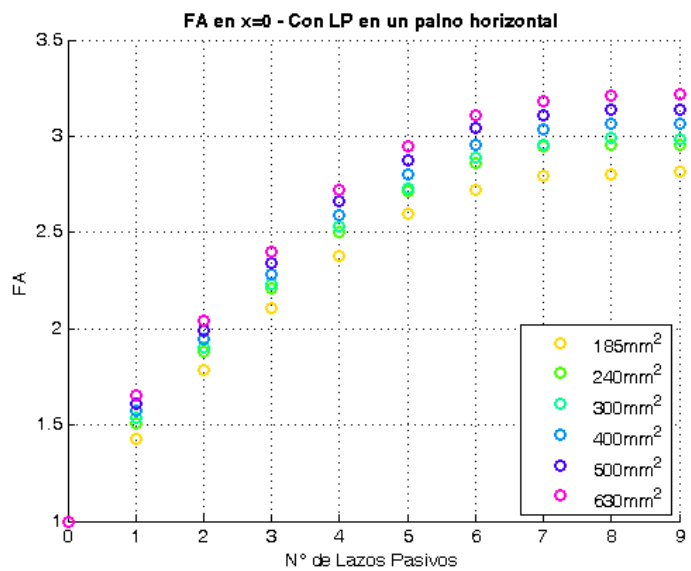

Fig. 102 - Valores de FA en $\mathrm{x}=0 \mathrm{a}$, considerando lazos pasivos en un plano, Caso 1 - Tabla XV.

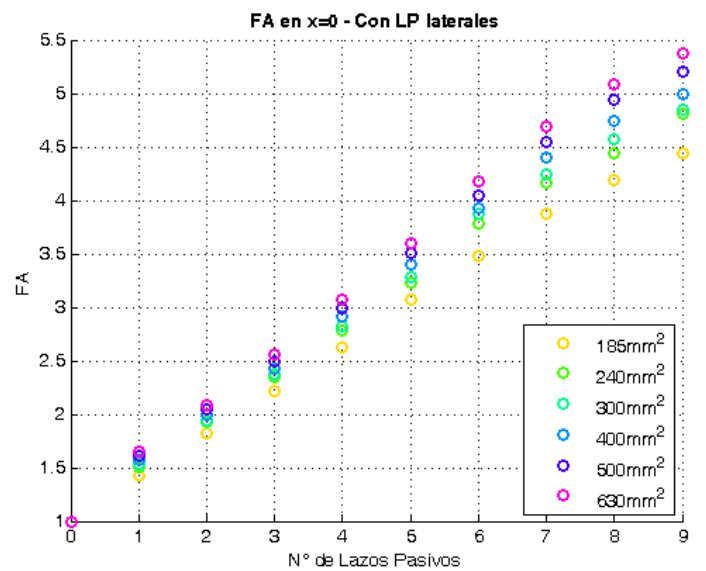

Fig. 103 - Valores de FA en $\mathrm{x}=0 \mathrm{a}$, considerando lazos pasivos a ambos lados de los conductores de fase, Caso 2 - Tabla XV.

A partir de los resultados obtenidos, se puede concluir que las secciones mayores, para los conductores de lazos pasivos, son las que arrojan mejores resultados. No obstante, la decisión sobre que sección utilizar tiene además un aspecto económico, ya que secciones más grandes significan costos superiores.

Otro aspecto importante a considerarse en la elección de la sección a utilizar, es evaluar cuales son las secciones típicas utilizadas por el propietario de la instalación, a la que se pretende aplicar la mitigación de campo magnético. A partir de las secciones disponibles, se estudia cual es la configuración que ofrece mejor desempeño.

\subsubsection{Configuración de los lazos pasivos}

En este punto se presentan distintas posibilidades de agrupamiento de los lazos pasivos, las configuraciones fueron planteadas en la Tabla XV. Dos de estas alternativas ya fueron presentadas en los incisos anteriores cuando se analizó la influencia del número de lazos pasivos y la sección de los conductores.

Para simplificar el problema, se considera una sola sección de conductores $\left(630 \mathrm{~mm}^{2}\right)$.

En el Anexo IX, se presentan los perfiles de campo magnético obtenidos y los correspondientes al FA (AIX-4).

Para analizar con más detalle las diferentes alternativas se realiza una comparación de los resultados en $\mathrm{x}=0$, que es el punto de mayor valor de campo magnético sin mitigación. En la Fig. 104 se comparan los valores de campo magnético y en la Fig. 105 se grafican los valores de FA. 


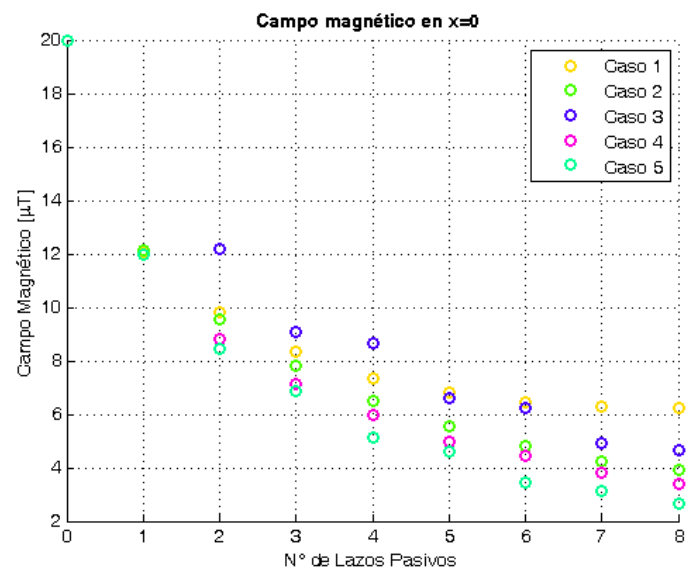

Fig. 104 - Valores de campo magnético en $\mathrm{x}=0$, Casos 1 a 5 - Tabla XV.

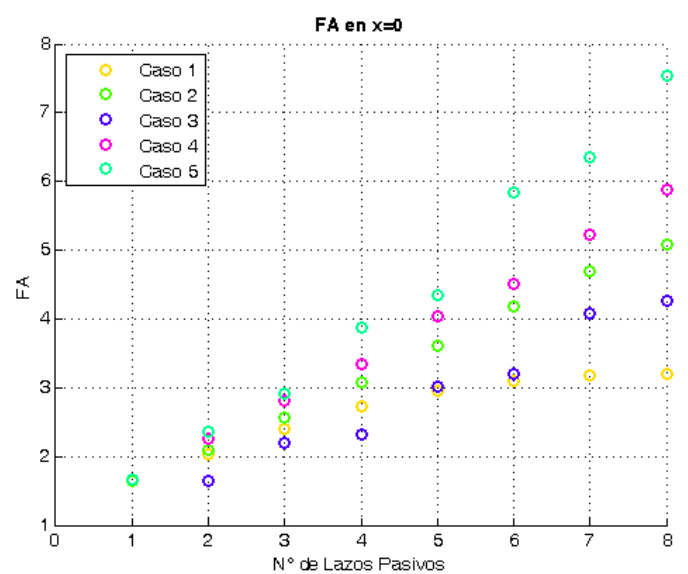

Fig. 105 - Valores de FA en $\mathrm{x}=0$, Casos 1 a 5 Tabla XV.

Analizando estas dos figuras es posible destacar algunas situaciones:

Caso 1: En esta situación en donde los conductores se disponen en un plano por encima de los conductores de fase, se logran importantes valores de atenuación empleando hasta seis lazos pasivos, a partir de aquí el campo magnético continúa reduciéndose pero los factores de reducción no son tan grandes, quedando en valores ligeramente superiores a 3 . Los últimos lazos pasivos se dispusieron en la parte central, lo que no reportó mayores beneficios.

La principal ventaja de esta configuración es la simple instalación, ya que permite obtener valores aceptables de FA, instalando los lazos pasivos en la parte superior de los conductores de fase.

Caso 2: Esta configuración, en donde los conductores de lazos pasivos se disponen a ambos lados de los conductores de fase, presenta muy buen desempeño, con el empleo de 8 lazos pasivos es posible reducir el valor de campo magnético en la zona de mayor valor hasta 5 veces. Si se compara con la configuración del Caso 1, su construcción es un poco más compleja. Los lazos pasivos son todos iguales, igual largo y ancho.

Caso 3: En este caso se consideran lazos pasivos dispuestos en dos planos equidistantes al plano formado por los conductores de fase, uno por encima y otro por debajo. Esta instalación posee los conductores en el plano inferior a mayor distancia de la zona de interés que los Casos 1 y 2, hasta el empleo de 6 conductores es la que presenta un peor desempeño. A partir de 6 conductores el FA es mayor que el del Caso 1.

Caso 4: En este caso se disponen los lazos pasivos en dos planos, un plano es el mismo en el que se disponen los conductores de fase y el otro plano está ubicado en la parte superior. Comparando los resultados obtenidos; se observa que esta configuración arroja mejores resultados que los anteriores. El máximo valore de FA es aproximadamente 6, para 8 lazos pasivos.

Caso 5: En este caso se disponen los lazos pasivos en el perímetro del plano formado por los conductores de fase, es una combinación de los casos 2 y 3. Con lazos en la parte superior, inferior y el lateral de los conductores de fase. Este arreglo permite obtener los mayores valores para el FA, 7,5 empleando 8 lazos. Esta configuración es la de más compleja instalación, ya que se debe disponer lazos pasivos en la parte inferior del empalme. 


\subsubsection{Distancia entre Lazos y conductores de fase}

Cuando se pretende analizar lo que ocurre al modificar la distancia entre conductores de lazos y conductores de fase, hay que tener en cuenta además cual será la distancia final entre los lazos y la zona de interés y su influencia el valor final de campo magnético.

Otro factor a considerarse es el incremento de la temperatura en los conductores de fase, que se produce debido a la presencia de corriente en los lazos pasivos.

Entre las principales características de un cable se destaca la "capacidad de transporte de corriente", es común encontrar en la bibliografía la referencia está utilizando la expresión correspondiente en ingles "rating".

Si se hace referencia a la "reducción de la capacidad de transporte de corriente", es común encontrar también la expresión en ingles "derating".

Las instalaciones deberían diseñarse para provocar el mínimo derating en los conductores de fase. Típicamente el valor de derating provocado por estas soluciones está entre 0 y 2 \% [ 46 ].

\subsubsection{Comentarios generales}

A continuación se realizan algunos comentarios que permiten destacar los principales aspectos a tener en cuenta en el diseño de una estrategia de mitigación con lazos pasivos.

La principal ventaja de este método es la simplicidad de su instalación, ya que se emplean conductores aislados de Baja Tensión. Para el montaje de los lazos sólo debe realizarse un buen empalme con un elemento de unión adecuado, esto no debería aportar valores de resistencia adicionales que incremente significativamente la resistencia total del lazo. El aislamiento debe ser convencional de baja tensión, debe considerarse un buen sellado de forma tal de no permitir el ingreso de humedad.

La configuración de más simple implementación es la que se disponen los lazos pasivos en un plano entre los conductores de fase y la zona de interés, ya que los lazos se incorporan a la instalación luego de que los conductores de fase se encuentren instalados.

La elección de la configuración dependerá del valor de atenuación requerido en cada caso y de la geometría de la instalación. Cuando se realizan empalmes en cables subterráneos, la instalación se realizan bajo la vereda o bajo calzada, por lo que se debe considerar el espacio disponible en la obra civil, entonces debe contemplarse el ancho y la profundidad a la que se pueden extender las instalaciones. A partir de estas dimensiones se debe evaluar cual será la opción que mejor se adapte.

En algunas circunstancias los empalmes se realizan en cámaras en lugar de directamente enterrados, entonces es posible acceder a toda la periferia del empalme, con lo que en estas situaciones se podría evaluar alguna de las alternativas más complejas de las planteadas.

Aquí se estudiaron secciones disponibles en catálogos de fabricantes de cables, pero podría evaluarse la utilización de secciones mayores, lo que disminuiría la resistencia de los lazos pasivos. El problema que se presenta es que sería necesario realizar un pedido especial de las mismas, con el costo que esto trae aparejado. Por otro lado grandes secciones presentan ciertas dificultades para su manipulación y armado de los lazos. Considerando estas cuestiones, prácticamente seria una solución utilizar conductores disponibles en el mercado y estudiar el número de lazos y configuración que mejor se adapte.

La elección de la sección a utilizar en definitiva es un aspecto económico a tener en cuenta, en las Fig. 106 y Fig. 107 se presentan gráficos para los Casos 1 y 2 respectivamente, en los 
que se comparan los valores de FA en función de la sección de conductores y el número de lazos pasivos.

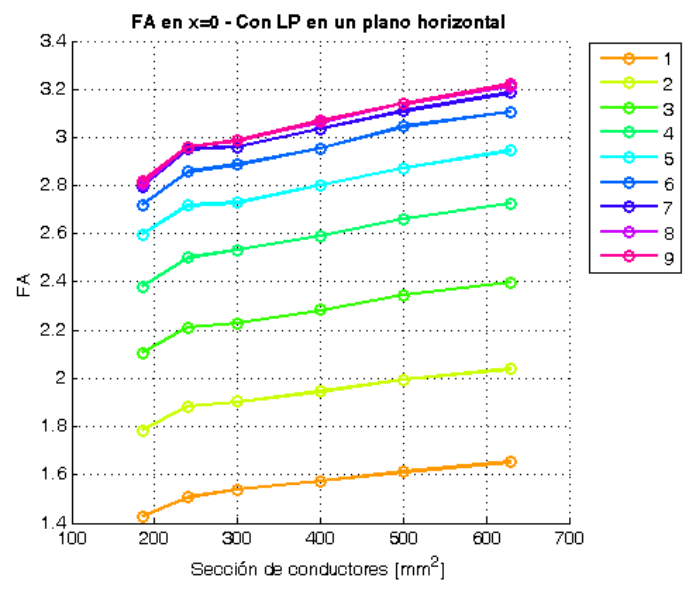

Fig. 106 - Valores de FA en $\mathrm{x}=0$, Caso 1 Tabla XV.

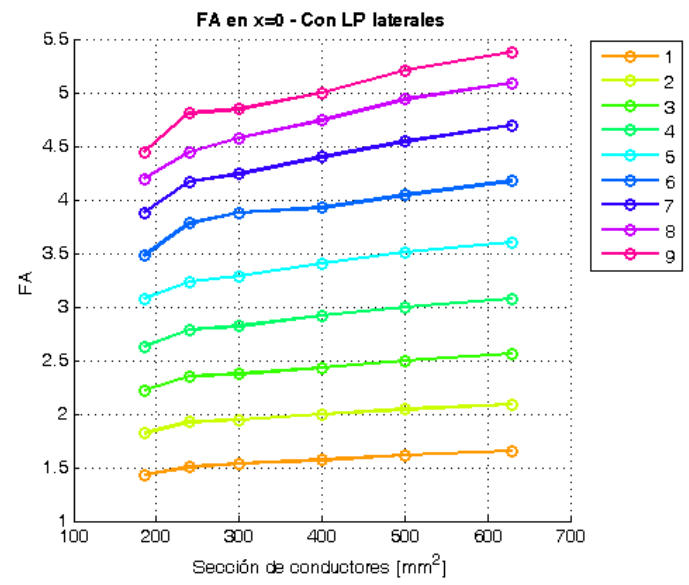

Fig. 107 - Valores de FA en $\mathrm{x}=0$, Caso 2 Tabla XV.

\subsection{Lazos pasivos en LAAT}

En este punto se evalúa la aplicación de Lazos Pasivos a Líneas Aéreas, para esto se consideran las LAAT de $500 \mathrm{kV}$ presentadas en el inciso 6.2. En la Fig. 108 se presenta una imagen con estos tipos constructivos de líneas, con una terna cada una. A la izquierda se aprecia la estructura tipo V-Arriostrada y a la derecha la torre Cross-Rope.

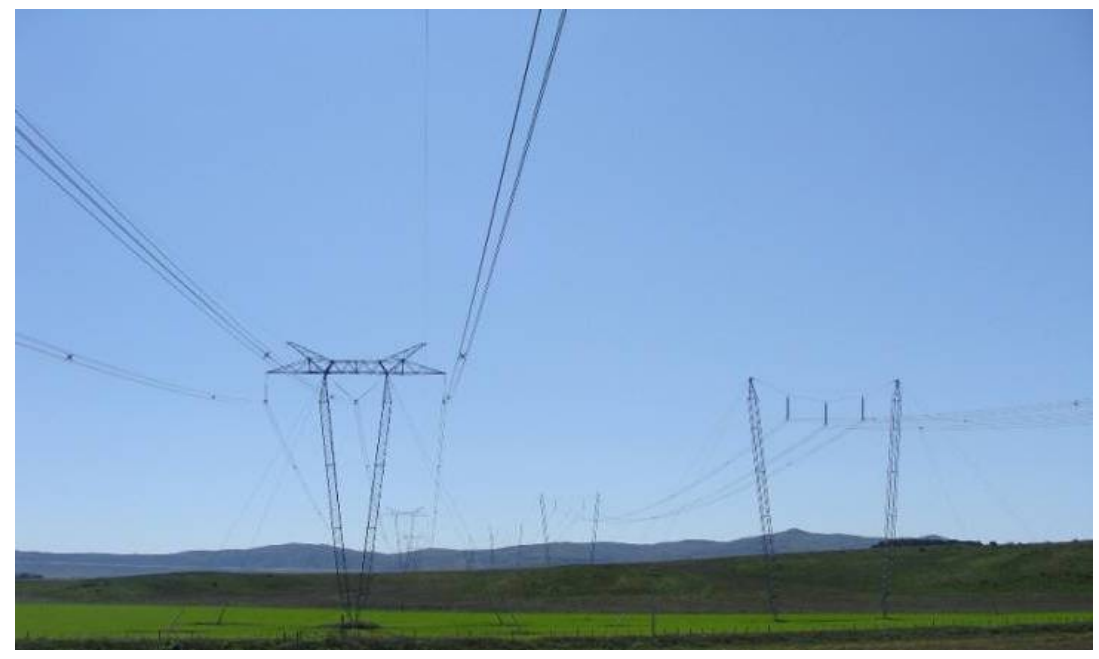

Fig. 108 - LAAT 500 kV V-Arriostrada y Cross-Rope.

La alternativa de utilización de lazos pasivos fue evaluada en todos los casos considerando para el lazo un conductor con una sección de $394 \mathrm{~mm}^{2}(0,131 \Omega / \mathrm{km})$, la longitud de lazo se consideró de $400 \mathrm{~m}$ y el ancho fue igual a la distancia entre los conductores externos.

Para los casos con la utilización de lazos pasivos se consideró que los mismos se dispusieran a la distancia mínima de seguridad para las líneas de $500 \mathrm{kV}$ en la República Argentina. Los lazos se ubican debajo de los conductores de fase externos (Esto se aprecia en las Fig. 109b) y Fig. 110b) los conductores de fase se indican en color negro y los de los lazos en azul), la distancia considerada entre estos y los conductores de los lazos se considero igual a la distancia entre los conductores de fase y la estructura.

En la Tabla XVI se identifican las configuraciones y casos planteados para este punto. 
Tabla XVI - Configuraciones de lazos pasivos consideradas - LAAT.

\begin{tabular}{|c|c|c|}
\hline Caso & Configuración & Observaciones \\
\hline 1 & Torre V-Arriostrada & Sin Lazos Pasivos \\
\hline 2 & Torre Cross-Rope & Sin Lazos Pasivos \\
\hline 3 & Torre V-Arriostrada & Con Lazos Pasivos \\
\hline 4 & Torre Cross-Rope & Con Lazos Pasivos \\
\hline
\end{tabular}

\subsubsection{Torre V - Arriostrada}

En la Fig. 109 se aprecian los resultados obtenidos para los "Casos 1 y 3", los resultados presentados en a) corresponden a la altura de medición para los conductores de fase de 18,3 m y la carga al momento de la medición fue de 133 A, en b) se presentan los resultados obtenidos con y sin la utilización de lazos pasivos y en c) se presenta el FA correspondiente, se aprecia que el máximo valor de FA es de 1,34, pero se da a una distancia de 12,6 m del centro de la línea.

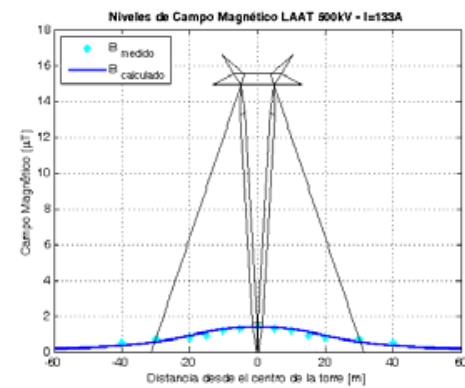

a) Mediciones I = $133 \mathrm{~A}$

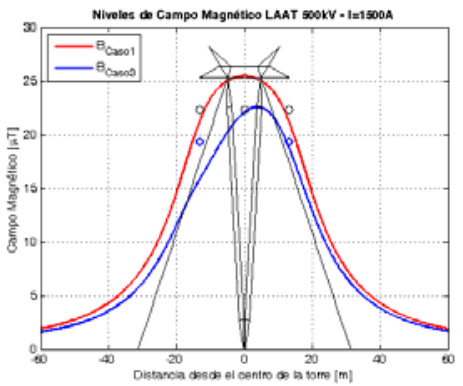

b) Calculo, $\mathrm{I}=1500 \mathrm{~A}$

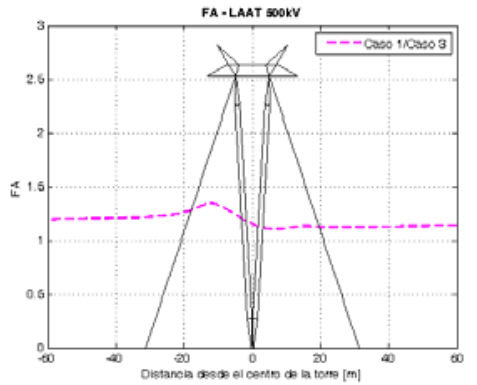

c) Caso 4 - FA

Fig. 109 - LAAT 1 x $500 \mathrm{kV}$ - Caso 4.

En el centro de la línea, en donde el valor de campo magnético es más alto, el valor obtenido de FA es de 1,15.

\subsubsection{Torre Cross-Rope}

En la Fig. 110 se aprecian los resultados obtenidos para los "Casos 2 y 4", los resultados presentados en a) corresponden a la altura de medición para los conductores de fase de 15,5 m y la carga al momento de la medición fue de 200 A, en b) se presentan los resultados obtenidos con y sin la utilización de lazos pasivos y en c) se presenta el FA correspondiente, se aprecia que el valor de atenuación obtenido en la zona central, donde el campo magnético es más grande, es inferior a 1,46.

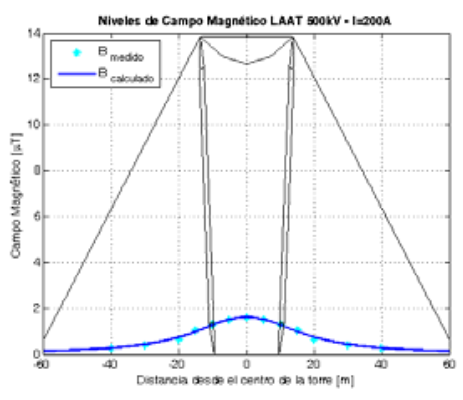

a) Mediciones I $=200 \mathrm{~A}$

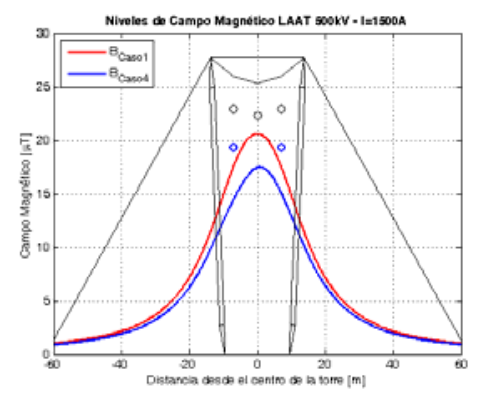

b) Calculo, $\mathrm{I}=1500 \mathrm{~A}$

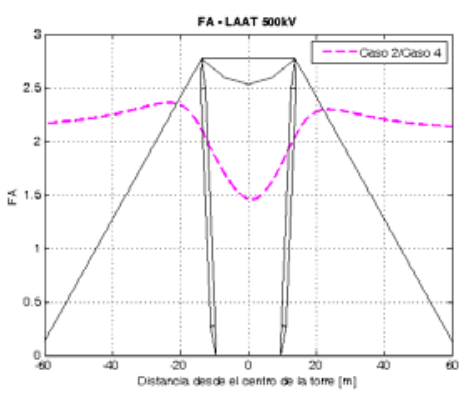

c) Caso 5 - FA

Fig. 110 - LAAT 1 x 500 kV - Caso 5 . 
Para zona alejadas del centro se obtienen mayores valores de FA, el mayor valor obtenido es de 2,36 a 22,8 m del centro de la línea.

En la Fig. 111 se presenta una comparación entre los factores de atenuación, se tomó como referencia la configuración del Caso 1, ya que la misma es la que presenta mayores valores de campo magnético. Se comparó con las demás alternativas analizadas.

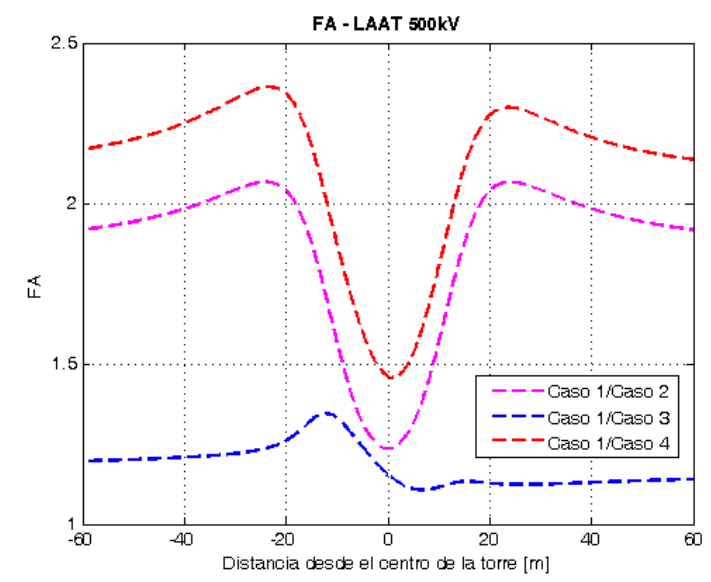

Fig. 111 - Comparación del FA LAAT 500 kV.

Para las Líneas simple terna de $500 \mathrm{kV}$ consideradas, se aprecia que la línea más compacta, "Caso 2", arroja mejores resultados, en comparación con el "Caso 1". A su vez si se utiliza un lazo pasivo, considerando la geometría del mismo y el módulo y fase de la corriente del lazo, es posible obtener una reducción substancial del campo magnético en una locación específica.

La utilización de lazos pasivos sería aplicable a situaciones en donde sea requerida una reducción local del campo magnético, en las cercanías de LAAT existentes. Para líneas nuevas podría considerarse la disposición de los conductores de fases a una mayor altura con respecto al terreno.

Para los casos de circuitos simples en configuración coplanar vertical, la utilización de lazos pasivos no es una alternativa eficiente, ya que todos los conductores de fase se encontrarían equidistantes del lazo, si el mismo estuviera centrado, por lo que la alternativa seria alejar la posición de la línea de la zona de interés. 


\section{Ejemplo de aplicación de la herramienta de cálculo desarrollada.}

Contar con una herramienta de cálculo que permita obtener los valores de campo magnético para diversas configuraciones de conductores de fase y conformación de lazos pasivos, permite explorar varias alternativas de diseño con el fin de evaluar aquella que mejor se adapte a las condiciones de requerimientos que se impongan y factibilidad de ejecución que se obtenga.

Se propone como ejemplo de aplicación, el tendido de CSAT según lo indicado en el inciso 5.3.1. La configuración geométrica se presenta en la Fig. 4 y los perfiles en la zona de ductos y fosa de empalmes, para una corriente de 1000 A, se presentan en la Fig. 112.

Se propone reducir los niveles de campo magnético en la zona de empalmes a valores similares a los que se obtienen en la zona de ductos.

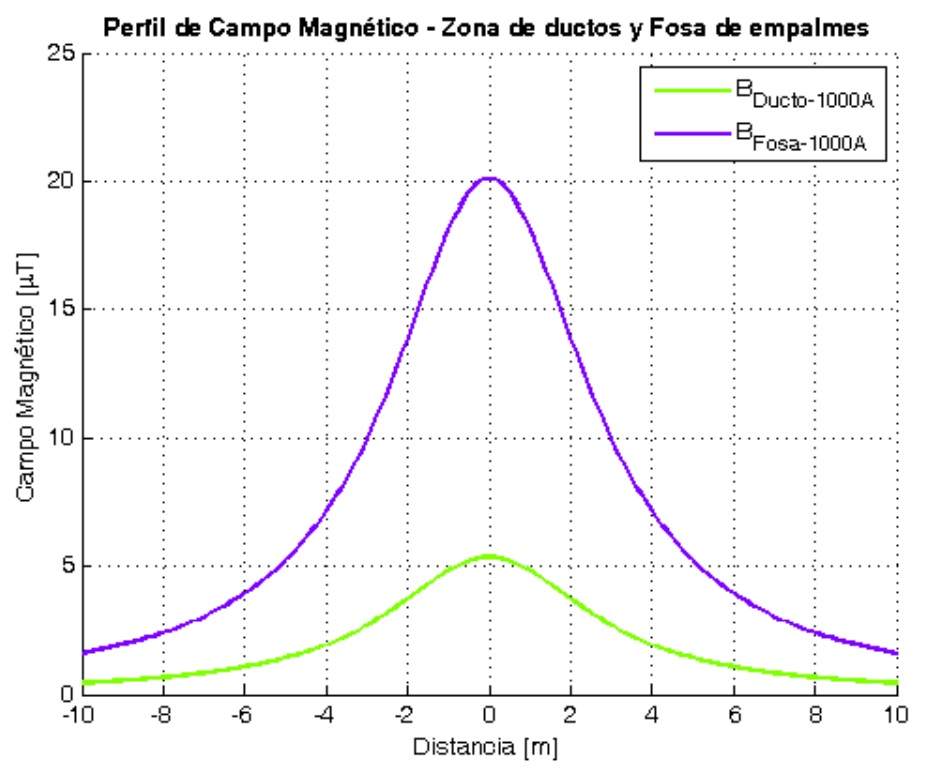

Fig. 112 - Perfiles de campo magnético, ejemplo del inciso 5.3.1 I=1000 A.

Si se considera el mayor valor de campo, este se presenta en la zona central $(\mathrm{x}=0)$, en la fosa dicho valor es de $20 \mu \mathrm{T}$ y en la zona de ductos es 5,3 $\mu \mathrm{T}$ ( $\mathrm{I}=1000 \mathrm{~A}$ ).

Entonces el objetivo sería reducir los valores de campo, en la Fosa de empalmes a un valor lo más próximo posible a 5,3 $\mu \mathrm{T}$, o lo que es equivalente obtener un valor de FA de 3,77.

Las alternativas de disposición de los lazos pasivos son los indicados en los Casos 1 a 5 de la Tabla XV.

Los valores de campo magnético calculados en el centro de la fosa, contemplando las diversas alternativas de disposición de los lazos pasivos, los cuales tienen conductores de $630 \mathrm{~mm}^{2}$, se presentaron en la Fig. 104.

En la Fig. 113 se reproducen los valores de FA (Fig. 105) y además se incluye en línea de trazos en color rojo el valor objetivo considerado para el FA $(3,77)$. 


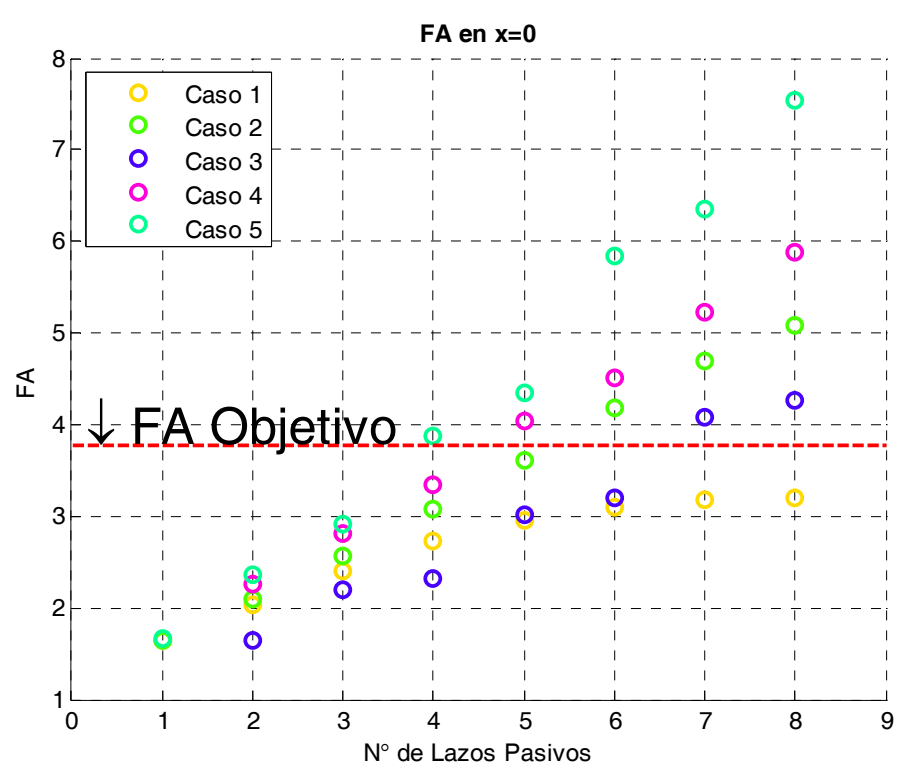

Fig. 113 - Valores de FA para diferentes alternativas de mitigación (Tabla XV), ejemplo del inciso 5.3.1 con $\mathrm{I}=1000 \mathrm{~A}$.

Analizando las diferentes alternativas, se observa que no todas las configuraciones representan una solución al problema con los requerimientos enunciados.

Con la configuración del Caso 1, se podría aspirar a valores de FA levemente superiores a 3, con 6 LP. A medida que se incorporan más lazos en la zona central no se obtienen mayores beneficios.

Para las demás configuraciones se observa una dependencia del número de LP considerados. El Caso 2 representa una solución a partir de considerar 6 lazos pasivos. El Caso 3 para el mismo número de LP (6) no logra cumplir con el objetivo, pero incrementando el número de lazos representa una posible solución. Algo similar ocurre con los Casos 4 y 5.

La configuración que requiere un menor número de LP es la que corresponde al Caso 5, ya que con 4 LP se logra un FA superior a 3,77.

Considerando el ejemplo planteado anteriormente puede concluirse que para seleccionar la alternativa que mejor se adapte a la instalación considerada, se debe realizar una comparación entre diferentes configuraciones, la más efectiva será aquella que permita alcanzar el objetivo con menores recursos.

Para concluir se puede considerar un orden de mérito para las configuraciones:

1. Caso 5, utilizando 4 LP como mínimo.

2. Caso 4, utilizando 5 LP como mínimo.

3. Caso 2, utilizando 6 LP como mínimo.

4. Caso 3, utilizando 7 LP como mínimo.

5. Caso 1, descartado.

El siguiente paso requiere un estudio de la factibilidad de instalación de la geometría seleccionada. Puede ocurrir que la solución óptima, desde el punto de vista de mitigación de campo magnético, no pueda aplicarse en la práctica por factores adicionales.

De hecho, suelen utilizarse los lazos pasivos ubicados en la parte superior de los conductores/empalmes (Caso 1), ya que no se suele tener acceso a otro tipo de instalación. En el caso de tendidos de cables en cámaras de empalmes ("manhole") permitiría realizar otro tipo de configuración (por ejemplo Caso 5). 
Asumiendo que sólo existe la posibilidad de implementar los lazos pasivos, con la disposición superior a los conductores de fase, se analizan otras variantes similares al Caso 1.

Resulta entonces de interés evaluar si, considerando la utilización de un número mayor de Lazos Pasivos, esta configuración puede aportar los valores de reducción requeridos.

A modo de ejemplo se consideran dos alternativas. La primera consiste en incorporar lazos cuyo ancho se incremente a partir de $1 \mathrm{~m}$ (Fig. 114). La otra consiste en incorporar lazos a partir de de un ancho de 0,4 m (Fig. 115).

La principal diferencia entre estas dos opciones es el ancho del lazo más interno, a partir de los cuales se van incorporando lazos hacia el exterior.

Para estos casos se considera conductores con una sección de $630 \mathrm{~mm}^{2}$ y una separación entre centros de los conductores de los lazos pasivos de $50 \mathrm{~mm}$.

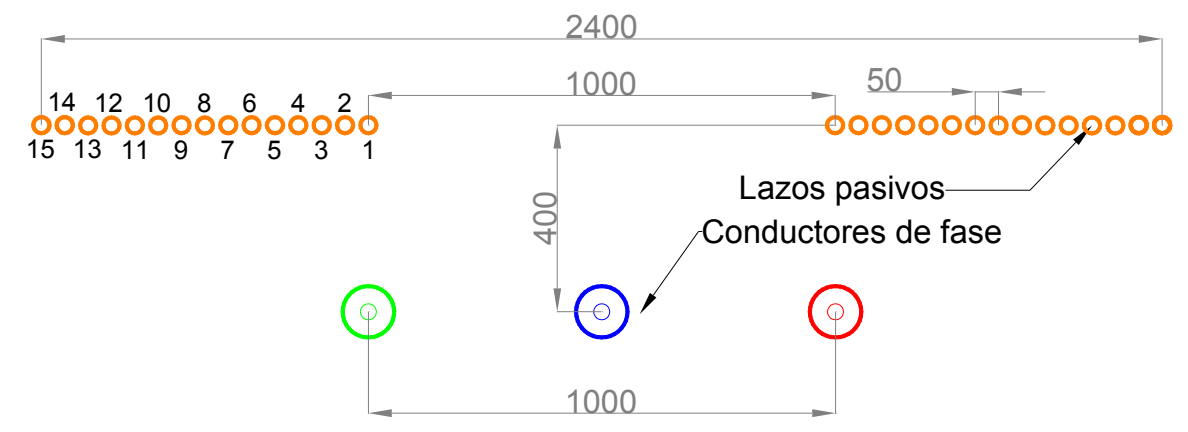

Fig. 114 - Variante del Caso 1 (LPH 1), ancho del LP interno $1 \mathrm{~m}$.

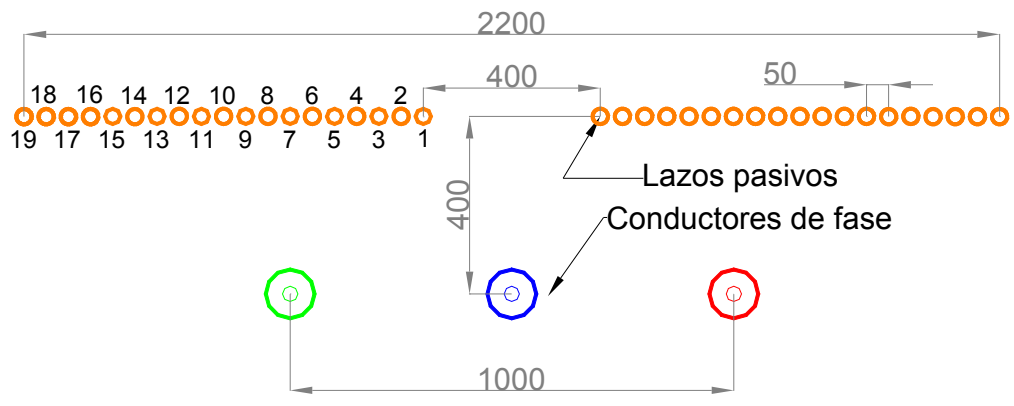

Fig. 115 - Variante del Caso 1 (LPH 2), ancho del LP interno 0,4 m.

En la Fig. 116 se presentan los valores de campo magnético, en función del número de lazos pasivos, que se obtienen en $\mathrm{x}=0$, punto de mayor nivel de campo magnético sin mitigación, que a su vez coincide con el conductor de la fase central. 


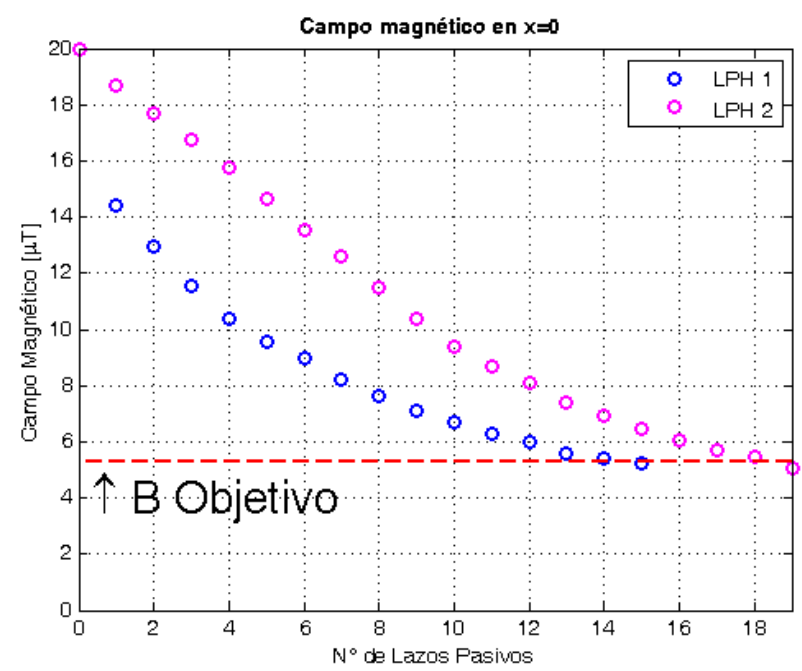

Fig. 116 - Valores de Campo magnético en función del número de lazos pasivos, Variantes del Caso 1, LPH 1 y 2. Sección de conductor de lazo $630 \mathrm{~mm}^{2}$.

En la Fig. 117 se presentan los valores correspondientes del FA, en función del número de lazos pasivos.

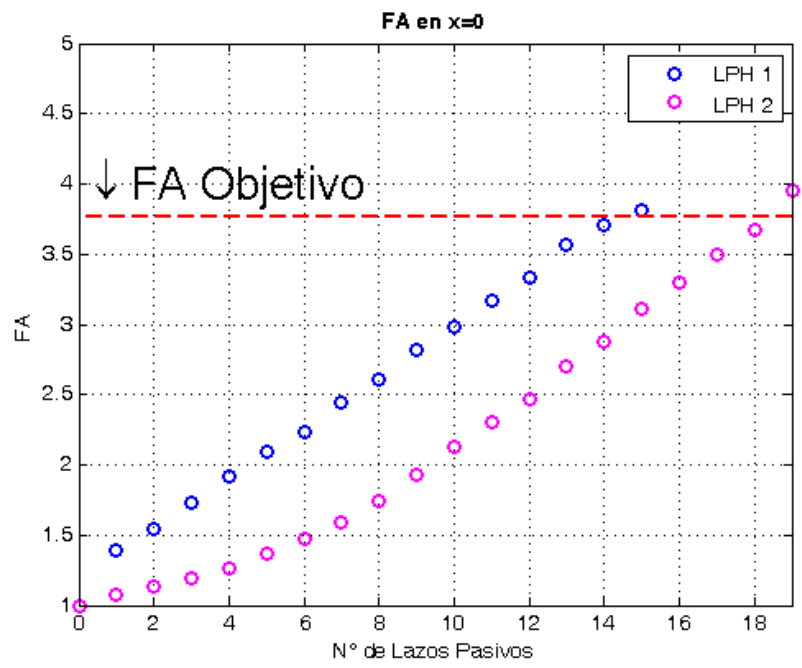

Fig. 117 - Valores de FA en función del número de lazos pasivos, Variantes del Caso 1, LPH 1 y 2. Sección de conductor de lazo $630 \mathrm{~mm}^{2}$.

Analizando los valores obtenidos de campo magnético y FA, se observa que si se parte de la utilización de lazos con anchos menores, ubicados en la zona central de la instalación, estos no presentan una influencia significativa.

Comparando entre las dos opciones analizadas, la identificada con LPH 1 resulta ser la más efectiva desde el punto de vista de niveles de atenuación de campo magnético y número de lazos empleados. Se alcanza el valor objetivo empleando un menor número de lazos pasivos. Con la opción LPH 1 se requieren 15 LP, mientras que con la LPH 2 se requieren 19 LP.

No obstante la configuración LPH 1 requiere un espacio mayor, el ancho del lazo pasivo externo es de 2, 4 m, mientras que en la configuración LPH 2 el ancho del lazo externo es de $2 \mathrm{~m}$.

Otro detalle a tener en cuenta es la longitud de cable requerida entre las dos opciones, en la configuración LPH 1 se requiere el empleo de 15 LP, lo que resulta en una longitud total de 
cable de $390 \mathrm{~m}$. La alternativa LPH 2, en la que se emplearían 19 lazos, requiere una longitud de cable de $494 \mathrm{~m}$.

La opción LPH 2, si bien ocupa menos espacio horizontal, requiere alrededor de $26 \%$ más de material.

Considerando la alternativa más económica, desde el punto de vista de la utilización de cables (LPH 1), se evalúa la adopción de una sección de conductor más pequeña, para 15 lazos. Empleando un conductor de sección $500 \mathrm{~mm}^{2}$, se obtiene un valor de campo magnético, en $\mathrm{x}=0$, de 5,3 $\mu \mathrm{T}$, o un FA de 3,77. Estos valores coinciden con los objetivo, por lo que la adopción de esta sección seria una alternativa aún más económica.

En la Fig. 118 se presentan los perfiles de campo magnético obtenidos para estas tres variantes. Además se incorpora el perfil sin la utilización de mitigación.

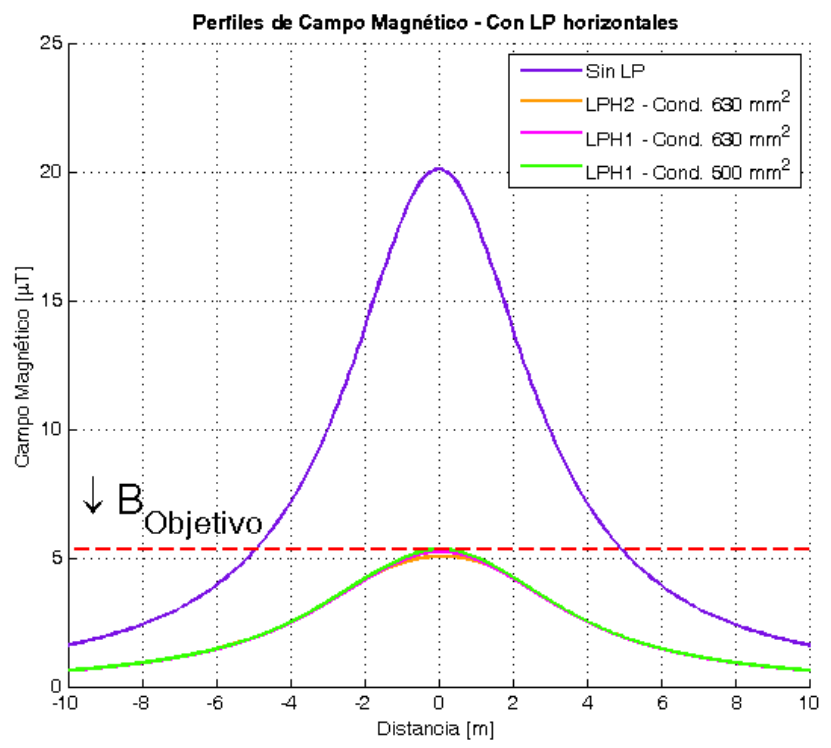

Fig. 118 - Perfiles de Campo Magnético obtenidos para las variantes del Caso 1, corriente $1000 \mathrm{~A}$.

Se destaca que la utilización de la herramienta desarrollada permite analizar diferentes alternativas y encontrar la que represente una mejor solución técnico-económica.

Es posible explorar diferentes configuraciones. Dentro de una misma configuración analizar diferentes alternativas, como ser el número de lazos pasivos, sección de conductor y diferentes separaciones entre los lazos. Esto permite optimizar el diseño de la mitigación y encontrar la configuración que mejor se adapte a la instalación a la que se le pretende aplicar esta técnica de mitigación.

Debe aclararse que si se quisiera implementar alguno de los arreglos considerados en este punto, debería realizarse un estudio complementario de calentamiento. Esto se debe al gran número de conductores que intervendrían en la mitigación y la posibilidad de que ellos provoquen un nivel de derrating considerable en los conductores de fase. 


\section{Consideraciones finales}

En este trabajo se estudiaron algunas posibilidades de la mitigación de campo magnético generado por líneas aéreas y cables subterráneos. En particular, se consideró la aplicación de lazos pasivos. Para ello se emplearon modelos que permiten obtener el campo magnético generado por las instalaciones eléctricas. Con estos modelos se desarrollaron una serie de módulos que permiten realizar cálculos de campo magnético y estimar los diferentes parámetros que intervienen en la implementación de lazos pasivos.

Las herramientas de cálculo desarrolladas, se utilizaron para evaluar diferentes posibilidades de aplicación de lazos pasivos y se implementaron modelos experimentales en laboratorio para comparar los resultados obtenidos por cálculos con mediciones. Los resultados obtenidos fueron satisfactorios.

Las configuraciones que permiten una mayor reducción de campo son aquellas cuyos conductores se disponen en el perímetro de los conductores de fase. Esta configuración tiene aplicación viable en cables subterráneos, cuando es posible acceder a todo el perímetro y disponer los lazos directamente enterrados

La configuración más simple de instalar, es la que dispone uno o más lazos en un mismo plano, ubicado entre los conductores de fase y la zona de interés. Esta alternativa es aplicable a cables subterráneos y a líneas aéreas. En el caso de cables subterráneos se implementan en un plano por encima de los conductores de fase, el proceso de instalación es relativamente sencillo. Para el caso de líneas aéreas se disponen en un plano inferior al de los conductores de fase, en este caso resulta compleja la utilización de múltiples lazos ya que existen cuestiones estructurales que deben ser consideradas.

La implementación de lazos pasivos en un solo plano presenta además la ventaja de que es posible realizar la implementación a instalaciones ya existentes, sin necesidad de modificar la configuración de los conductores de fase. Para el caso de líneas aéreas debe realizarse un estudio adicional que contemple los esfuerzos adicionales a los que se verían sometidas las estructuras de soporte.

Esta técnica es de gran utilidad en los casos en que se tiene una única terna y no existe la posibilidad de incrementar la distancia a la zona de interés. Tampoco es posible modificar la secuencia de energización, alternativa viable en cuando se tienen múltiples ternas.

Los niveles de reducción varían ya que se deben considerar donde se está analizando el grado de reducción de campo magnético, si es la zona de máximo valor de campo o si es una zona alejada.

Una ventaja importante, de la aplicación de lazos pasivos como estrategia de mitigación, es que no se requiere mantenimiento. En la etapa de montaje es necesario realizar al menos un empalme y el aislamiento correspondiente es de baja tensión para la aplicación a cables subterráneos. Con un buen aislamiento es posible evitar problemas de corrosión que podrían afectar a los conductores y el elemento de unión, este es un problema importante cuando se implementan estrategias de mitigación utilizando placas metálicas.

\subsection{Conclusiones}

A partir del objetivo principal del trabajo, se desarrollaron herramientas de cálculo bidimensionales que permiten realizar cálculos de campo magnético en Cables y Líneas Aéreas. Se implementaron además módulos de cálculo que permiten incorporar el efecto de los lazos pasivos en estas instalaciones. 
Se estudió la implementación de una estrategia de mitigación pasiva, que en caso de Cables Subterráneos arroja muy buenos resultados y ofrece ventajas desde el punto de vista técnico económico, respecto de otras técnicas de mitigación.

Para el caso de Líneas Aéreas, pueden obtenerse buenos resultados, pero la implementación resulta más compleja, ya que deben considerarse cuestiones vinculadas a las estructuras de soporte y distancias de aislamiento.

En líneas generales, si se considera una instalación a la que se pretende aplicar una estrategia de mitigación utilizando Lazos Pasivos, decidir cuál será la configuración que mejor se adapte, dependerá del nivel de mitigación de campo magnético requerido.

Es necesario definir un valor objetivo, si la instalación original genera valores de campo magnético que superen el requerido por la reglamentación vigente. El diseño se debe orientar a obtener valores de campo inferiores a los indicados por la normativa de aplicación. El valor máximo permitido por la normativa Argentina es de $25 \mu \mathrm{T}$

Otro criterio podría ser el planteado en el inciso 8, lograr que para CSAT los valores de campo en la zona de empalmes sean similares a los presentes en la zona de ductos.

La normativa Argentina [ 6 ] contempla además, la adopción de diseños teniendo en cuenta valores de campo magnético tan bajos como razonablemente alcanzables. En esta situación las configuraciones más simples de instalar y que representen menores costos permitirían reducir los valores de campo magnético.

Si existen diferentes configuraciones que permitan lograr el objetivo, una comparación entre las diferentes alternativas permitirá seleccionar la que represente una solución con menores recursos, esto incluye aspectos técnicos y económicos.

El trabajo realizado, permitió incorporar conocimientos sobre el comportamiento de los lazos pasivos y su empleo para la mitigación de campo magnético. Esta técnica posee un gran potencial de aplicación, considerando que en los centros urbanos se requieren cada vez más tendidos de cables subterráneos, para abastecer la creciente demanda de energía eléctrica.

Las herramientas de cálculo, permitirán continuar estudiando soluciones de mitigación de campo magnético utilizando lazos pasivos y además permiten realizar diseños o evaluar diferentes alternativas para futuras instalaciones.

\subsection{Propuesta de futuros trabajos}

En esta tesis se analizó la aplicación de placas de materiales ferromagnéticos y/o conductores para la mitigación de campo magnético en geometrías longitudinales.

Resulta de interés estudiar y evaluar la aplicación de estos materiales para mitigación de campo magnético en otro tipo de instalaciones vinculadas al sistema eléctrico, considerando las características de los diferentes materiales y diferentes configuraciones geométricas. 


\section{Referencias}

[1 ]: "Electrical wiring configurations and childhood cancer". Wertheimer N., Leeper E. Am. J. Epidemiol 1979; 109: 273-84.

[ 2 ]: "IARC Monographs on the evaluation of carcinogenic risks to humans. Non-Ionizing Radiation, Part 1: Static and Extremely Low-Frequency (ELF) Electric and Magnetic Fields". Volume 80. World Health Organization - International Agency for Research on Cancer 2002.

[ 3 ]: "ICNIRP guidelines for limiting exposure to time-varying electric and magnetic fields (1hz-100khz). Published in: health physics 99(6):818-836; 2010.

[4]: "ICNIRP guidelines for limiting exposure to time-varying electric, magnetic and electromagnetic fields (up to300Ghz). Published in: health physics 74(4):494-522; 1998.

[ 5 ]: Hoja Informativa $\mathrm{N}^{\circ}$ 263. Campos electromagnéticos y salud pública. Campos de frecuencia extremadamente baja y el cáncer. OMS EMF International Project. Octubre 2001.

[ 6 ]: Resolución SE 77/98, Secretaría de Energía de la Nación (Argentina). Boletín Oficial $\mathrm{N}^{\circ} 28.8591$ a Sección, 18 de marzo de 1998.

[ 7 ]: “Exposición a los Campos Electromagnéticos Estáticos y de Baja Frecuencia, Efectos Biológicos y Consecuencias en la Salud (0-100 kHz)". ICNIRP 13/2003. ISBN 9789972-2947-3-0. INICTEL-UNI, 2008.

[ 8 ]: "A Variety of Frequencies: transformer testing at the Pittsfield plant". Blalock, T.J. Power and Energy Magazine, IEEE Volume: 8, Issue: 4. DOI: 10.1109/MPE.2010.937132 Publication Year: 2010, Page(s): 75 - 88. IEEE JOURNALS \& MAGAZINES.

[ 9 ]: "Eyewitness to dc history". Lobenstein, R.W.; Sulzberger, C. Power and Energy Magazine, IEEE Volume: 6, Issue: 3. DOI: 10.1109/MPE.2008.920421 Publication Year: 2008, Page(s): 84 - 90 IEEE JOURNALS \& MAGAZINES..

[ 10 ]: IEEE Std 644TM-1994 (R2008), "IEEE Standard Procedures for Measurement of Power Frequency Electric and Magnetic Fields From AC Power Lines".

[11]: Cigré Technical Brochure 373 "Mitigation Techniques of power-frequency magnetic fields originated from electric power systems". WG C4.204, February 2009.

[12]: Handbook of Shielding Principles for Power System Magnetic Fields. Volume 1: Introduction and Application. Electric Power Research Institute, Palo Alto, California, 1994.

[ 13 ]: IEEE Std. C95.6TM - 2002, "IEEE Standard for Safety Levels with Respect to Human Exposure to Electromagnetic Fields, $0-3 \mathrm{kHz}$ ".

[ 14 ]: Directiva 2004/40/CE del Parlamento Europeo y del Consejo sobre las disposiciones mínimas de seguridad y de salud relativas a la exposición de los trabajadores a los riesgos derivados de los agentes físicos (campos electromagnéticos) (decimoctava Directiva específica con arreglo al apartado 1 del artículo 16 de la Directiva 89/391/CEE). 29 de abril de 2004. 
[ 15 ]: Monograph No. 238 "Extremely Low Frequency Fields Environmental Health Criteria". ISBN: 978924157238 5. (C) World Health Organization 2007

[16]: Nota descriptiva $N^{\circ} 322$. Campos electromagnéticos y salud pública. Exposición a campos de frecuencia extremadamente baja. OMS. junio de 2007

[ 17 ]: "ICNIRP guidelines on limits of exposure to static magnetic fields". Published in: health physics 96(4):504-514; 2009.

[ 18 ]: Ley N 19587/72 "Ley de higiene y seguridad en el trabajo". Publicada en B.O. el 28 de abril de 1972.

[19]: Resolución 295 / 2003. Ministerio de trabajo, empleo y seguridad social. Apruébanse especificaciones técnicas sobre ergonomía y levantamiento manual de cargas, y sobre radiaciones. Modificación del Decreto 351/79. Déjase sin efecto la Resolución 444/91 MTSS. Bs. As., 10/11/2003

[ 20 ]: Reglamento Técnico de Instalaciones Eléctricas RETIE. Quinta Actualización 2008 Resolución número 180398 del 7 de abril de 2004 por la cual se expide el Reglamento Técnico de Instalaciones Eléctricas - RETIE y se incluyen las modificaciones previstas en la resolución número la resolución número 181294 del 6 de agosto de 2008.

[ 21 ]: Resolução Normativa № 398, Agência Nacional De Energia Elétrica - ANEEL, 23 DE MARÇO DE 2010.

[ 22 ]: NRPB vol 15 N$^{\circ} 2$ 2004. "Advice on Limiting Exposure to Electromagnetic Fields (0$300 \mathrm{GHz}$ " Documents of the NRPB. Adopted "in the terms of the EU Recommendation" by letter from Government to NRPB 22 July 2004.

[ 23 ]: "Legge quadro sulla protezione dale esposizioni a campi elettrici, magnetic ed elettromagnetici”, Legge 22 febbraio 2001, n. 36. GU n. 55 del 7 marzo 2001.

[ 24 ]: "Fissazione dei limiti di esposizione, dei valori di attenzione e degli obiettivi di qualità per la protezione della popolazione dalle esposizioni ai campi elettrici e magnetici alla frequenza di rete $(50 \mathrm{~Hz})$ generati dagli elettrodotti", Decreto del Presidente del Consiglio dei Ministri 8 luglio 2003, G.U. n. 200 del 29 agosto 2003.

[ 25 ]: ONIR 814.710 "Ordinance relating to Protection from Non-Ionising Radiation (ONIR)". Federal Office of Environment (BAFU/FOEN). 01 February 2000.

[26]: Curso de postgrado compatibilidad de instalaciones con el ambiente. Facultad de Ingeniería. Universidad Nacional de La Plata. Dictado por: P. L. Arnera, M. B. Barbieri, D. A. Esteban y P. E. Issouribehere. Septiembre de 2009.

[ 27 ]: Transmissao de Energia eletrica. Rubens Dario Fuchs. Livros Técnicos e Cientificos Editora S.A. 1979.

[ 28 ]: IEC 61786:1998, "Measurement of low-frequency magnetic and electric fields with regard to exposure of human beings-Special requirements for instruments and guidance for measurements".

[ 29 ]: "Blindaje De Campo Magnético En Instalaciones Eléctricas". Carlos Wall, Beatriz Barbieri, Daniel Esteban, Patricia Arnera, Nicolás A. Casco(*). Instituto de Investigaciones Tecnológicas para Redes y Equipos Eléctricos (IITREE-LAT). Facultad de Ingeniería - Universidad Nacional de La Plata. (*)Instituto Argentino de Radioastronomía (IAR). IV Conferencia Internacional de Electromagnetismo Aplicado, CNEA 2011, 15 al 18 de Marzo del 2011, Santiago de Cuba, Cuba.

[ 30 ]: CIGRÉ TB 320 “Characterisation of ELF Magnetic Fields”, WG C4.205, april 2007 
[ 31 ]: Análisis, cálculo Y técnicas de mitigación de campos magnéticos creados por líneas eléctricas de alta tensión. Tesis doctoral. Pedro Luis Cruz Romero. Ingeniero Industrial. Escuela de Ingenieros de Sevilla. Sevilla, Septiembre de 2000.

[ 32 ]: "Requirements for Power Line Magnetic Field Mitigation Using a Passive Loop Conductor". K. Yamazaki, T. Kawamoto and H. Fujinami, IEEE Trans. on Power Delivery, vol. 15, No. 2, pp. 646-651, April 2000.

[ 33 ]: "Mitigation of Magnetic Field near Power Lines". Memari A.R., Janischewskyj W., IEEE Transactions on Power Delivery. Vol. 11, No. 3, pp. 1577-1586, July 1996

[ 34 ]: Reduction of magnetic fields from transmission lines using passive loops. B. Shperling, l. Menemenlis-Hopkins, B. Fardanesh B. Clairmont, D. Childs Cigré 1996 : 36-103

[ 35 ]: "Principios de Electrotecnia 3, vol. III". A. V. Netushil, K. M. Polivanov, Grupo Editor de Buenos Aires, 1980.

[ 36 ]: Electromagnetic Transients Program (EMTP). Reference Manual. Dommel. H.W., 4427 West 6th Avenue, Vancouver, Canada, Agosto 1986

[ 37 ]: "Series-capacitor Compensated Shield Scheme for Enhanced Mitigation of Transmission Line Magnetic Fields". Walling R.A., Paserba J.J., Burns C.W., IEEE Transactions on Power Delivery. Vol. 8, No. 1, pp. 461-469, Enero 1993.

[ 38 ]: "Implementation of a tool for magnetic field mitigation using passive loops". C. A. Wall, P. L. Arnera, M. B. Barbieri. IEEE T\&D LA Sixth IEEE/PES, 3-5 September 2012, Montevideo, Uruguay.

[ 39 ]: "Development of software for magnetic field calculation and mitigation using passive loops". C. A. Wall, P. L. Arnera, M. B. Barbieri. 3rdCigré International Colloquium on Electric and Magnetic Fields at Extremely Low Frequencies (EMF-ELF 2013), 15-16 October 2013, Nara, Japan.

[ 40 ]: “Opera 3D user manual”. Vector Field Ltd. Kindlington, OX5 1JE, England 2005.

[41]: "Passive loops technique for electromagnetic fields mitigation: applications and theoretical considerations". Paolo Maioli, Ernesto Zaccone. JICABLE 2007, Versailles, France, June 24 to 28th.

[ 42 ]: "Mitigación de campos magnéticos en líneas subterráneas de potencia mediante el empleo de lazos pasivos". P. Cruz, J. Hoeffelman y J. C. del Pino. IEEE Latin America Transactions, VOL. 6, NO. 1, March 2008.

[ 43 ]: "Techniques for the mitigation of the magnetic field in HV insulated lines". P. Maioli. 2010 IEEE International Symposium on Electrical Insulation (ISEI).

[ 44 ]: Guía Técnica de Prysmian - Selección y Dimensionamiento de Conductores de Baja Tensión. Edición 2007.

[ 45 ]: Res. ENRE 1724/98, ANEXO: instrucciones para la medición de campos eléctrico y magnético en sistemas de transporte y distribución de energía eléctrica. Boletín Oficial $\mathrm{n}^{\circ} 29.038$, lunes 7 de diciembre de 1998, pp. 25-26.

[ 46 ]: CIGRÉ TB 559 "Impact of EMF on current ratings and cable systems", WG B1.23, december 2013. 


\section{Anexo I}

Mecanismos de acoplamiento entre el campo magnético y el cuerpo Análisis de la IARC

Definiciones - ICNIRP 2010

Fundamentación de las líneas de orientación - ICNIRP 2010

Directrices para limitar la exposición a los CEM

Limitaciones básicas y niveles de referencia

Niveles de referencia de las principales guías a nivel internacional 


\section{AI-1. Mecanismos de acoplamiento entre el campo magnético y el cuerpo}

A continuación se mencionan los diferentes mecanismos de interacción entre el campo magnético y el cuerpo, indicados en la guías publicada por ICNIRP en el año 2010.

Para los campos magnéticos, la permeabilidad del tejido es igual a la del aire, por lo que el campo en los tejidos es igual al campo externo. El cuerpo de los humanos y animales no perturba significativamente el campo magnético externo.

La principal interacción de los campos magnéticos es la inducción de campos eléctricos y las corrientes asociadas en los tejidos. Los campos magnéticos pueden también ser inducidos por el movimiento en un campo magnético estático. Las principales características de dosimetría para la exposición de los seres humanos a campo magnético de baja frecuencia incluyen:

- para una determinada intensidad y orientación del campo magnético se inducen mayores campos eléctricos en cuerpos de personas con dimensiones mayores, dado que los posibles circuitos conductores cerrados son más grandes;

- el campo eléctrico y la corriente inducida depende de la orientación del campo magnético externo con relación al cuerpo. Normalmente, los campos inducidos en el cuerpo son mayores cuando el campo está alineado entre el frente y la parte posterior del cuerpo, pero para algunos órganos, los valores más altos corresponden a diferentes alineamientos de campo;

- campos eléctricos más débiles son inducidos por un campo magnético orientado a lo largo del eje principal del cuerpo;

- la distribución del campo eléctrico inducido se ve afectada por la conductividad de diversos órganos y tejidos.

\section{AI-2. Análisis de la IARC}

En el Vol. 80 de la IARC se realiza una clasificación de agente, mezcla o circunstancia de exposición, respecto a la posibilidad que estos generen cáncer. La categorización de un agente, mezcla o la circunstancia de exposición es una cuestión de juicio científico, lo que refleja la fuerza de la evidencia derivada de estudios en humanos y en animales de experimentación y de otros datos relevantes.

Grupo 1: el agente (o mezcla) es carcinogénico en seres humanos. La circunstancia de la exposición constará que son carcinógenos para los seres humanos. Esta categoría se utiliza cuando hay evidencia suficiente de carcinogenicidad en humanos. Excepcionalmente, un agente (mezcla) puede ser colocado en esta categoría cuando la evidencia de carcinogenicidad en humanos es menos que suficiente, pero es suficiente la evidencia de carcinogenicidad en animales de experimentación y evidencia contundentes en humanos expuestos que el agente (mezcla) actúa mediante un mecanismo importante de carcinogenicidad.

Grupo 2: Esta categoría incluye agentes, mezclas y circunstancias de exposición que, en un extremo, el grado de evidencia de carcinogenicidad en seres humanos es casi suficiente, así como aquellos para los que, en el otro extremo, no hay datos en humanos, pero hay evidencia de carcinogenicidad en animales de experimentación. Los agentes, mezclas y circunstancias de exposición se asignan al grupo 2A (probablemente cancerígeno para los seres humanos) o el grupo 2B (posiblemente carcinógeno para los humanos) sobre la base de la evidencia epidemiológica y experimental de la carcinogenicidad y otros datos relevantes. 
Grupo 2A: el agente (mezcla) es probablemente carcinógeno para los humanos. La circunstancia de la exposición constará de las exposiciones que son probablemente carcinogénicos en seres humanos. Esta categoría se utiliza cuando existe limitada evidencia de carcinogenicidad en humanos y suficiente evidencia de carcinogenicidad en animales de experimentación. En algunos casos, un agente (mezcla) se pueden clasificar en esta categoría cuando es inadecuada la evidencia de carcinogenicidad en humanos, y suficiente la evidencia de carcinogenicidad en animales de experimentación y pruebas contundentes de que la carcinogénesis es mediada por un mecanismo que opera también en los seres humanos. Con carácter excepcional, un agente, mezcla o la circunstancia de exposición se pueden clasificar en esta categoría únicamente sobre la base de limitada evidencia de carcinogenicidad en humanos.

Grupo 2B: el agente (mezcla) es posiblemente carcinogénico en seres humanos. La circunstancia de la exposición constará de las exposiciones que son posiblemente carcinogénicos en seres humanos. Esta categoría se utiliza para los agentes, mezclas y circunstancias de exposición para la que es limitada la evidencia de carcinogenicidad en humanos y evidencia menos que suficiente de carcinogenicidad en animales de experimentación. También se puede utilizar cuando es inadecuada la evidencia de carcinogenicidad en humanos, pero es suficiente la evidencia de carcinogenicidad en animales de experimentación. En algunos casos, un agente, mezcla o la circunstancia de exposición es inadecuada la evidencia de carcinogenicidad en humanos, y menos que suficiente la evidencia de carcinogenicidad en animales de experimentación, junto con el soporte de evidencias de mecanismos y datos pertinentes, que permiten ser colocados en este grupo.

Grupo 3: El agente (mezcla o circunstancia de exposición) no es clasificable en cuanto a carcinogenicidad en seres humanos. Esta categoría se utiliza más comúnmente para los agentes, mezclas y circunstancias de exposición para que las pruebas de carcinogenicidad en humanos es inadecuada e inadecuada o limitada en animales de experimentación.

Excepcionalmente, los agentes (mezclas) para los que la evidencia de carcinogenicidad en humanos es inadecuada, pero suficiente en animales de experimentación se puede colocar en esta categoría cuando hay fuerte evidencia de que el mecanismo de carcinogenicidad en animales de experimentación no funciona en los seres humanos.

Los agentes, mezclas y circunstancias de exposición que no se encuentran en ningún otro grupo también se incluyen en esta categoría.

Grupo 4: el agente (o mezcla) es probablemente no carcinógeno para los seres humanos. Esta categoría se utiliza para los agentes o mezclas para las que hay evidencia que sugiere ausencia de carcinogenicidad en humanos y en animales de experimentación. En algunos casos, los agentes o mezclas para las que es inadecuada la evidencia de carcinogenicidad en humanos pero la evidencia que sugiere ausencia de carcinogenicidad en animales de experimentación, y firmemente apoyado por una amplia gama de otros datos relevantes, se pueden clasificar en este grupo.

En función de los grupos anteriores, la IARC clasificó a los campos magnéticos de FEB como "posiblemente cancerígenos para los seres humanos" (2B) a partir de la evaluación de estudios publicados que concluyó así:

- Hay evidencia limitada en los seres humanos para la carcinogenicidad de los campos magnéticos de frecuencia extremadamente baja en relación con la leucemia infantil. La evidencia limitada significa que los estudios que establecen una relación causal 
entre la exposición al agente y el cáncer humano, no ofrecen elementos suficientes para descartar que los resultados sean la consecuencia de deficiencias metodológicas en el diseño o el análisis estadístico (probabilidad, error y variables de confusión).

- Hay inadecuada evidencia en los seres humanos para la carcinogenicidad de los campos magnéticos de frecuencia extremadamente baja en relación con otros tipos de cáncer.

- Hay inadecuada evidencia en los seres humanos para la carcinogenicidad de los campos eléctrico y magnético estáticos y los campos eléctricos de frecuencia extremadamente baja.

- Hay inadecuada evidencia en animales de experimentación para la carcinogenicidad de los campos magnéticos de frecuencia extremadamente baja.

- No hay datos relevantes para la carcinogenicidad de los campos eléctrico y magnético estáticos y los campos eléctricos de frecuencia extremadamente baja en animales de experimentación.

- La evaluación global de los campos magnéticos de frecuencia extremadamente baja indica que son posiblemente carcinogénicos para los seres humanos (Grupo 2B), en relación a la leucemia infantil.

- Los campos eléctrico y magnético estáticos y los campos eléctricos de frecuencia extremadamente baja son no clasificables en cuanto a su carcinogenicidad en seres humanos (Grupo 3).

\section{AI-3. Definiciones - ICNIRP 2010}

De la publicación efectuada por la ICNIRP en el año 2010 [ 3 ] se extraen las algunas definiciones que resultan de interés.

Sistema nervioso central (CNS): es la parte del sistema nervioso de los vertebrados que consiste en el cerebro y la médula espinal, pero no incluye los nervios periféricos.

Magnetofosfenos: Sensación visual de puntos de luz causados por las corrientes eléctricas inducidas que estimulan la retina.

Radiaciones No Ionizantes (RNI): Incluye todas las radiaciones y los campos del espectro electromagnético que generalmente no tienen suficiente energía para ionizar la materia. Se caracterizado por presentar una energía por fotón inferior a aproximadamente $12 \mathrm{eV}$, que es equivalente a longitudes de onda superiores a $100 \mathrm{~nm}$ y frecuencias inferiores a $3 \times 10^{15} \mathrm{~Hz}$.

Público en general: El termino público en general se refiere a toda la población, incluye a las personas de todas las edades y de diferente estado de salud, y esto incluye en particular los grupos o personas vulnerables, como los débiles, los trabajadores de edad avanzada, embarazadas, bebés y niños pequeños

Exposición del público en general: Toda exposición a campos de baja frecuencia recibida por los miembros del público en general. Esta definición excluye a la exposición ocupacional y exposición médica

En un ambiente controlado $u$ ocupacional, se consideran las exposiciones a los CEM experimentadas por individuos como resultado del desarrollo de las tareas asignadas en actividades laborales, estas personas conocen los niveles de exposición a los que se encuentran sometidos.

Cuando se hace referencia a la exposición del público en general, se aborda la problemática de todos aquellos ambientes de exposición, exceptuando las exposiciones ocupacionales o exposiciones durante procedimientos médicos. 
Exposición médica: es la exposición de una persona a campos de baja frecuencia recibidos como un paciente sometido a diagnóstico médico, o a un tratamiento médico reconocido, o como voluntario en una investigación médica.

Exposición ocupacional: Toda exposición a CEM de individuos como consecuencia de la ejecución de sus actividades profesionales diarias.

Niveles de referencia: Los valores eficaces y pico de campos eléctricos y magnéticos, y de corrientes de contacto a los que una persona puede estar expuesto sin sufrir ningún efecto adverso y con factores de seguridad aceptables. Los niveles de referencia para la exposición eléctrica y magnética se pueden superar si se demuestra que no se superen la restricción básica.

Sin embargo, se pueden utilizar parámetros prácticos o marcadores para determinar el cumplimiento de las restricciones básicas.

Umbral: Es el nivel de un estímulo que marca el límite entre una respuesta y no respuesta.

\section{AI-4. Fundamentación de las líneas de orientación - ICNIRP 2010}

En sus directrices ICNIRP aborda los efectos agudos y crónicos sobre la salud y tiene en cuenta los desarrollos dosimétricos realizados hasta el 2010.

\section{Efectos agudos}

Existen varios efectos agudos debidamente comprobados como resultado de la exposición del sistema nervioso a los campos electromagnéticos (CEM) de baja frecuencia: la estimulación directa de los nervios y el tejido muscular y la inducción de fosfenos en la retina. También hay evidencia científica indirecta de que las funciones del cerebro, tales como el procesamiento visual y la coordinación motora pueden verse afectados temporalmente por campos eléctricos inducidos. Todos estos efectos tienen umbrales por debajo de los cuales no se producen y pueden ser evitados si se respetan las limitaciones básicas sobre los campos eléctricos inducidos en el cuerpo.

A raíz de las recomendaciones formuladas en relación con las directrices sobre los límites de exposición a campos electromagnéticos estáticos (ICNIRP 2009), la ICNIRP considera que hay circunstancias profesionales en las que, con el asesoramiento y la formación adecuada, es razonable que los trabajadores debidamente informados y que acepten esta situación, sientan efectos temporales como la inducción de fosfenos en la retina y la posibilidad de alteración reducida en algunas funciones cerebrales, ya que se piensa que estos efectos no producen efectos patológicos sobre la salud o a largo plazo. La exposición de todas las partes del cuerpo en tales circunstancias debe limitarse, para evitar la estimulación de las fibras nerviosas mielínicas del sistema nervioso central y periférico. La ICNIRP hace notar el margen relativamente estrecho entre el umbral de percepción de los nervios periféricos y el umbral del dolor. Para ambos tipos de nervios, los umbrales alcanzan hacia arriba 1 a $3 \mathrm{kHz}$ debido a las muy pequeñas constantes de tiempo de las membranas resultantes de la mielinización e inferiormente sobre $10 \mathrm{~Hz}$ a causa de una lenta adaptación a los estímulos de despolarización.

Evitar fosfenos en la retina debe brindar protección contra posibles efectos sobre la función cerebral. Los umbrales de los fosfenos están alrededor de los $20 \mathrm{~Hz}$ y aumenta rápidamente para frecuencias superiores e inferiores, que se cruzan con los umbrales de estimulación del sistema nervioso periférico y central, en el punto donde se aplica el umbral para la estimulación del sistema nervioso periférico. Para los trabajadores que no poseen la formación adecuada, pueden no ser conscientes de este problema y que no pueden controlar su nivel de exposición, la limitación básica se encuentra en el umbral de los fosfenos, con el 
fin de evitar los efectos transitorios potencialmente perjudiciales. Para los miembros del público en general, se aplica el límite correspondiente a los fosfenos considerando un factor de reducción de 5 .

La exposición a campos eléctricos de baja frecuencia causa respuestas biológicas bien definidas a través de efectos de cargas eléctricas superficiales. La prevención de los efectos dolorosos de cargas eléctricas superficiales inducidas en el cuerpo por la exposición es efectuada a través de los niveles de referencia.

\section{Efectos crónicos.}

La literatura sobre los efectos crónicos de los campos de baja frecuencia se evaluó en detalle por equipos científicos. La agencia de la OMS para la investigación del cáncer, la IARC evaluó los campos magnéticos de baja frecuencia en el año 2002 y las clasificó en la categoría 2B, "posiblemente cancerígeno para los seres humanos". La justificación de esta clasificación fue el resultado de los hallazgos epidemiológicos sobre leucemia infantil.

La ICNIRP opina que la evidencia científica existente actualmente, de que la exposición prolongada a los campos magnéticos de baja frecuencia está causalmente relacionada con el aumento del riesgo de leucemia, son demasiado débiles para constituir un elemento de fundamentación de las directrices básicas sobre la exposición. En particular, si la relación no es causal, una reducción en la exposición no proporciona ningún beneficio para la salud.

\section{AI-5. Directrices para limitar la exposición a los CEM}

Las guías para la exposición ocupacional y la exposición del público en general son independientes.

En las directrices para exposición ocupacional se consideran a adultos expuestos, en sus lugares de trabajo, a campos magnéticos y eléctricos, variables en el tiempo, de $1 \mathrm{~Hz}$ a $10 \mathrm{MHz}$, por lo general en condiciones conocidas, y como resultado de la ejecución de tareas $\mathrm{y}$ funciones habituales.

En contraste, la población en general se refiere a personas de todas las edades y con un estado de salud variado, que puede aumentar la variabilidad de las susceptibilidades individuales. En muchos casos, los miembros del público en general no son conscientes de su exposición a los CEM. Estas consideraciones ponen de manifiesto la necesidad de adoptar limitaciones a la exposición más exigentes para el público en general que para los trabajadores cuando son expuestos en un entorno laboral. 


\section{AI-6. Limitaciones básicas y niveles de referencia}

Las limitaciones a la exposición que se basan en magnitudes físicas, directamente relacionadas con los efectos establecidos sobre la salud, se designan limitaciones básicas. Bajo estas directrices, la cantidad física utilizada para especificar la limitación básica a la exposición a los CEM es la intensidad del campo eléctrico interno $\left(\mathrm{E}_{\mathrm{i}}\right)$, ya que es el campo eléctrico el que afecta a las células nerviosas y otras células electrosensibles.

La intensidad del campo eléctrico interno es difícil de evaluar. Así que a efectos prácticos de evaluación de la exposición, se proporcionan niveles de referencia para la exposición.

Los riesgos derivan de la respuesta transitoria del sistema nervioso, incluyendo la estimulación del sistema nervioso periférico (PNS) y el sistema nervioso central (CNS), la inducción de fosfenos sobre la retina y los posibles efectos sobre la actividad cerebral.

\section{AI-7. Niveles de referencia de las principales guías a nivel internacional}

Los valores límites recomendados por ICNIRP en 1998 [ 4 ], consideran los efectos de corto término, para lo cual establece que no se debe superar la densidad de corriente inducida en el cuerpo. Para la frecuencia de $50 \mathrm{~Hz}$, establece que la densidad de corriente para el público en general es de $2 \mathrm{~mA} / \mathrm{m}^{2}$, mientras que para ocupacional es de $10 \mathrm{~mA} / \mathrm{m}^{2}$. De estos límites surgen los valores de campo eléctrico y magnético para la población son $5 \mathrm{kV} / \mathrm{m}$ y $100 \mu \mathrm{T}$, respectivamente, mientras que a nivel ocupacional son de $10 \mathrm{kV} / \mathrm{m}$ y $500 \mu \mathrm{T}$.

En el año 2010, ICNIRP publica la Guía para los límites de exposición de campos magnéticos variables con el tiempo, con frecuencias en el rango de $1 \mathrm{~Hz}$ a $100 \mathrm{kHz}$ [ 3 ]. Los principales cambios, con respecto a la recomendación previa, se describen a continuación:

- La restricción básica considera el campo eléctrico interno inducido, en lugar de la densidad de corriente inducida en el cuerpo, ya que es la magnitud física que determina los efectos biológicos.

- Además se consideran en esta guía, los efectos en la retina (fosfenos).

En la Fig. AI 1 se muestran las restricciones básicas del campo eléctrico interno para exposición pública y ocupacional, considerando el sistema nervioso periférico (PNS) y el sistema nervioso central (CNS). Para la frecuencia de $50 \mathrm{~Hz}$ el límite de campo eléctrico interno inducido para el público en general es de $0,02 \mathrm{~V} / \mathrm{m}$, mientras que para ocupacional es de $0,1 \mathrm{~V} / \mathrm{m}$. Estos límites definen que los valores límites de exposición de campo magnético para la población es $200 \mu \mathrm{T}$ y a nivel ocupacional es de $1.000 \mu \mathrm{T}$, mientras que los valores límites de campo eléctrico para $50 \mathrm{~Hz}$ es de $5 \mathrm{kV} / \mathrm{m}$ para público en general y $10 \mathrm{kV} / \mathrm{m}$ para ocupacional. 


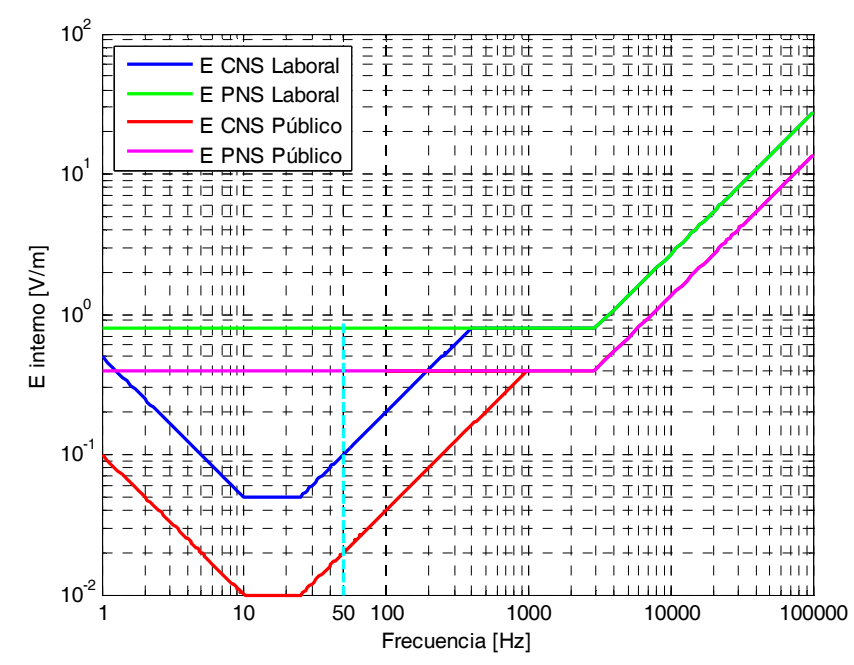

Fig. AI 1 - Restricciones básicas para el campo eléctrico interno (ICNIRP 2010)

Por otro lado el IEEE también realiza recomendaciones sobre los niveles de exposición a través de la Norma IEEE C95.6-2002 [ 13 ], las frecuencias allí consideradas van desde 0 a $3 \mathrm{kHz}$. La norma considera restricciones básicas para determinadas zonas del cuerpo en términos del campo eléctrico en el medio biológico. Para lo cual se considera el valor máximo permitido de campo eléctrico inducido in situ. Las restricciones básicas en el campo eléctrico in situ se aplican a un promedio aritmético determinado a lo largo de un segmento de línea recta de longitud de $0,5 \mathrm{~cm}$ orientado en cualquier dirección dentro de los tejidos del cerebro, corazón, manos, muñecas, pies y otros tejidos.

Esta norma considera la exposición de diferentes partes del cuerpo como ser cabeza y torso o brazos y piernas. Aquí se consideran como límites de exposición los correspondientes a cabeza y torso.

Debe considerarse que las referencias antes mencionadas abarcan un amplio espectro de frecuencias, pero aquí interesan las que corresponden a FEB.

En la Fig. AI 2 se realiza una comparación para el caso de exposición de público en general para las FEB, para el caso de IEEE se consideraron los límites de cabeza y torso. Se destaca que para el caso de frecuencia industrial $(50 \mathrm{~Hz})$ el límite recomendado por ICNIRP se ha incrementado de 100 a $200 \mu \mathrm{T}$ para el público en general y para IEEE el límite correspondiente es de $904 \mu \mathrm{T}$. El incremento llevado a cabo por ICNIRP se realiza ya que no se ha podido encontrar evidencias científicas de que a las frecuencias mencionadas se encuentren efectos para la salud. 


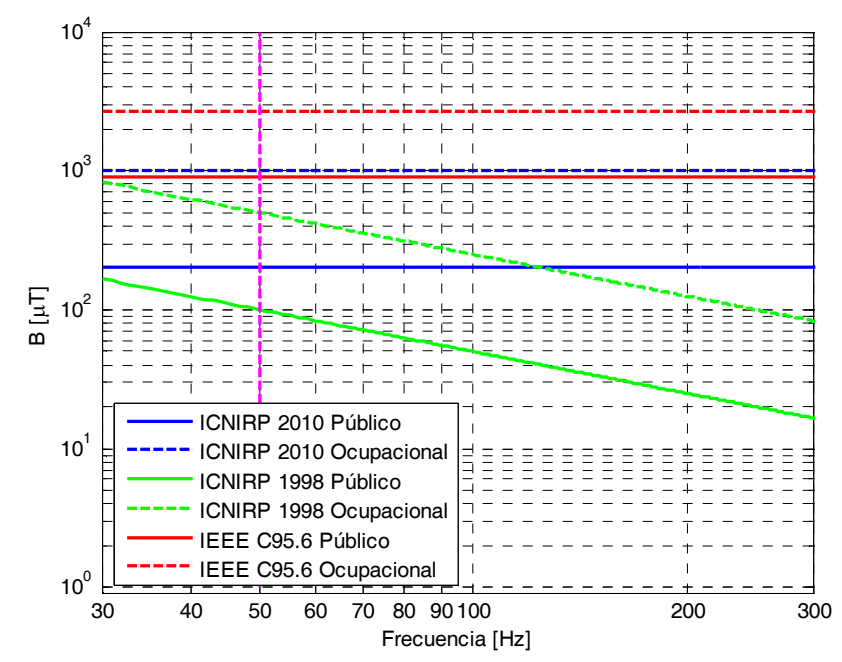

Fig. AI 2 - Niveles de referencia para exposición a campo magnético variable en el tiempo

En la Fig. AI 3 se aprecian los límites correspondientes al campo eléctrico en el rango de las FEB, para este caso ICNIRP no realizo cambios en los valores recomendados en 2010 con respecto a los valores de indicados en 1998.

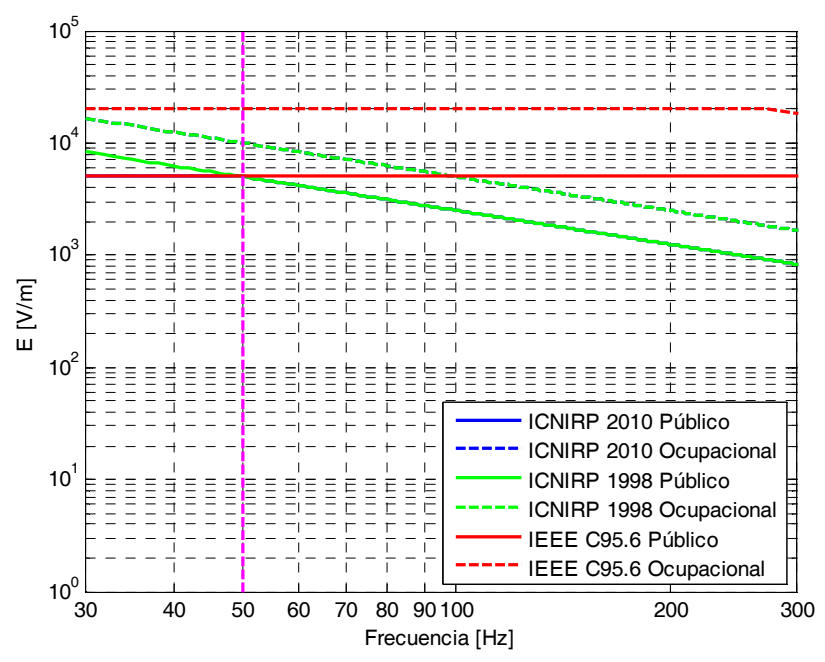

Fig. AI 3 - Niveles de referencia para exposición a campo eléctrico variable en el tiempo. Los límites recomendados por ICNIRP en 1998 y 2010 coinciden. 


\section{Anexo II}

Ecuaciones de Maxwell

Campo Magnético generado por un conductor rectilíneo e infinito

Campo Magnético debido a una Línea

Campo Magnético generado por una línea trifásica

Resistencia y Reactancia inductiva de circuitos con retorno por el suelo

Influencia de la tierra en el Campo Magnético generado por una Línea Aérea

Campo Magnético generado por una línea de transmisión con corrientes equilibradas

Campo Magnético generado por una línea de transmisión en general

Campo Magnético generado por un sistema de " $n$ " conductores paralelos 


\section{AII-1. Ecuaciones de Maxwell}

Los fenómenos electromagnéticos son descriptos por las ecuaciones de Maxwell, estas representan las propiedades generales de los campos electromagnéticos. A continuación se transcriben estas ecuaciones:

$$
\begin{aligned}
& \operatorname{div} B=0 \\
& \operatorname{div} D=\rho_{v} \\
& \operatorname{rot} H=J+\frac{\partial D}{\partial t} \\
& \operatorname{rot} E=-\frac{\partial B}{\partial t}
\end{aligned}
$$

La Ec. AII 1 describe la naturaleza del campo magnético, ella expresa el hecho de la inexistencia de los monopolos magnéticos,, en otros términos el flujo magnético siempre describe trayectorias cerradas.

La Ec. AII 2 describe la naturaleza del campo eléctrico, el término del lado derecho fundamenta la existencia de cargas eléctricas aisladas.

La Ec. AII 3 (Ley de Ampere) contiene los fundamentos de la generación de campos magnéticos debido a fenómenos eléctricos, el primer término del lado derecho representa las fuentes de corriente de conducción y el segundo la corriente de desplazamiento.

La Ec. AII 4 justifica la generación de campo eléctrico debido a fenómenos magnéticos, esto es la variación del flujo de campo magnético a través de una superficie induce un campo eléctrico que puede generar la circulación de una corriente a través de un lazo conductor que recorre el contorno de la mencionada superficie. El signo "-" representa (Ley de Lenz) el hecho de que la corriente inducida generará un campo que se opone a la causa que lo produce.

La Ley de Amper está dada por la Ec. AII 5

$\oint H . d l=I$

El campo magnético generado por un conductor cilíndrico esta dado por las Ec. AII 6 o Ec. AII 7.

$$
\begin{aligned}
& B_{r i}=\mu \frac{I_{i}}{2 \pi r_{i}}\left[\frac{W b}{m^{2}}\right] \\
& H_{r i}=\frac{I_{i}}{2 \pi r_{i}}\left[\frac{A}{m}\right]
\end{aligned}
$$

Donde:

$\mathrm{I}_{\mathrm{i}}$ : es la corriente por el conductor

$r_{i}$ : es la distancia medida desde el centro del conductor

$\mathrm{B}_{\mathrm{ri}}$ : es la densidad de campo magnético $[\mathrm{T}=\mathrm{Wb} / \mathrm{m} 2]$ 
En la bibliografía es común encontrar que se hace referencia a B y $\mathrm{H}$ indistintamente y se los identifica como "Campo Magnético". La relación existente entre B y H se describe en la Ec. AII 8.

$B=\mu H=\mu_{r} \mu_{0} H$

Ec. AII 8

A excepción de los materiales magnéticos, en general la permeabilidad un medio es igual a la de vacío o la del aire $\left(\mu_{\mathrm{r}}=1\right.$, entonces $\left.\mu_{=} \mu_{0}\right)$ y son aplicables las siguientes equivalencias:

$0,8[\mathrm{~A} / \mathrm{m}] \sim 1[\mu \mathrm{T}] \sim 10[\mathrm{mG}]$

\section{AII-2. Campo Magnético generado por un conductor rectilíneo e infinito}

Si ahora se considera un conductor largo, como los de las líneas de transmisión de energía, es posible utilizar la expresión obtenida anteriormente, si se considera que el campo magnético es una magnitud vectorial, se deben calcular las componentes del mismo, como se indica a continuación [ 26]:

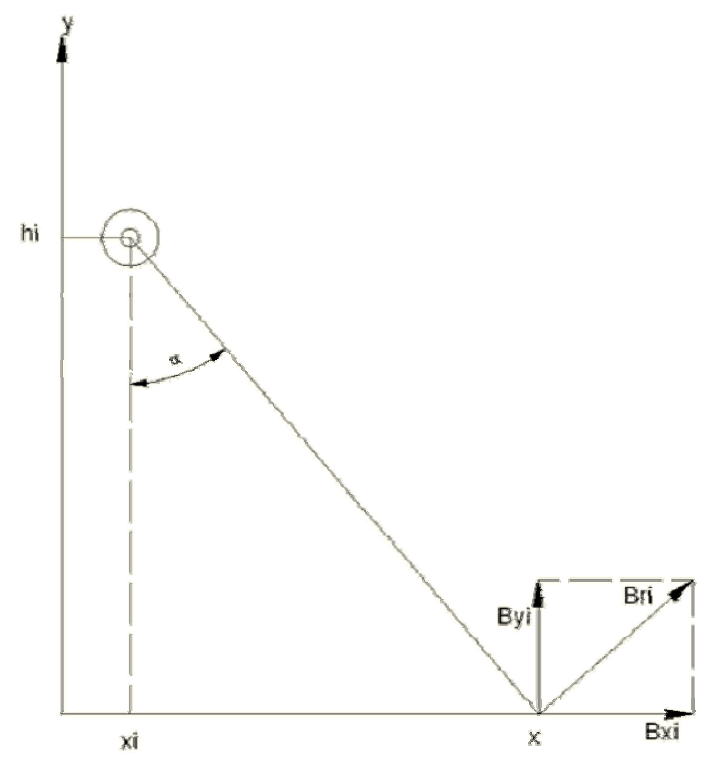

Fig. AII 1 - Conductor rectilíneo e infinito

$$
\begin{aligned}
& B_{x i}=B_{r i} \cos \left(\alpha_{i}\right)=\frac{\mu_{0} I_{i}}{\left.2 \pi \sqrt{\left[\left(x-x_{i}\right)^{2}+\left(y-h_{i}\right)^{2}\right.}\right]} \frac{\left(h_{i}-y\right)}{\left.\sqrt{\left[\left(x-x_{i}\right)^{2}+\left(y-h_{i}\right)^{2}\right.}\right]} \\
& B_{y i}=B_{r i} \operatorname{sen}\left(\alpha_{i}\right)=\frac{\mu_{0} I_{i}}{\left.2 \pi \sqrt{\left[\left(x-x_{i}\right)^{2}+\left(y-h_{i}\right)^{2}\right.}\right]} \sqrt{\left.\sqrt{\left(x-x_{i}\right)^{2}+\left(y-h_{i}\right)^{2}}\right]} \\
& B_{x i}=B_{r i} \cos \left(\alpha_{i}\right)=\frac{\mu_{0} I_{i}\left(h_{i}-y\right)}{2 \pi\left[\left(x-x_{i}\right)^{2}+\left(y-h_{i}\right)^{2}\right]} \\
& B_{y i}=B_{r i} \operatorname{sen}\left(\alpha_{i}\right)=\frac{\mu_{0} I_{i}\left(x-x_{i}\right)}{2 \pi\left[\left(x-x_{i}\right)^{2}+\left(y-h_{i}\right)^{2}\right]}
\end{aligned}
$$




\section{AII-3. Campo Magnético debido a una Línea}

Para calcular el campo magnético que generan las líneas de transmisión deben utilizarse las ecuaciones de Maxwell. Para el caso de las líneas de transmisión de energía eléctrica, la frecuencia en la que trabajan es extremadamente baja, esto permite simplificar las ecuaciones de Maxwell de forma tal que los cálculos sean realizados aplicando la Ley de BiotSavart.

En esta sección se presenta un procedimiento que permite conocer como se realizan los cálculos del campo magnético generado por líneas de transmisión de energía eléctrica. La ley de Biot-Savart considera a los conductores como filamentos de sección nula, esto es posible cuando el radio de los conductores es despreciable con respecto a la distancia de cálculo. Para los casos que se analizan el interés está centrado en distancias a $1 \mathrm{~m}$ sobre el nivel del suelo o en algunos casos sobre el nivel del suelo.

En primer lugar se considera un conductor rectilíneo e infinito, en general para las líneas posible realizar esta simplificación.

\section{AII-4. Campo Magnético generado por una línea trifásica}

Si ahora se considera una línea de transmisión de energía eléctrica, que se encuentra conformada por varios conductores, debe considerarse el aporte de cada uno de ellos Fig. AII 2.

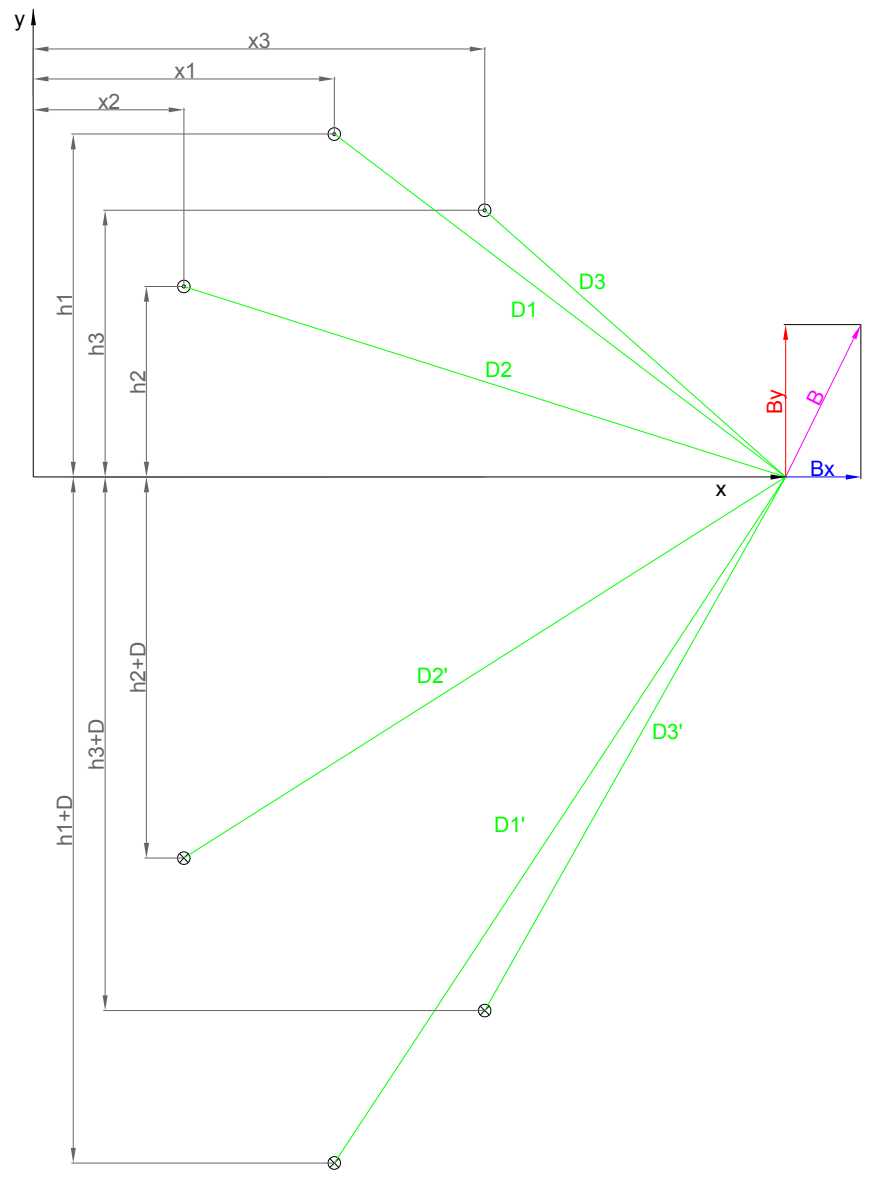

Fig. AII 2 - Campo magnético generado por una línea eléctrica

El campo total considerando las dos componentes por separado sobre el terreno estará dado por las ecuaciones Ec. AII 13 y Ec. AII 14. 


$$
\begin{aligned}
& \sum_{i=1}^{n} B_{x i}=\sum_{i=1}^{n}\left(\frac{\mu_{0} I_{i} h_{i}}{2 \pi\left[\left(x-x_{i}\right)^{2}+\left(h_{i}\right)^{2}\right]}+\frac{\mu_{0} I_{i}\left(h_{i}-D\right)}{2 \pi\left[\left(x-x_{i}\right)^{2}+\left(h_{i}+D\right)^{2}\right]}\right) \\
& \sum_{i=1}^{n} B_{y i}=\sum_{i=1}^{n}\left(\frac{\mu_{0} I_{i}\left(x-x_{i}\right)}{2 \pi\left[\left(x-x_{i}\right)^{2}+\left(h_{i}\right)^{2}\right]}+\frac{\mu_{0} I_{i}\left(x-x_{i}\right)}{2 \pi\left[\left(x-x_{i}\right)^{2}+\left(h_{i}+D\right)^{2}\right]}\right)
\end{aligned}
$$

\section{AII-5. Resistencia y Reactancia inductiva de circuitos con retorno por el suelo}

La presencia de un conductor, por el que circula corriente, sobre un medio también conductor, provoca que en el interior del medio se induzcan corrientes. Las líneas aéreas de transmisión de energía eléctrica se ubican sobre la tierra a una altura, que puede variar de acuerdo al tipo de línea [ 27 ].

Este tema fue abordado por Carson, quien consideró que los conductores de las líneas se disponen paralelos al terreno, la tierra posee resistividad uniforme y una extensión infinita. Carson demostró que las impedancias propias y mutuas de circuitos con retorno por la tierra, son iguales a las impedancias para un circuito en el que se considera un conductor imagen a la misma profundidad que la altura del conductor sobre el suelo, corregidas por un factor.

\section{AII-6. Método Exacto de Carson}

Carson consideró conductores paralelos al suelo, con resistividad uniforme y extensión infinita. Demostró que las impedancias propias y mutuas de circuitos con retorno por el suelo son iguales a las impedancias para un circuito sobre el terreno perfecto, en el que es posible considerar un conductor imagen a una profundidad igual a la altura del conductor sobre el suelo, pero afectada por un factor de corrección " $P+j Q$ ". Este factor depende de dos variables " $p$ " $\mathrm{y}$ " $\theta$ ", que serán definidas.

La función " $P+j Q$ " es común para las impedancias propias y mutuas, pero las variables, $p$ y $\theta$, son diferentes para las dos.

Se considera el circuito de la Fig. AII 3, en la que dos conductores a y b, cuyas alturas sobre el terreno son $h_{a} y h_{b}$ respectivamente, y las distancias a las imágenes $D_{a b} y D_{b a}$ respectivamente. 


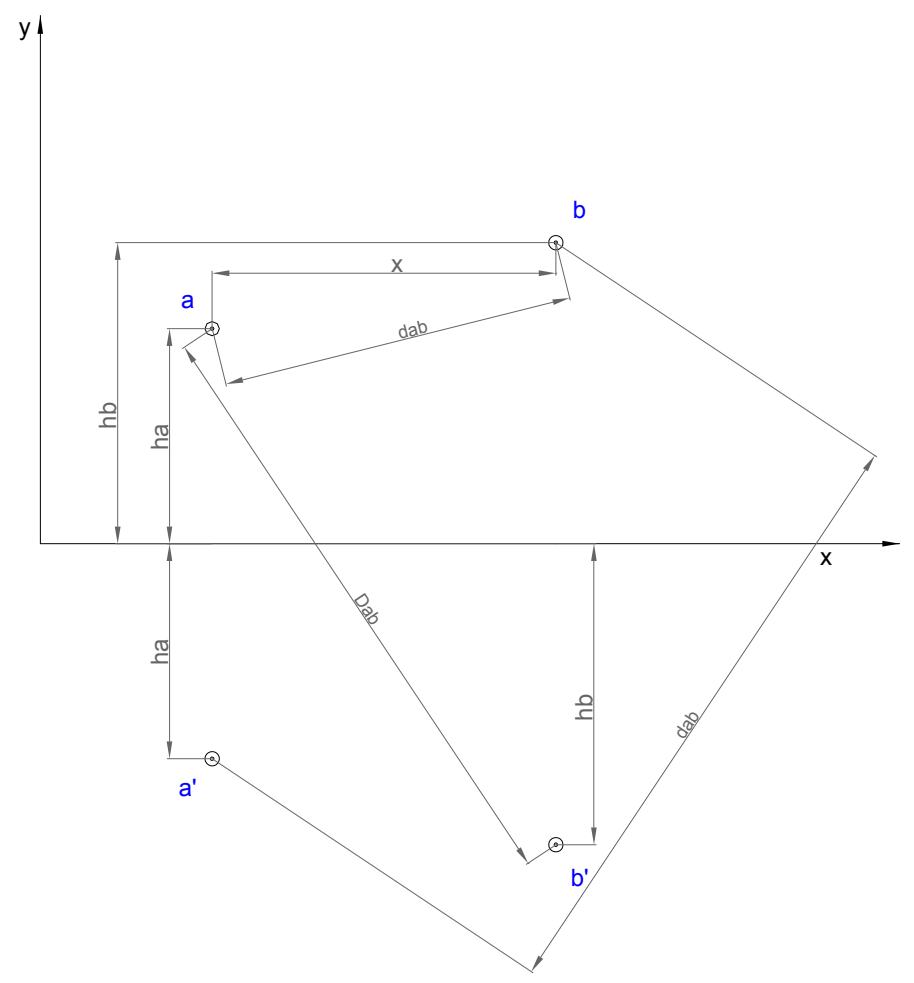

Fig. AII 3 - Conductores con retorno por el suelo

Se define la impedancia propia $\left(\mathrm{z}_{\mathrm{p}}\right)$ de los circuitos con retorno por el suelo, Ec. AII 15.

$$
z_{p}=r_{0}+j 28,935 \times 10^{-4} f\left(\log \frac{2 h_{l}}{D_{a L}}\right)+25,134 \times 10^{-4} f(P+j Q)\left[\frac{\Omega}{k m}\right]
$$

Se define la impedancia mutua $\left(\mathrm{z}_{\mathrm{M}}\right)$ de los circuitos con retorno por el suelo Ec. AII 16:

$$
z_{M}=28,935 \times 10^{-4} f\left(\log \frac{D_{a b}}{d_{a b}}\right)+25,134 \times 10^{-4} f(P+j Q)\left[\frac{\Omega}{k m}\right]
$$

Las variables $p$ y $\theta$, se determinan de la siguiente manera:

Para impedancias propias, $\theta=0$ y $p_{i}$ se determina por medio de la Ec. AII 17.

$$
p_{i}=5,620 \times 10^{-2} h_{i} \sqrt{\frac{f}{\rho}}
$$

$\rho$ : resistividad del suelo $\left[\Omega / \mathrm{m}^{3}\right]$

Para impedancias mutuas se aplican las Ec. AII 18 y Ec. AII 19.

$$
\begin{aligned}
& \theta_{i j}=\operatorname{tg}^{-1}\left(-\frac{x_{i j}}{h_{i}+h_{j}}\right) \\
& p_{i j}=28,1004 \times 10^{-4} D_{i j} \sqrt{\frac{f}{\rho}}
\end{aligned}
$$

Las ecuaciones para la determinación de $\mathrm{P}$ y $\mathrm{Q}$ se presentan Ec. AII 20 y Ec. AII 21 respectivamente. 


$$
\begin{aligned}
& P=\left[\frac{\pi}{8}-\frac{p}{3 \sqrt{2}} \cos \theta+\frac{p^{2}}{16} \cos 2 \theta\left(0,6728+\ln \frac{2}{p}\right)+\frac{p^{2}}{16} \theta \sin 2 \theta\right]\left[\frac{\Omega}{k m}\right] \\
& Q=\left[-0,0386-\frac{1}{2} \ln \frac{2}{p}+\frac{1}{3 \sqrt{2}} p \cos \theta\right]\left[\frac{\Omega}{\mathrm{km}}\right]
\end{aligned}
$$

Con los valores de $\mathrm{P}$ y $\mathrm{Q}$, se obtienen las matrices de corrección de las matrices de resistencias y reactancias inductivas, cuyo orden es igual al número de conductores, Ec. AII 22.

$\left[Z_{c o r r}\right]=\left[\begin{array}{ccc}r_{a a} & \ldots & 0 \\ \vdots & \ddots & \vdots \\ 0 & \cdots & r_{s s}\end{array}\right]+k\left[\begin{array}{ccc}P_{a a} & \ldots & P_{a s} \\ \vdots & \ddots & \vdots \\ P_{s a} & \cdots & P_{s s}\end{array}\right]+j\left\{\left[\begin{array}{ccc}x_{a a} & \ldots & x_{a s} \\ \vdots & \ddots & \vdots \\ x_{s a} & \cdots & x_{s s}\end{array}\right]+k\left[\begin{array}{ccc}Q_{a a} & \ldots & Q_{a s} \\ \vdots & \ddots & \vdots \\ Q_{s a} & \cdots & Q_{s s}\end{array}\right]\right\}$

$k=25,134 \times 10^{-4} f$

Ec. AII 23

La matriz de impedancia resultante puede ser reducida a una matriz de $3 \mathrm{x} 3$ y en sus términos estarán considerados los factores de corrección que consideren las características del terreno y la presencia de cables de guarda.

\section{AII-7. Método Aproximado}

Existen aproximaciones que permiten simplificar el problema e introducen errores admisibles. Estas consisten en despreciar los términos que contienen a " $\theta$ ". En estas condiciones el factor de corrección de la resistencia de la tierra es constante y proporcional a la frecuencia de operación del sistema. El término de corrección para la reactancia inductiva resulta proporcional a la resistividad del suelo e inversamente proporcional a la frecuencia.

En estas condiciones las impedancias propias $\left(\mathrm{z}_{\mathrm{i}}\right)$ y mutuas $\left(\mathrm{z}_{\mathrm{ij}}\right)$ estan dadas por las Ec. AII 24 y Ec. AII 25, respectivamente.

$$
\begin{aligned}
& z_{i}=r_{0 i}+9,88 \times 10^{-4} f+j 28,935325 \times 10^{-4} f\left(\log \frac{658,368 \sqrt{\frac{\rho}{f}}}{D_{0 i}}\right)\left[\frac{\Omega}{k m}\right] \\
& z_{i j}=9,88 \times 10^{-4} f+j 28,935325 \times 10^{-4} f\left(\log \frac{658,368 \sqrt{\frac{\rho}{f}}}{d_{i j}}\right]_{\left[\frac{\Omega}{k m}\right]}
\end{aligned}
$$

Definiendo la distancia “D” por medio de la Ec. AII 26.

$$
D=658,368 \sqrt{\frac{\rho}{f}} \cong 658,4 \sqrt{\frac{\rho}{f}}
$$

La distancia D, puede ser interpretada como la distancia entre los conductores de fase y un único conductor de diámetro unitario, que sirve de retorno para los conductores de la línea, debido que los valores de D resultan muy grandes comparados con las distancias entre conductores de fase. 


\section{AII-8. Influencia de la tierra en el Campo Magnético generado por una Línea Aérea}

La presencia de un conductor, por el que circula corriente, sobre un medio también conductor, provoca que en el interior del medio se induzcan corrientes. Las líneas aéreas de transmisión de energía eléctrica se ubican a sobre la tierra a una altura, que puede variar de acuerdo al tipo de línea [ 27 ]. En el Inciso A II - 5 se analizó la influencia del terreno.

Si se considera la Fig. AII 2, es posible calcular la distancia "D" por medio de la Ec. AII 26. En la Tabla AII 1 se presentan resistividades típicas de suelos y las distancias "D" correspondientes, considerando una frecuencia de $50 \mathrm{~Hz}$.

Tabla AII 1 - Resistividades típicas de suelos y distancias D, para $50 \mathrm{~Hz}$.

\begin{tabular}{|c|c|c|}
\hline Terreno & $\mathbf{P}[\mathbf{\Omega m}]$ & $\mathbf{D}$ \\
\hline Agua de mar & $0,01-1$ & $9,3-93,1$ \\
\hline Terreno pantanoso & $10-100$ & $294,4-931$ \\
\hline Tierra seca & 1000 & 2944 \\
\hline Pedregullo & 10000000 & 294445 \\
\hline Arena & 1000000000 & 2944454 \\
\hline Valor medio & 100 & 931 \\
\hline
\end{tabular}

Para $50 \mathrm{~Hz}$ y terrenos cuya resistividad media es de $100 \Omega \mathrm{m}(\mathrm{D}=931 \mathrm{~m})$, la profundidad del conductor imagen es mucho mayor que la altura del conductor real sobre el terreno (para LAAT de $500 \mathrm{kV}$ la altura media puede ser de alrededor de $25 \mathrm{~m}$ ), se puede decir que la influencia del conductor imagen es despreciable si se pretende medir o calcular el campo magnético a un metro sobre el nivel del suelo.

\section{AII-9. Campo Magnético generado por una línea de transmisión con corrientes equilibradas}

Si se considera un sistema trifásico de corrientes equilibradas, es posible expresar las dos componentes en función de las corrientes.

$$
\begin{aligned}
& B_{x}=\frac{\mu_{0} h_{1} I \operatorname{sen}(\omega t)}{2 \pi\left[\left(x-x_{1}\right)^{2}+\left(h_{1}\right)^{2}\right]}+\frac{\mu_{0} h_{2} I \operatorname{sen}(\omega t-120)}{2 \pi\left[\left(x-x_{2}\right)^{2}+\left(h_{2}\right)^{2}\right]}+\frac{\mu_{0} h_{3} I \operatorname{sen}(\omega t-240)}{2 \pi\left[\left(x-x_{3}\right)^{2}+\left(h_{3}\right)^{2}\right]} \\
& B_{y}=\frac{\mu_{0}\left(x-x_{1}\right) I \operatorname{sen}(\omega t)}{2 \pi\left[\left(x-x_{1}\right)^{2}+\left(h_{1}\right)^{2}\right]}+\frac{\mu_{0}\left(x-x_{2}\right) I \operatorname{sen}(\omega t-120)}{2 \pi\left[\left(x-x_{2}\right)^{2}+\left(h_{2}\right)^{2}\right]}+\frac{\mu_{0}\left(x-x_{3}\right) I \operatorname{sen}(\omega t-120)}{2 \pi\left[\left(x-x_{3}\right)^{2}+\left(h_{3}\right)^{2}\right]}
\end{aligned}
$$

Considerando las siguientes igualdades:
$\mathrm{D}_{1}^{2}=\left(\mathrm{x}-\mathrm{x}_{1}\right)^{2}+\mathrm{h}_{1}^{2}$
$\cos (120)=\cos (240)=-\frac{1}{2}$
$\mathrm{D}_{2}{ }^{2}=\left(\mathrm{x}-\mathrm{x}_{2}\right)^{2}+\mathrm{h}_{2}{ }^{2}$
$\operatorname{sen}(120)=\frac{\sqrt{3}}{2}$
$\mathrm{D}_{3}{ }^{2}=\left(\mathrm{x}-\mathrm{x}_{3}\right)^{2}+\mathrm{h}_{3}{ }^{2}$
$\operatorname{sen}(240)=-\frac{\sqrt{3}}{2}$ 
Es posible expresar las Ec. AII 27 y Ec. AII 28 por medio de las Ec. AII 29 y Ec. AII 30, respectivamente.

$$
\begin{aligned}
B_{x}= & \frac{\mu_{0} I}{2 \pi}\left[\frac{h_{1}}{D_{1}{ }^{2}}-\frac{h_{2}}{2 D_{2}{ }^{2}}-\frac{h_{3}}{2 D_{3}{ }^{2}}\right] \operatorname{sen}(\omega t)+ \\
& +\frac{\mu_{0} I}{2 \pi}\left[\frac{\sqrt{3} h_{3}}{2 D_{3}{ }^{2}}-\frac{\sqrt{3} h_{2}}{2 D_{2}{ }^{2}}\right] \cos (\omega t) \\
B_{y}= & \frac{\mu_{0} I}{2 \pi}\left[\frac{\left(x-x_{1}\right)}{D_{1}{ }^{2}}-\frac{\left(x-x_{2}\right)}{2 D_{2}{ }^{2}}-\frac{\left(x-x_{3}\right)}{2 D_{3}{ }^{2}}\right] \operatorname{sen}(\omega t)+ \\
& +\frac{\mu_{0} I}{2 \pi}\left[\frac{\sqrt{3}\left(x-x_{3}\right)}{2 D_{3}{ }^{2}}-\frac{\sqrt{3}\left(x-x_{2}\right)}{2 D_{2}{ }^{2}}\right] \cos (\omega t)
\end{aligned}
$$

\section{AII-10. Campo Magnético generado por una línea de transmisión en general}

En general las corrientes de una línea de transmisión no se encuentran necesariamente en fase, ya que dependen de la carga y el ángulo de fase de la tensión. Por lo que resulta de interés conocer las expresiones de las componentes de campo magnético considerando una terna de corrientes en general.

Si se consideran las Ec. AII 27 y Ec. AII 28 y se les asigna a las corrientes ángulos de fase genéricos, $\Phi_{1}, \Phi_{2}$ y $\Phi_{3}$ es posible reescribirlas como las Ec. AII 31 y Ec. AII 32, respectivamente.

$$
\begin{aligned}
& B_{x}=\frac{\mu_{0} h_{1} I_{1} \operatorname{sen}\left(\omega t+\phi_{1}\right)}{2 \pi\left[\left(x-x_{1}\right)^{2}+\left(h_{1}\right)^{2}\right]}+\frac{\mu_{0} h_{2} I_{2} \operatorname{sen}\left(\omega t+\phi_{2}\right)}{2 \pi\left[\left(x-x_{2}\right)^{2}+\left(h_{2}\right)^{2}\right]}+\frac{\mu_{0} h_{3} I_{3} \operatorname{sen}\left(\omega t+\phi_{3}\right)}{2 \pi\left[\left(x-x_{3}\right)^{2}+\left(h_{3}\right)^{2}\right]} \\
& B_{y}=\frac{\mu_{0}\left(x-x_{1}\right) I_{1} \operatorname{sen}\left(\omega t+\phi_{1}\right)}{2 \pi\left[\left(x-x_{1}\right)^{2}+\left(h_{1}\right)^{2}\right]}+\frac{\mu_{0}\left(x-x_{2}\right) I_{2} \operatorname{sen}\left(\omega t+\phi_{2}\right)}{2 \pi\left[\left(x-x_{2}\right)^{2}+\left(h_{2}\right)^{2}\right]}+\frac{\mu_{0}\left(x-x_{3}\right) I_{3} \operatorname{sen}(\omega t+}{2 \pi\left[\left(x-x_{3}\right)^{2}+\left(h_{3}\right)^{2}\right.}
\end{aligned}
$$

Operando:

$$
\begin{aligned}
B_{x}= & \frac{\mu_{0}}{2 \pi}\left[\frac{I_{1} \cos \left(\phi_{1}\right) h_{1}}{D_{1}{ }^{2}}+\frac{I_{2} \cos \left(\phi_{2}\right) h_{2}}{D_{2}{ }^{2}}+\frac{I_{3} \cos \left(\phi_{3}\right) h_{3}}{D_{3}{ }^{2}}\right] \operatorname{sen}(\omega t)+ \\
& +\frac{\mu_{0}}{2 \pi}\left[\frac{I_{1} \operatorname{sen}\left(\phi_{1}\right) h_{1}}{D_{1}{ }^{2}}+\frac{I_{2} \operatorname{sen}\left(\phi_{2}\right) h_{2}}{D_{2}{ }^{2}}+\frac{I_{3} \operatorname{sen}\left(\phi_{3}\right) h_{3}}{D_{3}{ }^{2}}\right] \cos (\omega t) \\
B_{y}= & \frac{\mu_{0}}{2 \pi}\left[\frac{I_{1} \cos \left(\phi_{1}\right)\left(x-x_{1}\right)}{D_{1}{ }^{2}}+\frac{I_{2} \cos \left(\phi_{2}\right)\left(x-x_{2}\right)}{D_{2}{ }^{2}}+\frac{I_{3} \cos \left(\phi_{3}\right)\left(x-x_{3}\right)}{D_{3}{ }^{2}}\right] \operatorname{sen}(\omega t)+ \\
& +\frac{\mu_{0}}{2 \pi}\left[\frac{I_{1} \operatorname{sen}\left(\phi_{1}\right)\left(x-x_{1}\right)}{D_{1}{ }^{2}}+\frac{I_{2} \operatorname{sen}\left(\phi_{2}\right)\left(x-x_{2}\right)}{D_{2}{ }^{2}}+\frac{I_{3} \operatorname{sen}\left(\phi_{3}\right)\left(x-x_{3}\right)}{D_{3}{ }^{2}}\right] \cos (\omega t)
\end{aligned}
$$


A partir de las Ec. AII 33 y Ec. AII 34, es posible reescribir los términos que son independientes del tiempo, esto se presenta en las Ec. AII 35, Ec. AII 36, Ec. AII 37 y Ec. AII 38.

$$
\begin{aligned}
& A_{x}=\frac{\mu_{0}}{2 \pi}\left[\frac{I_{1} \cos \left(\varphi_{1}\right) h_{1}}{D_{1}{ }^{2}}+\frac{I_{2} \cos \left(\varphi_{2}\right) h_{2}}{D_{2}{ }^{2}}+\frac{I_{3} \cos \left(\varphi_{3}\right) h_{3}}{D_{3}{ }^{2}}\right] \\
& C_{x}=\frac{\mu_{0}}{2 \pi}\left[\frac{I_{1} \operatorname{sen}\left(\varphi_{1}\right) h_{1}}{D_{1}{ }^{2}}+\frac{I_{2} \operatorname{sen}\left(\varphi_{2}\right) h_{2}}{D_{2}{ }^{2}}+\frac{I_{3} \operatorname{sen}\left(\varphi_{3}\right) h_{3}}{D_{3}{ }^{2}}\right] \\
& A_{y}=\frac{\mu_{0}}{2 \pi}\left[\frac{I_{1} \cos \left(\varphi_{1}\right)\left(x-x_{1}\right)}{D_{1}{ }^{2}}+\frac{I_{2} \cos \left(\varphi_{2}\right)\left(x-x_{2}\right)}{D_{2}{ }^{2}}+\frac{I_{3} \cos \left(\varphi_{3}\right)\left(x-x_{3}\right)}{D_{3}{ }^{2}}\right] \\
& C_{y}=\frac{\mu_{0}}{2 \pi}\left[\frac{I_{1} \operatorname{sen}\left(\varphi_{1}\right)\left(x-x_{1}\right)}{D_{1}{ }^{2}}+\frac{I_{2} \operatorname{sen}\left(\varphi_{2}\right)\left(x-x_{2}\right)}{D_{2}{ }^{2}}+\frac{I_{3} \operatorname{sen}\left(\varphi_{3}\right)\left(x-x_{3}\right)}{D_{3}{ }^{2}}\right]
\end{aligned}
$$

Luego se pueden reescribir las Ec. AII 33 y Ec. AII 34 de la forma que se presentan en las Ec. AII 39 y Ec. AII 40, respectivamente.

$$
\begin{aligned}
& B_{x}(t)=A_{x} \operatorname{sen}(\omega t)+C_{x} \cos (\omega t) \\
& B_{y}(t)=A_{y} \operatorname{sen}(\omega t)+C_{y} \cos (\omega t)
\end{aligned}
$$

Considerando las relaciones presentadas en las Ec. AII 41 y Ec. AII 42, obtenidas a partir de las Ec. AII 39 y Ec. AII 40, es posible reescribir estas últimas de la manera presentada en las Ec. AII 43 y Ec. AII 44.

$$
\begin{aligned}
& \phi_{x}=\arctan \left(\frac{C_{x}}{A_{x}}\right) \\
& \phi_{y}=\arctan \left(\frac{C_{y}}{A_{y}}\right) \\
& B_{x}(t)=B_{x \max } \operatorname{sen}\left(\omega t+\phi_{x}\right) \\
& B_{y}(t)=B_{y \max } \operatorname{sen}\left(\omega t+\phi_{y}\right)
\end{aligned}
$$

Finalmente, es posible obtener el módulo del vector campo magnético en el espacio como se presenta en la Ec. AII 45.

$|B(t)|=\sqrt{\left(B_{x}(t)\right)^{2}+\left(B_{y}(t)\right)^{2}}$

Ec. AII 45 
AII-11. Campo Magnético generado por un sistema de " $\mathrm{n}$ " conductores paralelos

Si se tiene un sistema de "n" conductores como el de la Fig. AII 4, las componentes de campo magnético Bx y By, generadas por los "n" conductores están dados por las Ec. AII 46 y Ec. AII 47.

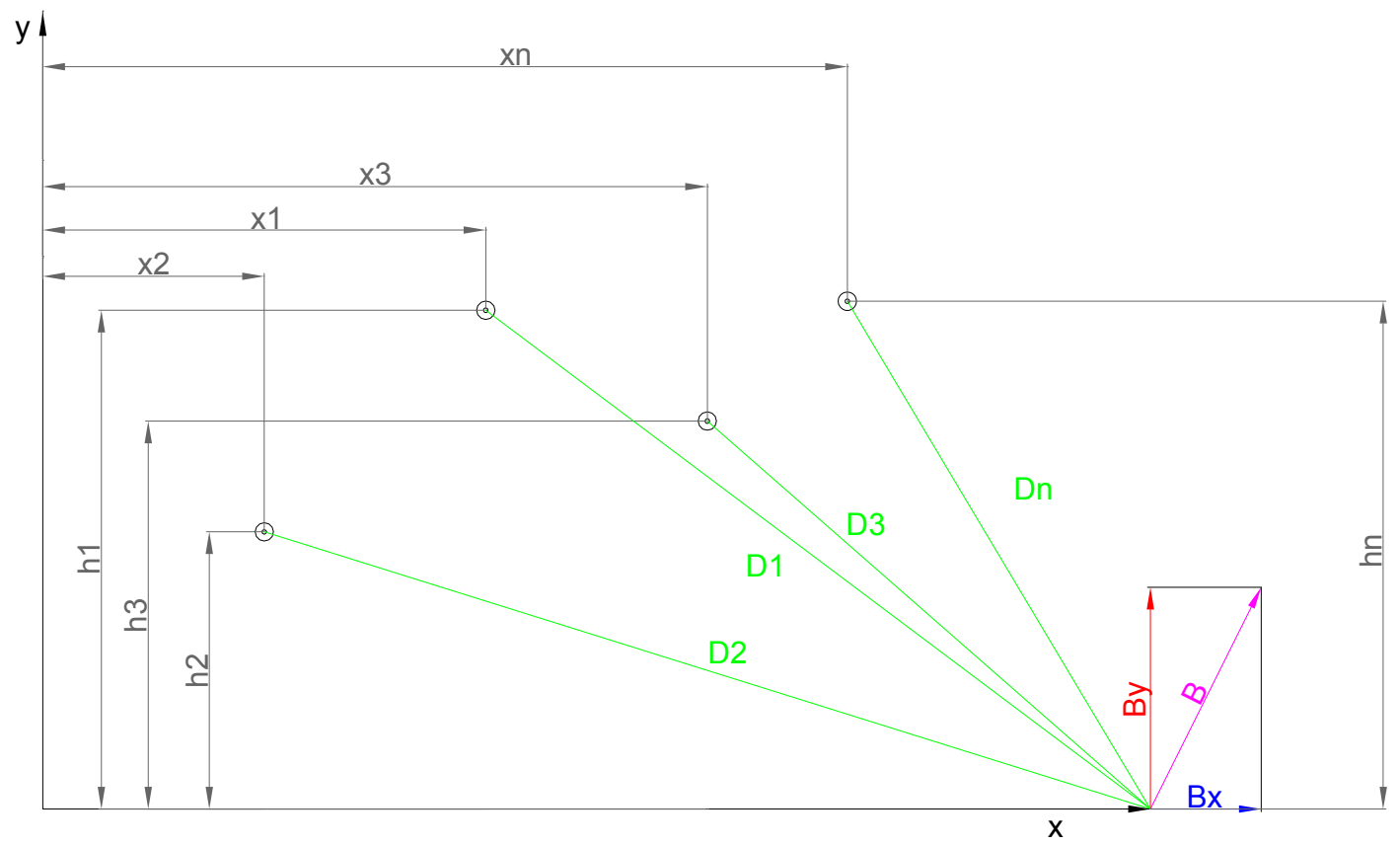

Fig. AII 4 - Campo Magnético generado por "n" conductores en paralelo

$$
\begin{aligned}
B_{x}= & \mu_{0} \pi\left[\begin{array}{l}
\frac{I_{1} \cos \left(\varphi_{1}\right) h_{1}}{D_{1}{ }^{2}}+\frac{I_{2} \cos \left(\varphi_{2}\right) h_{2}}{D_{2}{ }^{2}}+\frac{I_{3} \cos \left(\varphi_{3}\right) h_{3}}{D_{3}{ }^{2}}+\ldots \\
\ldots+\frac{I_{n} \cos \left(\varphi_{n}\right) h_{n}}{D_{n}{ }^{2}}
\end{array}\right] \operatorname{sen}(\omega t)+ \\
& +\frac{\mu_{0}}{2 \pi}\left[\begin{array}{l}
\frac{I_{1} \operatorname{sen}\left(\varphi_{1}\right) h_{1}}{D_{1}{ }^{2}}+\frac{I_{2} \operatorname{sen}\left(\varphi_{2}\right) h_{2}}{D_{2}{ }^{2}}+\frac{I_{3} \operatorname{sen}\left(\varphi_{3}\right) h_{3}}{D_{3}{ }^{2}}+\ldots \\
\ldots+\frac{I_{n} \operatorname{sen}\left(\varphi_{n}\right) h_{n}}{D_{n}{ }^{2}}
\end{array}\right] \cos (\omega t) \\
B_{y}= & \mu_{0} \pi\left[\begin{array}{l}
\frac{I_{1} \cos \left(\varphi_{1}\right)\left(x-x_{1}\right)}{D_{1}{ }^{2}}+\frac{I_{2} \cos \left(\varphi_{2}\right)\left(x-x_{2}\right)}{D_{2}{ }^{2}}+\frac{I_{3} \cos \left(\varphi_{3}\right)\left(x-x_{3}\right)}{D_{3}{ }^{2}}+\ldots \\
\ldots+\frac{I_{n} \cos \left(\varphi_{n}\right)\left(x-x_{n}\right)}{D_{n}{ }^{2}}
\end{array}\right] \operatorname{sen}(\omega t)+ \\
& +\frac{\mu_{0}}{2 \pi}\left[\begin{array}{l}
\frac{I_{1} \operatorname{sen}\left(\varphi_{1}\right)\left(x-x_{1}\right)}{D_{1}{ }^{2}}+\frac{I_{2} \operatorname{sen}\left(\varphi_{2}\right)\left(x-x_{2}\right)}{D_{2}{ }^{2}}+\frac{I_{3} \operatorname{sen}\left(\varphi_{3}\right)\left(x-x_{3}\right)}{D_{3}{ }^{2}}+\ldots \\
\ldots+\frac{I_{n} \operatorname{sen}\left(\varphi_{n}\right)\left(x-x_{n}\right)}{D_{n}{ }^{2}}
\end{array}\right] \cos (\omega t)
\end{aligned}
$$


En las Ec. AII 46 y Ec. AII 47 se observa que las componentes del campo magnético dependen de los valores de corrientes, en modulo y fase, y de la posición de los " $n$ " conductores. A partir de estas ecuaciones es posible expresar los términos que son independientes del tiempo, estos se realiza determinando los coeficientes que se identifican como "Axi, Ayi, Cxi y Cyi” y se calculan en forma general como se indica en las Ec. AII 48 a Ec. AII 51

$$
\begin{aligned}
A_{x i} & =\frac{\mu_{0}}{2 \pi} \frac{I_{i} \cos \left(\varphi_{i}\right) h_{i}}{D_{i}^{2}} \\
C_{x i} & =\frac{\mu_{0}}{2 \pi} \frac{I_{i} \operatorname{sen}\left(\varphi_{i}\right) h_{i}}{D_{i}^{2}} \\
A_{y i} & =\frac{\mu_{0}}{2 \pi} \frac{I_{i} \cos \left(\varphi_{i}\right)\left(x-x_{i}\right)}{D_{i}^{2}} \\
C_{y i} & =\frac{\mu_{0}}{2 \pi} \frac{I_{i} \operatorname{sen}\left(\varphi_{i}\right)\left(x-x_{i}\right)}{D_{i}{ }^{2}}
\end{aligned}
$$

Como se tiene un sistema de " $n$ " conductores, es necesario considerar el aporte de cada uno de ellos. Esto se realiza considerando las sumatorias se indicadas en las Ec. AII 52 a Ec. AII 55 .

$$
\begin{aligned}
& A_{x}=\sum_{i=1}^{n} A_{x i} \\
& C_{x}=\sum_{i=1}^{n} C_{x i} \\
& A_{y}=\sum_{i=1}^{n} A_{y i} \\
& C_{y}=\sum_{i=1}^{n} C_{y i}
\end{aligned}
$$

Ec. AII 55

Las componentes en los ejes "x e y" del campo magnético se determinan de acuerdo a lo expresado en Ec. AII 56 y Ec. AII 57

$$
\begin{aligned}
& B_{x}=\sqrt{A_{x}^{2}+C_{x}^{2}} \\
& B_{y}=\sqrt{A_{y}^{2}+C_{y}^{2}}
\end{aligned}
$$

El módulo del campo magnético $\left(B_{R}\right)$ queda definido a partir de las componentes como se indica en la Ec. AII 58.

$$
B_{R}=\sqrt{B_{x}^{2}+B_{y}^{2}}
$$




\section{Anexo III}

Polarización del campo magnético

Ejemplos de polarización del campo magnético

Datos geométricos de LAAT y CSAT 


\section{AIII-1. Polarización del campo magnético}

En general el campo magnético, generado por sistemas trifásicos de CA, en un punto del espacio puede representarse por un vector que describe el movimiento de una elipse en cada ciclo de la corriente por los conductores. Esto se presenta en la Fig. AIII 1. La magnitud y dirección del eje mayor de la elipse $\left(B_{\text {máx}}\right)$ indica la dirección y magnitud del campo magnético máximo. El eje menor $\left(\mathrm{B}_{\mathrm{min}}\right)$ indica la dirección y magnitud del campo magnético mínimo. A este tipo de campo se los identifica como elípticamente polarizado [11 ] [30].

El grado de polarización se define por la relación de los valores de densidades de flujo magnético entre el eje mayor y el eje menor.

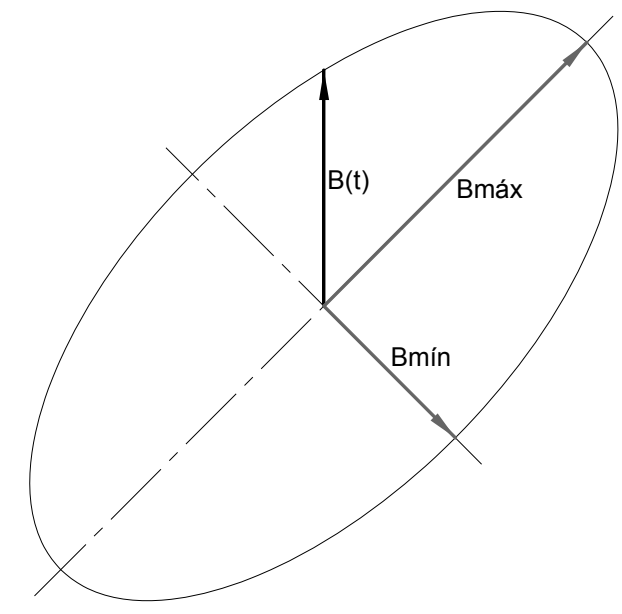

Fig. AIII 1 - Campo magnético polarizado elípticamente.

En zonas alejadas de las líneas de transmisión de energía, el campo magnético puede ser producido por diferentes fuentes, las que no necesariamente están en fase. Es posible encontrar entonces dos situaciones particulares. La polarización puede ser circular $\left(B_{\text {máx }}=B_{\text {mín }}\right)$ o lineal $\left(B_{\text {mín }}=0\right)$. Estas situaciones se aprecian en la Fig. AIII 2 y Fig. AIII 3, respectivamente.

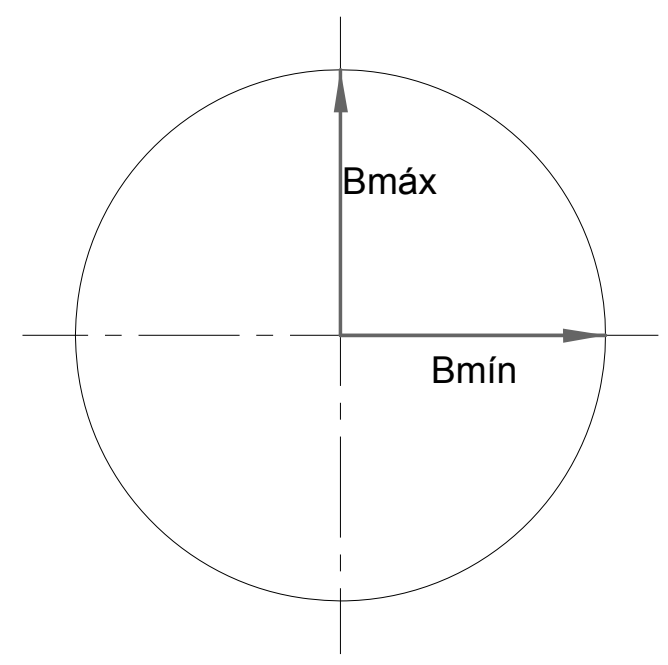

Fig. AIII 2 - Campo magnético con polarización circular. 


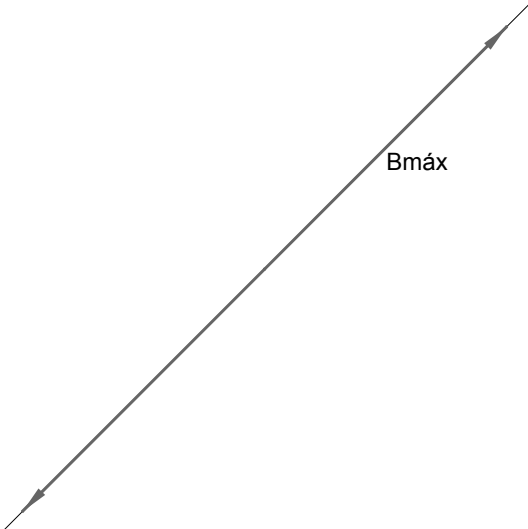

Fig. AIII 3 - Campo magnético con polarización lineal.

El campo magnético máximo y el campo magnético resultante coinciden únicamente en el caso de polarización lineal. La máxima diferencia entre el campo resultante y el campo magnético máximo se da en el caso de polarización circular, en el que el resultante puede llegar a ser $41 \%$ mayor que el Bmáx.

A partir de las Ec. AII 43 y Ec. AII 44 es posible escribir al campo total en función del tiempo, esto se presenta en la Ec. AIII 1.

$\vec{B}(t)=B_{x}(t) \overrightarrow{u_{x}}+B_{y}(t) \overrightarrow{u_{y}}=B_{x \max } \operatorname{sen}(\omega t) \overrightarrow{u_{x}}+B_{y \max } \operatorname{sen}\left(\omega t+\varphi_{x y}\right) \overrightarrow{u_{y}}$

Ec. AIII 1

Donde $\overline{\mathrm{u}}_{\mathrm{x}} \mathrm{y} \overline{\mathrm{u}}_{\mathrm{y}}$ son los vectores unitarios en los ejes $\mathrm{x}$ e $\mathrm{y}$.

En el caso de líneas aéreas de transmisión de energía o cables subterráneos, el plano en el que se encuentra la elipse es ortogonal a la dirección de los conductores. A estos se los conoce como sistemas cuasi 2D [11].

A continuación se presentan ejemplos de los tres tipos de polarización mencionados. En la Fig. AIII 4 se presenta el correspondiente a polarización elíptica, en a) se aprecia la variación temporal y en b) la espacial.

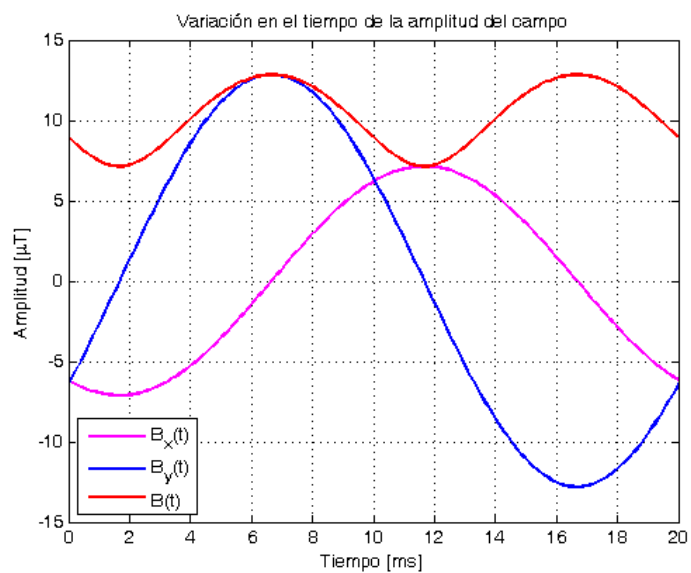

a)Variación temporal

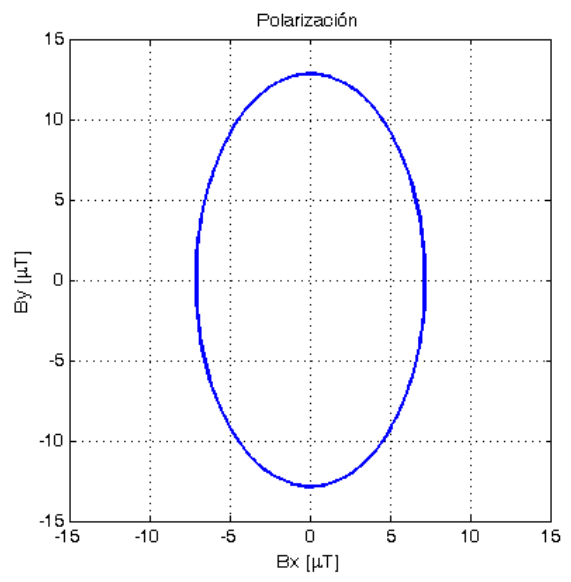

a)Variación espacial

Fig. AIII 4 - Ejemplo de polarización elíptica, $\mathrm{B}_{\mathrm{xmàx}} \neq \mathrm{B}_{\mathrm{xmàx}}$.

El la Fig. AIII 5 se presenta el correspondiente a polarización circular, en a) se aprecia la variación temporal y en b) la espacial. 


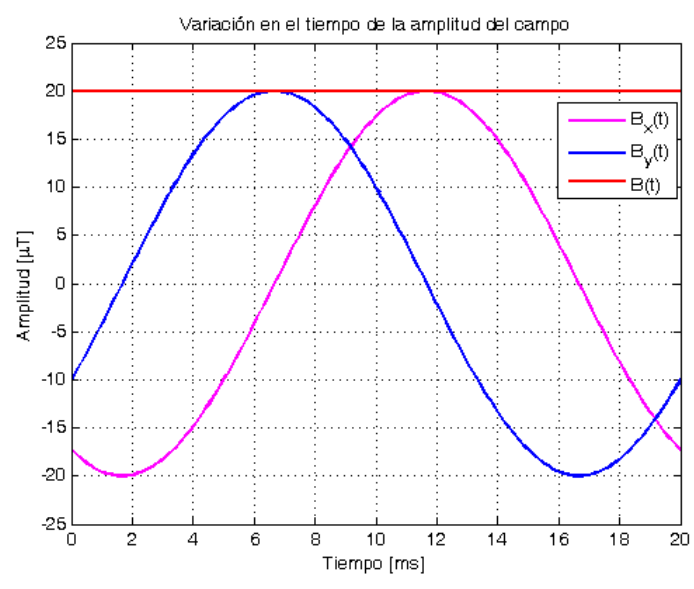

a)Variación temporal

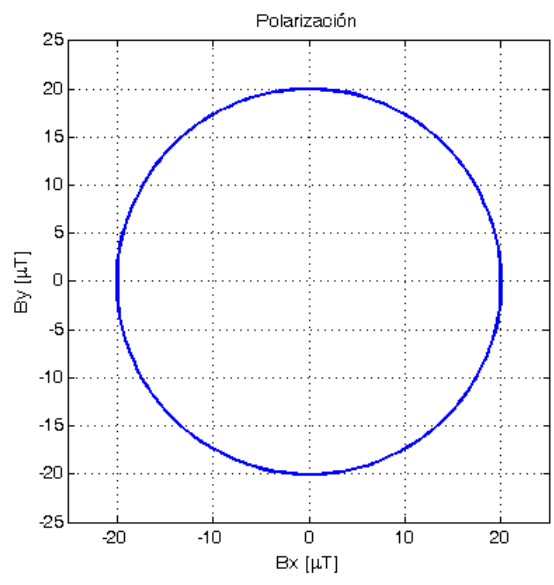

a)Variación espacial

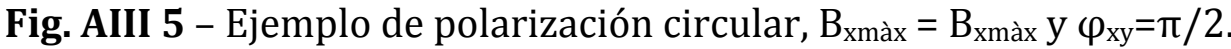

En la Fig. AIII 6 se presenta el correspondiente a polarización lineal, en a) se aprecia la variación temporal y en b) la espacial.

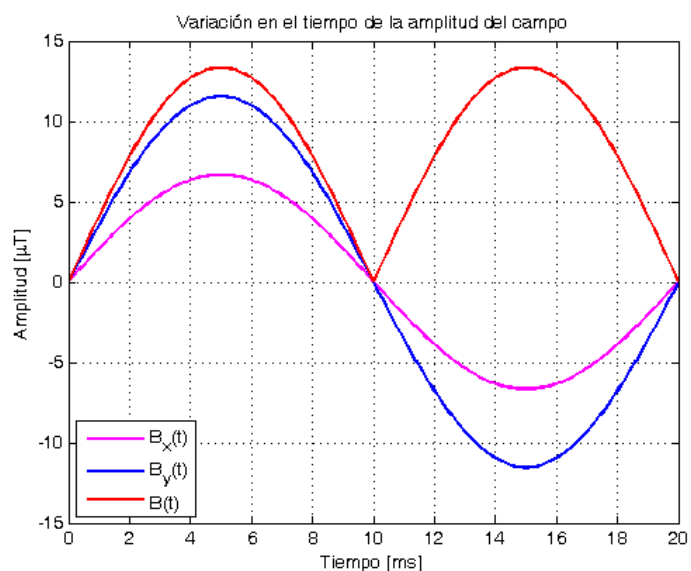

a)Variación temporal

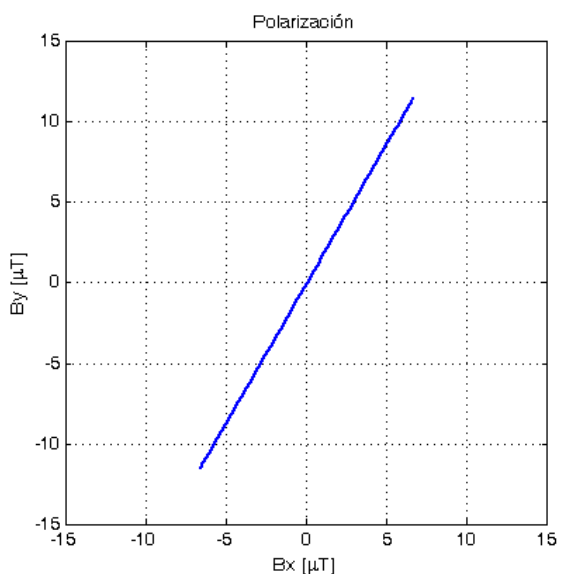

a)Variación espacial

Fig. AIII 6 - Ejemplo de polarización lineal, $\mathrm{B}_{\mathrm{xmàx}} \neq \mathrm{B}_{\mathrm{xmàx}} \mathrm{y} \varphi_{\mathrm{xy}}=0$ 


\section{AIII-2. Ejemplos de polarización del campo magnético}

En este inciso se presentan algunas instalaciones típicas, para las que se calculan los perfiles de campo magnético y la polarización en el centro de la línea $(0 \mathrm{~m})$, a 5, 10, 20 y 40m desde el centro de la línea. Todos los cálculos se realizaron a $1 \mathrm{~m}$ sobre el nivel del suelo.

En la Fig. AIII 7 se presentan los resultados para una LAAT de 500 kV, la estructura utilizada corresponde a la "V Arriostrada", en a) se muestra la torre con la disposición de conductores y en b) el perfil de campo con la polarización para los diferentes puntos.

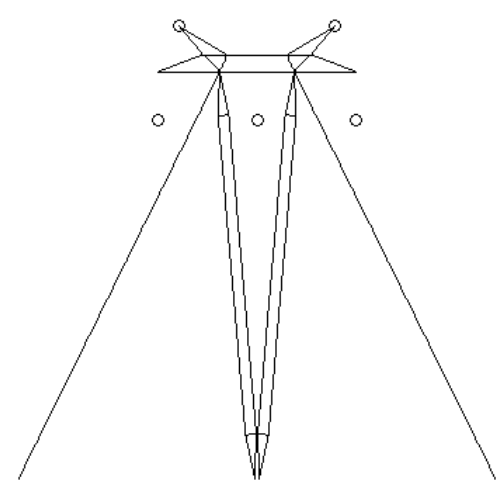

a)Torre V Arriostrada

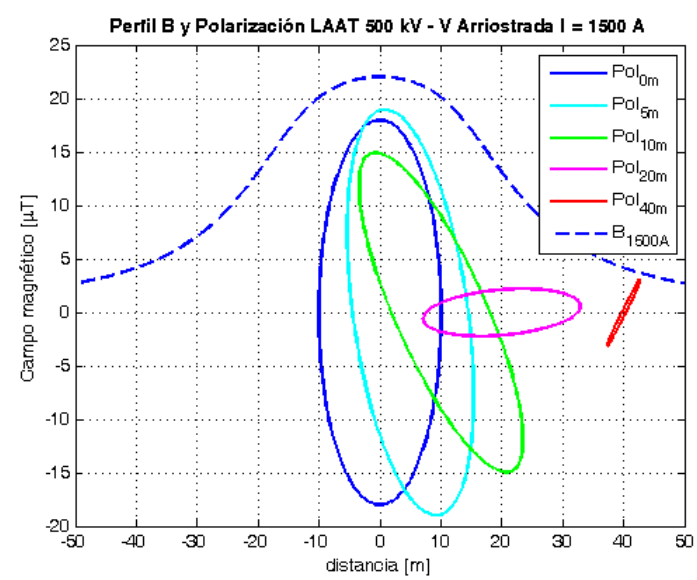

a)B y Polarización

Fig. AIII 7 - Ejemplo de polarización LAAT 500 kV “V Arriostrada”.

En la Fig. AIII 8 se presentan los resultados para una LAAT de 500 kV, la estructura utilizada corresponde a la "Cross-Rope", en a) se muestra la torre con la disposición de conductores y en b) el perfil de campo con la polarización para los diferentes puntos.

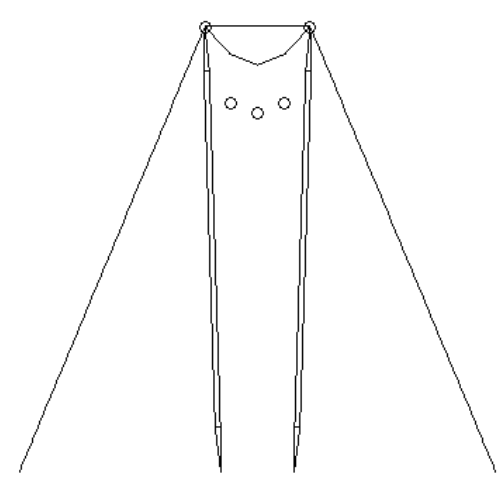

a)Torre Cross-Rope

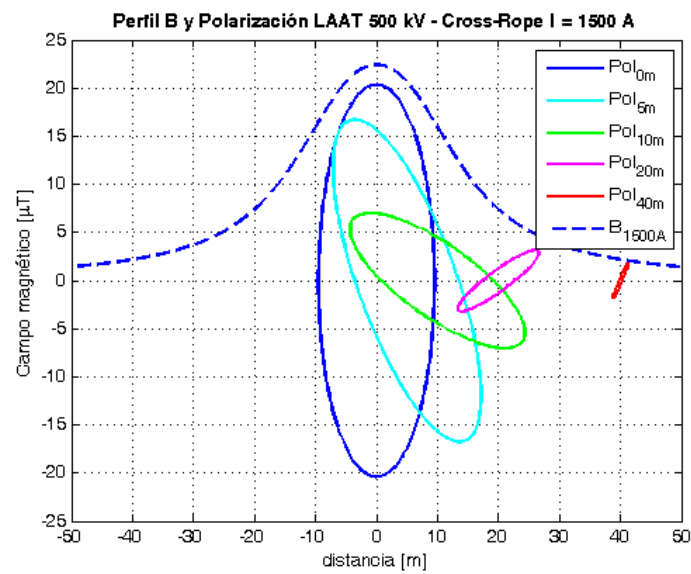

a)B y Polarización

Fig. AIII 8 - Ejemplo de polarización LAAT 500 kV V “Cross-Rope”.

En la Fig. AIII 9 se presentan los resultados para un CSAT de $220 \mathrm{kV}$, la configuración utilizada es trébol y corresponde a la zona de ductos, en a) se muestra la disposición de conductores y en b) el perfil de campo con la polarización para los diferentes puntos. 


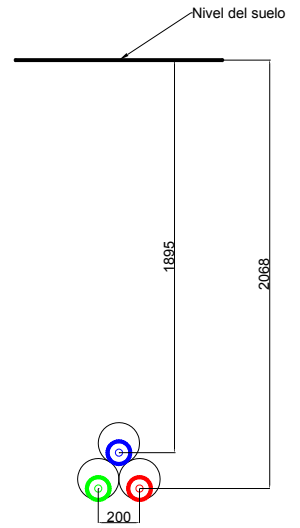

a)Zona de ductos

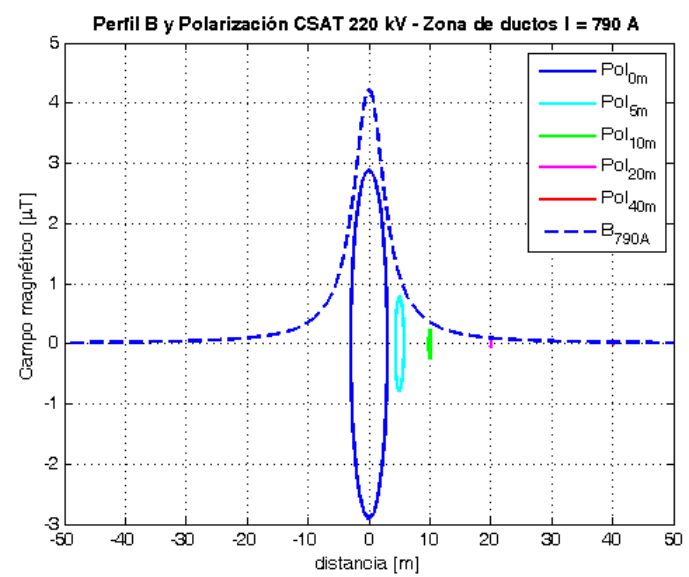

a)B y Polarización

Fig. AIII 9 - Ejemplo de polarización CSAT 220 kV, zona de ductos

En la Fig. AIII 10 se presentan los resultados para un CSAT de $220 \mathrm{kV}$, la configuración utilizada es coplanar horizontal y corresponde a la zona de empalmes, en a) se muestra la disposición de conductores y en b) el perfil de campo con la polarización para los diferentes puntos.

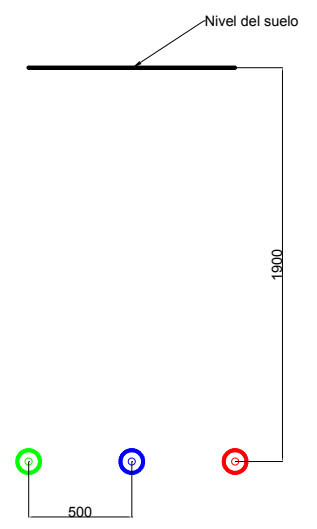

a)Zona de empalmes

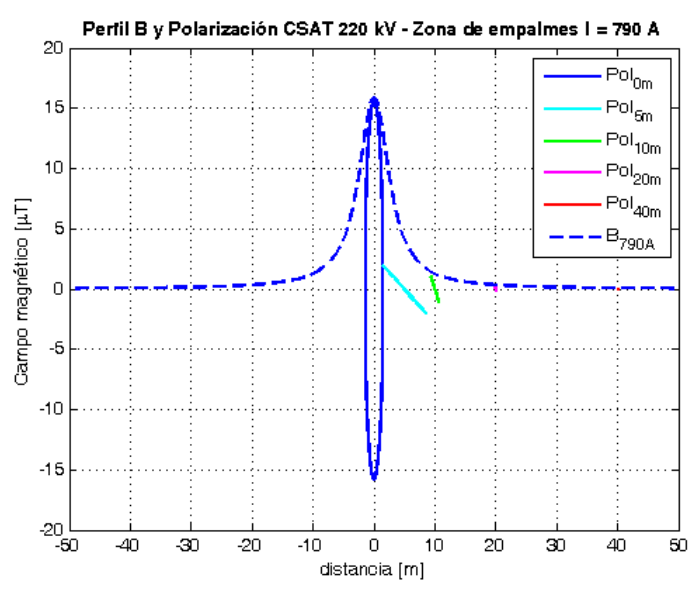

a)B y Polarización

Fig. AIII 10 - Ejemplo de polarización CSAT 220 kV, zona de empalmes 
En la Fig. AIII 11 se presentan los resultados para un LAAT de 2 x 220 kV, la configuración utilizada es coplanar vertical, en a) se muestra la disposición de conductores, en la misma se aprecia que no existe trasposición de fases y en b) el perfil de campo con la polarización para los diferentes puntos.

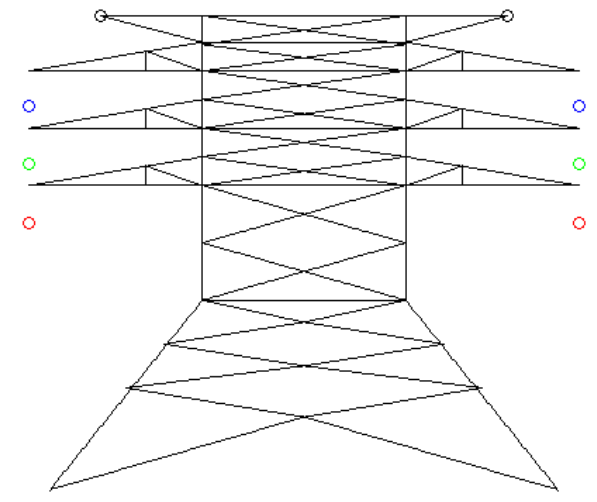

a)Estructura y trasposición de conductores

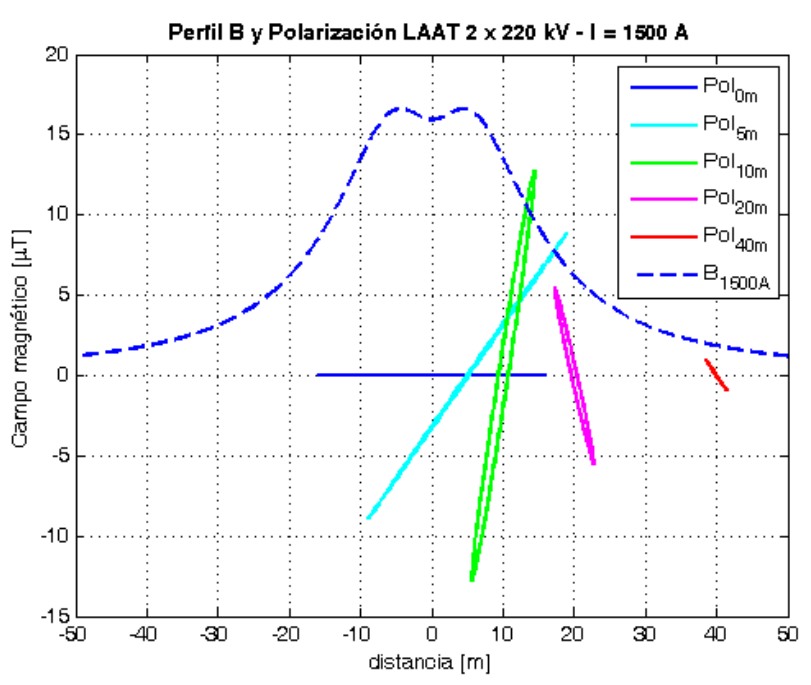

b)B y Polarización

Fig. AIII 11 - Ejemplo de polarización LAAT 2 x 220 kV, sin trasposición de fases.

En la Fig. AIII 12 se presentan los resultados para un LAAT de 2 x 220 kV, la configuración utilizada es coplanar vertical, en a) se muestra la disposición de conductores, en la misma se aprecia que estos se encuentran traspuestos y en b) el perfil de campo con la polarización para los diferentes puntos.

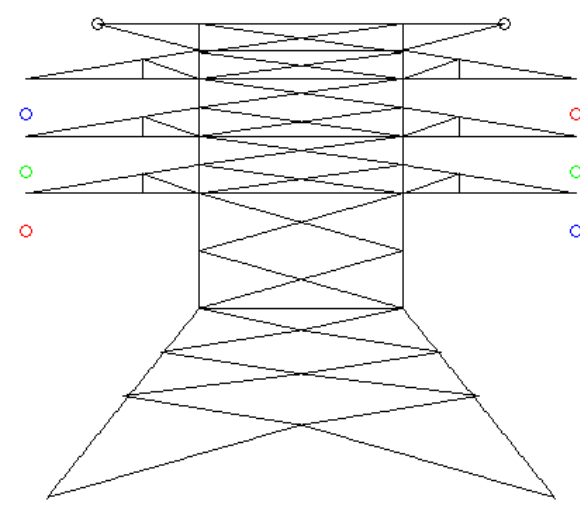

a) Estructura y trasposición de conductores

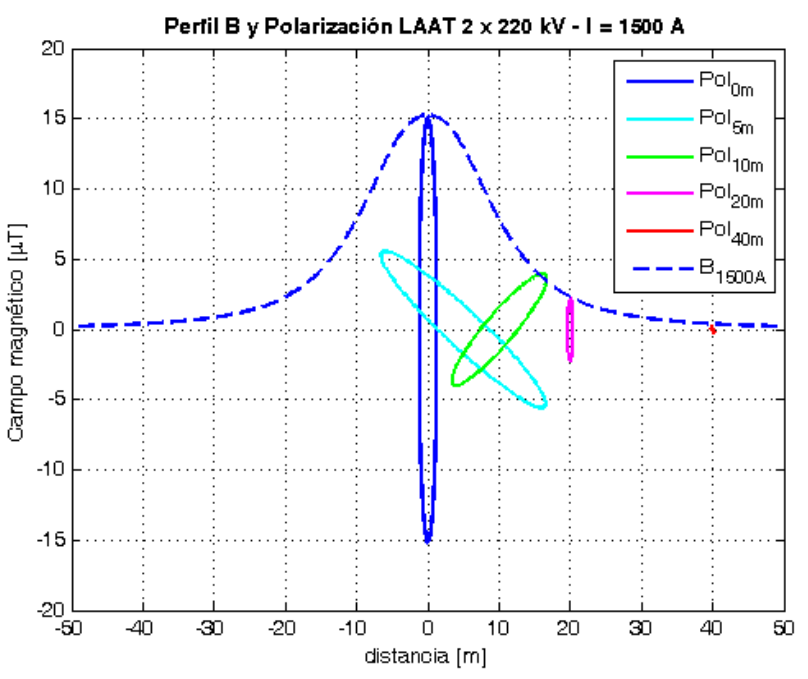

a)B y Polarización

Fig. AIII 12 - Ejemplo de polarización LAAT 2 x 220 kV, con trasposición de fases. 


\section{Anexo IV}

Descripción del software comercial - Opera 3D ELEKTRA SS

Condiciones de borde

Condiciones de borde en caso de simetría 


\section{AIV-1. Descripción del software comercial - Opera 3D ELEKTRA SS}

El software Opera 3D, utilizado para cálculos de campo magnético en tres dimensiones, posee diferentes módulos que permiten resolver diferentes problemas, utilizando el método de los elementos finitos o bien por integración directa.

El módulo que se emplea para el cálculo de campos magnéticos de frecuencias extremadamente bajas es el ELEKTRA SS. Este se puede utilizar para calcular campos electromagnéticos variables en el tiempo en tres dimensiones, incluyendo los efectos de corrientes de Foucault. No modela los efectos de corrientes de desplazamiento. Prácticamente, esto significa que la dimensión más grande del dispositivo que está siendo modelado debe ser inferior a 1/10 de la longitud de onda del campo electromagnético en el espacio libre. El programa incorpora algoritmos para el cálculo de los campos electromagnéticos y procedimientos de análisis numéricos utilizando el método de los elementos finitos.

Los campos electromagnéticos de baja frecuencia se describen las ecuaciones de Maxwell, que excluyen corrientes de desplazamiento.

El campo magnético producido por una distribución conocida de la corriente en el espacio libre (por ejemplo, el campo producido por una bobina realizada con un alambre fino que transporta una corriente especificada) se puede calcular mediante la integración de la ecuación de Biot-Savart.

ELEKTRA SS admite dos tipos de conductores, como fuente de campo magnético:

- Fuente de corriente (Biot-Savart): estos conductores son segmentos de corriente, sus campos se calculan por integración. Son independientes de la malla de elementos finitos.

- Elemento de circuito: estos conductores se incorporan en la malla de elementos finitos, representada por filamentos de bobinados de y están conectados a los circuitos externos. Los circuitos pueden ser fuentes de tensión o corriente y también pueden contener componentes pasivos tales como inductores y capacitores.

Para los casos presentados se utilizaron únicamente segmentos de corriente como fuentes de campo magnético. 


\section{AIV-2. Condiciones de borde}

Las condiciones de contorno se utilizan de dos maneras. En primer lugar pueden proporcionar una forma de reducir el tamaño de la representación de elementos finitos de los problemas simétricos. En segundo lugar se utilizan para aproximar el campo magnético a grandes distancias del problema (campo lejano, límites).

La simetría del problema y la simetría de los campos están implícitas en las potenciales condiciones de contorno aplicadas al modelo de elementos finitos. Los tipos más simples de condiciones de contorno son las que se indican en la Tabla AIV 1.

Tabla AIV 1 - Condiciones de borde ELEKTRA SS - Opera 3D

\begin{tabular}{|l|l|l|}
\hline \multicolumn{3}{|c|}{ Condiciones de borde } \\
\hline Condición & Simetría del campo & Vector Potencial \\
\hline $\begin{array}{l}\text { Tangencial Magnético } \\
0\end{array}$ & $H \sqsubset n=0$ & $A \times n=0$ \\
Normal Eléctrico & $E \times n=0$ & $V=0$ \\
\hline $\begin{array}{l}\text { Normal Magnético } \\
\text { O } \\
\text { Tangencial Eléctrico }\end{array}$ & $H \times n=0$ & $(\nabla \times A) \times n=0$ \\
\hline
\end{tabular}

donde " $n$ " es el vector unitario normal a la superficie. Un valor distinto de cero para el potencial eléctrico escalar $\mathrm{V}$ en una superficie externa se puede utilizar para conducir corriente en un problema.

Las condiciones de contorno que se muestran en la Tabla AIV 1 anterior sólo se aplican a las superficies exteriores al modelo de elementos finitos.

\section{AIV-3. Condiciones de borde en caso de simetría}

En algunos casos es posible aprovechar la simetría que presenta el problema y reducir el tamaño de la malla de elementos finitos. A continuación se presenta una descripción de del procedimiento que se utiliza para imponer condiciones de borde en estos casos.

En la Fig. AIV 1 se presenta el caso en donde se elige un plano de simetría (Superficie con condición de borde), se tienen dos conductores equidistantes al plano de simetría, las corrientes por los conductores tienen la misma magnitud (Ia=Ib) y están en fase. En esta condición se analizan las componentes del campo "B", producido por ambos conductores, sobre la superficie, en donde se pretende aplicar la condición de borde. "Ba" es el campo producido por la corriente "Ia", se representa en color rojo. Las componentes en el eje x e y son "Bax" y "Bay" se representan en el mismo color y en línea de trazos. "Bb" es el campo producido por la corriente " $\mathrm{Ib}$ ", se representa en color azul. Las componentes en el eje x e y son "Bbx" y "Bby" se representan en el mismo color y en línea de trazos.

En esta condición se observa que las componentes en el eje " $\mathrm{x}$ " (Bax y Bbx) se anulan, ya que poseen la misma magnitud pero sentidos opuestos. El campo resultante es normal a la superficie de simetría. Por lo que es posible imponen sobre esta superficie la condición de que el campo B sea normal. 
Fig. AIV 1 - Aplicación de condición de borde normal magnético en ELEKTRA SS - Opera 3D.

En la Fig. AIV 2 se presenta el caso en donde se elige un plano de simetría (Superficie con condición de borde), se tienen dos conductores equidistantes al plano de simetría, las corrientes por los conductores tienen la misma magnitud ( $\mathrm{Ia}=\mathrm{Ib})$ y están desfasadas $180^{\circ}$. En esta condición se analizan las componentes del campo "B", producido por ambos conductores, sobre la superficie, en donde se pretende aplicar la condición de borde. "Ba" es el campo producido por la corriente "Ia", se representa en color rojo. Las componentes en el eje x e y son "Bax" y "Bay" se representan en el mismo color y en línea de trazos. "Bb" es el campo producido por la corriente "Ib", se representa en color azul. Las componentes en el eje $\mathrm{x}$ e y son "Bbx" y "Bby" se representan en el mismo color y en línea de trazos.

En esta condición se observa que las componentes en el eje "y" (Bay y Bby) se anulan, ya que poseen la misma magnitud pero sentidos opuestos. El campo resultante es tangencial a la superficie de simetría. Por lo que es posible imponen sobre esta superficie la condición de que el campo B sea tangencial.

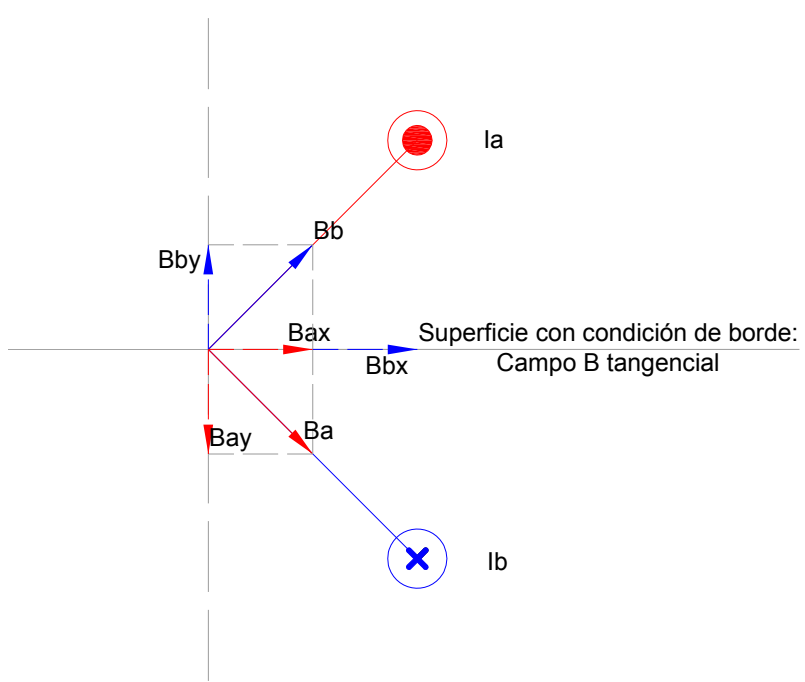

Fig. AIV 2 - Aplicación de condición de borde tangencial magnético en ELEKTRA SS - Opera 3D. 
Datos geométricos de LAAT y CSAT 


\section{AV-1. Datos geométricos de LAAT y CSAT}

En este inciso se presentan los datos geométricos de las configuraciones utilizada para LAAT y CSAT. En el caso de LAAT la altura de de los conductores sobre el terreno se indica en cada punto en particular, ya que en algunos casos se considero la altura de los conductores en el momento de las mediciones y en otros casos se consideraron otras alturas, como por ejemplo la altura mínima para cada tipo de línea adoptada por la reglamentación vigente.

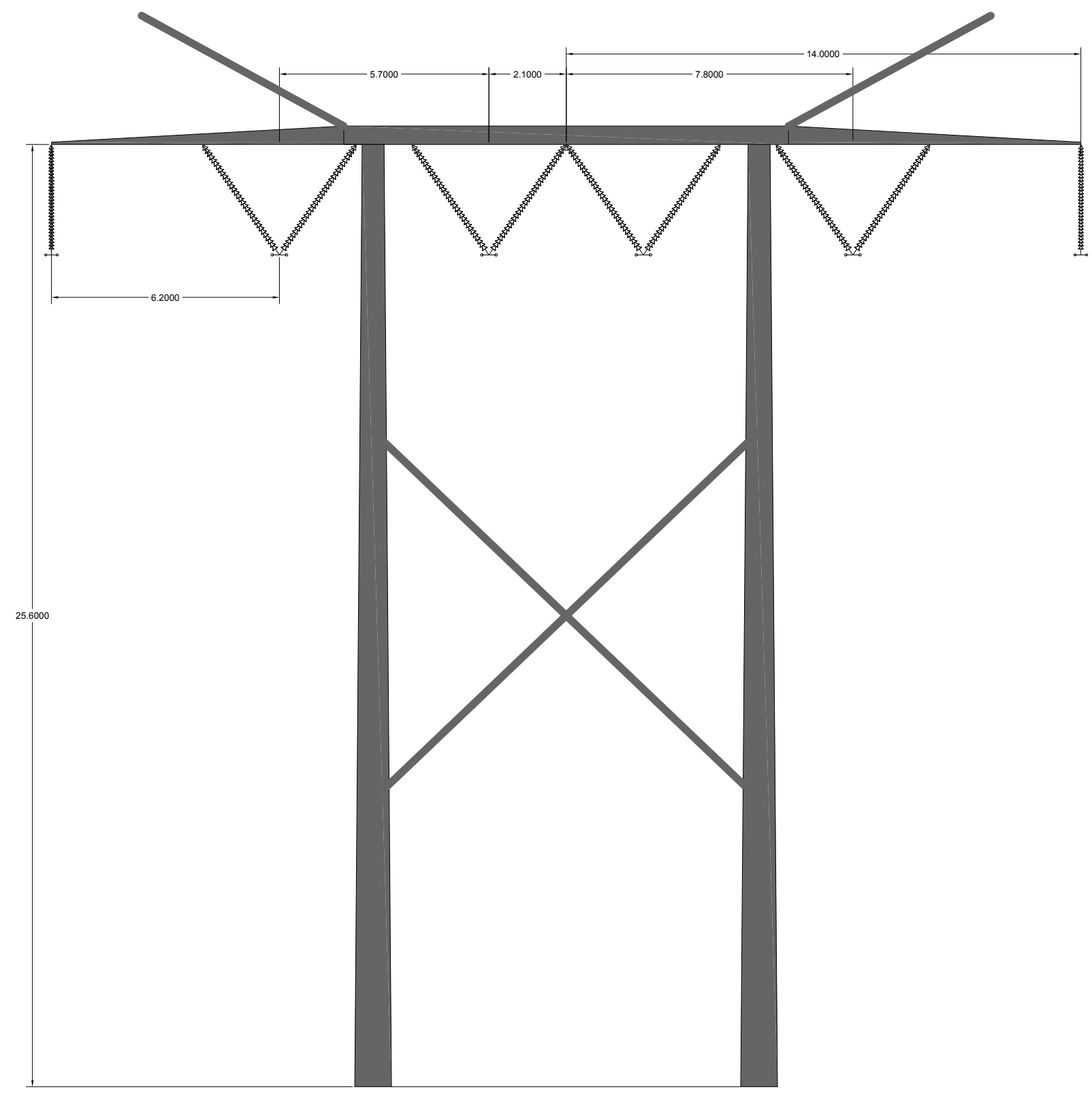

Fig. AV 1 -LAAT 2 x 220 kV, coplanar horizontal con aisladores de suspensión (CH-AS). 


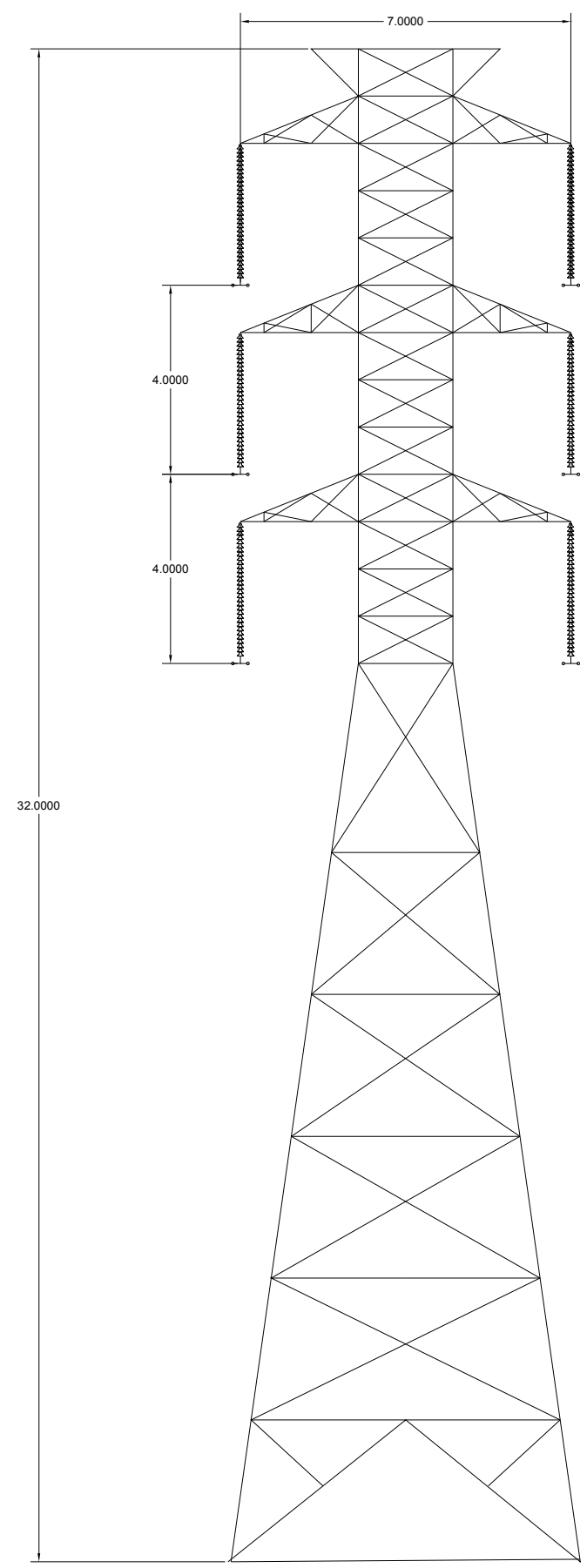

Fig. AV 2 -LAAT 2 x 220 kV, coplanar vertical con aisladores de suspensión (CV-AS). 


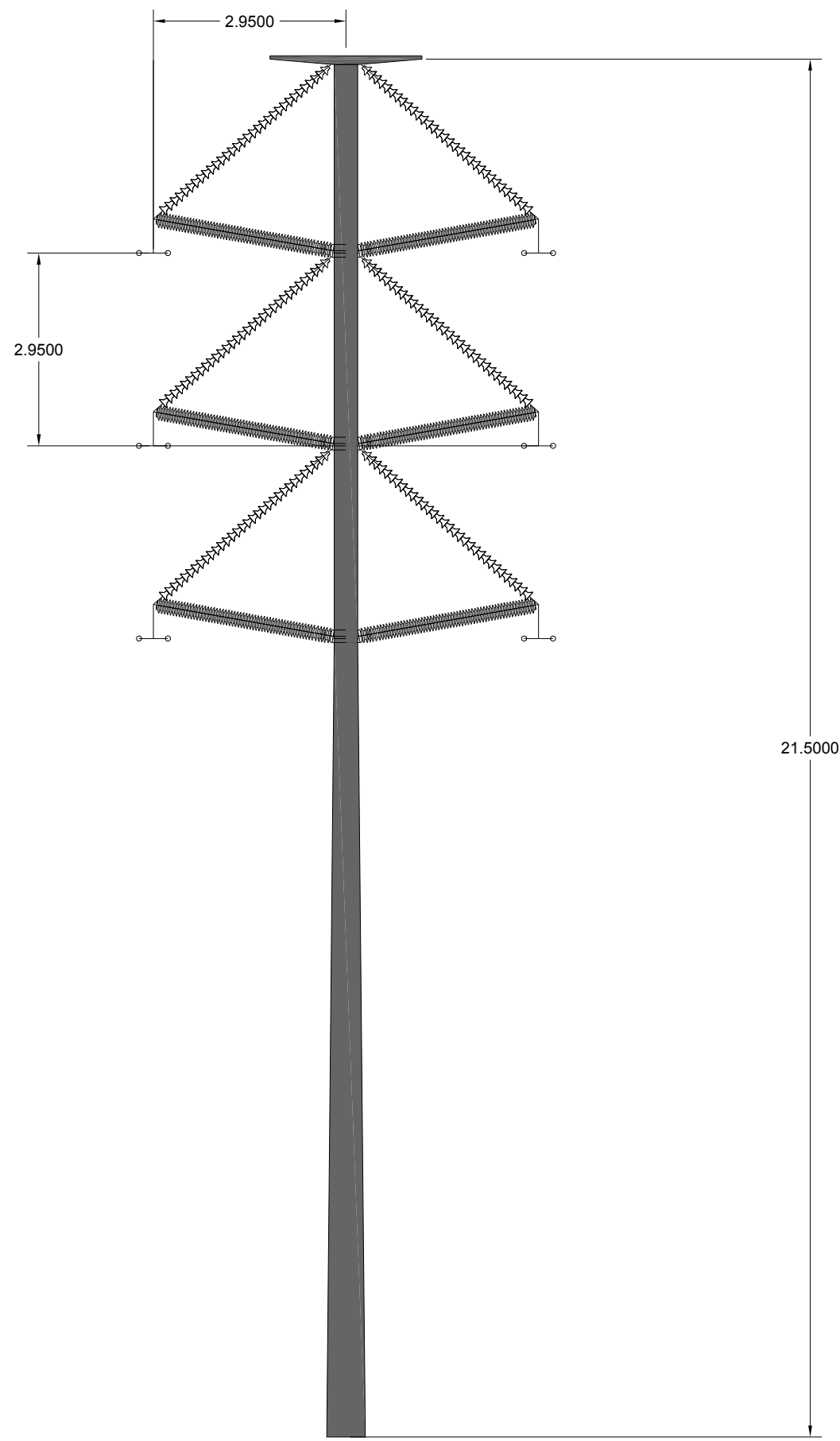

Fig. AV 3 -LAAT 2 x 220 kV, coplanar vertical con aisladores Line Post (CV-LP). 


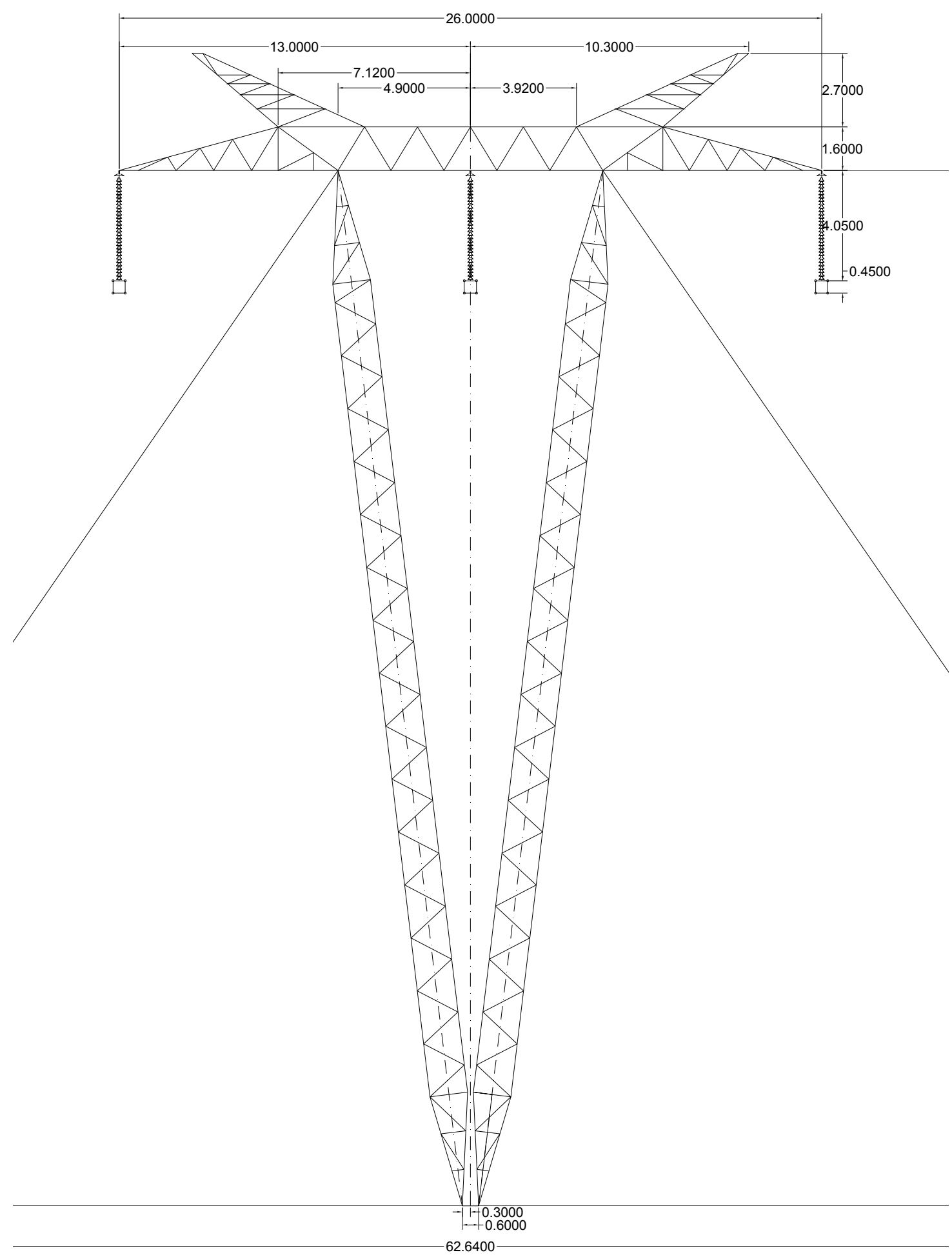

Fig. AV 4 -LAAT 500 kV, coplanar horizontal con aisladores de suspensión, Torre VArriostrada. 


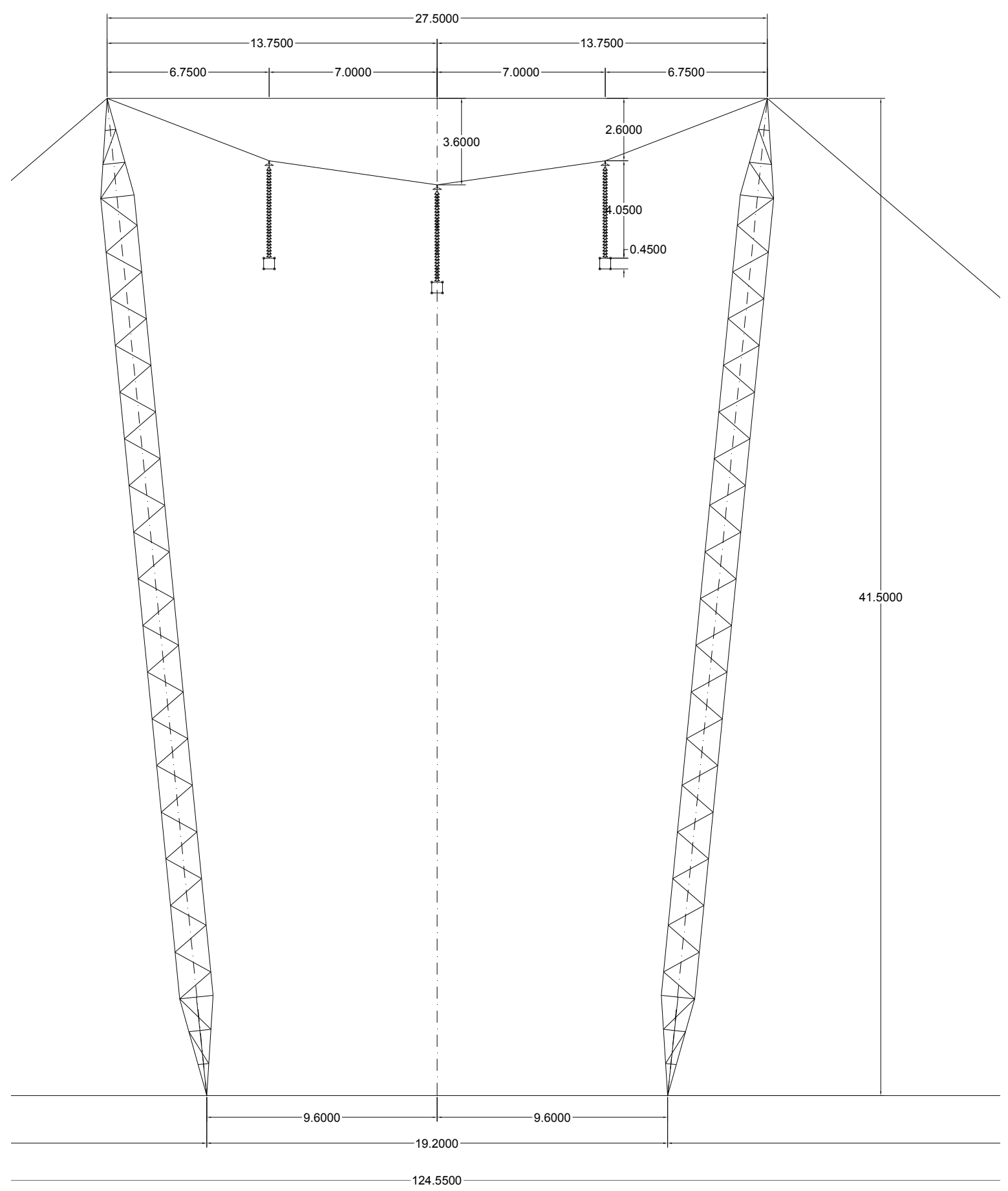

Fig. AV 5 -LAAT 500 kV, coplanar horizontal con aisladores de suspensión, Torre Cross Rope. 

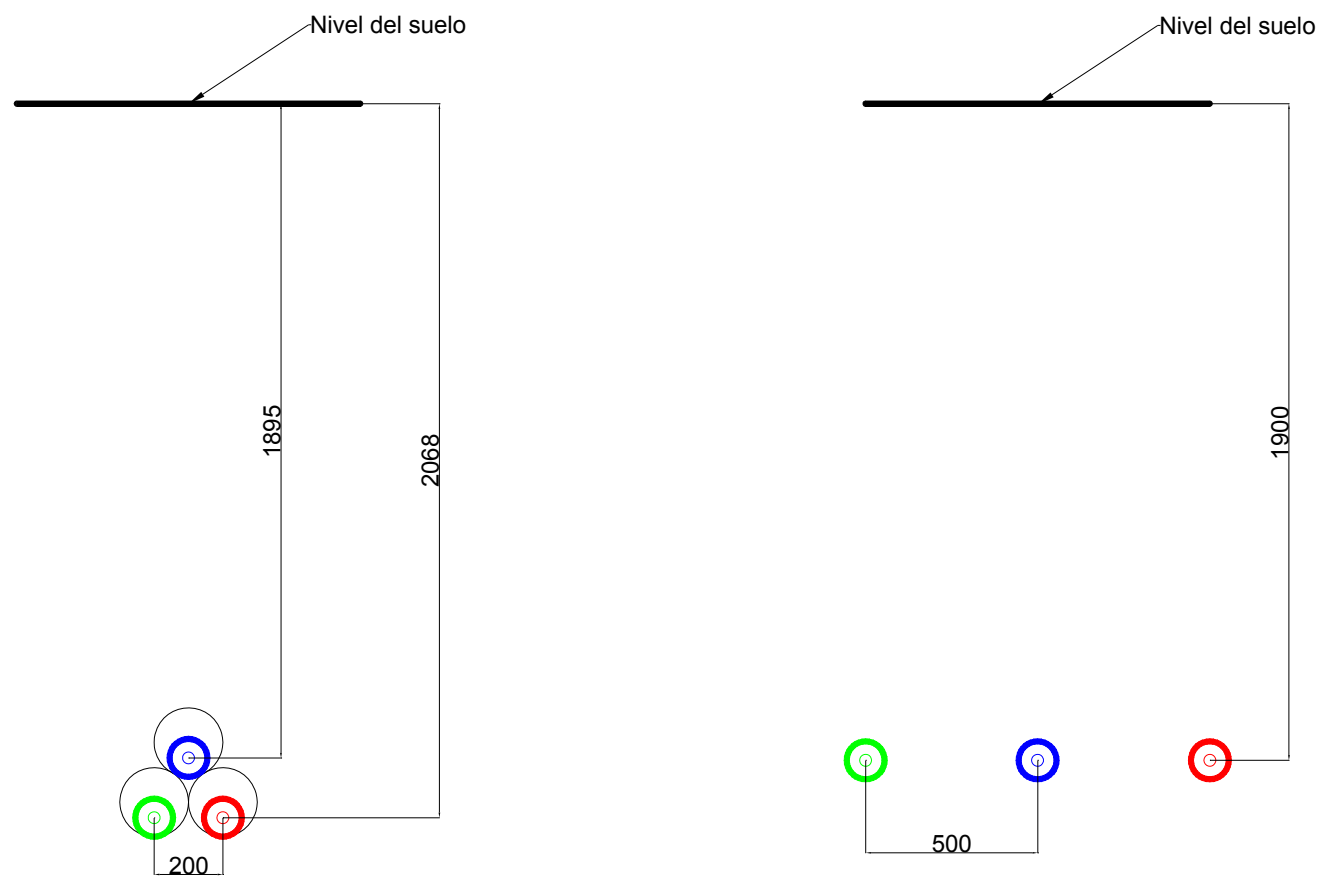

a)Zona de ductos

b)Fosa de empalmes

Fig. AV 6 -CSAT $220 \mathrm{kV}$ zona de ductos y fosa de empalmes

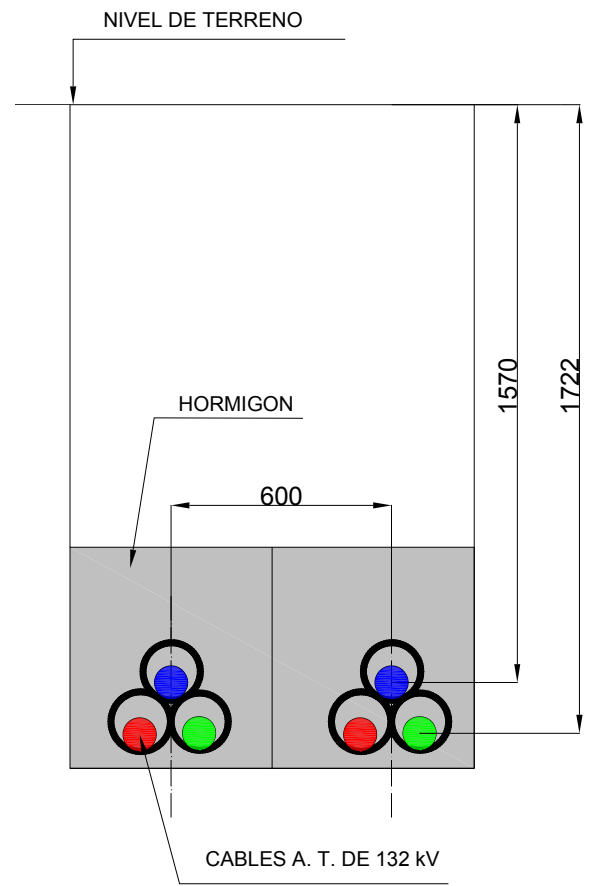

a)Zona de ductos

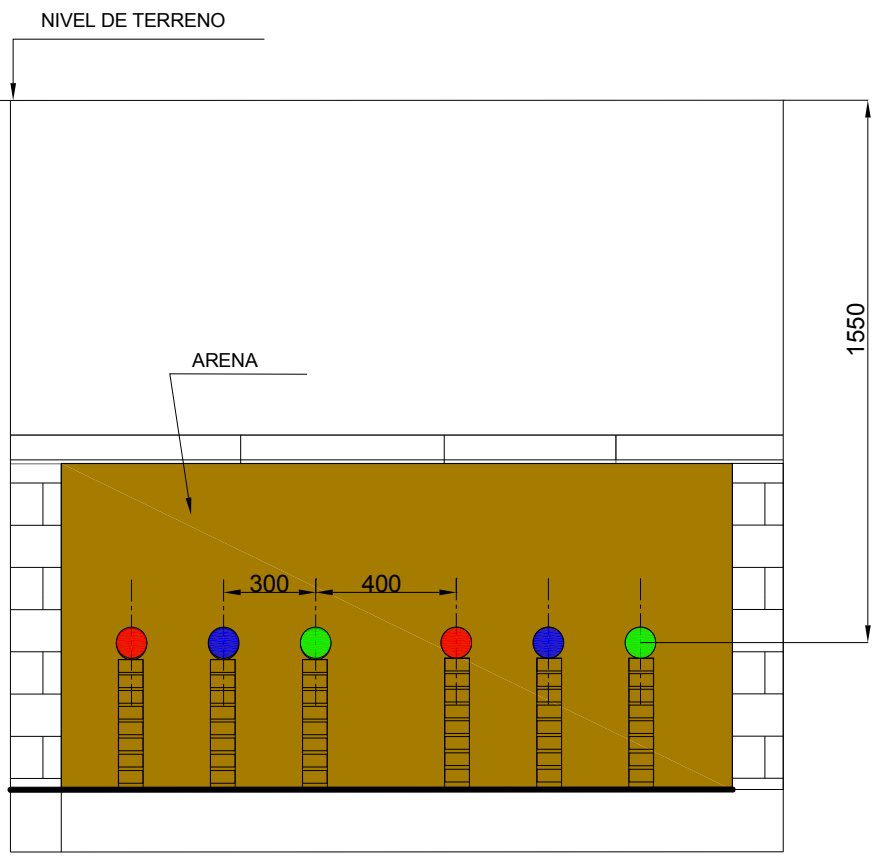

b)Fosa de empalmes

Fig. AV 7 -CSAT 2 x $132 \mathrm{kV}$ zona de ductos y fosa de empalmes 


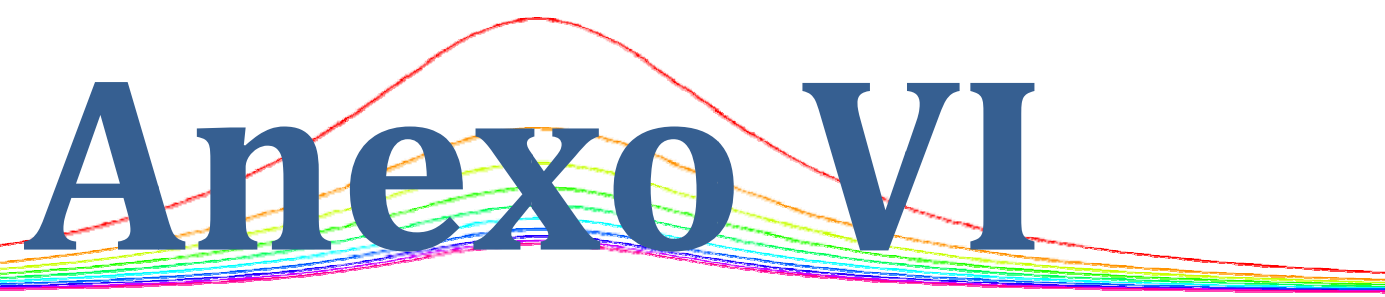

Medición de campo magnético

Calibración de medidores de campo magnético 


\section{AVI-1. Medición de campo magnético}

En la República Argentina para reglamentar los procedimientos de medición de campo magnético, se promulga la Resolución ENRE 1724/98 [ 45 ]. Esta indica que se deben seguir las recomendaciones que da la norma IEEE 644-1994 [ 10 ]. Otra referencia en términos de medición de campo magnético es la norma IEC 61786:1998 [ 28 ]. Estas normas sugieren dos formas distintas de cuantificar los campos, una para los medidores de un solo eje (con una sola bobina) y otra para los medidores de tres ejes, con tres bobinas ortogonales entre sí, en ambos casos se supone que la variación de las componentes del campo es del tipo senoidal.

Según las normas, la indicación de un medidor de tres ejes, llamada campo resultante $\left(B_{R}\right)$, queda definida según la Ec. AVI 1.

$$
B_{R}=\sqrt{B_{X}^{2}+B_{Y}{ }^{2}+B_{Z}^{2}}
$$

En donde se deben obtener las tres componentes espaciales que definen al campo: $\mathrm{B}_{\mathrm{X}}, \mathrm{B}_{\mathrm{Y}} \mathrm{y}$ $\mathrm{B}$.

En la Fig. AVI 1 se presenta el corte de una zonda de $100 \mathrm{~cm}^{2}$, de un medidor de campo de tres ejes, marca Narda, modelo EFA 300. En esta se aprecian las tres bobinas utilizadas para la medición de campo en cada uno de los ejes.

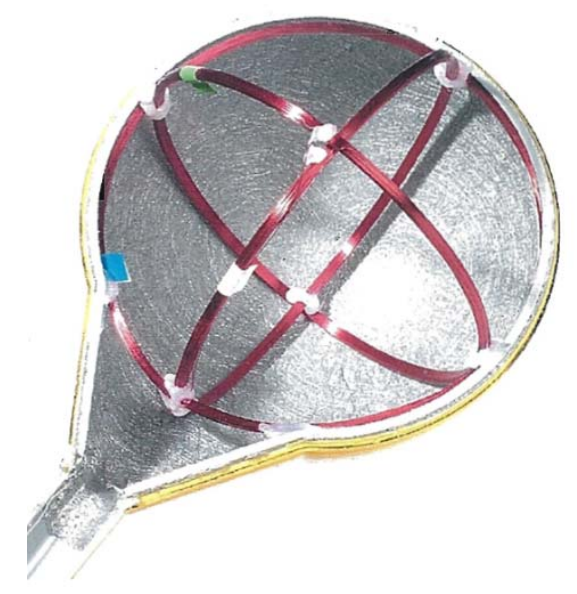

Fig. AVI 1 - Corte de zonda de medición de campo magnético, utilizada en medidores tridimensionales, Narda EFA 300.

En algunas instalaciones como ser Línea Aéreas o cables subterráneos, si se considera que estos están tendidos en la dirección de uno de los ejes (X, Y o Z), se puede decir que no existe componente de campo en la dirección de la línea, por lo que la Ec. AVI 1 puede simplificarse eliminando una de las componentes, como se presenta en la Ec. AVI 2, donde se considera a la línea tendida a lo largo del "eje Z".

$$
B_{R}=\sqrt{B_{X}{ }^{2}+B_{Y}{ }^{2}}
$$

Los medidores de un eje pueden ser usados para obtener el máximo valor del campo magnético, orientando la bobina hasta obtener la lectura del mayor valor de campo.

Es posible calcular el campo magnético $B_{R}$ a partir de tres mediciones utilizando un medidor de un eje. Con mediciones secuenciales de las tres componentes, orientando el sensor en tres 
direcciones ortogonales, tomando lecturas en cada una de ellas y luego realizando la composición de acuerdo con la Ec. AVI 1 se obtiene el campo resultante. Para realizar esta operación debe garantizarse que el campo no esté variando temporalmente.

Si se miden campos elíptica o circularmente polarizados, el campo resultante $\mathrm{B}_{\mathrm{R}}$ puede ser mayor que el campo máximo. La mayor diferencia se encuentra en el caso de polarización circular, en cuyo caso $B_{R}$ excede en un 41\% al campo máximo [ 10 ].

En la Fig. AVI 2 y Fig. AVI 3 se presentan imágenes de instrumentos de medición de campo magnético de tres ejes. El primero marca HIOKI, modelo FT3470-52 y en segundo lugar uno marca NARDA modelo EFA 300. En ambas figuras se aprecian los instrumentos con la zonda e interfaz con el usuario.

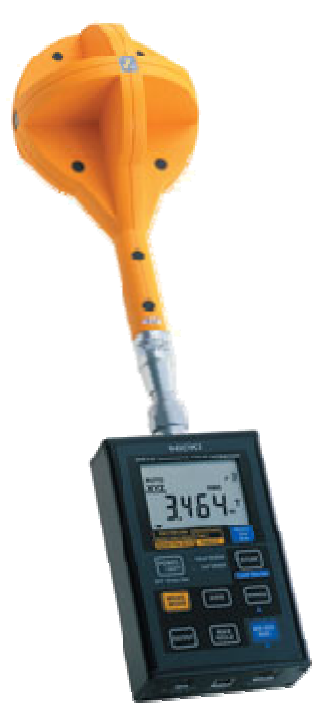

Fig. AVI 2- Instrumento de medición de campo magnético HIOKI.

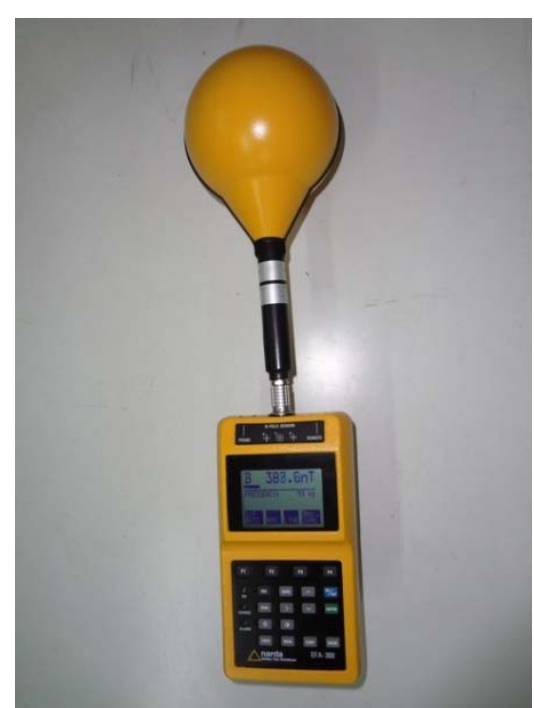

Fig. AVI 3 - Instrumento de medición de campo magnético NARDA EFA 300.

\section{AVI-2. Calibración de medidores de campo magnético}

En la Fig. AVI 4 se presenta el medidor de campo magnético, introducido en una bobina de Helmoltz, esta bobina posee dos espiras cuadradas con 1,26 m de lado y una separación entre ambas de 0,76 m. Esta bobina se utiliza como elemento de calibración de campo magnético, ya que en centro de la misma es posible generar un campo magnético constante, cuyo valor es proporcional a la corriente por las espiras. La utilización de este tipo de elementos, con propósito de calibración, está contemplado en las normas IEEE 644-1994 [ 10 ] e IEC 61786:1998 [ 28 ]. 


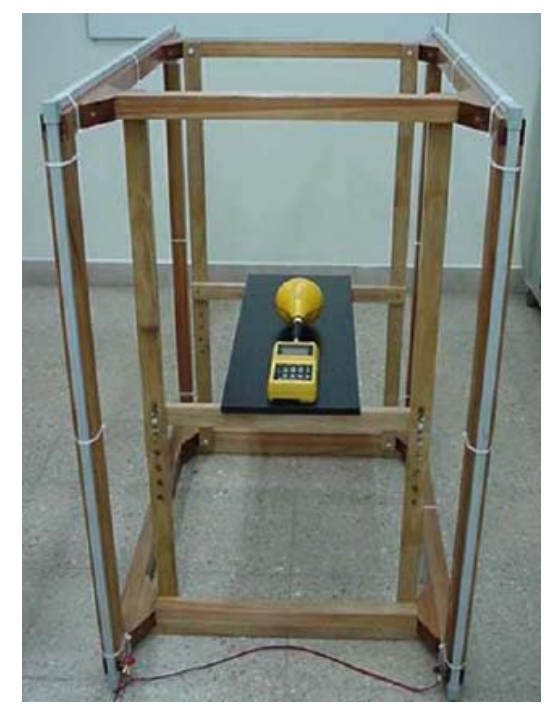

Fig. AVI 4 - Instrumento de medición de campo magnético y bobina de Helmoltz.

En la Fig. AVI 5 se presentan los resultados obtenidos, por medio de una simulación realizada con un modelo tridimensional, en el que se aprecia que en la zona central, a una altura de $0,63 \mathrm{~m}$, el campo magnético es constante.

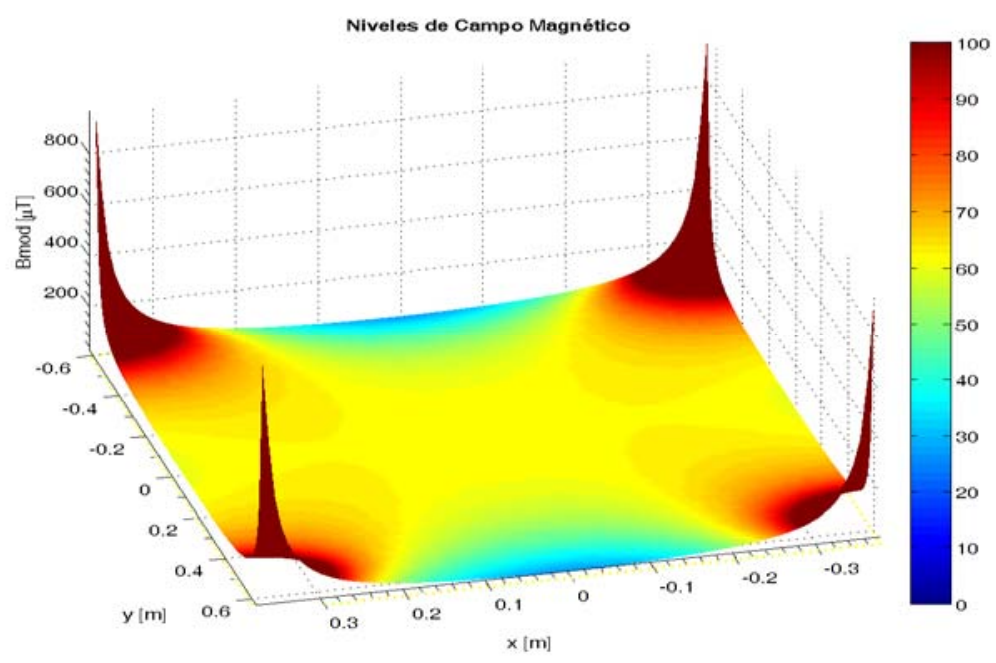

Fig. AVI 5 - Campo magnético en el interior de la bobina de Helmoltz, con una corriente de $51 \mathrm{~A}$ 


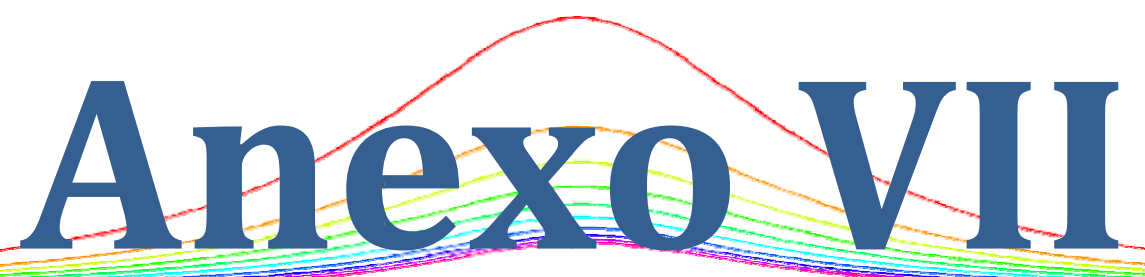

Análisis de sensibilidad a la disposición de conductores - Simple Terna

Análisis de sensibilidad a diferentes secuencias de energización - Doble Terna 


\section{AVII-1. Análisis de sensibilidad a la disposición de conductores - Simple Terna}

Existen diferentes configuraciones para las líneas de energía eléctrica, dependiendo de la distancia entre las fases y la posición relativa, en función estas se obtienen diferentes valores para el campo magnético.

Como ejemplo de esto se puede mencionar disposiciones triangulares o delta, coplanar vertical u horizontal.

A continuación se presentan algunos ejemplos que permiten entender esta situación, se platean en primer lugar los casos sistemas trifásicos balanceados y luego se introducen desbalances con el objeto de evaluar la influencia de estos.

Se consideran por un lado conductores ubicados en un mismo plano con diferentes distancias entre los mismos, 5 y $10 \mathrm{~m}$, por otro lado para las mismas distancias se consideran los conductores dispuestos en delta. Para el caso de la configuración delta la distancia entre los tres conductores es la misma, pero en el caso de los conductores ubicados en un mismo plano la distancia entre los conductores externos es el doble que la de los conductores más próximos.

En la Fig. AVII 1 se presentan curvas de nivel obtenidas cálculos de campo magnético realizados para los casos anteriormente mencionados, considerando una corriente de $2000 \mathrm{~A}$ balanceada. En dicha figura es posible visualizar dos situaciones, por un lado se pueden comparar dos configuraciones similares, con distinta distancia de separación entre fases. Se aprecia en a) y c) dos ternas con configuración coplanar horizontal, en a) la separación entre fases contiguas es de $5 \mathrm{~m}$ y entre las fases externas $10 \mathrm{~m}$. en c) se aprecian la misma situación pero las distancias son $10 \mathrm{~m}$ y $20 \mathrm{~m}$ respectivamente.

Se destaca que la configuración cuya distancia entre fases es menor arroja menores valores de campo magnético.

Lo mismo ocurre si se comparan b) y d), en este caso la configuración es triangular, en b) las distancias entre las fase son $5 \mathrm{~m}$ y en d) las distancias son $10 \mathrm{~m}$.

Por lo tanto para configuraciones similares, en donde lo único que se modifica es la distancia entre fases, se puede concluir que las configuraciones más compactas son las que arrojan mejores resultados. 


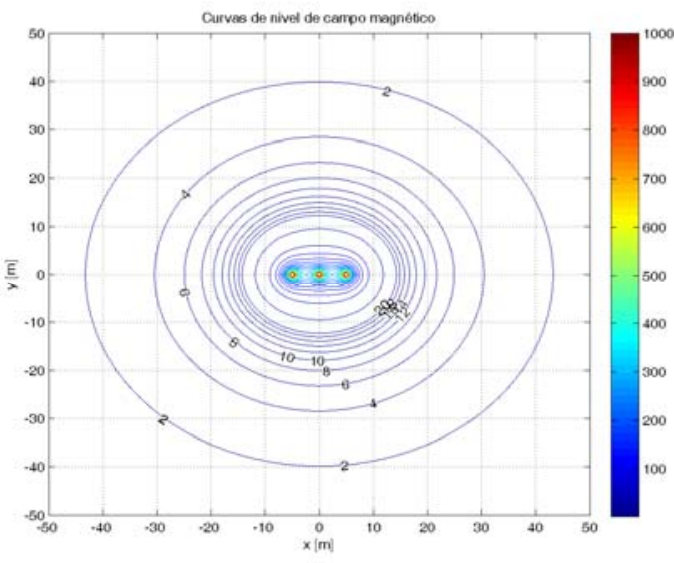

a) Coplanar: $\mathrm{s}=5 \mathrm{~m}$ y $10 \mathrm{~m}$

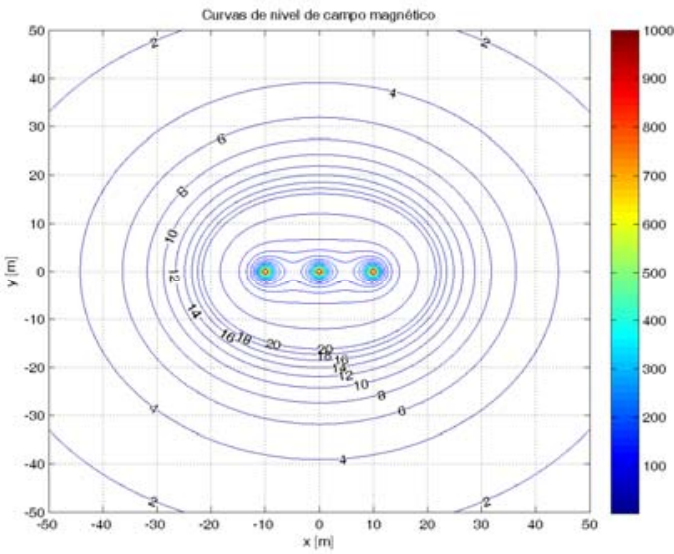

c) Coplanar: $\mathrm{s}=10 \mathrm{~m}$ y $20 \mathrm{~m}$

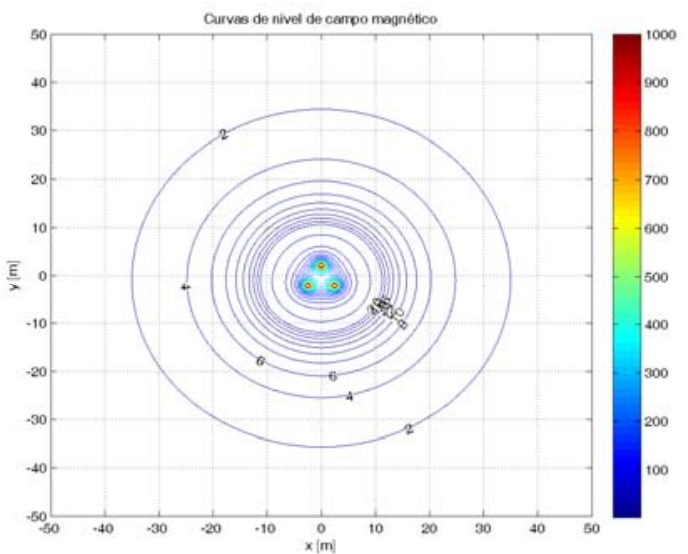

b) Delta: $\mathrm{s}=5 \mathrm{~m}$

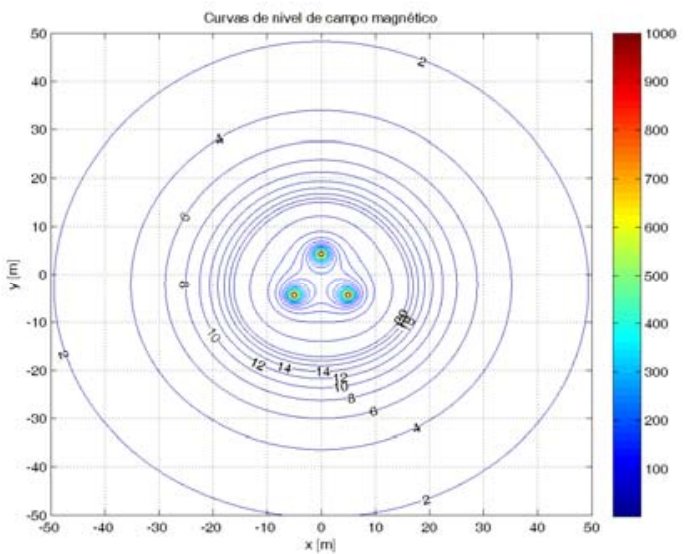

d) Delta: $\mathrm{s}=10 \mathrm{~m}$

Fig. AVII 1 - Curvas de nivel para campo B [ $\mu \mathrm{T}]$, Disposición Coplanar y Delta, I=2000 A balanceada

Es posible realizar otra comparación, considerando diferentes configuraciones, esto es, si se comparan a) y b) o c) y d) se observa que la configuración triangular arroja una mejor distribución de campo magnético, debido a que existe la misma distancia entre los conductores de fase. Es decir la configuración triangular, al ser más compacta, arroja una menor distribución de campo magnético. 
Si se conservan las mismas condiciones geométricas, pero se considera un desbalance de un $5 \%$ en la corriente en uno de los conductores, se obtienen los resultados de la Fig. AVII 2.

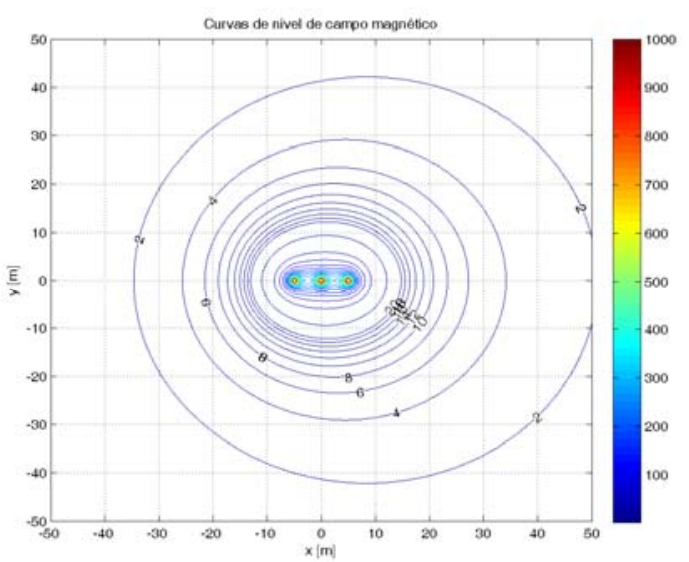

a) Coplanar: $\mathrm{s}=5 \mathrm{~m}$ y $10 \mathrm{~m}$

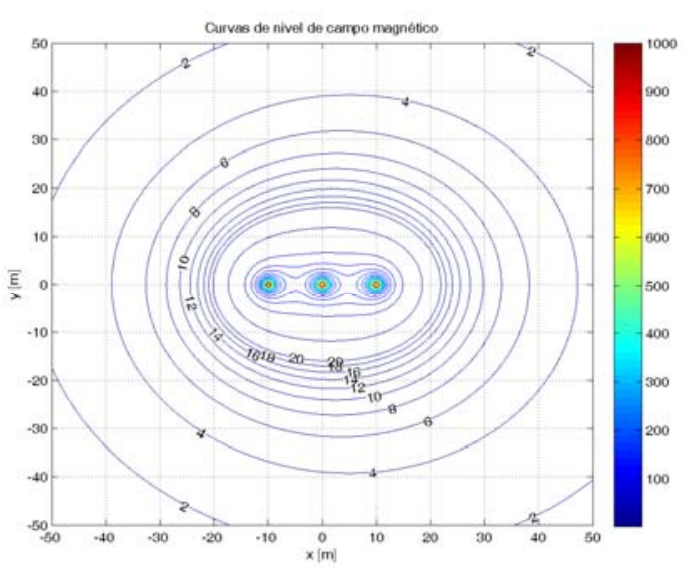

c) Coplanar: $\mathrm{s}=10 \mathrm{~m}$ y $20 \mathrm{~m}$

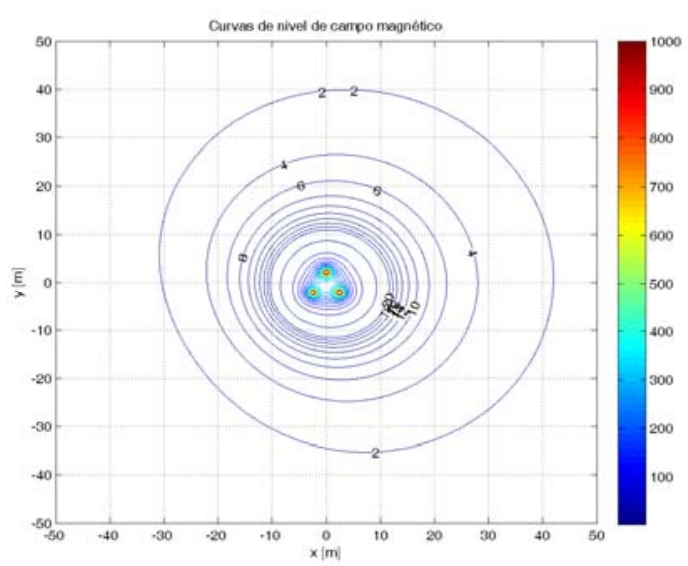

b) Delta: $s=5 \mathrm{~m}$

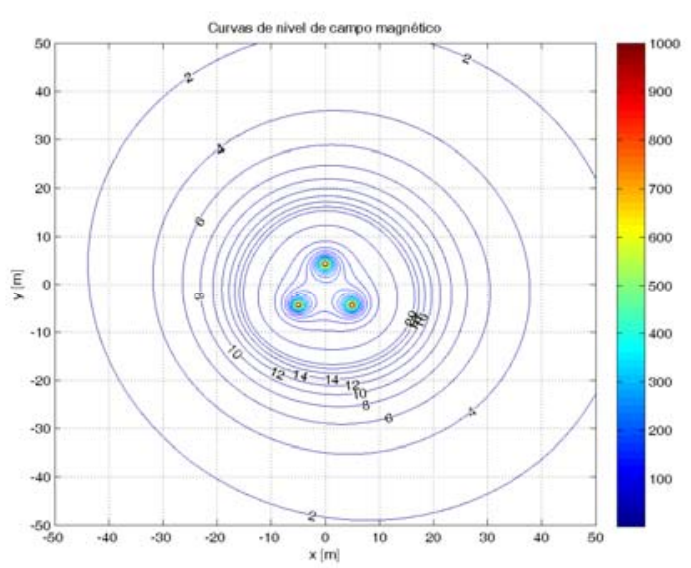

d) Delta: $\mathrm{s}=10 \mathrm{~m}$

Fig. AVII 2 - Curvas de nivel para campo B [ $\mu \mathrm{T}]$, Disposición Coplanar y Delta, I=2000 A desb.: $5 \%$ en un conductor.

Con la introducción de un desbalance se obtienen mayores valores de campo en ciertas áreas a determinadas distancias de los conductores, por ejemplo en " $\mathrm{x}>0$ ", mientras que en otras zonas los valores de campo son inferiores, por ejemplo en " $\mathrm{x}<0$ " para conductores en un mismo plano $\mathrm{y}$ " $\mathrm{x}<0$ e $\mathrm{y}<0$ " para las configuraciones en delta.

Se observa que en presencia de desbalances no es obvia la efectividad de las alternativas de mitigación planteadas, manejo de distancia y configuración de los conductores. Hay que aclarar que la distribución de campo magnético, considerando desbalances guarda relación con la forma en que se produce el desbalance, en este caso se consideró uno de los conductores con una corriente $5 \%$ inferior al de los otros dos, pero si la situación fuera otra debería realizarse el análisis correspondiente.

Para ilustrar esta situación se consideró el desbalance de $5 \%$ en dos conductores, esto es los conductores externos con corrientes $5 \%$ por debajo y por encima respecto del conductor central. Los resultados obtenidos se pueden ver en la Fig. AVII 3. 


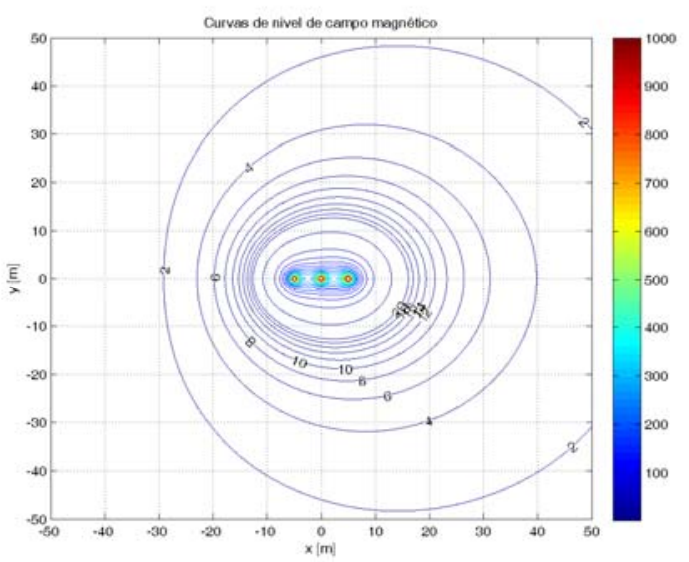

a) Coplanar: $\mathrm{s}=5 \mathrm{~m}$ y $10 \mathrm{~m}$

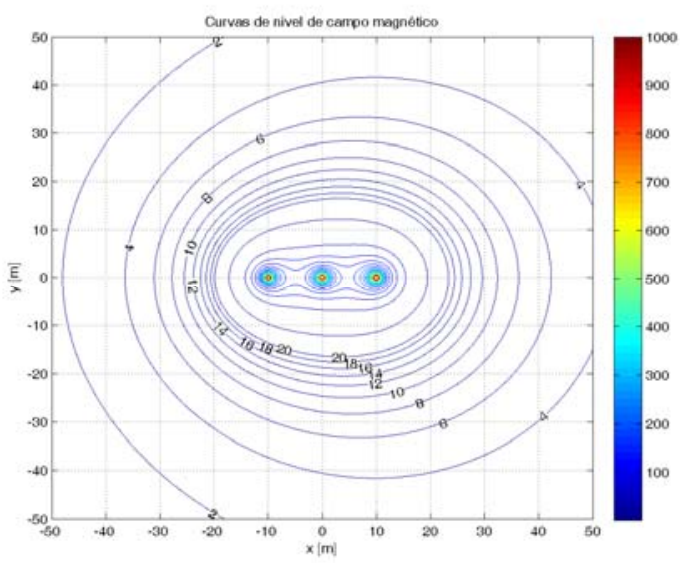

c) Coplanar: $\mathrm{s}=10 \mathrm{~m}$ y $20 \mathrm{~m}$

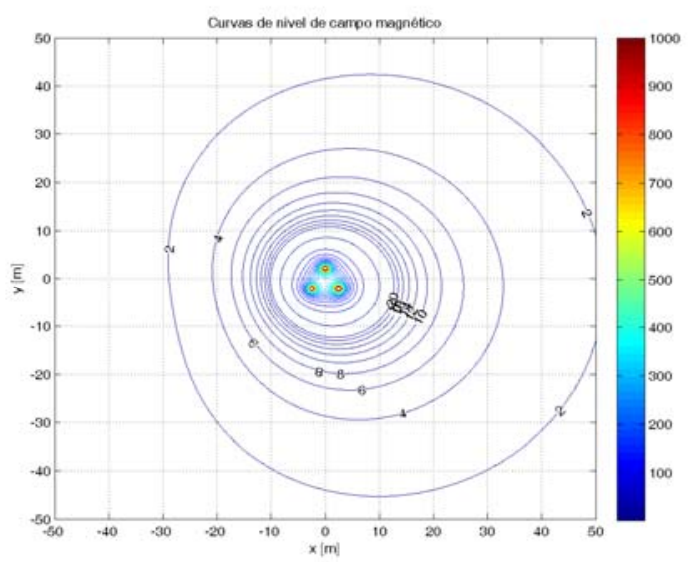

b) Delta: $\mathrm{s}=5 \mathrm{~m}$

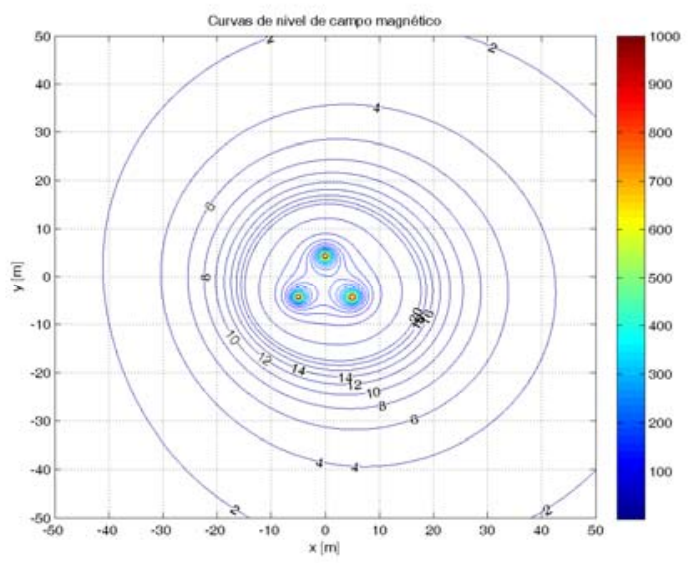

d) Delta: $\mathrm{s}=10 \mathrm{~m}$

Fig. AVII 3 - Curvas de nivel para campo B [ $\mu$ T], Disposición Coplanar y Delta, I=2000 A desb.: $5 \%$ en 2 conductores.

Para estos casos la situación se torna más compleja, ya que las curvas de nivel son más irregulares todavía.

En los casos de los conductores ubicados en un mismo plano se observa que si bien hacia la zona en " $x>0$ " existe un incremento en los valores de campo magnético, en la zona en donde " $\mathrm{x}<0$ " se aprecia una disminución en los valores, respecto a los valores obtenidos para corrientes balanceadas. 


\section{AVII-2. Análisis de sensibilidad a diferentes secuencias de energización - Doble Terna}

Para la aplicación de esta técnica, se consideran dos ternas, cada terna corresponde a circuitos independientes que pueden estar dispuestos sobre una misma estructura. La técnica de mitigación consiste en modificar la posición relativa de las fases. Aquí se considerará a la Terna 1 , constituida por las fases $\mathrm{R}_{1}, \mathrm{~S}_{1}$ y $\mathrm{T}_{1}$ y a la Terna 2 , constituida por las fases $R_{2}, S_{2}$ y $T_{2}$.

En la Fig. AVII 4 se presenta un ejemplo en el que se realiza el cambio en la secuencia de energización de una Terna. En a) se presenta la secuencia de energización convencional. En b) se presenta una alternativa denominada compensada, en este caso se cambian las posiciones de las fases $\mathrm{R}$ y $\mathrm{T}$ de la Terna 2.

\begin{tabular}{|c|c|c|c|c|}
\hline$R_{1} O$ & $\circ R_{2}$ & & $R_{1} O$ & $\circ T_{2}$ \\
\hline$s_{1} \circ$ & $\circ S_{2}$ & $\rightarrow$ & $s_{1} \circ$ & $\circ \mathrm{S}_{2}$ \\
\hline $\mathrm{T}_{1} \circ$ & $\circ T_{2}$ & & $\mathrm{~T}_{1} \mathrm{O}$ & $\circ R_{2}$ \\
\hline
\end{tabular}

Fig. AVII 4 - Ejemplo de cambio en la secuencia de energización de una terna, configuración doble terna.

Los resultados obtenidos para el caso tratado en este punto se presentan en la Fig. AVII 5, en a) se aprecian los valores de campo para el caso de referencia sin desbalance, en b) se muestran los resultados después de realizar el cambio en la secuencia de energización.

Se destaca que en el caso b) las curvas de nivel de campo magnético son mucho más compactas y se encuentran los mayores valores de campo en el entorno de los conductores, en cambio en el caso de referencia se obtienen mayores valores de campo magnético a mayores de distancias de la fuente.

En este caso se observa que con el cambio introducido en una de las ternas se logra mitigar en forma efectiva el campo magnético a grandes distancias, por ejemplo a $30 \mathrm{~m}$ el campo reduce su valor aproximadamente 4 veces. 


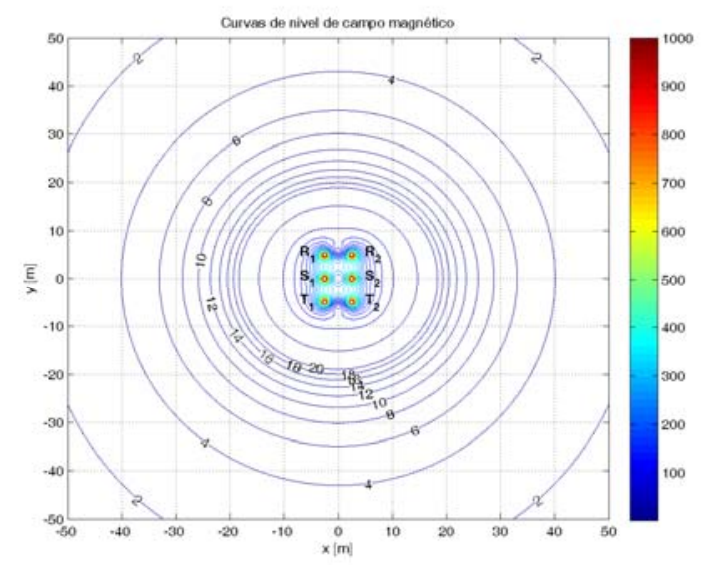

a) Configuración convencional

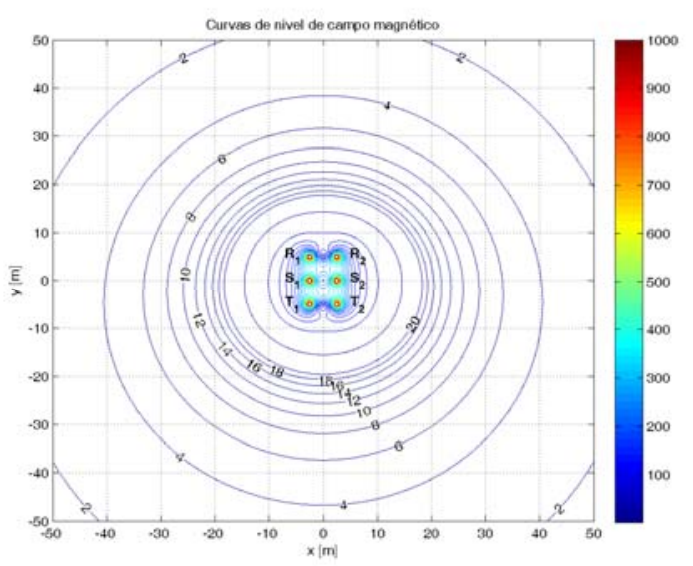

c) Configuración convencional

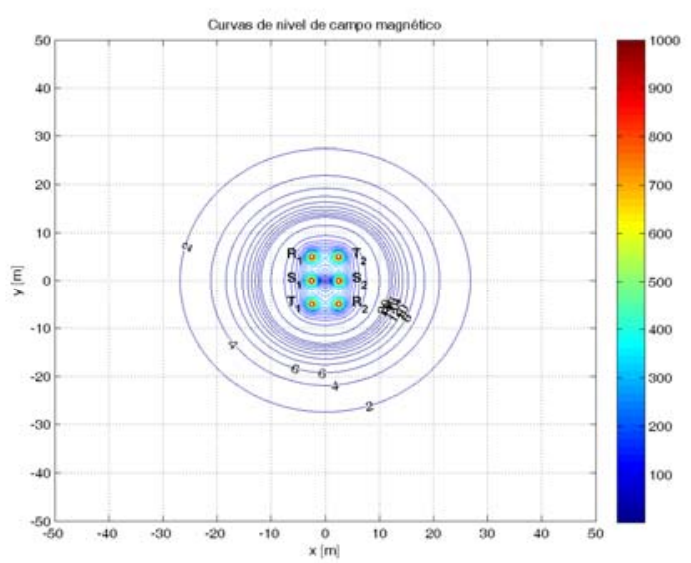

b) Configuración Traspuesta

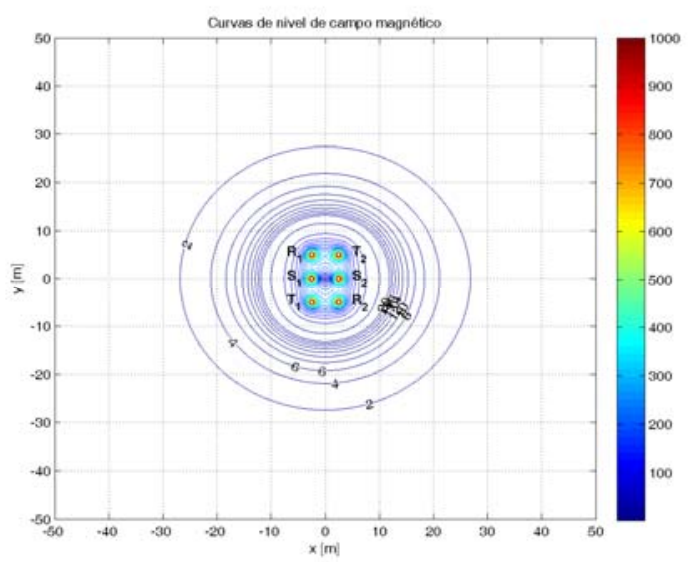

d) Configuración traspuesta

Fig. AVII 5 - Valores de campo B [ $\mu$ T], para circuitos trifásicos, I=2000 A, para doble terna.

Si se introduce un desbalance del $5 \%$ en una de las fases, en ambas ternas, se observa una leve distorsión en las curvas de nivel, pero los cambios en los valores en general no son significativos, esto puede apreciarse en las Fig. AVII 5 c) y d). En el sistema en el que se realizó la transposición puede observarse, comparando b) y d) que prácticamente no se encuentran cambios.

Si se tienen más de dos circuitos, puede estudiarse cual será la configuración óptima, que será la que arroje los valores más bajos de campo magnético en la zona de interés. 


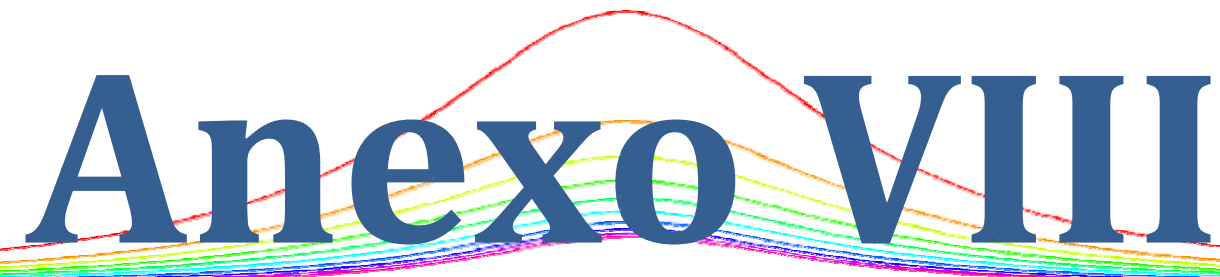

Condiciones de borde utilizadas en mitigación con placas metálicas 


\section{AVIII-1. Condiciones de borde utilizadas en mitigación con placas metálicas}

En los modelos tridimensionales implementados se utilizaron dos procedimientos de cálculo.

En primer lugar se realizaron cálculos utilizando el método de Biot-Savart. Se representan todas las fuentes de campo magnético como segmentos de corriente. Se define un área de interés y luego se realiza el cálculo de campo magnético en dicho área, en general el área se ubica a $1 \mathrm{~m}$ sobre el nivel del suelo, en el entorno de las fuentes de campo magnético que se quiere evaluar. En este caso no se imponen condiciones de borde, se aplica el principio de superposición, considerando el efecto de cada segmento de corriente.

Con este procedimiento se resolvieron los problemas presentados en:

\subsubsection{Campo Magnético generado por cables subterráneos simple terna}

\subsubsection{Campo Magnético generado por cables subterráneos con múltiples ternas}

5.4 Campo Magnético generado por Estaciones Transformadoras

5.5 Campo Magnético generado por Centros de Transformación.

En los casos en donde es necesario tener en cuenta el efecto de blindaje que proveen las corrientes inducidas en los elementos metálicos existentes, se debe recurrir a una formulación diferente del problema. Para ello se plantea el método de elementos finitos (MEF), generando una malla tridimensional, con elementos de diferentes tamaños, los elementos más pequeños se disponen en las zonas en donde se busca que el error sea menor. En estos casos el cálculo se realiza en un volumen y se imponen condiciones de borde en las caras exteriores del volumen, los resultados que se obtienen dependerán de estas condiciones.

En el AIV-1 se presento una descripción del software comercial (ELEKTRA SS) que se utilizó para los cálculos tridimensionales. En AIV-2 se indicaron las condiciones de bordes que admite el software, dichas condiciones se utilizan para aproximar el campo magnético a grandes distancias del problema (campo lejano, límites) y por otro lado permiten reducir el tamaño de la representación de elementos finitos de los problemas simétricos, esto se trato en AIV-3. Aquí se considera la aplicación de dichas condiciones al ejemplo presentado en el punto 6.4.2.

En la Fig. AVIII 1 se presenta una vista del modelo implementado en el ejemplo presentado en el punto 6.4.2, se consideran los conductores de las dos ternas en color rojo. En esta figura se aprecia la diferencia en el tamaño de los elementos, en donde se requiere mayor precisión los elementos son más pequeños. 


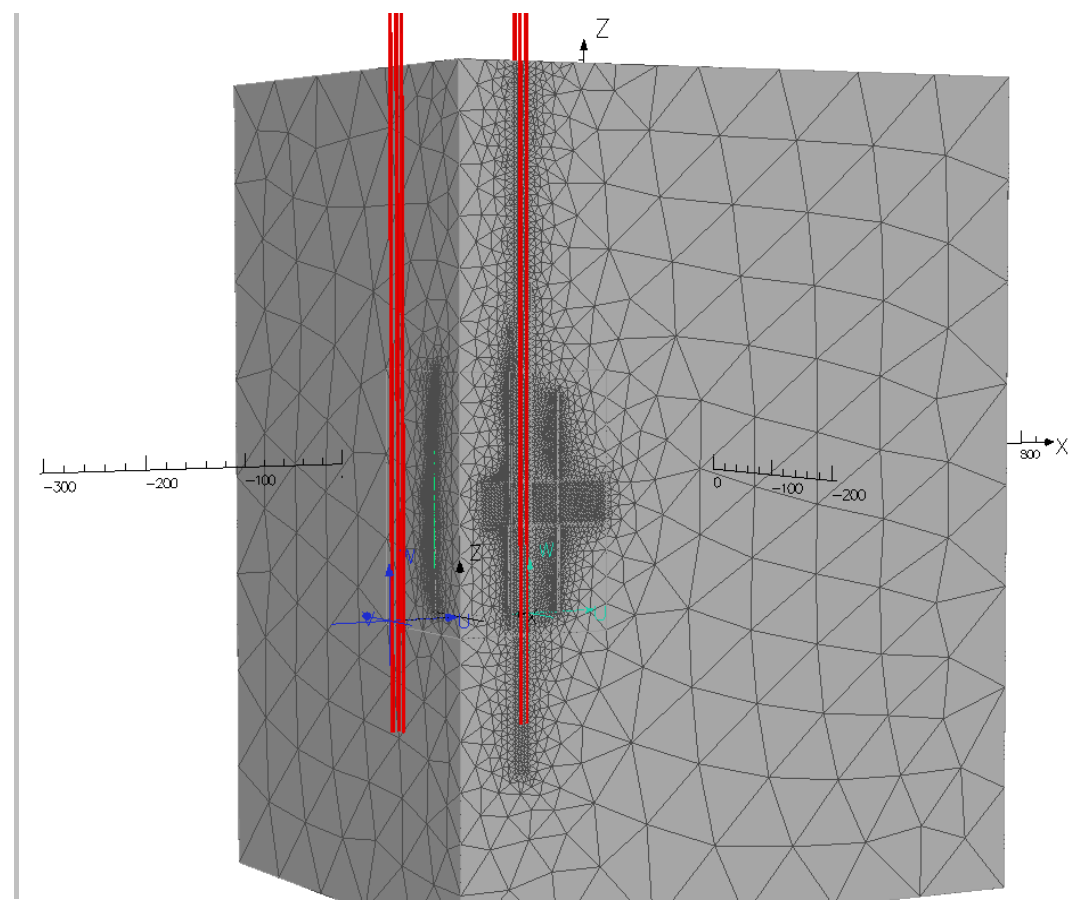

Fig. AVIII 1 - Modelo de elementos finitos, problema presentado en el 6.4.2.

Para el problema presentado en el punto 6.4.2 se utilizaron diferentes condiciones de borde.

A las caras exteriores del volumen de cálculo se les aplican condiciones de campo lejano, se definió al campo magnético como tangencial a las mismas. Esta misma condición de borde se utilizo para los planos perpendiculares a los conductores de fase. Se dimensionó el volumen de cálculo de forma tal que los bordes estén a una gran distancia de la zona de interés.

Por otro lado, dada la simetría que presenta el problema se redujo el modelo, resolviendo únicamente un cuarto del volumen original, con lo que resulta necesario aplicar condiciones de borde adicionales.

En la Fig. AVIII 2, se muestran imágenes con diferentes vistas del modelo de cálculo. En color anaranjado se destacan las caras exteriores, a las que se aplico condiciones de borde de campo lejano.
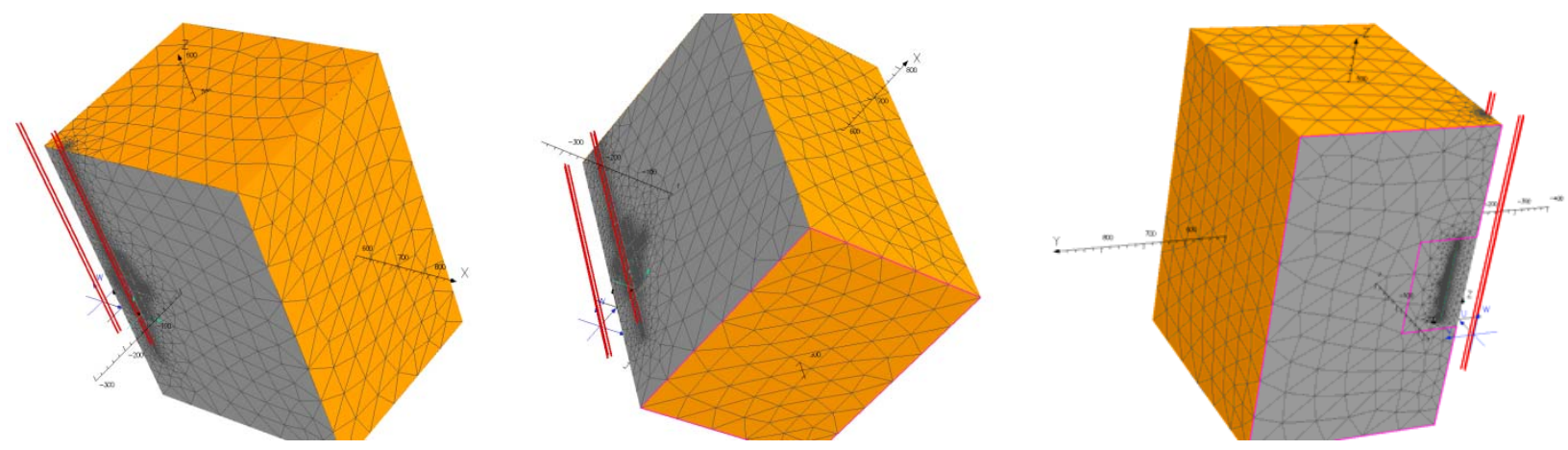

Fig. AVIII 2 - Modelo de elementos finitos, problema presentado en el punto 0, condiciones de borde de campo lejano, en las caras en color anaranjado.

En la Fig. AVIII 3 se presenta una vista en planta en donde se presenta la torre (Verde), las protecciones mecánicas (Azul) y los conductores de fase (Rojo) con la secuencia de 
energización. Como se mencionó el volumen de cálculo se redujo a 1/4 de su tamaño original, aprovechando la simetría que presenta el problema, con lo que se impusieron condiciones de bordes adicionales a las restantes caras de modelo, estas caras son internas al modelo. Las condiciones de borde aplicadas dependen de la simetría existente en las corrientes consideradas.

Los planos de simetría se eligieron de acuerdo al esquema presentado en la Fig. AVIII 3. Los planos se identifican como Reflejo en "X" y Reflejo en "Y". El eje " $z$ " es perpendicular al plano formado por los ejes " $x$ " e " $y$ " que se indican en la mencionada figura.

El plano identificado como Reflejo en "X", es un plano formado por los ejes " $\mathrm{x}$ " $\mathrm{y}$ " $\mathrm{z}$ ".

El plano identificado como Reflejo en "Y", es un plano formado por el eje " $y$ " y "z".

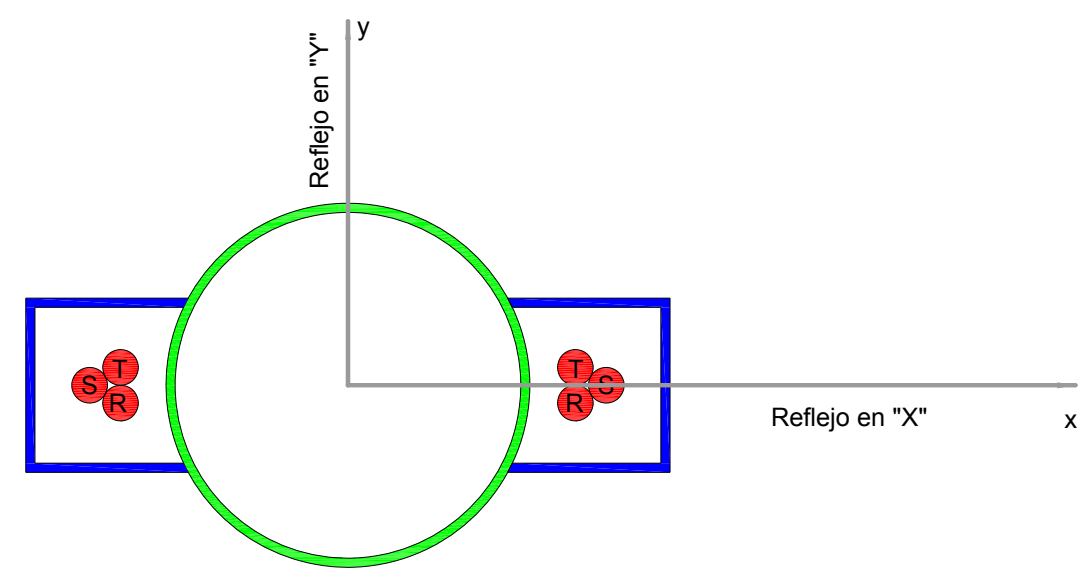

Fig. AVIII 3 - División del modelo para reducir la malla de elementos finitos, problema presentado en el punto 0 . Condiciones de borde para reducir el tamaño de la malla de elementos finitos.

Para definir e imponer las condiciones de borde a estas caras, es necesario evaluar cómo debe considerarse la distribución de corrientes.

Si se contempla que las corrientes de fase están desfasadas $120^{\circ}$ (Ir, Is e It), como se indica en la Fig. AVIII 4, es posible descomponer a las ternas de corrientes en sus partes real e imaginaria, esto permite dividir en dos las simulaciones. 


\section{$\mathrm{Ir} / 2=\mid \mathrm{t} / 2$}

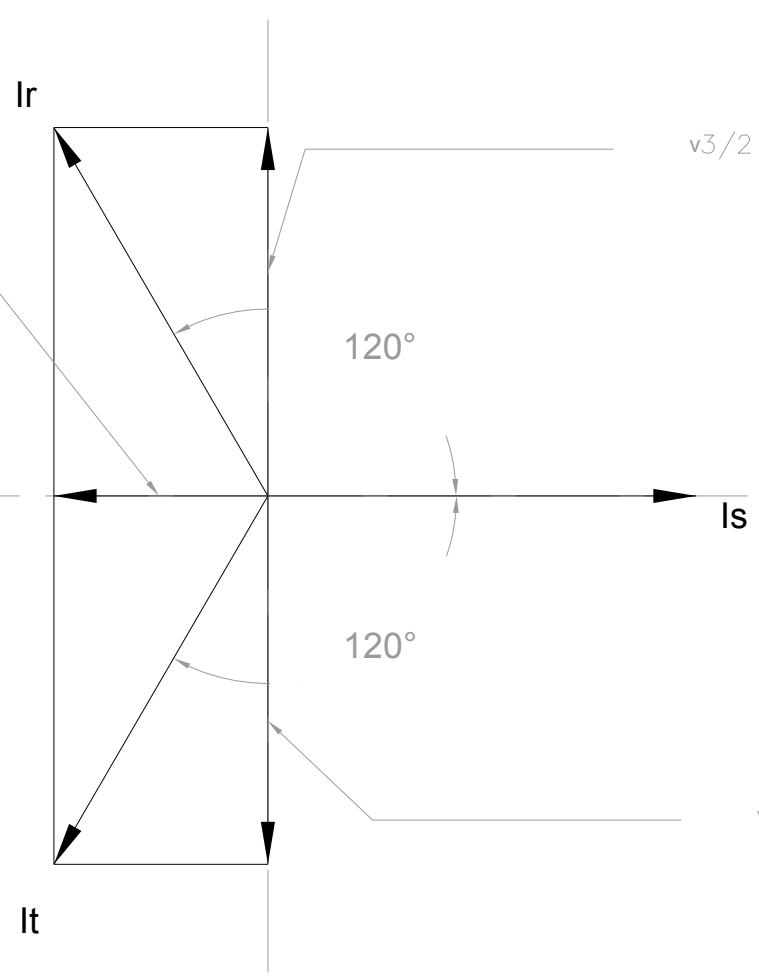

Fig. AVIII 4 - Descomposición de las ternas de corriente en parte real e imaginaria.

Si se considera la parte real, las ternas se conforman por corrientes $I_{s 1}=I s, I_{r 1}=I r / 2$ e $I_{t 1}=I t / 2$.

Si se considera la parte imaginaria las ternas se conforman por $I_{s 2}=0, I_{r 2}=(\sqrt{3} / 2)$ Ir e $I_{t 2}=$ $(\sqrt{3} / 2)$ It.

Con los resultados obtenidos en las dos simulaciones, se realizó la composición vectorial de los campos, considerando los resultados obtenidos en ambas.

En la Tabla AVIII 1 se presenta un resumen con los valores de corriente para las dos simulaciones. Además se indican las condiciones de borde consideradas en cada caso, para las caras internas.

Tabla AVIII 1- Condiciones de borde - Problema simétrico

\begin{tabular}{|l|l|l|l|}
\hline Simulación & \multicolumn{1}{|c|}{ Simulación } & 2 \\
\hline Corrientes & $\mathrm{Ir}^{*} 1 / 2 \mathrm{e}^{\mathrm{j} 180^{\circ}}$ & $\mathrm{Ir}_{2}=$ & $\mathrm{Ir}^{*} \sqrt{3} / 2 \mathrm{e}^{\mathrm{j} 90^{\circ}}$ \\
\hline $\mathrm{Ir}_{1}=$ & $\mathrm{Is} \mathrm{e}^{\mathrm{j} 0^{\circ}}$ & $\mathrm{Is}_{2}=$ & 0 \\
\hline $\mathrm{Is}_{1}=$ & $\mathrm{It}^{*} 1 / 2 \mathrm{e}^{\mathrm{j} 180^{\circ}}$ & $\mathrm{It}_{2}=$ & $\mathrm{It}^{*} \sqrt{3} / 2 \mathrm{e}^{-j 90^{\circ}}$ \\
\hline $\mathrm{It}_{1}=$ & \multicolumn{3}{|l}{} \\
\hline Condiciones de borde & Reflejo en “X" & B tangencial \\
\hline $\begin{array}{l}\text { Reflejo en } \\
\text { "X" }\end{array}$ & B normal \\
\hline $\begin{array}{l}\text { Reflejo en } \\
\text { "Y" }\end{array}$ & B normal & Reflejo en “Y” & B normal \\
\hline
\end{tabular}




\section{Anexo IX}

Configuraciones de lazos pasivos consideradas para CSAT Perfiles de campo magnético y FA obtenidos para el Caso 1 Perfiles de campo magnético y FA obtenidos para el Caso 2 Perfiles de campo magnético y FA obtenidos para el Caso 3 Perfiles de campo magnético y FA obtenidos para el Caso 4 Perfiles de campo magnético y FA obtenidos para el Caso 5 
AIX-1. Configuraciones de lazos pasivos consideradas para CSAT

Tabla XV - Configuraciones de lazos pasivos consideradas - Cables subterráneos.

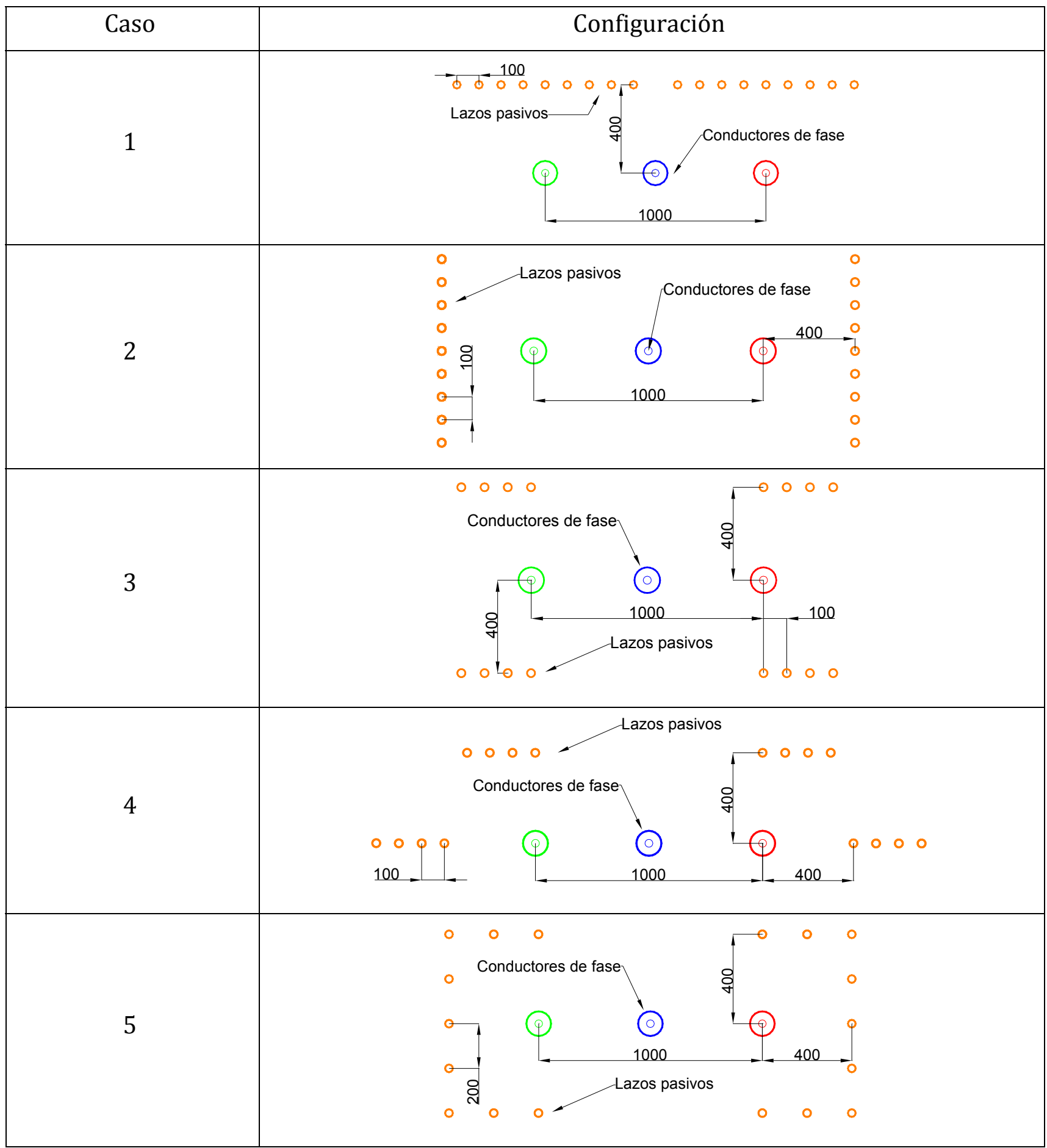




\section{AIX-2. $\quad$ Perfiles de campo magnético y FA obtenidos para el Caso 1}

En las Fig. AIX 1, Fig. AIX 3, Fig. AIX 5, Fig. AIX 7 y Fig. AIX 9 se presentan los perfiles obtenidos para las secciones mencionadas con conductores ubicados en un mismo plano, por encima de los conductores de fase. En las Fig. AIX 2, Fig. AIX 4, Fig. AIX 6, Fig. AIX 8 y Fig. AIX 10 se presentan los respectivos perfiles de FA.

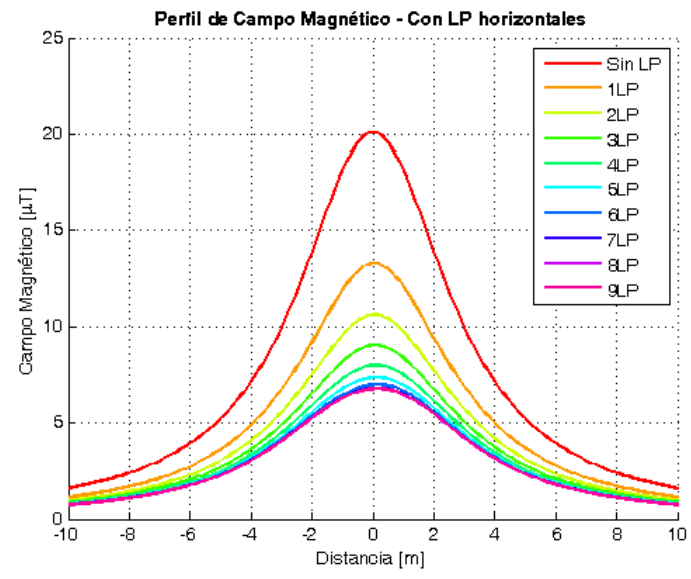

Fig. AIX 1 - Perfiles de campo magnético con lazos pasivos en un plano horizontal entre los conductores de fase y la área de interés, $\mathrm{I}=1000$ A, lazos de $240 \mathrm{~mm}^{2}$, Caso 1 - Tabla XV.

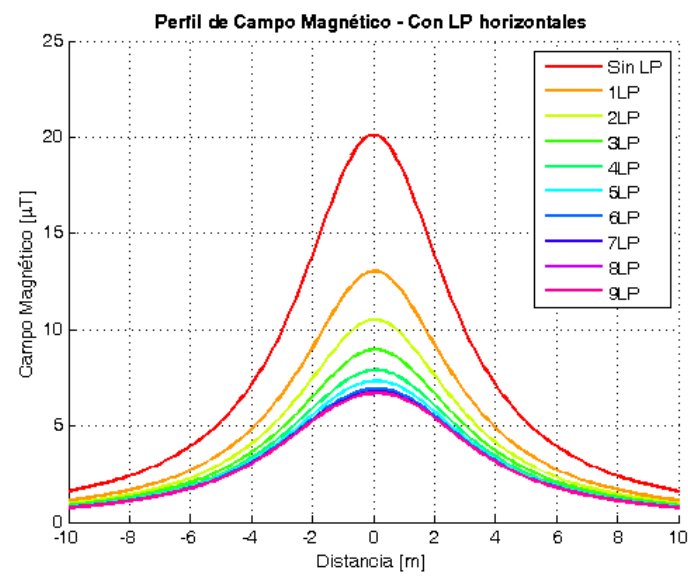

Fig. AIX 3 - Perfiles de campo magnético con lazos pasivos en un plano horizontal entre los conductores de fase y la área de interés, I= 1000 A, lazos de $300 \mathrm{~mm}^{2}$, Caso 1 - Tabla XV.

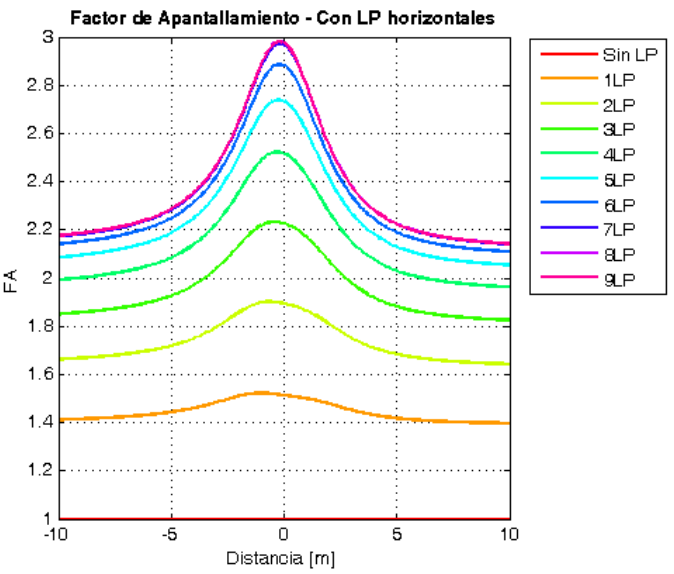

Fig. AIX 2 - Perfiles de FA con lazos pasivos en un plano horizontal entre los conductores de fase y la área de interés, $\mathrm{I}=1000 \mathrm{~A}$, lazos de $240 \mathrm{~mm}^{2}$, Caso 1 - Tabla XV.

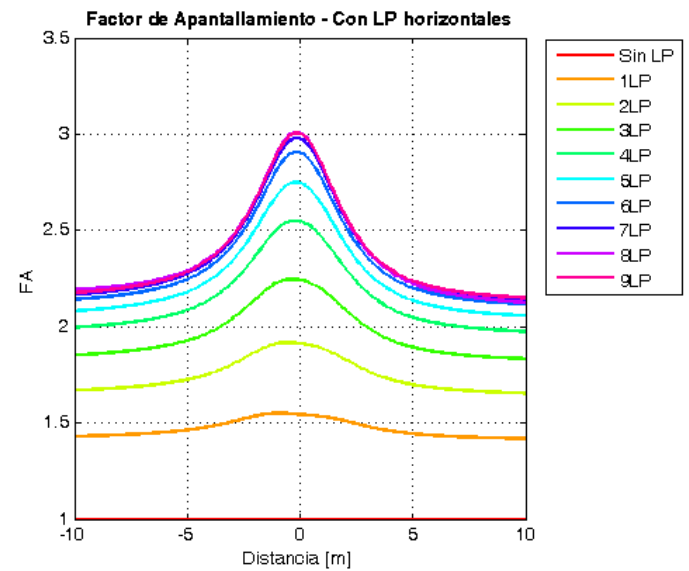

Fig. AIX 4 - Perfiles de FA con lazos pasivos en un plano horizontal entre los conductores de fase y la área de interés, I= $1000 \mathrm{~A}$, lazos de $300 \mathrm{~mm}^{2}$, Caso 1 - Tabla XV. 


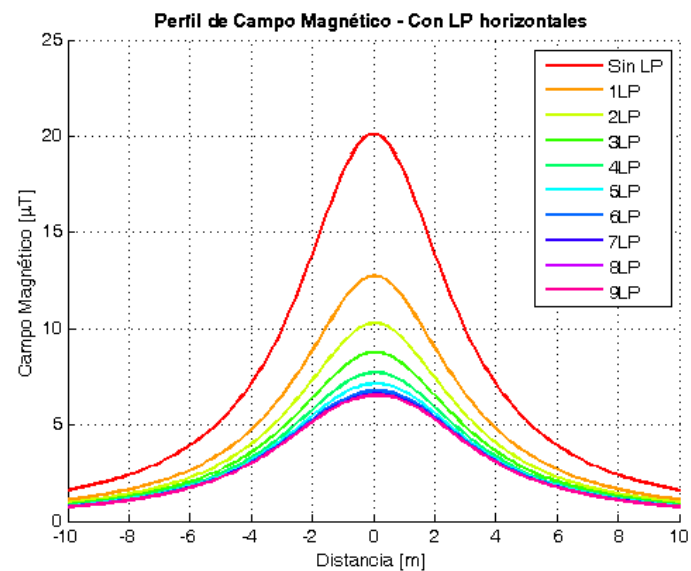

Fig. AIX 5 - Perfiles de campo magnético con lazos pasivos en un plano horizontal entre los conductores de fase y la área de interés, I= 1000 A, lazos de $400 \mathrm{~mm}^{2}$, Caso 1 - Tabla XV.

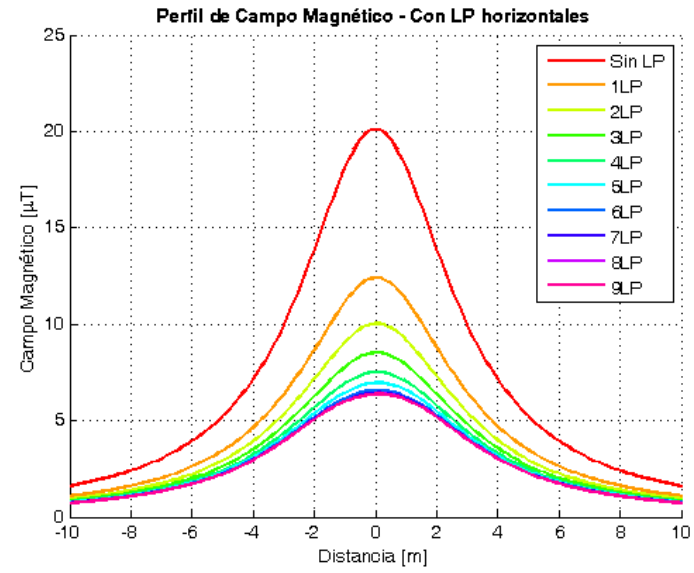

Fig. AIX 7 - Perfiles de campo magnético con lazos pasivos en un plano horizontal entre los conductores de fase y la área de interés, I= 1000 A, lazos de $500 \mathrm{~mm}^{2}$, Caso 1 - Tabla XV.

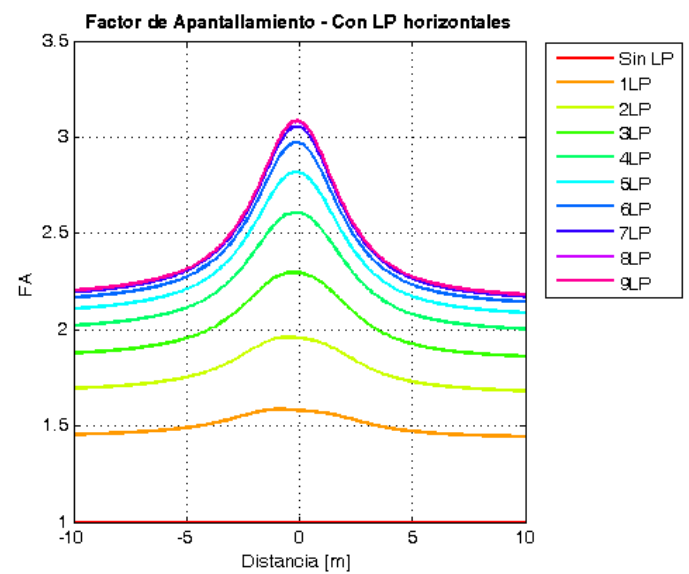

Fig. AIX 6 - Perfiles de FA con lazos pasivos en un plano horizontal entre los conductores de fase y la área de interés, $\mathrm{I}=1000 \mathrm{~A}$, lazos de $400 \mathrm{~mm}^{2}$, Caso 1 - Tabla XV.

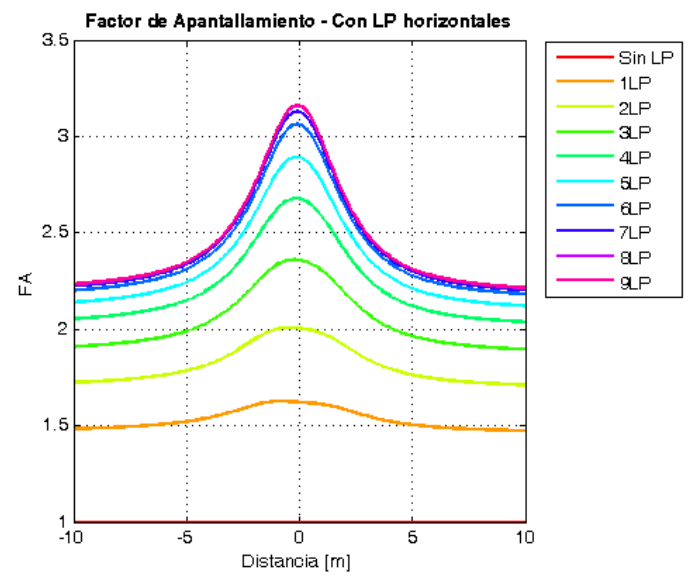

Fig. AIX 8 - Perfiles de FA con lazos pasivos en un plano horizontal entre los conductores de fase y la área de interés, I= $1000 \mathrm{~A}$, lazos de $500 \mathrm{~mm}^{2}$, Caso 1 - Tabla XV. 


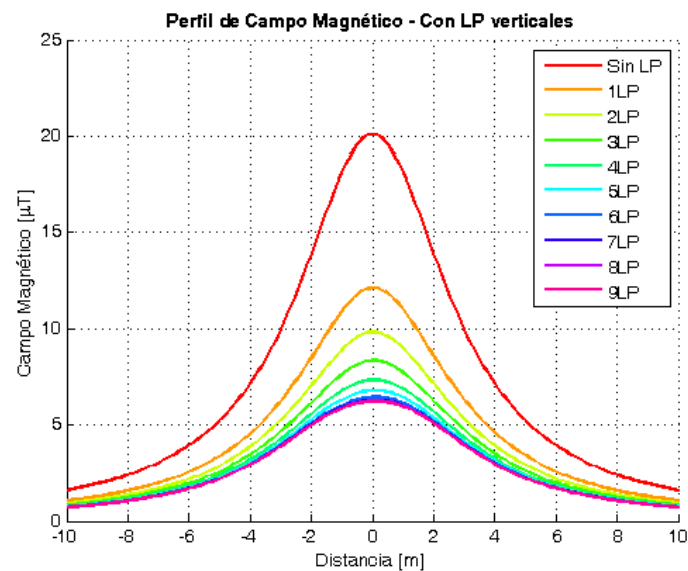

Fig. AIX 9- Perfiles de campo magnético con lazos pasivos en un plano horizontal entre los conductores de fase y la área de interés, $\mathrm{I}=1000$ A, lazos de $630 \mathrm{~mm}^{2}$, Caso 1 - Tabla XV.

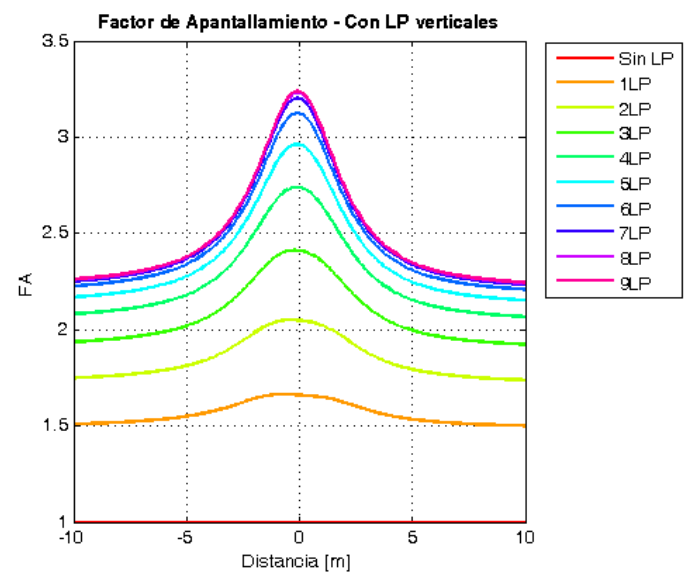

Fig. AIX 10- Perfiles de FA con lazos pasivos en un plano horizontal entre los conductores de fase y la área de interés, I= $1000 \mathrm{~A}$, lazos de $630 \mathrm{~mm}^{2}$, Caso 1 - Tabla XV.

\section{AIX-3. Perfiles de campo magnético y FA obtenidos para el Caso 2}

En las Fig. AIX 11, Fig. AIX 13, Fig. AIX 15, Fig. AIX 17 y Fig. AIX 19 se presentan los perfiles obtenidos para las secciones mencionadas, los conductores de fase se ubican en la zona central, los conductores de los lazos pasivos son ubicados en diferentes planos a ambos lados de los de fase, ver Caso 2 - Tabla XV. En las Fig. AIX 12, Fig. AIX 14, Fig. AIX 16, Fig. AIX 18 y Fig. AIX 20se presentan los respectivos perfiles de FA.

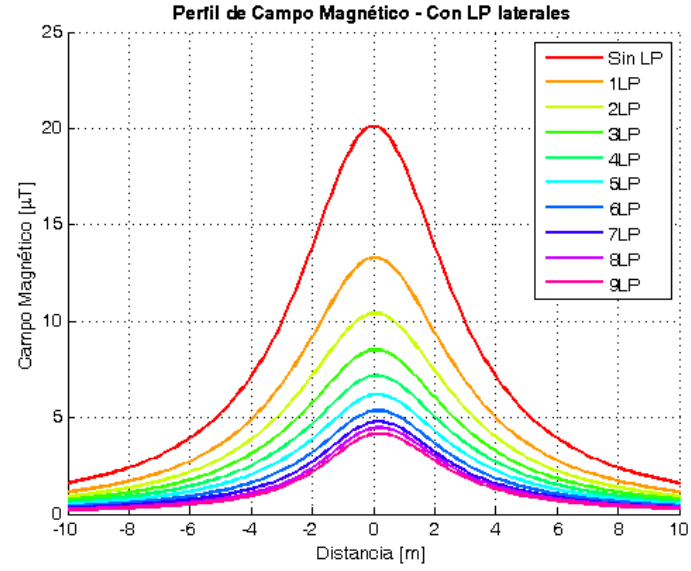

Fig. AIX 11 - Perfiles de campo magnético con lazos pasivos en diferentes planos a ambos lados de los conductores de fase, $\mathrm{I}=$ $1000 \mathrm{~A}$, lazos de $240 \mathrm{~mm}^{2}$, Caso 2 - Tabla XV.

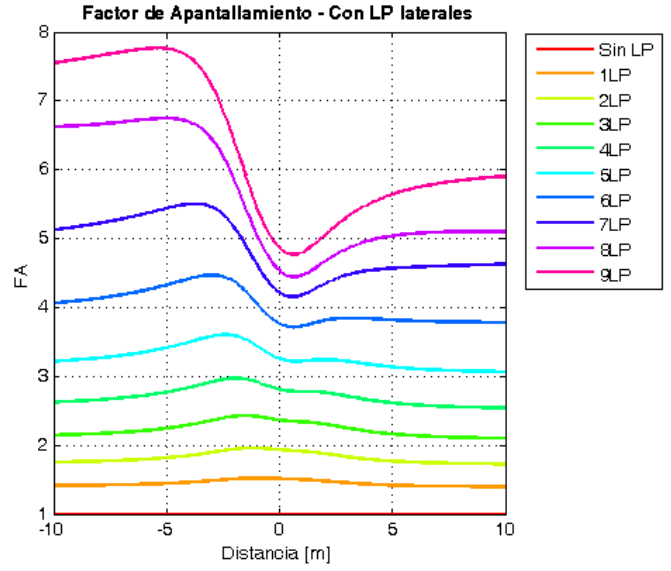

Fig. AIX 12 - Perfiles de FA con lazos pasivos en diferentes planos a ambos lados de los conductores de fase, $\mathrm{I}=1000 \mathrm{~A}$, lazos de $240 \mathrm{~mm}^{2}$, Caso 2 - Tabla XV. 


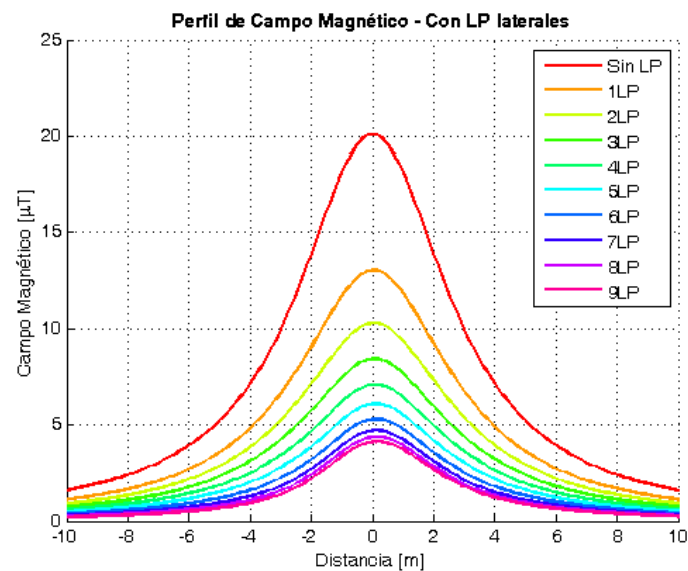

Fig. AIX 13 - Perfiles de campo magnético con lazos pasivos en diferentes planos a ambos lados de los conductores de fase, I= $1000 \mathrm{~A}$, lazos de $300 \mathrm{~mm}^{2}$, Caso 2 - Tabla XV.

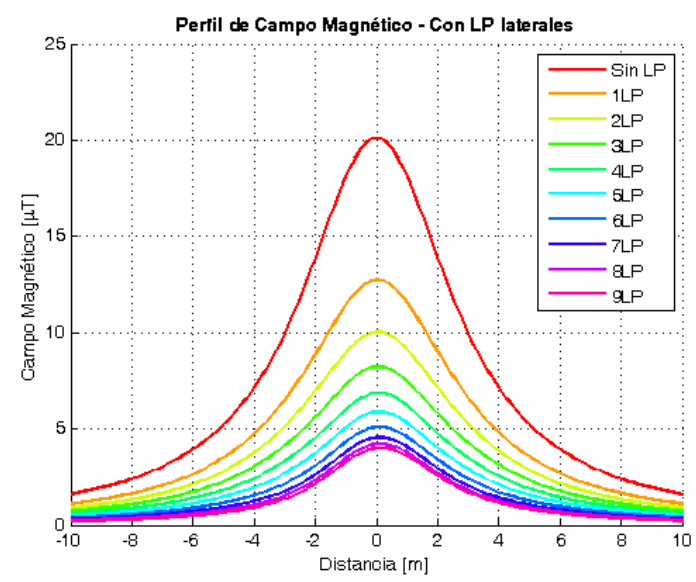

Fig. AIX 15 - Perfiles de campo magnético con lazos pasivos en diferentes planos a ambos lados de los conductores de fase, I= $1000 \mathrm{~A}$, lazos de $400 \mathrm{~mm}^{2}$, Caso 2 - Tabla XV.

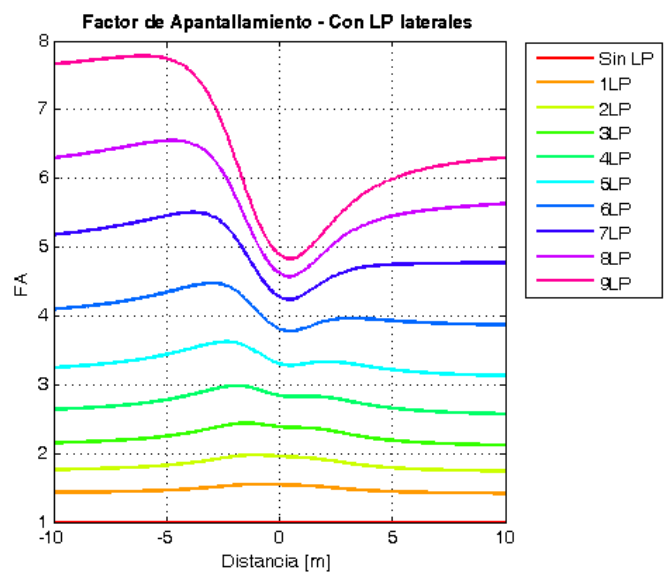

Fig. AIX 14 - Perfiles de FA con lazos pasivos en diferentes planos a ambos lados de los conductores de fase, $\mathrm{I}=1000 \mathrm{~A}$, lazos de $300 \mathrm{~mm}^{2}$, Caso 2 - Tabla XV.

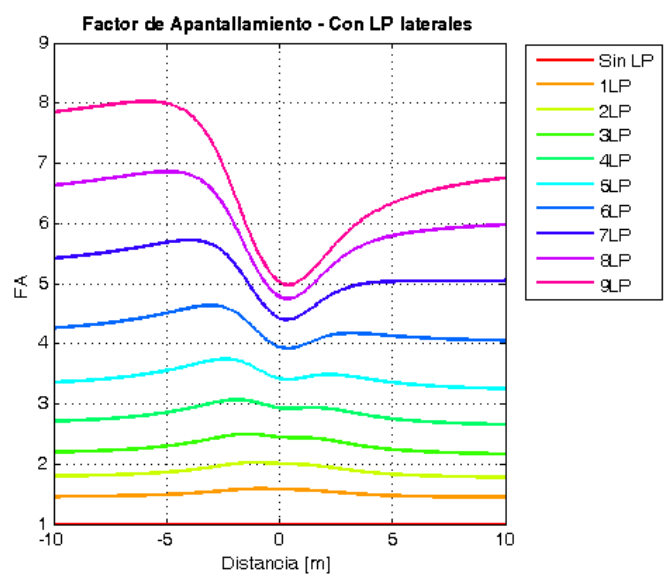

Fig. AIX 16 - Perfiles de FA con lazos pasivos en diferentes planos a ambos lados de los conductores de fase, $\mathrm{I}=1000 \mathrm{~A}$, lazos de $400 \mathrm{~mm}^{2}$, Caso 2 - Tabla XV. 


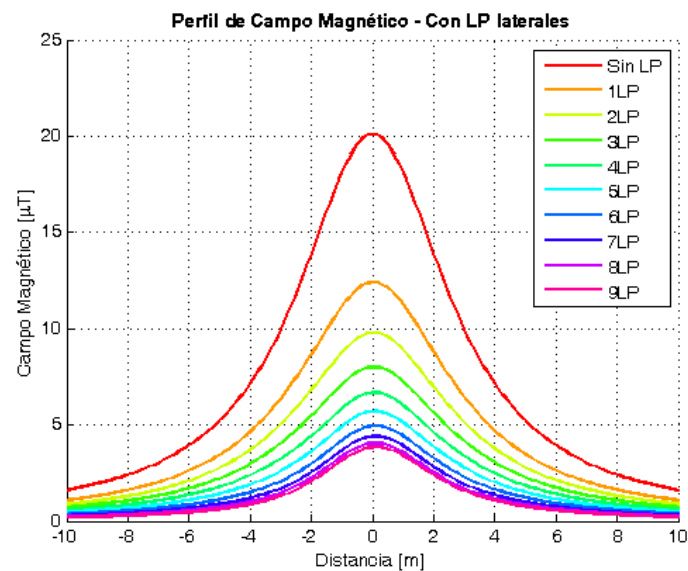

Fig. AIX 17 - Perfiles de campo magnético con lazos pasivos en diferentes planos a ambos lados de los conductores de fase, I= $1000 \mathrm{~A}$, lazos de $500 \mathrm{~mm}^{2}$, Caso 2 - Tabla XV.

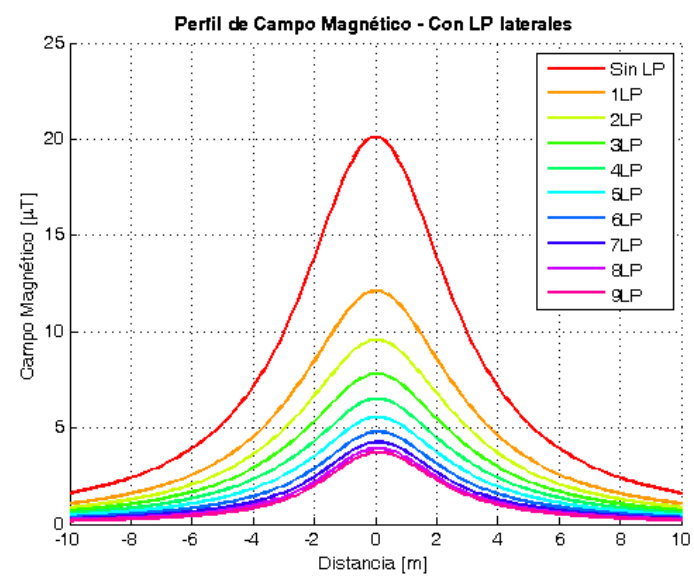

Fig. AIX 19 - Perfiles de campo magnético con lazos pasivos en diferentes planos a ambos lados de los conductores de fase, I= $1000 \mathrm{~A}$, lazos de $630 \mathrm{~mm}^{2}$, Caso 2 - Tabla XV.

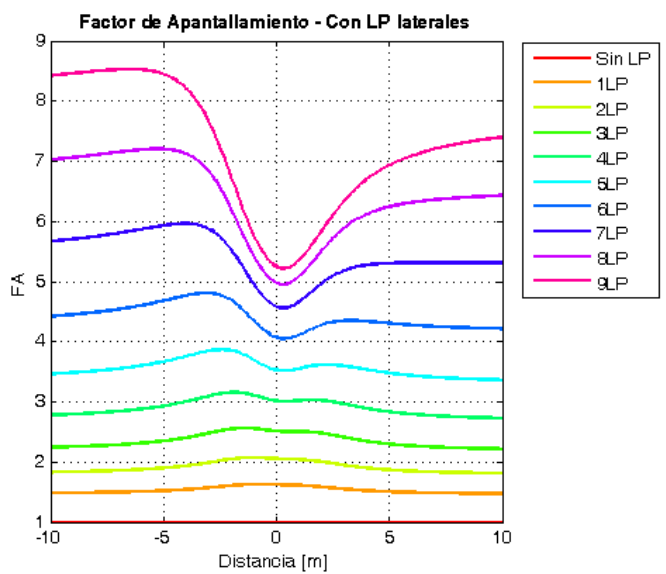

Fig. AIX 18 - Perfiles de FA con lazos pasivos en diferentes planos a ambos lados de los conductores de fase, $\mathrm{I}=1000 \mathrm{~A}$, lazos de $500 \mathrm{~mm}^{2}$, Caso 2 - Tabla XV.

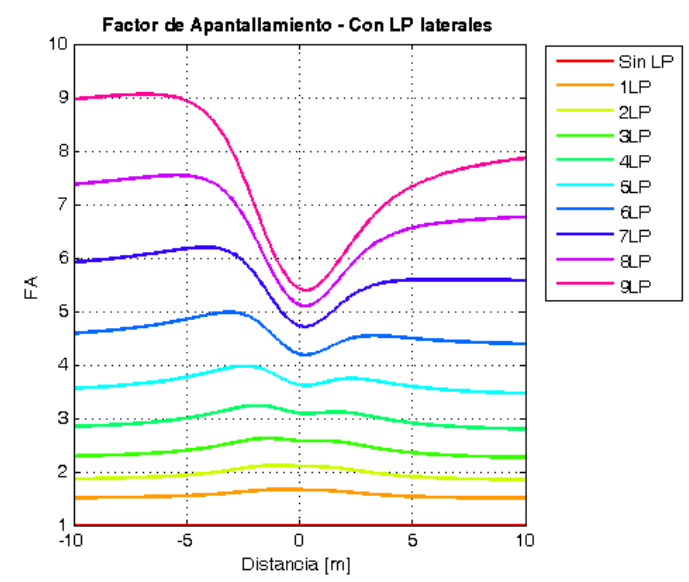

Fig. AIX 20 - Perfiles de FA con lazos pasivos en diferentes planos a ambos lados de los conductores de fase, $\mathrm{I}=1000 \mathrm{~A}$, lazos de $630 \mathrm{~mm}^{2}$, Caso 2 - Tabla XV. 


\section{AIX-4. $\quad$ Perfiles de campo magnético y FA obtenidos para el Caso 3}

En las Fig. AIX 21 y Fig. AIX 22 se presentan los perfiles de campo magnético y FA obtenidos para esta configuración con diferente número de conductores, obtenidos para el Caso 3 Tabla XV.

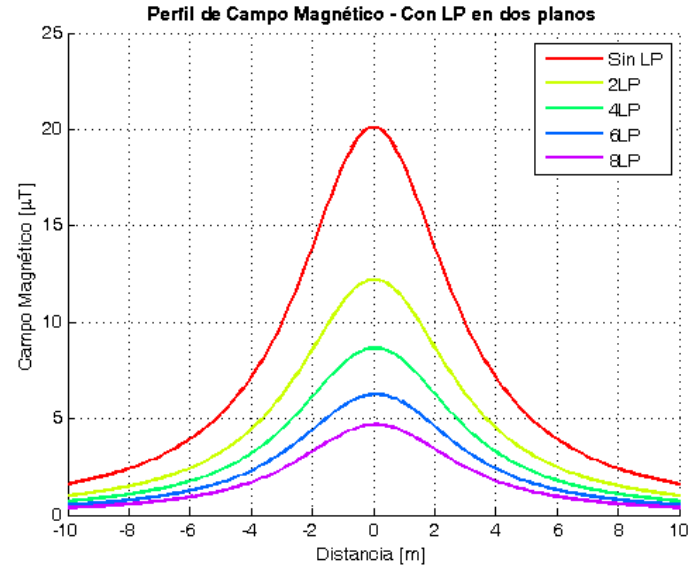

Fig. AIX 21 - Perfiles de campo magnético con lazos pasivos dos planos deferentes,

Caso 3 - Tabla XV, I= 1000 A, lazos de $630 \mathrm{~mm}^{2}$.

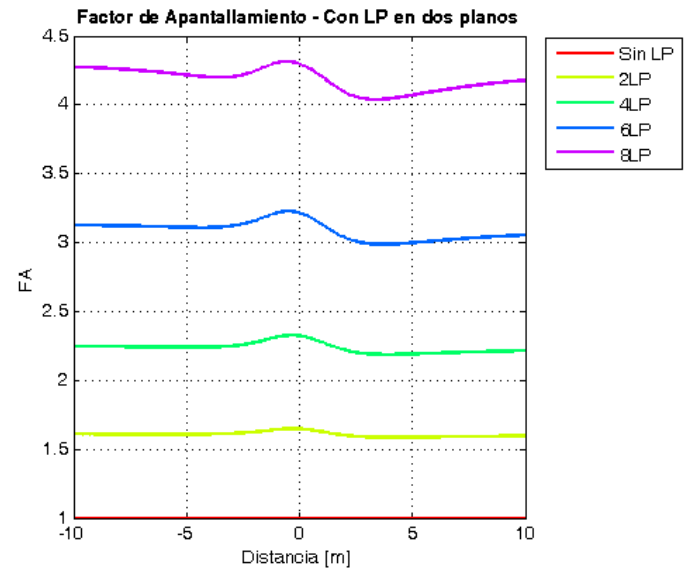

Fig. AIX 22 - Perfiles de FA con lazos pasivos dos planos deferentes, Caso 3 - Tabla XV, $\mathrm{I}=1000 \mathrm{~A}$, lazos de $630 \mathrm{~mm}^{2}$.

\section{AIX-5. $\quad$ Perfiles de campo magnético y FA obtenidos para el Caso 4}

En las Fig. AIX 23 y Fig. AIX 24 se presentan los perfiles de campo magnético y FA obtenidos para esta configuración con diferente número de conductores, obtenidos para el Caso 4 Tabla XV.

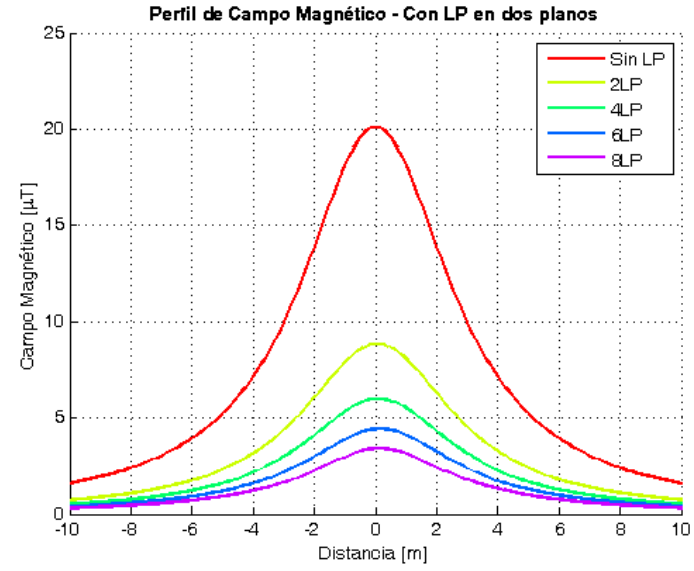

Fig. AIX 23 - Perfiles de campo magnético con lazos pasivos dos planos deferentes,

Caso 4 - Tabla XV, I= $1000 \mathrm{~A}$, lazos de $630 \mathrm{~mm}^{2}$

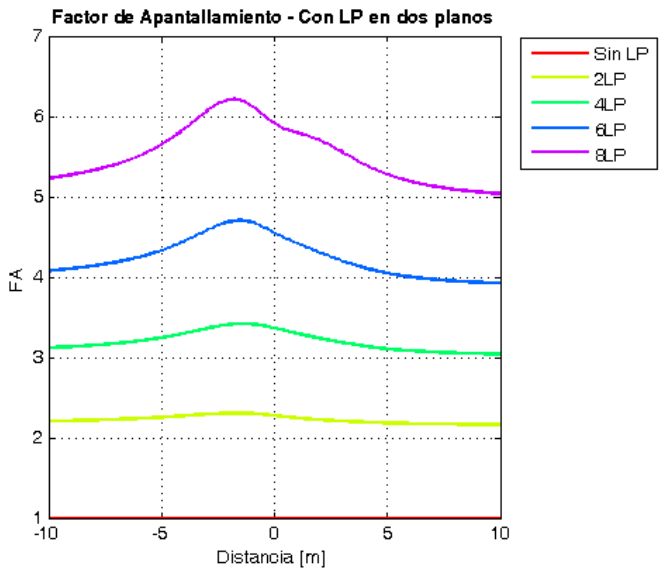

Fig. AIX 24 - Perfiles de FA con lazos pasivos dos planos deferentes, Caso 4 - Tabla XV, $\mathrm{I}=1000 \mathrm{~A}$, lazos de $630 \mathrm{~mm}^{2}$. 


\section{AIX-6. $\quad$ Perfiles de campo magnético y FA obtenidos para el Caso 5}

En las Fig. AIX 25 y Fig. AIX 26 se presentan los perfiles de campo magnético y FA obtenidos para esta configuración con diferente número de conductores, obtenidos para el Caso 5 Tabla XV.

Esta configuración es una combinación de lazos dispuestos en diferentes planos y lazos ubicados a ambos lados de los conductores de fase.

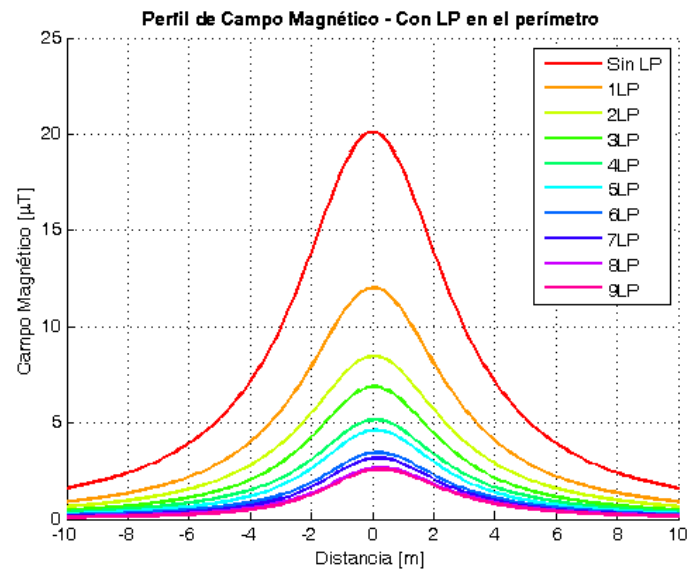

Fig. AIX 25 - Perfiles de campo magnético con lazos pasivos en el perímetro, Caso 5 Tabla XV, I= $1000 \mathrm{~A}$, lazos de $630 \mathrm{~mm}^{2}$.

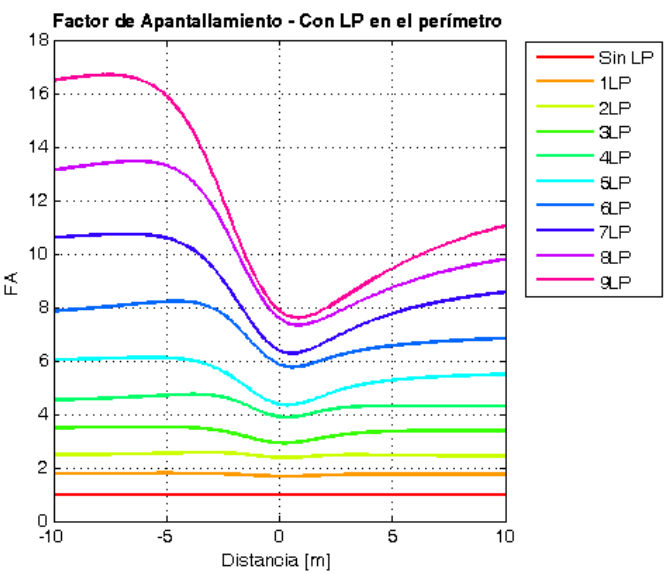

Fig. AIX 26 - Perfiles de FA con lazos pasivos en el perímetro, Caso 5 - Tabla XV, I= $1000 \mathrm{~A}$, lazos de $630 \mathrm{~mm}^{2}$. 